\title{
HOLOMORPHIC BUNDLES ON THE BLOWN-UP PLANE AND THE BAR CONSTRUCTION
}

\author{
JOÃO PAULO SANTOS
}

\begin{abstract}
We study the moduli space $\mathfrak{M}_{k}^{r}\left(\tilde{\mathbb{P}}_{q}^{2}\right)$ of rank $r$ holomorphic bundles with trivial determinant and second Chern class $c_{2}=k$, over the blowup $\tilde{\mathbb{P}}_{q}^{2}$ of the projective plane at $q$ points, trivialized on a rational curve. We show that, for $k=1,2$, we have a homotopy equivalence between $\mathfrak{M}_{k}^{r}\left(\tilde{\mathbb{P}}_{q}^{2}\right)$ and the degree $k$ component of the bar construction $\mathrm{B}\left(\mathfrak{M}^{r} \mathbb{P}^{2},\left(\mathfrak{M}^{r} \mathbb{P}^{2}\right)^{q},\left(\mathfrak{M}^{r} \tilde{\mathbb{P}}_{1}^{2}\right)^{q}\right)$. The space $\mathfrak{M}_{k}^{r}\left(\tilde{\mathbb{P}}_{q}^{2}\right)$ is isomorphic to the moduli space $\mathfrak{M I}_{k}^{r}\left(X_{q}\right)$ of charge $k$ based $S U(r)$ instantons on a connected sum $X_{q}$ of $q$ copies of $\overline{\mathbb{P}^{2}}$ and we show that, for $k=1,2$, we have a homotopy equivalence between $\mathfrak{M I}_{k}^{r}\left(X_{q} \# X_{s}\right)$ and the degree $k$ component of $\mathrm{B}\left(\mathfrak{M \mathcal { I } ^ { r }}\left(X_{q}\right), \mathfrak{M} \mathcal{I}^{r}\left(S^{4}\right), \mathfrak{M \mathcal { I } ^ { r }}\left(X_{s}\right)\right)$. Analogous results hold in the limit when $k \rightarrow \infty$. As an application we obtain upper bounds for the cokernel of the Atiyah-Jones map in homology, in the rank-stable limit.
\end{abstract}

\section{INTRODUCTION}

Let $\mathbb{P}^{2}=\mathbb{C}^{2} \cup \mathbb{P}^{1}$ be the projective plane seen as a compactification of $\mathbb{C}^{2}$ and denote by $L_{\infty} \subset \mathbb{P}^{2}$ the rational curve at infinity. Given a finite set $I \subset \mathbb{C}^{2}$, let $\tilde{\mathbb{P}}_{I}^{2}$ be the blowup of $\mathbb{P}^{2}$ along $I$. In this paper we study the moduli space $\mathfrak{M}_{I}^{r}$ of rank $r$ holomorphic bundles $\mathcal{E}$ on $\tilde{\mathbb{P}}_{I}^{2}$ with first Chern class $c_{1}(\mathcal{E})=0$, trivialized at $L_{\infty}$. These bundles are topologically classified by their second Chern class $c_{2}=k$ and by Bogolomov's inequality [29, Theorem 4.1] we must have $k \geq 0$. We write $\mathfrak{M}_{I}^{r}=\coprod_{k \geq 0} \mathfrak{M}_{I, k}^{r}$ where $\mathfrak{M}_{I, k}^{r}$ denotes the subspace of bundles with $c_{2}=k$. By collapsing $L_{\infty} \subset \tilde{\mathbb{P}}_{I}^{2}$ to a point we obtain a smooth 4-manifold diffeomorphic to a connected sum of \#I copies of the projective plane with reversed orientation, which we represent by $\#_{I} \overline{\mathbb{P}^{2}}$. It was shown in [10] and [32] that $\mathfrak{M}_{I, k}^{r}$ is isomorphic as a real analytic space to the moduli space $\mathfrak{M I}_{k}^{r}\left(\#_{I} \overline{\mathbb{P}^{2}}\right)$ of instantons with charge $k$ on a $S U(r)$ bundle over $\#_{I} \overline{\mathbb{P}^{2}}$ (a standard reference for instantons is [16]).

It has long been known [46, 47, 15] that, given 4-manifolds $X$ and $Y$, under rather general hypothesis one can glue together instantons on $X$ and $Y$ to obtain an instanton on the connected sum $X \# Y$. When $X=Y=S^{4}$, this construction gives the moduli space $\mathfrak{M I}^{r}\left(S^{4}\right)$ the structure of a graded homotopy algebra over the $C_{4}$ operad 7, section 6], where $C_{4}$ denotes the little 4-cubes operad [33, section 4]. In particular, $\mathfrak{M} \mathcal{I}^{r}\left(S^{4}\right)$ behaves homologically like an algebra over the $C_{4}$ operad. For general $X$ and $Y$ we have a map $\mathfrak{M I}_{k_{1}}^{r}(X) \times \mathfrak{M I}_{k_{2}}^{r}(Y) \rightarrow \mathfrak{M I}_{k_{1}+k_{2}}^{r}(X \# Y)$. Conversely, by "stretching the neck" an instanton $\nabla_{A}$ on $X \# Y$ decomposes as an instanton on $X$, an instanton on $Y$ and several instantons (the number depends on $\nabla_{A}$ ) on infinite cylinders [36, Theorem 6.3.3], which we may regard as an element in the space $\mathfrak{M I}^{r}(X) \times \mathfrak{M} \mathcal{I}^{r}\left(S^{4}\right)^{n} \times \mathfrak{M} \mathcal{I}^{r}(Y)$ for some $n$. These spaces are the building

2010 Mathematics Subject Classification. 14D21, 58D27, 14J60, 55P48. 
blocks of the bar construction $\mathrm{B}\left(\mathfrak{M I}^{r}(X), \mathfrak{M I}^{r}\left(S^{4}\right), \mathfrak{M} \mathcal{I}^{r}(Y)\right.$ ) (see section 2.1.3 below), which suggests that this bar construction should be related to the moduli space $\mathfrak{M I}^{r}(X \# Y)$. In this paper we investigate this relationship.

We will state our results in terms of the moduli space $\mathfrak{M}_{I}^{r}$ of holomorphic bundles on $\tilde{\mathbb{P}}_{I}^{2}$. Holomorphic bundles on a blowup $\tilde{X}$ of a complex surface $X$ can be studied using gluing techniques (see [11, 41, [20]). For $k=2$, a neighbourhood of the subspace of bundles over $\tilde{X}$ which are non-trivial on one of the exceptional divisors can be obtained by gluing in "concentrated" framed holomorphic bundles on $\mathbb{P}^{2}$ 42, Propositions 4.8, 4.9], mimicking the description of a neighbourhood of points at infinity in the compactified moduli space of instantons (see [16, section 8.2]). The bar construction is homeomorphic to the nerve of the open cover thus obtained.

The procedure described above for $k=2$ can also be implemented, for any $k$, in the limit when the rank $r$ goes to infinity: the gluing maps correspond to Whitney sum. Whitney sum is not strictly associative so some care has to be taken in defining the bar construction. Our approach is similar to the one in [1]: we replace the simplicial category with a topological category whose spaces of morphisms are homotopy equivalent to the discrete spaces of morphisms in the simplicial category.

In finite rank we define the gluing maps for $k=1,2$ using the monad description of holomorphic bundles introduced in [14], 26]. Although the same techniques could in principle be used for $k>2$, there are several complicating factors. For $k=2$ the only non-trivial products we need to consider are of the form $\mathfrak{M}_{J, 1}^{r} \times \mathfrak{M}_{K, 1}^{r} \rightarrow$ $\mathfrak{M}_{J \cup K, 2}^{r}$ and we can further reduce to the case where $\# J, \# K \leq 1$ where we have simple monad descriptions of the moduli spaces. Also, the gluing maps depend on a choice of disjoint open sets in $\mathbb{C}^{2}$ (of the form $U \times \mathbb{C}$ ). Since there is never a need to multiply together more than 2 non-trivial factors, associativity is not an issue so we don't need the little cubes operad. All these simplifying factors fail for $k>2$.

1.1. Results. For $k=1,2$ we obtain a description of the moduli space $\mathfrak{M}_{I}^{r}$ in terms of the moduli spaces $\mathfrak{M}_{\emptyset}^{r}$ and $\mathfrak{M}_{x}^{r}$, with $x \in I$ : using results in [42, we construct, for any disjoint finite sets $I_{1}, \ldots, I_{n} \subset \mathbb{C}^{2}$, maps $\boxplus: \mathfrak{M}_{I_{1}, k_{1}}^{r} \times \cdots \times \mathfrak{M}_{I_{n}, k_{n}}^{r} \rightarrow \mathfrak{M}_{I, k}^{r}$, where $I=\bigcup_{i} I_{i}$ and $k=\sum_{i} k_{i} \leq 2$. Using these maps we build the degree 0,1 and 2 components of the bar constructions $\mathrm{B}\left(\mathfrak{M}_{I}^{r}, \mathfrak{M}_{\emptyset}^{r}, \mathfrak{M}_{J}^{r}\right)$ and $\mathrm{B}\left(\mathfrak{M}_{\emptyset}^{r}, \prod_{x \in I} \mathfrak{M}_{\emptyset}^{r}, \prod_{x \in I} \mathfrak{M}_{x}^{r}\right)$,
which we represent by $\mathrm{B}(\cdots)_{\leq 2}$.

Theorem 1.1. Let $I=\left\{x_{1}, \ldots, x_{q}\right\} \subset \mathbb{C}^{2}$. Then:

(1) The map $\boxplus: \mathfrak{M}_{\emptyset}^{r} \times\left(\prod_{i} \mathfrak{M}_{x_{i}}^{r}\right) \rightarrow \mathfrak{M}_{I}^{r}$ induces a map

$$
h_{\boxplus}: \mathrm{B}\left(\mathfrak{M}_{\emptyset}^{r}, \prod_{i} \mathfrak{M}_{\emptyset}^{r}, \prod_{i} \mathfrak{M}_{x_{i}}^{r}\right)_{\leq 2} \rightarrow \mathfrak{M}_{I, \leq 2}^{r}
$$

which is a homotopy equivalence.

(2) If $I=J \cup K$, with $J \cap K=\emptyset$, then the map $\boxplus: \mathfrak{M}_{J}^{r} \times \mathfrak{M}_{K}^{r} \rightarrow \mathfrak{M}_{I}^{r}$ induces a map $\mathrm{B}\left(\mathfrak{M}_{J}^{r}, \mathfrak{M}_{\emptyset}^{r}, \mathfrak{M}_{K}^{r}\right)_{\leq 2} \rightarrow \mathfrak{M}_{I, \leq 2}^{r}$ which is a homotopy equivalence.

Direct sum with a trivial rank $r^{\prime}-r$ bundle induces a map $\mathfrak{M}_{I}^{r} \rightarrow \mathfrak{M}_{I}^{r^{\prime}}$ and we let $\mathfrak{M}_{I}^{\infty}=\operatorname{colim} \mathfrak{M}_{I}^{r}$. In [28, Theorem 1.2], 40, Theorem 3], 8, Theorem 1.1] it was shown that we have homotopy equivalences $\mathfrak{M}_{\emptyset}^{\infty} \simeq \coprod_{k \geq 0} B U(k)$ and $\mathfrak{M}_{x}^{\infty} \simeq \coprod_{k \geq 0} B U(k) \times B U(k)$ (for $x \in \mathbb{C}^{2}$ ). For $J \subset I$, pullback of bundles induces a map $\pi_{J, I}^{*}: \mathfrak{M}_{J}^{r} \rightarrow \mathfrak{M}_{I}^{r}$. Combining the maps $\pi_{J, I}^{*}$ with Whitney sum allows us to define, for each $I \subset \mathbb{C}^{2}$, a bar construction (see sections 4 and 5), which we denote 
by:

$$
\left\|\mathfrak{B}_{I}\right\|=\mathrm{B}\left(\mathfrak{M}_{\emptyset}^{\infty}, \prod_{x \in I} \mathfrak{M}_{\emptyset}^{\infty}, \prod_{x \in I} \mathfrak{M}_{x}^{\infty}\right)
$$

and a map:

$$
h_{I}:\left\|\mathfrak{B}_{I}\right\| \rightarrow \mathfrak{M}_{I}^{\infty}
$$

The second Chern class of the bundles gives a grading of the spaces $\left\|\mathfrak{B}_{I}\right\|$. In the limit when $r \rightarrow \infty$ the maps $\boxplus$ agree with the Whitney sum maps so Theorem 1.1 implies that $h_{I}$ is a homotopy equivalence in degrees 1 and 2 (see Theorem 8.47).

Conjecture 1. The map $h_{I}:\left\|\mathfrak{B}_{I}\right\| \rightarrow \mathfrak{M}_{I}^{\infty}$ is a homotopy equivalence.

For disjoint finite sets $I$ and $J$ we have (see Proposition 5.12):

$$
\mathrm{B}\left(\left\|\mathfrak{B}_{I}\right\|, \mathfrak{M}_{\emptyset}^{\infty},\left\|\mathfrak{B}_{J}\right\|\right) \simeq\left\|\mathfrak{B}_{I \cup J}\right\| .
$$

Assuming Conjecture 1 holds, it follows that $\mathfrak{M}_{I \cup J}^{\infty} \simeq \mathrm{B}\left(\mathfrak{M}_{I}^{\infty}, \mathfrak{M}_{\emptyset}^{\infty}, \mathfrak{M}_{J}^{\infty}\right)$. Also, from a finite rank version of equation (1.2) (see Proposition 8.42) it can be seen that parts (1) and (2) of Theorem 1.1 are equivalent.

We also show that, in order to check the conjecture in a given degree $k$, it is enough to show that the map $h_{I}$ is a homotopy equivalence when $\# I \leq k$ :

Theorem 1.3. If, for every finite set $J \subset I$ with $\# J \leq k$, the map $h_{J}$ is a homotopy equivalence in degree $k$, then $h_{I}$ is a homotopy equivalence in degree $k$.

Note that finite rank versions of Theorem 1.3 and equation (1.2) (see equation (8.46) and Proposition 8.42) imply Theorem 1.1 for $k=1$.

There is an analog to Theorem 1.1 in the limit when $k \rightarrow+\infty$. Write $\mathfrak{M} \mathcal{I}_{I}^{r}=$ $\mathfrak{M \mathcal { I } ^ { r }}\left(\#_{I} \overline{\mathbb{P}^{2}}\right)$ and let $\mathscr{C}_{I, k}^{r}$ denote the space of all connections on an $S U(r)$ bundle over $\#_{I} \overline{\mathbb{P}^{2}}$ with second Chern class equal to $k \bmod$ gauge equivalence. This space is homotopically equivalent to $\operatorname{Map}_{*}\left(\#_{I} \overline{\mathbb{P}^{2}}, B S U(r)\right)$ [13, Theorem 1.3]. For each degree $k \geq 0$ we have $\mathfrak{M I}_{I, k}^{r} \subset \mathscr{C}_{I, k}^{r}$ and in [4], Taubes described, for $k^{\prime}>k$, homotopy equivalences $\mathscr{C}_{I, k}^{r} \rightarrow \mathscr{C}_{I, k^{\prime}}^{r}$ which restrict to maps $\mathfrak{M I}_{I, k}^{r} \rightarrow \mathfrak{M} \mathcal{I}_{I, k^{\prime}}^{r}$, and showed that "in the direct limit" the natural inclusion maps

$$
\imath_{k}: \mathfrak{M I} \mathcal{I}_{I, k}^{r} \rightarrow \mathscr{C}_{I, k}^{r}
$$

induce a homotopy equivalence $\mathfrak{M I}_{I, \infty}^{r} \stackrel{\simeq}{\rightarrow} \mathscr{C}_{I, \infty}^{r}$. The $r=+\infty$ case of the following theorem shows that Conjecture 1 holds in the limit when $k \rightarrow+\infty$ :

Theorem 1.5. Let $r$ be either a non-negative integer or $r=+\infty$. Then there is a homotopy equivalence:

$$
\mathrm{B}\left(\mathscr{C}_{\emptyset, \infty}^{r}, \prod_{x \in I} \mathscr{C}_{\emptyset, \infty}^{r}, \prod_{x \in I} \mathscr{C}_{x, \infty}^{r}\right) \rightarrow \mathscr{C}_{I, \infty}^{r}
$$

As an application, we study the image in homology of the map $\imath_{k}$ in equation (1.4) in the limit when $r \rightarrow+\infty$. This map was first studied by Atiyah and Jones in [2] in the finite rank case. They conjectured that $\imath_{k}$ induces an isomorphism in homology and homotopy through a range $q(k, r)$ that grows with $k$. The original conjecture (over $S^{4}$ ) was proven in [6, 28, 49] and generalizations were proved in [24, 20]. We compute the homology of $\left\|\mathfrak{B}_{I}\right\|$ and show that the map $\left\|\mathfrak{B}_{I, k}\right\| \rightarrow \mathscr{C}_{I, k}^{\infty}$ is an isomorphism in homology up to homological dimension $2 k+1$. As a corollary we show that: 
Theorem 1.6. Let $h_{I, k}:\left\|\mathfrak{B}_{I, k}\right\| \rightarrow \mathfrak{M}_{I, k}^{\infty}$ be the $c_{2}=k$ component of the map $h_{I}$. Then $h_{I, k}$ is injective in homology and, in homological degree up to $2 k+1$, we have:

(1) The inclusion map $\imath_{k}: \mathfrak{M} \mathcal{I}_{I, k}^{\infty} \rightarrow \mathscr{C}_{I, k}^{\infty}$ induces surjective homomorphisms in homology.

(2) The map $h_{I, k}$ is an isomorphism in homology up to the range of validity of the Atiyah-Jones conjecture.

We also give upper bounds for the cokernel of the map $\imath_{k}$ in homology up to dimension $4 k-2 \# I+7$; furthermore, since the space $\left\|\mathfrak{B}_{I, k}\right\|$ is simply connected (Proposition 10.8), statements analogous to (1) and (2) above also hold in homotopy (see Theorem [1.6] ${ }^{*}$ on page 60).

The paper is organized as follows: In section 2 we briefly recall the definitions and basic facts about the bar construction, homotopy colimits and operads. In section 3, we describe the moduli spaces of holomorphic bundles $\mathfrak{M}_{I}^{r}$ and, in the limit when $r \rightarrow \infty$, give them the structure of $E_{\infty}$-spaces. In sections 4 and 5 , we describe the bar constructions in the limit when $r \rightarrow \infty$. In section 6. we prove Theorem [1.3. In section 7 , we define the products $\boxplus$ used in the statement of Theorem 1.1 and prove their properties. In section 8 , we prove Theorem 1.1 and use it to show that $h_{I, k}$ is a homotopy equivalence for $k=1,2$. In section 9, we prove Theorem 1.5. In section 10, we compute the homology of $\left\|\mathfrak{B}_{I}\right\|$ and prove Theorem 1.6. In appendix A we gather some technical proofs concerning the bar construction.

\section{Preliminaries on operads and the bar construction}

The two-sided bar construction for monoids and its generalization for algebras over operads play a central role in our work. We gather here some terminology and facts which we'll be using throughout the paper. Let Top be the category of compactly generated spaces in the sense of [50] and let hTop be the homotopy category. Unless otherwise stated, mapping spaces always have the compact open topology.

2.1. The bar construction. In this section we provide some background on the bar construction and homotopy colimits. More details can be found in 23].

2.1.1. Diagrams of spaces. A topological category $\mathfrak{C}$ is a small category with topologized morphism sets such that composition is continuous and ob $\mathfrak{C} \subset$ mor $\mathfrak{C}$ is a closed cofibration. A continuous functor $\mathfrak{C}_{1} \rightarrow \mathfrak{C}_{2}$ is said to be an equivalence of categories if it is the identity on objects and a homotopy equivalence on the morphism spaces.

A $\mathfrak{C}$-diagram of spaces is a continuous functor $X: \mathfrak{C} \rightarrow$ Top. A weak equivalence between $\mathfrak{C}$-diagrams $X_{1}, X_{2}$ is a natural transformation $\overline{\alpha:} X_{1} \rightarrow X_{2}$ such that $\alpha(A): X_{1}(A) \rightarrow X_{2}(A)$ is a homotopy equivalence for all $A \in$ ob $\mathfrak{C}$. We say $\alpha$ is a homeomorphism if $\alpha(A)$ is a homeomorphism for all $A \in$ ob $\mathfrak{C}$. A functor between topological categories $i: \mathfrak{C}_{1} \rightarrow \mathfrak{C}_{2}$ induces a pullback of diagrams: given a $\mathfrak{C}_{2}$-diagram $X: \mathfrak{C}_{2} \rightarrow$ Top we let $i^{*} X=X \circ i: \mathfrak{C}_{1} \rightarrow$ Top.

Example 2.1. Let $\mathcal{U}=\left\{U_{\alpha}\right\}_{\alpha \in A}$ be an open covering of a space $X$ and for each finite subset $\sigma \subset A$ let $U_{\sigma}=\bigcap_{\alpha \in \sigma} U_{\alpha}$. Let $\mathfrak{C}_{\mathcal{U}}$ be the category whose objects are the non-empty open sets $U_{\sigma}$ and whose morphisms are the inclusions. We refer to the tautological diagram of spaces $N: \mathfrak{C}_{\mathcal{U}} \rightarrow \underline{\text { Top }}$ as the nerve of the open cover $\mathcal{U}$. 
2.1.2. Simplicial spaces. Let $\Delta$ be the simplicial category, whose objects are the finite ordered sets $[n]=\{0, \ldots, n\}$ and whose morphisms $\Delta(m, n)$ are the order preserving maps $\mu:[m] \rightarrow[n]$. Let $\Delta^{\mathrm{op}}$ be the opposite category. The $\Delta^{\mathrm{op}}$-diagrams of spaces are called simplicial spaces. Given a simplicial space $X_{\bullet}: \Delta^{\mathrm{op}} \rightarrow$ Top, its geometric realization [33, Definition 11.1] is the topological space $\left|X_{\bullet}\right|={\overline{\left(\coprod_{n}\right.}}_{0_{0}} \Delta^{n} \times$ $\left.X_{n}\right) / \sim$, where $\Delta^{n}$ is the standard $n$-simplex and the equivalence relation is generated by $(\xi \cdot \mu, a) \sim(\xi, \mu \cdot a)$ for every $\xi \in \Delta^{m}, a \in X_{n}$ and $\mu \in \Delta(m, n)$. A natural transformation $X_{\bullet} \rightarrow Y_{\bullet}$ induces a map $\left|X_{\bullet}\right| \rightarrow\left|Y_{\bullet}\right|$.

Example 2.2. A space $Y$ can be identified with the simplicial space $Y_{\bullet}$ with $Y_{n}=Y$ for all $[n] \in$ ob $\Delta$ : we have a canonical homeomorphism $\left|Y_{\bullet}\right| \cong Y$ [33, Lemma 11.8]. Given a simplicial space $X_{\bullet}$, a sequence of maps

$$
f_{n}: X_{n} \rightarrow Y \quad \text { with } \quad f_{m}(\mu x)=f_{n}(x) \quad\left(x \in X_{n}, \mu \in \Delta(m, n)\right)
$$

induces a map $\left|X_{\bullet}\right| \rightarrow Y$.

2.1.3. The bar construction for monoids. Let $G$ be a topological monoid (that is, $G$ has an associative product with unit) acting on the left and on the right on topological spaces $Y$ and $X$ respectively. The bar construction $\mathrm{B}(X, G, Y)$ is the homotopy invariant version of the orbit space $X \times_{G} Y$. It is defined as the geometric realization of the simplicial space $\mathrm{B} \cdot(X, G, Y)$ defined on objects by

$$
\mathrm{B}_{n}(X, G, Y)=X \times G^{n} \times Y
$$

and defined on morphisms as follows: Given $\mu \in \Delta(m, n)$ let $\mu_{\alpha}=\mu(\alpha)$, with $\alpha=1, \ldots, m$; then we can write

$$
X \times G^{n} \times Y=\left(X \times G^{\mu_{0}}\right) \times\left(\prod_{\alpha=1}^{m} G^{\mu_{\alpha}-\mu_{\alpha-1}}\right) \times\left(G^{n-\mu_{m}} \times Y\right)
$$

and the map $X \times G^{n} \times Y \rightarrow X \times G^{m} \times Y$ is induced by the maps

$$
X \times G^{\mu_{0}} \rightarrow X, \quad G^{n-\mu_{m}} \times Y \rightarrow Y \quad \text { and } \quad G^{\mu_{\alpha}-\mu_{\alpha-1}} \rightarrow G \quad(\alpha=1, \ldots, m) .
$$

defined by the product in $G$ and the actions of $G$ on $X$ and $Y$.

If we let $G^{\text {op }}$ denote the space $G$ with the opposite monoid structure, then $G^{\text {op }}$ acts on the left on $X$ and on the right on $Y$ and $\mathrm{B}\left(Y, G^{\mathrm{op}}, X\right)$ is naturally homeomorphic to $\mathrm{B}(X, G, Y)$.

Proposition 2.4. The actions $G \times Y \rightarrow Y$ and $X \times G \rightarrow X$ induce maps $\mathrm{B}(G, G, Y) \rightarrow Y$ and $\mathrm{B}(X, G, G) \rightarrow X$ respectively, which are homotopy equivalences.

Proof. For the map B $(G, G, Y) \rightarrow Y$ see [23, Proposition 3.1(5)] (taking into account Remark 2.5 below). The result for the map $\mathrm{B}(X, G, G) \rightarrow X$ then follows from the homeomorphism $\mathrm{B}(X, G, G) \cong \mathrm{B}\left(G^{\mathrm{op}}, G^{\mathrm{op}}, X\right)$.

One important example is the case where $X=Y=*$ and $G$ is a group. Then $B G=\mathrm{B}(*, G, *)$ is the classifying space of $G$ and $E G=\mathrm{B}(G, G, *) \simeq *$ is the universal principal bundle over $B G$. 
2.1.4. The categorical bar construction. Given a topological category $\mathfrak{C}$, a $\mathfrak{C}$-diagram $Y$ and a $\mathfrak{C}^{\text {op }}$-diagram $X$, the bar construction $\mathrm{B}(X, \mathfrak{C}, Y)$ is the geometrical realization of the simplicial space defined on objects by

$$
\begin{aligned}
& \mathrm{B}_{n}(X, \mathfrak{C}, Y) \\
= & \underset{A, B \in \mathfrak{C}}{\amalg}\left\{\left(x ; f_{n}, \ldots, f_{1} ; y\right): f_{n} \circ \cdots \circ f_{1} \in \mathfrak{C}(A, B) \text { is defined, } y \in Y(A), x \in X(B)\right\}
\end{aligned}
$$

and defined on morphisms by composition of arrows in $\mathfrak{C}$ and by the evaluation maps $\mathfrak{C}(A, B) \times Y(A) \rightarrow Y(B)$ and $X(B) \times \mathfrak{C}(A, B) \rightarrow X(A)$.

Remark 2.5. The bar construction for monoids in section 2.1.3 is the special case when the category $\mathfrak{C}$ has only one object; then its space of morphisms is a monoid $G$ and a $\mathfrak{C}$-diagram is a space $Y$ with a left action of $G$.

2.1.5. Homotopy colimit. Let $X$ be a $\mathfrak{C}^{2}$-diagram. The colimit of $X$ is $\operatorname{colim}_{\mathfrak{C}} X=$ $\left(\coprod_{a \in \text { ob } \mathfrak{C}} X_{a}\right) / \sim$ where the equivalence relation identifies $x \sim \mu \cdot x$ for all $x \in X_{a}$ and $\mu \in \mathfrak{C}(a, b)$. The homotopy colimit is the homotopy invariant version of the colimit.

Definition 2.6. Let $\mathfrak{C}$ be a topological category, let $X$ be a $\mathfrak{C}$-diagram and let $*$ be the constant $\mathfrak{C}^{\text {op }}$-diagram on a one-point space. The homotopy colimit of $X$ along $\mathfrak{C}$ is

$$
\underset{\mathfrak{C}}{\operatorname{hcolim}} X=\mathrm{B}(*, \mathfrak{C}, X) \text {. }
$$

For convenience, we state here some basic properties of the homotopy colimit which we will be using later:

\section{Proposition 2.7.}

(1) Given a simplicial space $X$, there is a simplicial space $\tau X$ weakly equivalent to $X$ whose geometric realization $|\tau X|$ is homotopically equivalent to hcolim $_{\Delta}$ op $X$. Furthermore, if $X$ is a good simplicial space (in the sense of 44, Definition A.4]) then hcolim ${ }_{\Delta \text { op }} X \simeq|X| \simeq|\tau X|$.

(2) Given a $\mathfrak{C}$-diagram $X$ and a topological space $Y$, we have hcolim $(Y \times X) \cong$ $Y \times \operatorname{hcolim} X$.

(3) If $X_{1}, \ldots, X_{n}$ are $\mathfrak{C}$-diagrams then hcolim $\mathfrak{C}\left(\coprod_{i} X_{i}\right)=\coprod_{i}\left(\right.$ hcolim $\left._{\mathfrak{C}} X_{i}\right)$.

Proof. Statement (1) is [44, Propositions A.1(iv) and A.3], and statements (2) and (3) follow easily from the definition and properties of the bar construction [23, Proposition 3.1].

Given a functor $F: \mathfrak{C} \rightarrow \mathfrak{D}$ and an object $D \in \mathfrak{D}$, the undercategory $D \downarrow F$ is the category whose objects are the pairs $(f, C)$ with $C \in \mathfrak{C}$ and $f: D \rightarrow F(C)$ and whose morphisms $p:(f, C) \rightarrow\left(f^{\prime}, C^{\prime}\right)$ are the morphisms $p: C \rightarrow C^{\prime}$ in $\mathfrak{C}$ such that $F(p) \circ f=f^{\prime}$. The overcategory $F \downarrow D$ is the category whose objects are the pairs $(C, f)$ with $f: F(C) \rightarrow D$ and whose morphisms $p:(C, f) \rightarrow\left(C^{\prime}, f^{\prime}\right)$ are the morphisms $p: C \rightarrow C^{\prime}$ in $\mathfrak{C}$ such that $f^{\prime} \circ F(p)=f$. We say the functor $F$ is right cofinal if:

$$
B(*, D \downarrow F, *) \cong * \text { for all } D \in \mathfrak{D} .
$$

If $F$ is right cofinal then the induced map hcolim $\mathfrak{C}^{*} X \rightarrow$ hcolim $_{\mathfrak{D}} X$ is a homotopy equivalence [23, Proposition 4.4]. 
2.2. Operads. We wish to generalize the bar construction of section2.1.3 to spaces $A$ which, instead of a strictly associative product, have a family of products with each product associative only up to homotopy. The technical tool to handle this situation is the theory of operads, of which we give some background in this section. General treatments of this topic can be found in [31, 18.

Before we define operad we need to introduce some notation about symmetric groups:

Definition 2.9. Let $\Sigma_{n}$ denote the symmetric group of permutations of $n$ elements.

- Given an $n$-tuple $\left(a_{1}, \ldots, a_{n}\right)$ and $\sigma \in \Sigma_{n}$ we write

$$
\sigma\left(a_{1}, \ldots, a_{n}\right)=\left(a_{\sigma^{-1}(1)}, \ldots, a_{\sigma^{-1}(n)}\right) .
$$

- Let $j_{1}, \ldots, j_{k}$ be non-negative integers, let $j=j_{1}+\cdots+j_{k}$ and consider the partition $P$ of $\{1, \ldots, j\}$ into $k$ intervals of sizes $j_{1}, \ldots, j_{k}$.

- Given $\sigma \in \Sigma_{k}$, let $\sigma_{j_{1}, \ldots, j_{k}} \in \Sigma_{j}$ be the block permutation which permutes the $k$ blocks of the partition $P$ in the same way that $\sigma$ permutes the elements of $\{1, \ldots, k\}$.

- Given $\tau_{i} \in \Sigma_{j_{i}}$ let $\tau_{1} \oplus \cdots \oplus \tau_{k} \in \Sigma_{j}$ be the image of $\left(\tau_{1}, \ldots, \tau_{k}\right)$ under the inclusion $\Sigma_{j_{1}} \times \cdots \times \Sigma_{j_{k}} \rightarrow \Sigma_{j}$ induced by the partition $P$.

An operad $\mathcal{P}$ is a sequence of spaces $\mathcal{P}(n)$ parametrizing $n$-adic operations $A^{n} \rightarrow$ $A$ on a space $A$. Note that, given maps $\theta: A^{k} \rightarrow A$ and $\theta_{i}: A^{j_{i}} \rightarrow A$, composition defines a map $\theta \circ\left(\theta_{1}, \ldots, \theta_{k}\right): A^{j_{1}+\cdots+j_{k}} \rightarrow A$.

Definition 2.10. An operad $\mathcal{P}$ (in the category Top) is a sequence of spaces $\{\mathcal{P}(n)\}_{n \geq 0}$ with $\mathcal{P}(0)=\{*\}$, together with the following data: a right action of the symmetric group $\Sigma_{n}$ on $\mathcal{P}(n)$; a unit $\mathbf{1} \in \mathcal{P}(1)$; and continuous maps

$$
\gamma: \mathcal{P}(k) \times \mathcal{P}\left(j_{1}\right) \times \cdots \times \mathcal{P}\left(j_{k}\right) \rightarrow \mathcal{P}(j) \quad\left(j=j_{1}+\cdots+j_{k}\right)
$$

which we also represent by $\gamma\left(\theta ; \theta_{1}, \ldots, \theta_{k}\right)=\theta \circ\left(\theta_{1}, \ldots, \theta_{k}\right)$, satisfying:

Equivariance: Given $\theta \in \mathcal{P}(k), \theta_{i} \in \mathcal{P}\left(j_{i}\right), \sigma \in \Sigma_{k}$ and $\tau_{i} \in \Sigma_{j_{i}}$ we have

$$
\begin{aligned}
\theta \circ\left(\theta_{1} \tau_{1}, \ldots, \theta_{k} \tau_{k}\right) & =\left(\theta \circ\left(\theta_{1}, \ldots, \theta_{k}\right)\right)\left(\tau_{1} \oplus \cdots \oplus \tau_{k}\right) ; \\
(\theta \sigma) \circ\left(\theta_{1}, \ldots, \theta_{k}\right) & =\left(\theta \circ \sigma\left(\theta_{1}, \ldots, \theta_{k}\right)\right) \sigma_{j_{1}, \ldots, j_{k}} .
\end{aligned}
$$

\section{Associativity:}

$$
\begin{aligned}
\theta \circ\left(\theta_{1} \circ\left(\theta_{11}, \ldots, \theta_{1 j_{1}}\right), \ldots,\right. & \left.\theta_{k} \circ\left(\theta_{k 1}, \ldots, \theta_{k j_{k}}\right)\right) \\
& =\left(\theta \circ\left(\theta_{1}, \ldots, \theta_{k}\right)\right) \circ\left(\theta_{11}, \ldots, \theta_{1 j_{1}}, \ldots, \theta_{k 1}, \ldots, \theta_{k j_{k}}\right) .
\end{aligned}
$$

Unit: $\mathbf{1} \circ \theta=\theta \circ(\mathbf{1}, \ldots, \mathbf{1})=\theta$.

A morphism of operads $\mathcal{P}_{1} \rightarrow \mathcal{P}_{2}$ is a sequence of equivariant maps $\mathcal{P}_{1}(n) \rightarrow \mathcal{P}_{2}(n)$ such that the induced map $\psi: \coprod_{n} \mathcal{P}_{1}(n) \rightarrow \coprod_{n} \mathcal{P}_{2}(n)$ satisfies $\psi(\mathbf{1})=\mathbf{1}$ and

$$
\psi\left(\theta \circ\left(\theta_{1}, \ldots, \theta_{k}\right)\right)=(\psi \theta) \circ\left(\psi \theta_{1}, \ldots, \psi \theta_{k}\right) .
$$

Example 2.11. Given a based topological space $A$, the sequence $\operatorname{End}_{A}(n)=\operatorname{Map}\left(A^{n}, A\right)$ defines an operad called the endomorphism operad of $A$. The group $\Sigma_{n}$ acts by permuting the factors in $A^{n}$.

Definition 2.12. An operad $\mathcal{P}$ is said to be an $E_{\infty}$-operad if for each $n$ the space $\mathcal{P}(n)$ is contractible and the action of $\Sigma_{n}$ on $\mathcal{P}(n)$ is free. 
An $E_{\infty}$-operad models multiplications which are associative and commutative up to all higher homotopies. The following example plays a central role in our work:

Definition 2.13. A universe is a countably infinite dimensional complex Hermitian vector space. Given a universe $\mathbb{H}$, the complex linear isometries operad $\mathscr{L}^{\mathbb{H}}$ over $\mathbb{H}$ has $\mathscr{L}^{\mathbb{H}}(n)$ the space of linear isometries $\mathbb{H}^{n} \rightarrow \mathbb{H}$. The group $\Sigma_{n}$ acts on $\mathscr{L}^{\mathbb{H}}(n)$ by $(\phi \sigma)(\mathbf{v})=\phi(\sigma \mathbf{v})$ where $\phi \in \mathscr{L}^{\mathbb{H}}(n)$ and $\mathbf{v}=\left(v_{1}, \ldots, v_{n}\right) \in \mathbb{H}^{n}$.

The action of $\Sigma_{n}$ on $\mathscr{L}^{\mathbb{H}}(n)$ is clearly free and the spaces $\mathscr{L}^{\mathbb{H}}(n)$ are contractible [34, Lemma 1.3] so $\mathscr{L}^{\mathbb{H}}$ is an $E_{\infty}$-operad.

2.2.1. Algebras over operads. An algebra $A$ over an operad $\mathcal{P}$, also called a $\mathcal{P}$ algebra, is a based topological space $A$ together with a sequence of equivariant maps $q: \mathcal{P}(n) \times A^{n} \rightarrow A$ which we represent by $\left(\theta, a_{1}, \ldots, a_{n}\right) \mapsto \theta \cdot\left(a_{1}, \ldots, a_{n}\right)$, such that $\mathbf{1}(a)=a$ and the following diagram commutes:

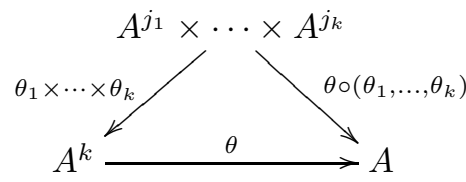

A morphism of $\mathcal{P}$-algebras is a map $f: A \rightarrow B$ such that $f\left(\theta \cdot\left(a_{1}, \ldots, a_{k}\right)\right)=$ $\theta \cdot\left(f\left(a_{1}\right), \ldots, f\left(a_{k}\right)\right)$.

2.2.2. Modules over algebras over operads. Let $\mathcal{P}$ be an operad and let $A$ be a $\mathcal{P}$ algebra. A left module over $A$ [21, section 1.6] is a topological space $M$ together with maps $q_{L}: \mathcal{P}(k+1) \times A^{k} \times M \rightarrow M$ (with $k \geq 0$ ), equivariant with respect to the action of $\Sigma_{k} \subset \Sigma_{k+1}$ (the subgroup of permutations preserving $k+1$ ), such that $q_{L}(\mathbf{1}, m)=m$ and the following diagram is commutative:

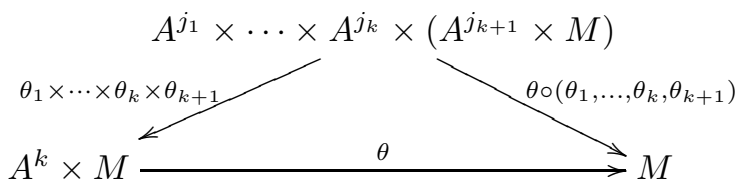

The above definition corresponds to the notion of a left module, but, using equivariance, a module $M$ over a $\mathcal{P}$-algebra $A$ can be equivalently defined 3 , Definition 1.1] as a family of maps $\mathcal{P}(n) \times A^{k-1} \times M \times A^{n-k} \rightarrow M$ satisfying suitable unit and associativity relations and inducing a total action

$$
q_{T}: \mathcal{P}(n) \times_{\Sigma_{n}}\left(\coprod_{k=1}^{n} A^{k-1} \times M \times A^{n-k}\right) \rightarrow M .
$$

In particular, for $k=1$ we have a map $\mathcal{P}(n) \times M \times A^{n-1} \rightarrow M$ giving $M$ the structure of a right module over the $\mathcal{P}$-algebra $A$.

Example 2.15. Let $\mathcal{P}$ be an operad. A morphism of $\mathcal{P}$-algebras $f: A \rightarrow B$ gives $B$ the structure of an $A$-module by defining

$$
q_{L}\left(\theta ; a_{1}, \ldots, a_{k}, b_{k+1}\right)=q\left(\theta ; f\left(a_{1}\right), \ldots, f\left(a_{k}\right), b_{k+1}\right) .
$$




\section{MOdUli SPACES OF HOLOMORPHIC BUNDLES}

In this section we give the moduli space of holomorphic bundles the structure of an algebra over the linear isometries operad (Definition 2.13) using the machinery of [5, 34].

Definition 3.1. Let $\mathscr{I}$ denote the category of linear isometries whose objects are the finite or countably infinite dimensional complex Hermitian vector spaces topologized as the limits of their finite dimensional subspaces and whose morphisms $\mathscr{I}(\mathbb{V}, \mathbb{W})$ are the linear isometries $\alpha: \mathbb{V} \rightarrow \mathbb{W}$. For each $r \geq 0$ let $\mathscr{I}_{r}$ be the full subcategory whose objects are the $r$-dimensional complex Hermitian vector spaces and let $\mathscr{I}_{*}$ be the union of the categories $\mathscr{I}_{r}$.

Recall that $L_{\infty} \subset \mathbb{P}^{2}$ denotes the rational curve at infinity and we write $\tilde{\mathbb{P}}_{I}^{2}$ for the blowup of $\mathbb{P}^{2}$ along a finite set $I \subset \mathbb{P}^{2} \backslash L_{\infty}$. We work with the following construction of the moduli space:

Definition 3.2. Let $\mathbb{V} \in$ ob $\mathscr{I}_{r}$ and let $E \rightarrow \tilde{\mathbb{P}}_{I}^{2}$ be a rank $r$ smooth complex vector bundle with first Chern class $c_{1}(E)=0$. A holomorphic structure on $E$ is a semi-connection $\bar{\partial}_{E}: \Omega^{0}(E) \rightarrow \Omega^{0,1}(E)$ satisfying the integrability condition $\bar{\partial}_{E}^{2}=0$. Let $\mathcal{C}(I, E, \mathbb{V})$ be the space of pairs $\left(\bar{\partial}_{E}, \phi\right)$ where $\bar{\partial}_{E}$ is a holomorphic structure on $E$ and $\phi:\left.E\right|_{L_{\infty}} \rightarrow \mathbb{V} \times L_{\infty}$ is a holomorphic trivialization. Let $\operatorname{Aut}(E)$ denote the group of smooth bundle automorphisms of $E$. Then we let $\mathfrak{M}(I, E, \mathbb{V})=\mathcal{C}(I, E, \mathbb{V}) / \operatorname{Aut}(E)$.

In [30. Theorem 1.1 and Lemma 2.6], it was shown that the group $\operatorname{Aut}(E)$ acts freely on $\mathcal{C}(I, E, \mathbb{V})$ and the quotient has the structure of a finite dimensional Hausdorff complex analytic space.

Proposition 3.3. Let $E_{1}, E_{2} \rightarrow \tilde{\mathbb{P}}_{I}^{2}$ be two isomorphic smooth complex vector bundles. Then there is a canonical isomorphism $\mathfrak{M}\left(I, E_{1}, \mathbb{V}\right) \cong \mathfrak{M}\left(I, E_{2}, \mathbb{V}\right)$.

Proof. Given an isomorphism $\psi: E_{1} \rightarrow E_{2}$ define a map $\psi_{*}: \mathcal{C}\left(I, E_{1}, \mathbb{V}\right) \rightarrow$ $\mathcal{C}\left(I, E_{2}, \mathbb{V}\right)$ by $\psi_{*}(\bar{\partial}, \phi)=\left(\psi \circ \bar{\partial} \circ \psi^{-1}, \phi \circ \psi^{-1}\right)$. This map descends to the quotient to give a homeomorphism $\mathfrak{M}\left(I, E_{1}, \mathbb{V}\right) \rightarrow \mathfrak{M}\left(I, E_{2}, \mathbb{V}\right)$ which is independent of the choice of isomorphism $\psi$.

Since the isomorphism class of $E$ is completely determined by $c_{2}(E)=k$ and $\operatorname{rk} E=\operatorname{dim} \mathbb{V}$, we will use the notation $\mathfrak{M}_{I, k}^{\mathbb{V}}=\mathfrak{M}(I, E, \mathbb{V})$.

Definition 3.4. Let $I \subset \mathbb{C}^{2}$ be a finite set.

(1) For each $\mathbb{V} \in$ ob $\mathscr{I}_{*}$ we represent the moduli space of holomorphic bundles on $\tilde{\mathbb{P}}_{I}^{2}$ with rank $r=\operatorname{dim} \mathbb{V}$ by

$$
\mathfrak{M}_{I}^{\mathbb{V}}=\coprod_{k=0}^{\infty} \mathfrak{M}_{I, k}^{\mathbb{V}},
$$

with base point the trivial bundle $\mathbb{V} \times \tilde{\mathbb{P}}_{I}^{2} \in \mathfrak{M}_{I, 0}^{\mathbb{V}}$.

(2) Let $\alpha \in \mathscr{I}_{r}\left(\mathbb{V}_{1}, \mathbb{V}_{2}\right)$. For each rank $r$ smooth bundle $E$ we define the map $\mathcal{C} \alpha: \mathcal{C}\left(I, E, \mathbb{V}_{1}\right) \rightarrow \mathcal{C}\left(I, E, \mathbb{V}_{2}\right)$ by sending $\left(\bar{\partial}_{E}, \phi\right)$ to $\left(\bar{\partial}_{E},(\alpha \times \mathrm{id}) \circ \phi\right)$. These maps descend to the quotient to give a map

$$
\mathfrak{M}_{I}^{\alpha}: \mathfrak{M}_{I}^{\mathbb{V}_{1}} \rightarrow \mathfrak{M}_{I}^{\mathbb{V}_{2}}
$$


(3) Given $\mathbb{V}_{1}, \mathbb{V}_{2} \in$ ob $\mathscr{I}_{*}$ and smooth bundles $E_{1}, E_{2}$ let $\omega: \mathcal{C}\left(I, E_{1}, \mathbb{V}_{1}\right) \times$ $\mathcal{C}\left(I, E_{2}, \mathbb{V}_{2}\right) \rightarrow \mathcal{C}\left(I, E_{1} \oplus E_{2}, \mathbb{V}_{1} \oplus \mathbb{V}_{2}\right)$ be the map defined by $\omega\left(\left(\bar{\partial}_{1}, \phi_{1}\right),\left(\bar{\partial}_{2}, \phi_{2}\right)\right)=$ $\left(\bar{\partial}_{1} \oplus \bar{\partial}_{2}, \phi_{1} \oplus \phi_{2}\right)$. These maps descends to the quotient to give a map

$$
\omega: \mathfrak{M}_{I}^{\mathbb{V}_{1}} \times \mathfrak{M}_{I}^{\mathbb{V}_{2}} \rightarrow \mathfrak{M}_{I}^{\mathbb{V}_{1} \oplus \mathbb{V}_{2}}
$$

(4) Given $J \subset I$ let $\pi_{J, I}: \tilde{\mathbb{P}}_{I}^{2} \rightarrow \tilde{\mathbb{P}}_{J}^{2}$ be the blowup of $\tilde{\mathbb{P}}_{J}^{2}$ along $I \backslash J$. Then, for each smooth bundle $E$, pullback of holomorphic bundles induces a map $\pi_{J, I}^{*}: \mathcal{C}(J, E, \mathbb{V}) \rightarrow \mathcal{C}\left(I, \pi_{J, I}^{*} E, \mathbb{V}\right)$. These maps descend to the quotient to give a map

$$
\pi_{J, I}^{*}: \mathfrak{M}_{J}^{\mathbb{V}} \rightarrow \mathfrak{M}_{I}^{\mathbb{V}} .
$$

Lemma 3.5. Let $\mathbb{V}, \mathbb{W} \in \mathrm{ob} \mathscr{I}_{*}$ and let $* \in \mathfrak{M}_{I}^{\mathbb{W}}$ be the basepoint. Then the map

$$
\omega \circ(\mathrm{id} \times *): \mathfrak{M}_{I}^{\mathbb{V}} \rightarrow \mathfrak{M}_{I}^{\mathbb{V} \oplus \mathbb{W}}
$$

is a closed embedding.

Proof. The map $\omega \circ(\mathrm{id} \times *)$ is given by Whitney sum with the trivial bundle $\mathbb{W} \times \tilde{\mathbb{P}}_{I}^{2}$. For each $k$, the moduli space $\mathfrak{M}_{I, k}^{\mathbb{V}}$ can be described [12, page 532] as the space of some matrices $a, a_{00}^{0}, a_{00}^{1}, c, d$ obeying the integrability condition in [12, equation (3.4)], modulo the free action of a certain group $G$ [12, equation (3.7)]. Here $c \in \operatorname{Hom}\left(\mathbb{C}^{k}, \mathbb{V}\right), d \in \operatorname{Hom}\left(\mathbb{V}, \mathbb{C}^{k}\right)$ and the group action on $c$ and $d$ is given by $c \mapsto c h_{00}$ and $d \mapsto g_{00} d$ with $h_{00}, g_{00} \in G L(k)$. The holomorphic bundle is given as the cohomology of a monad of the form (see [12, equation (3.1)]):

$$
0 \rightarrow \mathcal{E}_{0} \oplus \mathcal{E}_{1} \stackrel{A}{\rightarrow} W_{0} \oplus \mathbb{V} \stackrel{B}{\rightarrow} \mathcal{F}_{0} \oplus \mathcal{F}_{1} \rightarrow 0
$$

for some bundles $\mathcal{E}_{i}, \mathcal{F}_{i}$ over $\tilde{\mathbb{P}}_{I}^{2}$ and a vector space $W_{0}$. If $\left[z_{0}, z_{1}, z_{2}\right] \in \mathbb{P}^{2}$ are

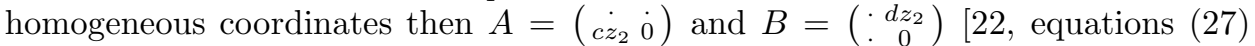
and (28)]. It follows that the map $\omega \circ$ (id $\times *$ ) is induced by the assignment $\left(a, a_{00}^{0}, a_{00}^{1}, c, d\right) \mapsto\left(a, a_{00}^{0}, a_{00}^{1}, \imath \circ c, d \circ p\right)$ where $\imath: \mathbb{V} \rightarrow \mathbb{V} \oplus \mathbb{W}$ is the inclusion and $p: \mathbb{V} \oplus \mathbb{W} \rightarrow \mathbb{V}$ is the projection. So $\omega \circ($ id $\times *)$ embeds $\mathfrak{M}_{I, k}^{\mathbb{V}}$ as a closed submanifold of $\mathfrak{M}_{I, k}^{\mathbb{V} \oplus \mathbb{W}}$.

Using the terminology of [34, the pair $\left(\mathfrak{M}_{I}, \omega\right)$ is an $\mathscr{I}_{*}$-functor. That is:

Proposition 3.6. Let $\oplus: \mathscr{I}_{*} \times \mathscr{I}_{*} \rightarrow \mathscr{I}_{*}$ be the direct sum functor. Then the assignments $\mathbb{V} \mapsto \mathfrak{M}_{I}^{\mathbb{V}}$ and $\alpha \mapsto \mathfrak{M}_{I}^{\alpha}$ define a continuous functor $\mathfrak{M}_{I}$ from $\mathscr{I}_{*}$ to Top and $\omega: \mathfrak{M}_{I} \times \mathfrak{M}_{I} \rightarrow \mathfrak{M}_{I} \circ \oplus$ is a commutative, associative and continuous natural transformation satisfying $\omega(x, *)=x$, (where $* \in \mathfrak{M}_{I}^{0}$ is the basepoint) and such that $\omega \circ(\mathrm{id} \times *)$ is a closed embedding.

Proof. The proof is straightforward.

Proposition 3.7. The functor $\mathfrak{M}_{I}$ extends to a functor $\mathfrak{M}_{I}: \mathscr{I} \rightarrow$ Top and $\omega$ extends to a natural transformation $\mathfrak{M}_{I} \times \mathfrak{M}_{I} \rightarrow \mathfrak{M}_{I} \circ \oplus$

Proof. We can extend $\mathfrak{M}_{I}$ to infinite dimensional vector spaces $\mathbb{H}$ by letting $\mathfrak{M}_{I}^{\mathbb{H}}=$ colim $\mathfrak{M}_{I}^{\mathbb{V}}$ where the colimit is taken over the finite dimensional subspaces $\mathbb{V} \subset \mathbb{H}$. See [34, Proposition 1.9] for details.

Proposition 3.8. Let $\mathscr{L}^{\mathbb{H}}$ be the complex linear isometries operad over a universe $\mathbb{H}$ (Definition [2.13). Then $\omega$ induces an $\mathscr{L}^{\mathbb{H}}$-algebra structure on $\mathfrak{M}_{I}^{\mathbb{H}}$. Furthermore, a linear isometric isomorphism $\alpha: \mathbb{H}_{1} \rightarrow \mathbb{H}_{2}$ induces a map of $\mathscr{L}^{\mathbb{H}_{1}}$-algebras $\mathfrak{M}_{I}^{\alpha}: \mathfrak{M}_{I}^{\mathbb{H}_{1}} \rightarrow \mathfrak{M}_{I}^{\mathbb{H}_{2}}$ which is a homeomorphism. 
Proof. See [34, Definition 1.6, Remark 1.7].

Given finite subsets $J \subset I \subset \mathbb{C}^{2}$, the map $\pi_{J, I}^{*}$ from Definition 3.4(4) passes to the colimit to give a map $\pi_{J, I}^{*}: \mathfrak{M}_{J}^{\mathbb{H}} \rightarrow \mathfrak{M}_{I}^{\mathbb{H}}$.

Definition 3.9. Let $\mathfrak{C}$ be the category of finite subsets of $\mathbb{C}^{2}$ with morphisms the inclusions.

Proposition 3.10. The assignments $I \mapsto \mathfrak{M}_{I}^{\mathbb{H}}$ and $(J \subset I) \mapsto \pi_{J, I}^{*}$ define a functor $\mathfrak{M}$ between $\mathfrak{C}$ and the category of $\mathscr{L}^{\mathbb{H}}$-algebras.

Proof. We can easily check that $\pi_{J, I}^{*}$ is a natural transformation between the functors $\mathfrak{M}_{I}$ and $\mathfrak{M}_{J}$ which commutes with $\omega$. The result follows.

Remark 3.11. For $J \subset I$, the space $\mathfrak{M}_{I}^{\mathbb{H}}$ has the structure of a module over the $\mathscr{L}^{\mathbb{H}_{-}}$ algebra $\mathfrak{M}_{J}^{\mathbb{H I}}$, induced by the map $\pi_{J, I}^{*}: \mathfrak{M}_{J}^{\mathbb{H}} \rightarrow \mathfrak{M}_{I}^{\mathbb{H}}$ and the $\mathscr{L}^{\mathbb{H}}$-algebra structure on $\mathfrak{M}_{I}^{\mathbb{H}}$ (see Example 2.15).

\section{The homotopy COHERENT BAR CONSTRUCtion}

We wish to generalize the bar construction of section 2.1.3 to the case where, instead of a monoid $G$ acting on spaces $X$ and $Y$, we have an algebra $A$ over an operad $\mathcal{P}$ and modules $M, N$ over $A$.

4.1. Modules. Let $\mathcal{P}$ be an operad and let $A$ be a $\mathcal{P}$-algebra. We need to generalize the notion of a (right) module $M$ over $A$ of section 2.2.2 to the case where the maps $M \times A^{n} \rightarrow M$ are parametrized, not by $\mathcal{P}(n+1)$, but by a certain sequence of spaces $\mathscr{M}(n)$. Recall the notation from Definition 2.9.

Definition 4.1. Let $\mathcal{P}$ be an operad with composition data $\gamma$ and unit $\mathbf{1} \in \mathcal{P}(1)$. A monoidal module $\mathscr{M}$ over $\mathcal{P}$ is a sequence of spaces $\mathscr{M}(n)$ (where $n \geq 0$ ) together with the following data: a right action of $\Sigma_{n}$ on $\mathscr{M}(n)$; a unit $\mathbf{1}_{\mathscr{M}} \in \mathscr{M}(0)$; and maps

$$
\Gamma_{R}: \mathscr{M}(k) \times \mathscr{M}\left(j_{0}\right) \times \mathcal{P}\left(j_{1}\right) \times \cdots \times \mathcal{P}\left(j_{k}\right) \rightarrow \mathscr{M}(j) \quad\left(j=j_{0}+\ldots+j_{k}\right),
$$

which we also represent by $\Gamma_{R}\left(\xi ; \xi^{\prime}, \theta_{1}, \ldots, \theta_{k}\right)=\xi \circ_{R}\left(\xi^{\prime}, \theta_{1}, \ldots, \theta_{k}\right)$, satisfying:

Equivariance: Let $\xi \in \mathscr{M}(k), \theta_{i} \in \mathcal{P}\left(j_{i}\right), \xi^{\prime} \in \mathscr{M}\left(j_{0}\right), \tau_{i} \in \Sigma_{j_{i}}$ and $\sigma \in \Sigma_{k+1}$ with $\sigma(0)=0$. Then

$$
\begin{aligned}
\xi \circ_{R}\left(\xi^{\prime} \tau_{0}, \theta_{1} \tau_{1}, \ldots, \theta_{k} \tau_{k}\right) & =\left(\xi \circ_{R}\left(\xi^{\prime}, \theta_{1}, \ldots, \theta_{k}\right)\right)\left(\tau_{0} \oplus \cdots \oplus \tau_{k}\right) \\
(\xi \sigma) \circ_{R}\left(\xi^{\prime}, \theta_{1}, \ldots, \theta_{k}\right) & =\left(\xi \circ_{R} \sigma\left(\xi^{\prime}, \theta_{1}, \ldots, \theta_{k}\right)\right) \sigma_{j_{0}, \ldots, j_{k}}
\end{aligned}
$$

\section{Associativity:}

$$
\begin{aligned}
\xi \circ_{R}\left(\xi^{\prime} \circ_{R}\left(\xi^{\prime \prime}, \theta_{01}, \ldots, \theta_{0 j_{0}}\right), \theta_{1} \circ\left(\theta_{11}, \ldots, \theta_{1 j_{1}}\right), \ldots, \theta_{k} \circ\left(\theta_{k 1}, \ldots, \theta_{k j_{k}}\right)\right) \\
=\left(\xi \circ_{R}\left(\xi^{\prime}, \theta_{1}, \ldots, \theta_{k}\right)\right) \circ_{R}\left(\xi^{\prime \prime}, \theta_{01}, \ldots, \theta_{0 j_{0}}, \ldots, \theta_{k 1}, \ldots, \theta_{k j_{k}}\right)
\end{aligned}
$$

Unit: $\mathbf{1}_{\mathscr{M}} \circ_{R} \xi=\xi \circ_{R}\left(\mathbf{1}_{\mathscr{M}}, \mathbf{1}, \ldots, \mathbf{1}\right)=\xi$.

We say $\mathscr{M}$ is an $E_{\infty}$-module if the $\Sigma_{k}$ actions are free and the spaces $\mathscr{M}(k)$ are contractible.

A morphism of monoidal modules $\mathscr{M}_{1} \rightarrow \mathscr{M}_{2}$ over an operad $\mathcal{P}$ is a sequence of equivariant maps $\mathscr{M}_{1}(n) \rightarrow \mathscr{M}_{2}(n)$ such that the induced map $\psi: \coprod \mathscr{M}_{1}(n) \rightarrow$ $\amalg \mathscr{M}_{2}(n)$ satisfies $\psi\left(\mathbf{1}_{\mathscr{M}}\right)=\mathbf{1}_{\mathscr{M}}$ and $\psi\left(\xi \circ_{R}\left(\xi^{\prime}, \theta_{1}, \ldots, \theta_{k}\right)\right)=(\psi \xi) \circ_{R}\left(\psi \xi^{\prime}, \theta_{1}, \ldots, \theta_{k}\right)$. 
Remark 4.2. The name monoidal module comes from $\mathscr{M}$ being a right module over $\mathcal{P}$ [18, section 5.1.1] and $\coprod_{n} \mathscr{M}(n)$ being a monoid.

Example 4.3. Given spaces $A$ and $M$ the sequence $\operatorname{End}_{A \mid M}(n)=\operatorname{Map}\left(M \times A^{n}, M\right)$ is a monoidal module over the endomorphisms operad End $A_{A}$ of Example 2.11.

We now generalize the definition of a module over a $\mathcal{P}$-algebra from section 2.2.2.

Definition 4.4. Let $\mathscr{M}$ be a monoidal module over an operad $\mathcal{P}$. A $\mathscr{M}$-module over a $\mathcal{P}$-algebra $A$ is a topological space $M$ together with $\Sigma_{k}$ equivariant maps $q_{R}: \mathscr{M}(k) \times M \times A^{k} \rightarrow M$, such that the following diagram is commutative:

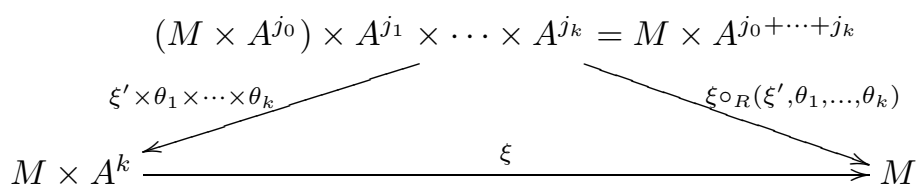

Example 4.5. Let $\mathcal{P}$ be an operad with composition data $\gamma$ and, for each $n \geq 0$, let $\mathcal{P}_{+}(n)=\mathcal{P}(n+1)$. Then $\mathcal{P}_{+}$is a monoidal module over $\mathcal{P}$ with data $\Gamma_{R}=\gamma$ and the $\mathcal{P}_{+}$-modules over a $\mathcal{P}$-algebra $A$ are the right modules over $A$ in the sense of section 2.2 .2

The main example of a monoidal module in our work is the following:

Example 4.6. Let $\mathscr{I}$ denote the category of linear isometries (Definition 3.1). Given universes $\mathbb{H}$ and $\mathbb{U}$ (Definition 2.13), let $\mathscr{M}(n)=\mathscr{I}\left(\mathbb{H} \oplus \mathbb{U}^{n}, \mathbb{H}\right)$. Then $\mathscr{M}(n)$ is a monoidal module over the linear isometries operad $\mathscr{L}^{\mathbb{U}}$. We are interested in the case where $\mathbb{U}=\mathbb{H}^{r}$ for some $r>0$. Let $\mathcal{P}^{r}(n)=\prod_{i=1}^{r} \mathscr{L}^{\mathbb{H}}(n)$. Then $\mathcal{P}^{r}(n)$ is naturally a sub-operad of $\mathscr{L}^{\mathbb{H}^{r}}(n)$ so, in this case, $\mathscr{M}$ is a monoidal module over $\mathcal{P}^{r}$. Furthermore, if $A$ in a $\mathscr{L}^{\mathbb{H}}$-algebra then $A^{r}$ is a $\mathcal{P}^{r}$-algebra and $A$ is a $\mathscr{M}$-module over $A^{r}$.

Remark 4.7. Definitions 4.1 and 4.4 correspond to the notion of a right module. In a completely analogous way we can also define left monoidal modules $\mathscr{N}$ over an operad $\mathcal{P}$ and left $\mathscr{N}$-modules over a $\mathcal{P}$-algebra $A$.

4.2. The bar construction. To define the homotopy coherent bar construction we replace the simplicial category $\Delta$ with a new category, obtained by expanding the spaces of morphisms to include the operad data. Given an operad $\mathcal{P}$, write $\mathcal{P}_{+}(n)=\mathcal{P}(n+1)($ see Example 4.5)

Definition 4.8. Let $\mathcal{P}$ be an operad with data $\gamma$ and let $\mathscr{M}$ be a monoidal module over $\mathcal{P}$ with composition data $\Gamma_{R}$. We represent by $\Delta(\mathscr{M}, \mathcal{P})$ the category with the same objects as the simplicial category $\Delta$ and whose morphisms are defined as follows: For each morphism $\mu \in \Delta(m, n)$ let

$$
\Delta(\mathscr{M}, \mathcal{P})(\mu)=\mathscr{M}\left(\mu_{0}\right) \times\left(\prod_{\alpha=1}^{m} \mathcal{P}\left(\mu_{\alpha}-\mu_{\alpha-1}\right)\right) \times \mathcal{P}_{+}\left(n-\mu_{m}\right) ;
$$

Then, the space of morphisms is defined to be:

$$
\Delta(\mathscr{M}, \mathcal{P})(m, n)=\coprod_{\mu \in \Delta(m, n)} \Delta(\mathscr{M}, \mathcal{P})(\mu) .
$$


Let $\mu \in \Delta(m, n)$ and $\nu \in \Delta(n, p)$. Composition of morphisms: $\Delta(\mathscr{M}, \mathcal{P})(\mu) \times$ $\Delta(\mathscr{M}, \mathcal{P})(\nu) \rightarrow \Delta(\mathscr{M}, \mathcal{P})(\nu \circ \mu)$ is defined using the operad data:

$$
\begin{aligned}
\Gamma_{R}: \mathscr{M}\left(\mu_{0}\right) \times \mathscr{M}\left(\nu_{0}\right) \times \prod_{\beta=1}^{\mu_{0}} \mathcal{P}\left(\nu_{\beta}-\nu_{\beta-1}\right) \rightarrow \mathscr{M}\left(\nu_{\mu_{0}}\right) \\
\gamma: \mathcal{P}\left(\mu_{\alpha}-\mu_{\alpha-1}\right) \times \prod_{\beta=\mu_{\alpha-1}+1}^{\mu_{\alpha}} \mathcal{P}\left(\nu_{\beta}-\nu_{\beta-1}\right) \rightarrow \mathcal{P}\left(\nu_{\mu_{\alpha}}-\nu_{\mu_{\alpha-1}}\right) \\
\gamma: \mathcal{P}_{+}\left(n-\mu_{m}\right) \times\left(\prod_{\beta=\mu_{m}+1}^{n} \mathcal{P}\left(\nu_{\beta}-\nu_{\beta-1}\right)\right) \times \mathcal{P}_{+}\left(p-\nu_{n}\right) \rightarrow \mathcal{P}_{+}\left(p-\nu_{\mu_{m}}\right) .
\end{aligned}
$$

From the associativity of the operad data it is straightforward to prove that this composition law is associative.

Remark 4.9. The above construction could also be done replacing $\mathcal{P}_{+}$by an arbitrary (left) monoidal module but we won't need that generality.

We now define the bar construction. Comparing with the bar construction for monoids in section 2.1 .3 , the category $\Delta(\mathscr{M}, \mathcal{P})$ replaces the simplicial category $\Delta$ and geometric realization is replaced by the homotopy colimit (section 2.1.5). By analogy with the notation for geometric realization, we often represent the homotopy colimit of a $\Delta(\mathscr{M}, \mathcal{P})^{\text {op }}$-diagram $X$ by

$$
\underset{\Delta(\mathscr{M}, \mathcal{P})^{\text {op }}}{\text { hcolim }} X=\|X\| \text {. }
$$

Definition 4.10. Let $\mathcal{P}, \mathscr{M}$ be as in Definition 4.8. Let $A$ be a $\mathcal{P}$-algebra, let $M$ be a right $\mathscr{M}$-module over $A$ and let $N$ be a left module over $A$. We represent by $\mathfrak{B}_{\bullet}(M, A, N): \Delta(\mathscr{M}, \mathcal{P})^{\text {op }} \rightarrow$ Top the functor defined on objects by

$$
\mathfrak{B}_{n}(M, A, N)=M \times A^{n} \times N
$$

and defined on morphisms as follows: Given $\mu \in \Delta(m, n)$ we can write (compare with equation (2.3)):

$$
M \times A^{n} \times N=\left(M \times A^{\mu_{0}}\right) \times\left(\prod_{\alpha=1}^{n} A^{\mu_{\alpha}-\mu_{\alpha-1}}\right) \times\left(A^{n-\mu_{m}} \times N\right) .
$$

Then the map $\Delta(\mathscr{M}, \mathcal{P})(\mu) \times M \times A^{n} \times N \rightarrow M \times A^{m} \times N$ is induced by the maps

$$
\begin{aligned}
q_{R} & : \mathscr{M}\left(\mu_{0}\right) \times M \times A^{\mu_{0}} \longrightarrow M ; \\
q & : \mathcal{P}\left(\mu_{\alpha}-\mu_{\alpha-1}\right) \times A^{\mu_{\alpha}-\mu_{\alpha-1}} \longrightarrow A ; \\
q & : \mathcal{P}_{+}\left(n-\mu_{m}\right) \times A^{n-\mu_{m}} \times N \longrightarrow N .
\end{aligned}
$$

We define the bar construction by taking the homotopy colimit:

$$
\mathfrak{B}(M, A, N)=\underset{\Delta(\mathscr{M}, \mathcal{P})^{\text {op }}}{\operatorname{hoc}} \mathfrak{\bullet}(M, A, N) .
$$

4.3. Maps from the bar construction. If $G$ is a monoid acting on spaces $X$ and $Z$, we can build a map from $\mathrm{B}(X, G, Z)$ to a space $Y$ from a sequence of maps $X \times G^{n} \times Z \rightarrow Y$ giving a map to the constant diagram $Y_{\bullet}$ (see Example2.2). When $X=G$ and $Y=Z$ this map is a homotopy equivalence [23, Proposition 3.1(5)]. In this section we generalize these results to the bar construction of section 4.2 . 
Let $\mathcal{P}, \mathscr{M}$, and $A, M, N$ be as in Definitions 4.8 and 4.10, We wish to define maps $\mathfrak{B}(M, A, N) \rightarrow Y$ for some space $Y$. The idea is to replace $Y$ with a homotopically equivalent space. We will need, for each $n \geq 0$, spaces $\mathcal{Y}(n)$ parametrizing maps $M \times A^{n} \times N \rightarrow Y$. Although Definition 4.13 below could be made for any spaces $\mathcal{Y}(n)$ satisfying suitable associativity relations, in this paper we will confine ourselves to the case where $\mathcal{Y}(n)=\mathscr{M}(n+1)$.

Definition 4.12. Let $\widetilde{\Delta}$ denote the category whose objects are the ordered sets $[n]=\{0,1, \ldots, n\} \subset \mathbb{Z}$ plus the empty set, which we represent by $[-1] \in \widetilde{\Delta}$, and whose morphisms are the order preserving maps.

The simplicial category $\Delta$ is a full subcategory of $\widetilde{\Delta}$. We will define a category $\widetilde{\Delta}(\mathscr{M}, \mathcal{P})$ equivalent to $\widetilde{\Delta}$ which contains $\Delta(\mathscr{M}, \mathcal{P})$ as a full subcategory.

\section{Definition 4.13.}

(1) Given a monoidal module $\mathscr{M}$ over an operad $\mathcal{P}$, let $\mathcal{P}_{+}(n)=\mathcal{P}(n+1)$ and let $\mathscr{M}_{+}(n)=\mathscr{M}(n+1)$. We define a category $\widetilde{\Delta}(\mathscr{M}, \mathcal{P})$ with the same objects as $\widetilde{\Delta}$ and morphisms defined as follows: For $m, n \neq[-1]$, the spaces of morphisms coincide with those of $\Delta(\mathscr{M}, \mathcal{P})$ and we let $\widetilde{\Delta}(\mathscr{M}, \mathcal{P})(-1, n)=$ $\mathscr{M}_{+}(n)$. Given $m, n \geq 0$ and $\mu \in \Delta(m, n)$, composition of morphisms

$$
\widetilde{\Delta}(\mathscr{M}, \mathcal{P})(-1, m) \times \widetilde{\Delta}(\mathscr{M}, \mathcal{P})(\mu) \rightarrow \widetilde{\Delta}(\mathscr{M}, \mathcal{P})(-1, n)
$$

is defined using the operad data:

$$
\Gamma_{R}: \mathscr{M}_{+}(m) \times \mathscr{M}\left(\mu_{0}\right) \times\left[\prod_{\alpha=1}^{m} \mathcal{P}\left(\mu_{\alpha}-\mu_{\alpha-1}\right)\right] \times \mathcal{P}_{+}\left(n-\mu_{m}\right) \rightarrow \mathscr{M}_{+}(n) .
$$

(2) Let $A$ be a $\mathcal{P}$-algebra, let $M$ be a right $\mathscr{M}$-module over $A$ and let $N$ be a left module over $A$. Let $Y$ be a topological space and suppose we have, for each $n \geq 0$, maps

$$
Q: \mathscr{M}_{+}(n) \times M \times A^{n} \times N \rightarrow Y
$$

equivariant with respect to the $\Sigma_{n}$-action on $\mathscr{M}_{+}(n)$ and $A^{n}$, and such that the following diagram commutes (where $j=j_{0}+\cdots+j_{k+1}$ ):

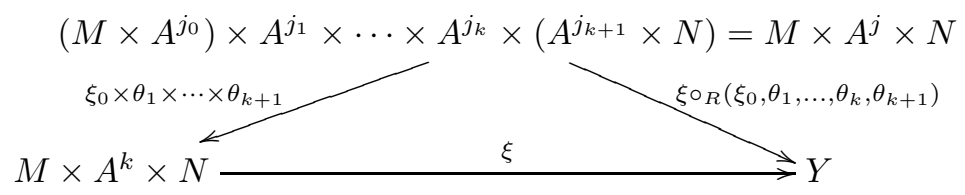

Let $\widetilde{\mathfrak{B}} \bullet(M, A, N ; Y): \widetilde{\Delta}(\mathscr{M}, \mathcal{P})^{\text {op }} \rightarrow$ Top be the functor extending $\mathfrak{B} \bullet(M, A, N)$, sending the object $[-1]$ to $Y$ and defined on the remaining morphisms by the map in equation (4.14). We let

$$
\widetilde{\mathfrak{B}}(M, A, N ; Y)=\underset{\Delta(\mathscr{M}, \mathcal{P})^{\text {op }}}{\operatorname{hcolim}} \widetilde{\mathfrak{B}}(M, A, N ; Y) .
$$

Proposition 4.16. The inclusion $[-1] \rightarrow \widetilde{\Delta}$ induces a map $Y \rightarrow \widetilde{\mathfrak{B}}(M, A, N ; Y)$ which is a homotopy equivalence.

Proof. This follows from the cofinality theorem [23, Proposition 4.4] since [-1] is a homotopy initial object. 
Definition 4.17. We represent by $h_{M, A, N ; Y}: \mathfrak{B}(M, A, N) \rightarrow Y$ the map in the homotopy category hTop determined by the diagram:

$$
\mathfrak{B}(M, A, N) \longrightarrow \widetilde{\mathfrak{B}}(M, A, N ; Y) \stackrel{\simeq}{\longleftarrow} Y .
$$

where the first map is determined by the functoriality of the homotopy colimit.

If $G$ is a monoid acting on the right on a space $Y$ and on the left on itself, then $\mathrm{B}(Y, G, G) \simeq Y$ [23, Proposition 3.1(5)]. The analogous result for $\mathfrak{B}$ is the following:

Proposition 4.18. Let $\mathscr{M}$ be a monoidal module over an operad $\mathcal{P}$ and let $M$ be a $\mathscr{M}$-module over a $\mathcal{P}$-algebra $A$. Then the map $h_{M, A, A ; M}: \mathfrak{B}(M, A, A) \rightarrow M$ is a homotopy equivalence. Furthermore, if $\mathscr{M}=\mathcal{P}_{+}$and $N$ is a left module over $A$ then the map $h_{A, A, N ; N}: \mathfrak{B}(A, A, N) \rightarrow N$ is a homotopy equivalence.

Proof. The statement for $h_{M, A, A ; M}$ follows immediately from Lemma A.6 in appendix $\mathrm{A}$ and the proof for $h_{A, A, N ; N}$ is completely analogous.

4.4. Morphisms. If, for each $i=1,2$, we have a monoid $G_{i}$ acting on spaces $X_{i}$ and $Z_{i}$, then a homomorphism $f: G_{1} \rightarrow G_{2}$ and equivariant maps $f_{X}: X_{1} \rightarrow X_{2}$ and $f_{Z}: Z_{1} \rightarrow Z_{2}$ induce a natural transformation B. $\left(X_{1}, G_{1}, Z_{1}\right) \rightarrow \mathrm{B} \bullet\left(X_{2}, G_{2}, Z_{2}\right)$ and hence a map between the bar constructions which is a homotopy equivalence if $f, f_{X}$ and $f_{Z}$ are homotopy equivalences [23, Proposition 3.1(6)]. In this section we prove analogous results for the homotopy coherent bar construction.

Suppose $\psi: \mathcal{P}_{1} \rightarrow \mathcal{P}_{2}$ is a morphism of operads, and $\mathscr{M}$ is a monoidal module over $\mathcal{P}_{2}$ with composition data $\Gamma_{R}$. We represent by $\psi^{*} \mathscr{M}$ the monoidal module over $\mathcal{P}_{1}$ with $\psi^{*} \mathscr{M}(n)=\mathscr{M}(n)$ and composition data:

$$
\psi^{*} \Gamma_{R}\left(\xi ; \xi^{\prime}, \theta_{1}, \ldots, \theta_{n}\right)=\Gamma_{R}\left(\xi ; \xi^{\prime}, \psi \theta_{1}, \ldots, \psi \theta_{n}\right) .
$$

For each $i=1,2$ let $\mathscr{M}_{i}$ be a monoidal module over $\mathcal{P}_{i}$ and let $\psi_{\mathscr{M}}: \mathscr{M}_{1} \rightarrow \psi^{*} \mathscr{M}_{2}$ be a morphism of monoidal modules over $\mathcal{P}_{1}$. Then $\psi, \psi_{\mathscr{M}}$ induce a functor $F: \Delta\left(\mathscr{M}_{1}, \mathcal{P}_{1}\right) \rightarrow \Delta\left(\mathscr{M}_{2}, \mathcal{P}_{2}\right)$ and given any $\Delta\left(\mathscr{M}_{2}, \mathcal{P}_{2}\right)$-diagram $\mathfrak{B}$ we have a pullback $\Delta\left(\mathscr{M}_{1}, \mathcal{P}_{1}\right)$-diagram $F^{*} \mathfrak{B}=F \circ \mathfrak{B}$. Now suppose $A$ is a $\mathcal{P}_{2}$-algebra and $M$ and $N$ are, respectively, a right $\mathscr{M}_{2}$-module over $A$ and a left module over $A$. Then we have a $\mathcal{P}_{1}$-algebra $\psi^{*} A$, a right $\mathscr{M}_{1}$-module $\psi^{*} M$ and a left module $\psi^{*} N$ over $\psi^{*} A$ defined in the obvious way.

Proposition 4.19. We have an isomorphism of $\Delta\left(\mathscr{M}_{1}, \mathcal{P}_{1}\right)^{\mathrm{op}}$-diagrams

$$
\mathfrak{B}_{\bullet}\left(\psi^{*} M, \psi^{*} A, \psi^{*} N\right) \cong F^{*} \mathfrak{B} \bullet(M, A, N)
$$

and if $\mathcal{P}_{i}, \mathscr{M}_{i}$ are $E_{\infty}$ then the functor $F$ induces a homotopy equivalence

$$
\operatorname{hcolim}_{\Delta\left(\mathscr{M}_{1}, \mathcal{P}_{1}\right)^{\text {op }}} F^{*} \mathfrak{B} \bullet(M, A, N) \stackrel{\simeq}{\longrightarrow} \operatorname{hcolim}_{\Delta\left(\mathscr{M}_{2}, \mathcal{P}_{2}\right)^{\text {op }}} \mathfrak{B} \bullet(M, A, N) .
$$

Proof. The isomorphism of $\Delta\left(\mathscr{M}_{1}, \mathcal{P}_{1}\right)^{\text {op }}$-diagrams can easily be checked by direct inspection. The homotopy equivalence follows from [23, Proposition 3.1(6)], since $F$ is an equivalence of categories.

Let $\mathcal{P}$ be an operad and let $\mathscr{M}$ be a monoidal module over $\mathcal{P}$. For each $i=1,2$ let $A_{i}$ be a $\mathcal{P}$-algebra, let $M_{i}$ be a $\mathscr{M}$-module over $A_{i}$ and let $N_{i}$ be a module over $A_{i}$. Let

$$
f: A_{1} \rightarrow A_{2}, \quad f_{M}: M_{1} \rightarrow f^{*} M_{2}, \quad f_{N}: N_{1} \rightarrow f^{*} N_{2}
$$


be, respectively, a morphism of $\mathcal{P}$ algebras, a morphism of $\mathscr{M}$-modules over $A_{1}$ and a morphism of modules over $A_{1}$. Also suppose we have, for each $i=1,2$, maps $Q_{i}: \mathscr{M}_{+}(k) \times M_{i} \times A_{i}^{k} \times N_{i} \rightarrow Y_{i}$ satisfying the associativity conditions in Definition 4.13 and let $f_{Y}: Y_{1} \rightarrow Y_{2}$ be a map such that

$$
f_{Y}\left(Q_{1}\left(\xi ; m, a_{1}, \ldots, a_{k}, n\right)\right)=Q_{2}\left(\xi ; f_{M}(m), f\left(a_{1}\right), \ldots, f\left(a_{k}\right), f_{N}(n)\right) .
$$

Then $f, f_{M}, f_{N}$ and $f_{Y}$ induce natural transformations, which we represent by

$$
\begin{aligned}
& \mathfrak{B}_{\bullet}\left(f_{M}, f, f_{N}\right): \mathfrak{B}_{\bullet}\left(M_{1}, A_{1}, N_{1}\right) \rightarrow \mathfrak{B}_{\bullet}\left(M_{2}, A_{2}, N_{2}\right) \text {; } \\
& \widetilde{\mathfrak{B}} \bullet\left(f_{M}, f, f_{N} ; f_{Y}\right): \widetilde{\mathfrak{B}} \bullet\left(M_{1}, A_{1}, N_{1} ; Y_{1}\right) \rightarrow \widetilde{\mathfrak{B}} \bullet\left(M_{2}, A_{2}, N_{2} ; Y_{2}\right) \text {. }
\end{aligned}
$$

Proposition 4.21. If $\mathcal{P}$ and $\mathscr{M}$ are $E_{\infty}$ and $f, f_{M}$ and $f_{N}$ are homotopy equivalences, then $\mathfrak{B}\left(f_{M}, f, f_{N}\right)$ is a homotopy equivalence.

Proof. This follows immediately from the homotopy invariance of the homotopy colimit [23, Proposition 4.4]

\section{The SPACES $\left\|\mathfrak{B}_{I}\right\|$}

Let $\mathscr{L}^{\mathbb{H}}$ be the linear isometries operad (Definition 2.13) and let $\mathscr{I}$ be the category of linear isometries (Definition 3.1). By Proposition 3.8, the spaces $\mathfrak{M}_{I}^{\mathbb{H}}$ are $\mathscr{L}^{\mathbb{H}}$-algebras. Definition 5.1. Given a finite set $I \subset \mathbb{C}^{2}$ let $\mathbb{H}^{I}=\bigoplus_{x \in I} \mathbb{H}$ and, for each non-negative
integer $n$, let

$$
\mathscr{L}_{I}^{\mathbb{H}}(n)=\mathscr{I}\left(\mathbb{H} \oplus\left(\mathbb{H}^{I}\right)^{n}, \mathbb{H}\right), \quad \quad \mathscr{L}^{\mathbb{H}, I}(n)=\prod_{x \in I} \mathscr{L}^{\mathbb{H}}(n) .
$$

Also, let $\mathscr{L}_{I+}^{\mathbb{H}}(n)=\mathscr{L}_{I}^{\mathbb{H}}(n+1)$ and $\mathscr{L}_{+}^{\mathbb{H}, I}(n)=\mathscr{L}^{\mathbb{H}, I}(n+1)$.

In Example 4.6 we observed that $\mathscr{L}_{I}^{\mathbb{H}}$ is a monoidal module over $\mathscr{L}^{\mathbb{H}, I}$ and $\mathfrak{M}_{\emptyset}^{\mathbb{H}}$ is a $\mathscr{L}_{I}^{\mathbb{H}}$-module over the $\mathscr{L}^{\mathbb{H}, I}$-algebra $\prod_{x \in I} \mathfrak{M}_{\emptyset}^{\mathbb{H}}$. The space $\prod_{x \in I} \mathfrak{M}_{x}^{\mathbb{H}}$ is also a $\mathscr{L}^{\mathbb{H}, I_{-}}$ algebra, and the pullback maps $\pi_{\emptyset, x}: \mathfrak{M}_{\emptyset}^{\mathbb{H}} \rightarrow \mathfrak{M}_{x}^{\mathbb{H}}$ make $\prod_{x \in I} \mathfrak{M}_{x}^{\mathbb{H}}$ into a module over $\prod_{x \in I} \mathfrak{M}_{\emptyset}^{\mathbb{H}}$ (Example 2.15). Next we apply Definition 4.13 (2) with $Y=\mathfrak{M}_{I}^{\mathbb{H}}$. We need a map

$$
Q: \mathscr{L}_{I+}^{\mathbb{H}}(n) \times \mathfrak{M}_{\emptyset}^{\mathbb{H}} \times\left(\prod_{x \in I} \mathfrak{M}_{\emptyset}^{\mathbb{H}}\right)^{n} \times \prod_{x \in I} \mathfrak{M}_{x}^{\mathbb{H}} \rightarrow \mathfrak{M}_{I}^{\mathbb{H}}
$$

satisfying the associativity conditions in Definition 4.13. We define this map, for each $f \in \mathscr{L}_{I+}^{\mathbb{H}}(n)$ by the composition:

$\mathfrak{M}_{\emptyset} \times\left(\prod \mathfrak{M}_{\emptyset}\right)^{n} \times\left(\prod \mathfrak{M}_{x}\right) \stackrel{\pi^{*}}{\rightarrow} \mathfrak{M}_{I} \times\left(\prod \mathfrak{M}_{I}\right)^{n} \times\left(\prod \mathfrak{M}_{I}\right)=\mathfrak{M}_{I} \times\left(\prod \mathfrak{M}_{I}\right)^{n+1} \stackrel{f}{\rightarrow} \mathfrak{M}_{I}$, where the map $\pi^{*}$ is induced by pullback.

Definition 5.2. We write:

$$
\begin{array}{ll}
\Delta_{I}^{\mathbb{H}}=\Delta\left(\mathscr{L}_{I}^{\mathbb{H}}, \mathscr{L}^{\mathbb{H}, I}\right) ; & \widetilde{\Delta}_{I}^{\mathbb{H}}=\widetilde{\Delta}\left(\mathscr{L}_{I}^{\mathbb{H}}, \mathscr{L}^{\mathbb{H}, I}\right) ; \\
\mathfrak{B}_{I}^{\mathbb{H}}=\mathfrak{B} \bullet\left(\mathfrak{M}_{\emptyset}^{\mathbb{H}}, \prod_{x \in I} \mathfrak{M}_{\emptyset}^{\mathbb{H}}, \prod_{x \in I} \mathfrak{M}_{x}^{\mathbb{H}}\right) ; & \widetilde{\mathfrak{B}}_{I}^{\mathbb{H}}=\widetilde{\mathfrak{B}}_{\bullet}\left(\mathfrak{M}_{\emptyset}^{\mathbb{H}}, \prod_{x \in I} \mathfrak{M}_{\emptyset}^{\mathbb{H}}, \prod_{x \in I} \mathfrak{M}_{x}^{\mathbb{H}} ; \mathfrak{M}_{I}^{\mathbb{H}}\right) .
\end{array}
$$

Also, we write $\left\|\mathfrak{B}_{I}^{\mathbb{H}}\right\|=$ hcolim $\mathfrak{B}_{I}^{\mathbb{H}}$ and we denote the map of Definition 4.17 by

$$
h_{I}:\left\|\mathfrak{B}_{I}^{\mathbb{H}}\right\| \rightarrow \mathfrak{M}_{I}^{\mathbb{H}} .
$$

Proposition 5.4. The maps $h_{\emptyset}, h_{x}$ are homotopy equivalences. 
Proof. This immediately follows from Proposition 4.18 ,

Let $\mathfrak{C}$ be as in Definition 3.9. Given a morphism $i: J \rightarrow I$ in $\mathfrak{C}$, the projection $\mathscr{L}^{\mathbb{H}, I}(n) \rightarrow \mathscr{L}^{\mathbb{H}, J}(n)$ and the map $\mathscr{L}_{I}^{\mathbb{H}}(n) \rightarrow \mathscr{L}_{J}^{\mathbb{H}}(n)$ induced by the inclusion $\mathbb{H} \oplus\left(\mathbb{H}^{J}\right)^{n} \rightarrow \mathbb{H} \oplus\left(\mathbb{H}^{I}\right)^{n}$ induce equivalences of categories, which we represent by

$$
\Delta_{i}: \Delta_{I}^{\mathbb{H}} \rightarrow \Delta_{J}^{\mathbb{H}} \quad \text { and } \quad \widetilde{\Delta}_{i}: \widetilde{\Delta}_{I}^{\mathbb{H}} \rightarrow \widetilde{\Delta}_{J}^{\mathbb{H}}
$$

and hence, by Proposition 4.19, homotopy equivalences

$$
\left\|\Delta_{i}^{*} \mathfrak{B}_{J}^{\mathbb{H}}\right\| \rightarrow\left\|\mathfrak{B}_{J}^{\mathbb{H}}\right\| \quad \text { and } \quad\left\|\widetilde{\Delta}_{i}^{*} \widetilde{\mathfrak{B}}_{J}^{\mathbb{H}}\right\| \rightarrow\left\|\widetilde{\mathfrak{B}}_{J}^{\mathbb{H}}\right\| .
$$

The inclusions of based spaces:

$$
i: \prod_{x \in J} \mathfrak{M}_{\emptyset}^{\mathbb{H}} \rightarrow \prod_{x \in I} \mathfrak{M}_{\emptyset}^{\mathbb{H}}, \quad \quad i_{N}: \prod_{x \in J} \mathfrak{M}_{x}^{\mathbb{H}} \rightarrow \prod_{x \in I} \mathfrak{M}_{x}^{\mathbb{H}},
$$

together with the pullback maps $\pi_{J, I}^{*}: \mathfrak{M}_{J}^{\mathbb{H}} \rightarrow \mathfrak{M}_{I}^{\mathbb{H}}$ induce maps (see equation 4.20)

$$
\begin{aligned}
\widetilde{\mathfrak{B}}\left(\mathrm{id}, i, i_{N} ; \pi_{J, I}^{*}\right):\left\|\widetilde{\Delta}_{i}^{*} \widetilde{\mathfrak{B}}_{J}^{\mathbb{H}}\right\| & \rightarrow\left\|\widetilde{\mathfrak{B}}_{I}^{\mathbb{H}}\right\| \\
\mathfrak{B}\left(\mathrm{id}, i, i_{N}\right):\left\|\Delta_{i}^{*} \mathfrak{B}_{J}^{\mathbb{H}}\right\| & \rightarrow\left\|\mathfrak{B}_{I}^{\mathbb{H}}\right\|
\end{aligned}
$$

Remark 5.7. Although we are not going to need these facts, we observe that the assignments $I \mapsto \Delta_{I}$ and $i \mapsto \Delta_{i}$ define a functor from $\mathfrak{C}^{\text {op }}$ to the category of topological categories equivalent to $\Delta$, and the assignments $I \mapsto\left\|\mathfrak{B}_{I}^{\mathbb{H}}\right\|$ and $(i: J \rightarrow$ $I) \mapsto\left\|\mathfrak{B}_{i}^{\mathbb{H}}\right\|$ define a functor $\left\|\mathfrak{B}^{\mathbb{H}}\right\|: \mathfrak{C} \rightarrow$ hTop. Furthermore the maps $h_{I}:\left\|\mathfrak{B}_{I}^{\mathbb{H}}\right\| \rightarrow$ $\mathfrak{M}_{I}^{\mathbb{H}}$ define a natural transformation between the functors $\left\|\mathfrak{B}^{\mathbb{H}}\right\|, \mathfrak{M}^{\mathbb{H}}: \mathfrak{C} \rightarrow \underline{\text { hTop }}$ (for the functor $\mathfrak{M}^{\mathbb{H}}$ see Proposition 3.10).

We now show that, for an appropriate choice of universes (Definition 2.13), the spaces $\left\|\widetilde{\mathfrak{B}}_{I}\right\|$ and $\left\|\mathfrak{B}_{I}\right\|$ are modules over the $\mathscr{L}$-algebra $\mathfrak{M}_{\emptyset}$ (see Proposition 5.8 below). Given universes $\mathbb{H}_{0}, \mathbb{H}_{1}$, we have canonical operad maps $i_{j}: \mathscr{L}^{\mathbb{H}_{j}} \rightarrow \mathscr{L}^{\mathbb{H}_{0} \otimes \mathbb{H}_{1}}$ (with $j=0,1$ ): the map $i_{0}$ maps $f \in \mathscr{L}^{\mathbb{H}_{0}}(n)$ to the isometry $f \otimes \mathbf{1}:\left(\mathbb{H}_{0}^{\oplus n}\right) \otimes \mathbb{H}_{1} \rightarrow$ $\mathbb{H}_{0} \otimes \mathbb{H}_{1}$ and similarly for $i_{1}$. Let $\mathbb{H}=\mathbb{H}_{0} \otimes \mathbb{H}_{1}$. The maps $i_{0}, i_{1}$ induce equivalences of categories $F_{i_{j}}: \Delta_{I}^{\mathbb{H}_{j}} \rightarrow \Delta_{I}^{\mathbb{H}}$ (with $j=0,1$ ), and by Proposition 4.19, homotopy equivalences

$$
\underset{\left(\Delta_{I}^{\mathbb{H}_{j}}\right)^{\mathrm{op}}}{\operatorname{col} \lim _{i_{j}}} F_{I}^{*} \mathfrak{B}_{\left(\Delta_{I}^{\mathbb{H}}\right)^{\mathrm{HP}}}^{\simeq} \underset{\operatorname{hcolim}}{\longrightarrow} \mathfrak{B}_{I}^{\mathbb{H}} .
$$

Also observe that each $i_{j}$ gives $\mathfrak{M}_{\emptyset}^{\mathbb{H}}$ the structure of a $\mathscr{L}^{\mathbb{H}_{j}}$-algebra.

Proposition 5.8. Let $\mathbb{H}_{\emptyset}, \mathbb{H}_{I}$ be universes, let $\mathbb{H}=\mathbb{H}_{\emptyset} \otimes \mathbb{H}_{I}$ and let $i_{I}: \mathscr{L}^{\mathbb{H}_{I}} \rightarrow \mathscr{L}^{\mathbb{H}}$ be the canonical map of operads. Then $\left\|F_{i_{I}}^{*} \widetilde{\mathfrak{B}}_{I}^{\mathbb{H}}\right\|$ and $\left\|F_{i_{I}}^{*} \mathfrak{B}_{I}^{\mathbb{H}}\right\|$ are left modules over the $\mathscr{L}^{\mathbb{H}_{\emptyset}}$-algebra $\mathfrak{M}_{\emptyset}^{\mathbb{H}}$.

Proof. Given $f \in \mathscr{L}_{+}^{\mathbb{H}} \mathbb{H}_{\emptyset}(n)$ the map $\left(\mathfrak{M}_{\emptyset}^{\mathbb{H}}\right)^{n} \times\left\|F_{i_{I}}^{*} \widetilde{\mathfrak{B}}_{I}^{\mathbb{H}}\right\| \rightarrow\left\|F_{i_{I}}^{*} \widetilde{\mathfrak{B}}_{I}^{\mathbb{H}}\right\|$ is defined by the natural transformation $\left(\mathfrak{M}_{\emptyset}^{\mathbb{H}}\right)^{n} \times F_{i_{I}}^{*} \widetilde{\mathfrak{B}}_{I}^{\mathbb{H}} \rightarrow F_{i_{I}}^{*} \widetilde{\mathfrak{B}}_{I}^{\mathbb{H}}$ given, for $m \neq-1$, by the maps:

$$
\begin{aligned}
\left(\mathfrak{M}_{\emptyset}^{\mathbb{H}}\right)^{n} \times \widetilde{\mathfrak{B}}_{I}^{\mathbb{H}}(m)=\left(\left(\mathfrak{M}_{\emptyset}^{\mathbb{H}}\right)^{n} \times \mathfrak{M}_{\emptyset}^{\mathbb{H}}\right) \times\left(\prod_{x \in I} \mathfrak{M}_{\emptyset}^{\mathbb{H}}\right)^{m} \times\left(\prod_{x \in I} \mathfrak{M}_{x}^{\mathbb{H}}\right) \\
\stackrel{\stackrel{i_{\emptyset}(f) \times \mathrm{id} \times \mathrm{id}}{\longrightarrow}}{\longrightarrow} \mathfrak{M}_{\emptyset}^{\mathbb{H}} \times\left(\prod_{x \in I} \mathfrak{M}_{\emptyset}^{\mathbb{H}}\right)^{m} \times\left(\prod_{x \in I} \mathfrak{M}_{x}^{\mathbb{H}}\right)=\widetilde{\mathfrak{B}}_{I}^{\mathbb{H}}(m)
\end{aligned}
$$


and for $m=-1$ by the maps

$$
\left(\mathfrak{M}_{\emptyset}^{\mathbb{H}}\right)^{n} \times \widetilde{\mathfrak{B}}_{I}^{\mathbb{H}}(-1)=\left(\mathfrak{M}_{\emptyset}^{\mathbb{H}}\right)^{n} \times \mathfrak{M}_{I}^{\mathbb{H}} \stackrel{\left(\pi_{\emptyset, I}^{*}\right)^{n} \times \mathrm{id}}{\longrightarrow}\left(\mathfrak{M}_{I}^{\mathbb{H}}\right)^{n+1} \stackrel{i_{\emptyset}(f)}{\longrightarrow} \mathfrak{M}_{I}^{\mathbb{H}}=\widetilde{\mathfrak{B}}_{I}^{\mathbb{H}}(-1) .
$$

The fact that this is a natural transformation follows from the commutativity of the following diagram, where $f \in \mathscr{L}^{\mathbb{H}_{\emptyset}}(n+1), g \in \mathscr{L}^{\mathbb{H}_{I}}(k+1)$ and $J=\emptyset$ or $J=I$ :

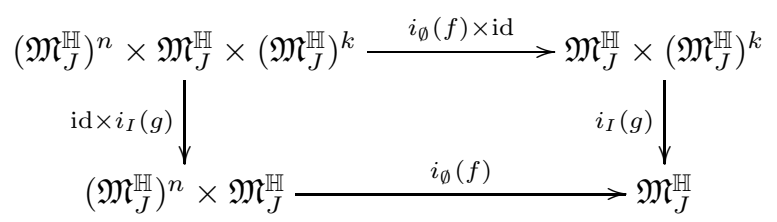

The map $\left(\mathfrak{M}_{\emptyset}^{\mathbb{H}}\right)^{n} \times\left\|F_{i_{I}}^{*} \mathfrak{B}_{I}^{\mathbb{H}}\right\| \rightarrow\left\|F_{i_{I}}^{*} \mathfrak{B}_{I}^{\mathbb{H}}\right\|$ is obtained by restricting the natural transformation defined above.

Remark 5.10. Using equivariance, we can also think of $\left\|F_{i_{I}}^{*} \widetilde{\mathfrak{B}}_{I}^{\mathbb{H}}\right\|$ and $\left\|F_{i_{I}}^{*} \mathfrak{B}_{I}^{\mathbb{H}}\right\|$ as right modules over $\mathfrak{M}_{\emptyset}^{\mathbb{H}}$ (see section 2.2.2). These structures are induced by the maps (compare with equation (5.9)):

$$
\begin{aligned}
\mathfrak{B}_{I}^{\mathbb{H}}(m) \times\left(\mathfrak{M}_{\emptyset}^{\mathbb{H}}\right)^{n} \stackrel{\text { shuffle }}{\longrightarrow} & \left(\mathfrak{M}_{\emptyset}^{\mathbb{H}} \times\left(\mathfrak{M}_{\emptyset}^{\mathbb{H}}\right)^{n}\right) \times\left(\prod_{x \in I} \mathfrak{M}_{\emptyset}^{\mathbb{H}}\right)^{m} \times\left(\prod_{x \in I} \mathfrak{M}_{x}^{\mathbb{H}}\right) \\
& \stackrel{i_{\emptyset}(f) \times \mathrm{id} \times \mathrm{id}}{\longrightarrow} \mathfrak{M}_{\emptyset}^{\mathbb{H}} \times\left(\prod_{x \in I} \mathfrak{M}_{\emptyset}^{\mathbb{H}}\right)^{m} \times\left(\prod_{x \in I} \mathfrak{M}_{x}^{\mathbb{H}}\right)=\mathfrak{B}_{I}^{\mathbb{H}}(m)
\end{aligned}
$$

and also, in the case of $\left\|F_{i_{I}}^{*} \widetilde{\mathfrak{B}}_{I}^{\mathbb{H}}\right\|$, by the maps

$$
\widetilde{\mathfrak{B}}_{I}^{\mathbb{H}}(-1) \times\left(\mathfrak{M}_{\emptyset}^{\mathbb{H}}\right)^{n}=\mathfrak{M}_{I}^{\mathbb{H}} \times\left(\mathfrak{M}_{\emptyset}^{\mathbb{H}}\right)^{n} \stackrel{\operatorname{id} \times\left(\pi_{\emptyset, I}^{*}\right)^{n}}{\longrightarrow}\left(\mathfrak{M}_{I}^{\mathbb{H}}\right)^{n+1} \stackrel{i_{\emptyset}(f)}{\longrightarrow} \mathfrak{M}_{I}^{\mathbb{H}}=\widetilde{\mathfrak{B}}_{I}^{\mathbb{H}}(-1) .
$$

Let $I, J \subset \mathbb{C}^{2}$ be finite disjoint sets. Fix universes $\mathbb{H}_{I J}, \mathbb{H}_{\emptyset}$ and let $\mathbb{H}=\mathbb{H}_{I J} \otimes \mathbb{H}_{\emptyset}$. Let $i: \mathscr{L}^{\mathbb{H}_{I J}} \rightarrow \mathscr{L}^{\mathbb{H}}$ be the canonical operad map. From Definition 4.10, with $\mathcal{P}=\mathscr{L}^{\mathbb{H}_{\emptyset}}$ and $\mathscr{M}=\mathcal{P}_{+}$(see Example 4.5), we get functors

$$
\begin{aligned}
& \mathfrak{B}_{\bullet}^{\mathbb{H}_{\emptyset}}\left(\left\|F_{i}^{*} \mathfrak{B}_{I}^{\mathbb{H}}\right\|, \mathfrak{M}_{\emptyset}^{\mathbb{H}},\left\|F_{i}^{*} \mathfrak{B}_{J}^{\mathbb{H}}\right\|\right): \Delta\left(\mathscr{L}_{+}^{\mathbb{H}_{\emptyset}}, \mathscr{L}^{\mathbb{H}_{\emptyset}}\right) \rightarrow \underline{\text { Top }}, \\
& \mathfrak{B}_{\bullet}^{\mathbb{H}_{\emptyset}}\left(\left\|F_{i}^{*} \widetilde{\mathfrak{B}}_{I}^{\mathbb{H}}\right\|, \mathfrak{M}_{\emptyset}^{\mathbb{H}},\left\|F_{i}^{*} \widetilde{\mathfrak{B}}_{J}^{\mathbb{H}}\right\|\right): \Delta\left(\mathscr{L}_{+}^{\mathbb{H}_{\emptyset}}, \mathscr{L}^{\mathbb{H}_{\emptyset}}\right) \rightarrow \underline{\text { Top }},
\end{aligned}
$$

where the superscript in $\mathfrak{B}_{\bullet}^{\mathbb{H} \emptyset}(\ldots)$ is added to stress that we are over the operad $\mathscr{L}^{\mathbb{H}_{\emptyset}}$. From Remark $3.11, \mathfrak{M}_{I}^{\mathbb{H}}$ and $\mathfrak{M}_{J}^{\mathbb{H}}$ are modules over the $\mathscr{L}^{\mathbb{H}}$-algebra $\mathfrak{M}_{\emptyset}^{\mathbb{H}}$. Taking $\mathcal{P}=\mathscr{L}^{\mathbb{H}}$ and $\mathscr{M}=\mathcal{P}_{+}$, we get the functor

$$
\mathfrak{B}_{\bullet}^{\mathbb{H}}\left(\mathfrak{M}_{I}^{\mathbb{H}}, \mathfrak{M}_{\emptyset}^{\mathbb{H}}, \mathfrak{M}_{J}^{\mathbb{H}}\right): \Delta\left(\mathscr{L}_{+}^{\mathbb{H}}, \mathscr{L}^{\mathbb{H}}\right) \rightarrow \underline{\text { Top }} .
$$

We will use the notation in equation (4.11) to represent the homotopy colimit of these functors.

Proposition 5.12. With notation as above, we have a commutative diagram in hTop:

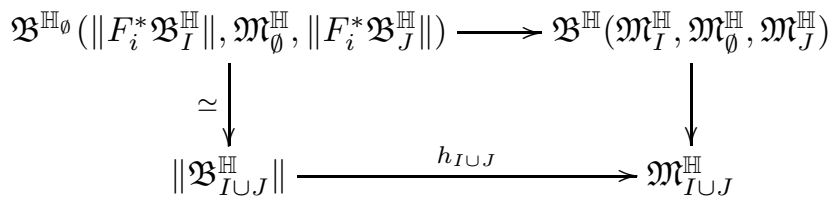


where the left vertical map is a homotopy equivalence and the top horizontal map is induced by $h_{I}$ and $h_{J}$.

The proof will be given on page 63 in appendix $\mathrm{A}$

\section{Proof of Theorem 1.3}

In this section we fix a universe $\mathbb{H}$, and write $\mathfrak{M}, \mathfrak{B}$ instead of $\mathfrak{M}^{\mathbb{H}}, \mathfrak{B}^{\mathbb{H}}$. Fix a finite set $I \subset \mathbb{C}^{2}$. For each $n=-1,0, \ldots$ and $J \subset I$, the topological space $\widetilde{\mathfrak{B}}_{J}(n)$ is naturally graded as a product of graded spaces, and given a morphism $f \in \widetilde{\Delta}_{J}(m, n)$, the induced map $\widetilde{\mathfrak{B}}_{J}(n) \rightarrow \widetilde{\mathfrak{B}}_{J}(m)$ preserves the grading. Denote by $\widetilde{\mathfrak{B}}_{J, k}: \widetilde{\Delta}_{J} \rightarrow$ Top the functor obtained by taking the degree $k$ component of $\widetilde{\mathfrak{B}}_{J}$. Let $h_{J}:\left\|\mathfrak{B}_{J}\right\| \rightarrow \overline{\mathfrak{M}}_{J}$ be the map in Definition 5.2. The objective of this section is to prove Theorem 1.3, which we now restate:

Theorem 1.3. If, for every $J \subset I$ with cardinality $\# J \leq k$, the map $h_{J}$ is a homotopy equivalence in degree $k$, then $h_{I}$ is a homotopy equivalence in degree $k$.

Let $\mathfrak{C}$ be as in Definition 3.9 and let $\mathfrak{M}: \mathfrak{C} \rightarrow$ Top be the functor introduced in Proposition 3.10

Definition 6.1. Let $\mathfrak{C}_{I, k}$ be the full subcategory of $\mathfrak{C}$ whose objects are the subsets $J \subset I$ with $\# J \leq k$.

We first need the following result (by nerve of an open cover we mean the functor of Example 2.1):

Lemma 6.2. Let $\mathfrak{M}_{k}: \mathfrak{C} \rightarrow$ Top denote the degree $k$ component of the functor $\mathfrak{M}$ and write $\mathfrak{M}_{J, k}$ for its value $\overline{\text { on } J} \in \mathfrak{C}$. Then the collection $\mathcal{U}=\left\{\pi_{J, I}^{*} \mathfrak{M}_{J, k}\right\}_{J \in \mathfrak{C}_{I, k}}$ is an open cover of $\mathfrak{M}_{I, k}$ and the maps $\pi_{J, I}^{*}$ induce homeomorphisms between the restriction of $\mathfrak{M}_{k}$ to $\mathfrak{C}_{I, k}$ and the nerve of $\mathcal{U}$.

Proof. [42, Theorem 2.1]

We now turn to the proof of Theorem 1.3

Proof. It is enough to show that the map $\left\|\mathfrak{B}_{I, k}\right\| \rightarrow\left\|\widetilde{\mathfrak{B}}_{I, k}\right\|$ is a homotopy equivalence. For each morphism $j: J \rightarrow I$, it will be convenient to replace the functor $\widetilde{\mathfrak{B}}_{J}$ with the functor $\Delta_{j}^{*} \widetilde{\mathfrak{B}}_{J}: \widetilde{\Delta}_{I}^{\text {op }} \rightarrow \underline{\text { Top }}$ (see equation (5.5) $)$. Let $\Delta^{*} \widetilde{\mathfrak{B}}_{k}: \widetilde{\Delta}_{I}^{\text {op }} \times \mathfrak{C}_{I, k} \rightarrow$ Top be the functor defined on objects by

$$
\Delta^{*} \widetilde{\mathfrak{B}}_{k}(n, J)=\Delta_{j}^{*} \widetilde{\mathfrak{B}}_{J, k}(n) ;
$$

given morphisms $i:\left(J_{1}, j_{1}\right) \rightarrow\left(J_{2}, j_{2}\right)$ and $f \in \widetilde{\Delta}_{I}(m, n)$, we define $\Delta^{*} \widetilde{\mathfrak{B}}(f, i)$ by the diagonal map in the following commutative diagram (for the vertical maps in the diagram see equation (5.6)):

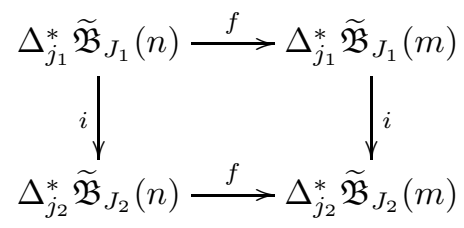


We define the functor $\Delta^{*} \mathfrak{B}_{k}: \Delta_{I}^{\mathrm{op}} \times \mathfrak{C}_{I, k} \rightarrow$ Top by restricting $\Delta^{*} \widetilde{\mathfrak{B}}_{k}$. We claim that the maps

$$
\begin{gathered}
\underset{\mathfrak{C}_{I, k}}{\operatorname{hcolim}}\left(\underset{\Delta_{I}^{\text {op }}}{\operatorname{hcolim}} \Delta^{*} \mathfrak{B}_{k}\right) \rightarrow \underset{\Delta_{I}^{\text {op }}}{\operatorname{hcolim}} \mathfrak{B}_{I, k}, \\
\underset{\mathfrak{C}_{I, k}}{\operatorname{hcolim}}\left(\underset{\widetilde{\Delta}_{I}^{\text {op }}}{\operatorname{hcolim}} \Delta^{*} \widetilde{\mathfrak{B}}_{k}\right) \rightarrow \underset{\widetilde{\Delta}_{I}^{\text {op }}}{\operatorname{hcolim}} \widetilde{\mathfrak{B}}_{I, k}
\end{gathered}
$$

induced by the maps $\Delta_{j}^{*} \widetilde{\mathfrak{B}}_{J, k} \rightarrow \widetilde{\mathfrak{B}}_{I, k}$ are homotopical equivalences; the theorem will follow since we then have a commutative diagram:

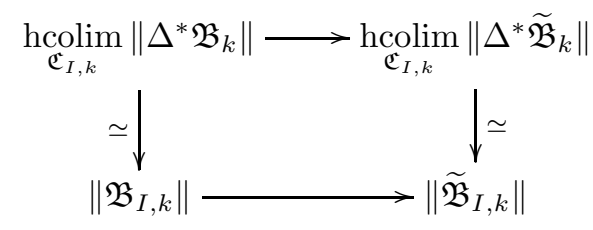

and by hypothesis (and the homotopy invariance of the homotopy colimit), the top horizontal map is a homotopy equivalence. We first prove that the map in equation (6.3) is a homotopy equivalence. From the commutation of homotopy colimits [23, Section 6] we get

$$
\underset{\mathfrak{C}_{I, k}}{\operatorname{hcolim}}\left(\underset{\substack{\Delta_{I}^{\text {op }} \\ \operatorname{hcolim}}}{ } \Delta^{*} \mathfrak{B}_{k}\right) \cong \underset{\Delta_{I}^{\text {op }}}{\operatorname{hcolim}}\left(\underset{\mathfrak{C}_{I, k}}{\operatorname{hcolim}} \Delta^{*} \mathfrak{B}_{k}\right),
$$

so it is enough to show that we have a weak equivalence of $\Delta_{I}^{\mathrm{op}}$-diagrams hcolim $\mathfrak{C}_{I, k} \Delta^{*} \mathfrak{B}_{k} \simeq$ $\mathfrak{B}_{I, k}$ which we now prove. Let $Z_{k} \subset \mathbb{Z} \times \mathbb{Z}^{(n+1)(\# I)}$ be the subset of tuples of non-negative integers whose sum is $k$. We write an element $\mathbf{k} \in Z_{k}$ as $\mathbf{k}=$ $\left(k_{0},\left(k_{\alpha x}\right)_{\substack{x \in I \\ \alpha=0, \ldots, n}}\right)$. Unraveling Definition 5.2 we see that, for $n \geq 0$, we have $\mathfrak{B}_{I, k}(n)=\coprod_{\mathbf{k}} \mathfrak{B}_{I, \mathbf{k}}(n)$ where

$$
\mathfrak{B}_{I, \mathbf{k}}(n)=\mathfrak{M}_{\emptyset, k_{0}} \times\left(\prod_{\substack{\alpha=1, \ldots, n \\ x \in I}} \mathfrak{M}_{\emptyset, k_{\alpha x}}\right) \times\left(\prod_{x \in I} \mathfrak{M}_{x, k_{0 x}}\right) .
$$

Let supp $\mathbf{k} \subset I$ be the set of points such that there is an $\alpha$ for which $k_{\alpha x}>0$. If we let

$$
\mathfrak{B}_{J, \mathbf{k}}(n)= \begin{cases}\mathfrak{B}_{I, \mathbf{k}}(n), & \text { if } \operatorname{supp} \mathbf{k} \subset J ; \\ \emptyset, & \text { if } \operatorname{supp} \mathbf{k} \not \subset J,\end{cases}
$$

then $\mathfrak{B}_{J, k}(n) \cong \coprod_{\mathbf{k}} \mathfrak{B}_{J, \mathbf{k}}(n)$ and under this isomorphism the map $\mathfrak{B}_{J, k}(n) \rightarrow$ $\mathfrak{B}_{I, k}(n)$ corresponds to inclusion. Let $\mathfrak{D}_{I, \mathbf{k}} \subset \mathfrak{C}_{I, k}$ denote the full subcategory whose objects $J$ satisfy supp $\mathbf{k} \subset J$. Then, using the properties of the homotopy colimit (Proposition 2.7) we obtain:

$$
\begin{aligned}
\left(\operatorname{Calim}_{I, k} \Delta^{*} \mathfrak{B}_{k}\right)(n) \cong \coprod_{\mathbf{k} \in Z_{k}} \operatorname{hcolim}_{J \in \mathfrak{C}_{I, \mathbf{k}}} \mathfrak{B}_{J, \mathbf{k}}(n) & \cong \coprod_{\mathbf{k} \in Z_{k}} \underset{J \in \mathfrak{D}_{I, \mathbf{k}}}{\operatorname{hcolim}} \mathfrak{B}_{I, \mathbf{k}}(n) \\
& \cong \coprod_{\mathbf{k} \in Z_{k}} B \mathfrak{D}_{I, \mathbf{k}} \times \mathfrak{B}_{I, \mathbf{k}}(n) \simeq \mathfrak{B}_{I, k}(n)
\end{aligned}
$$

because $\mathfrak{D}_{I, \mathbf{k}}$ has an initial object, namely: supp $\mathbf{k}$. To complete the proof we need to show that the map in equation (6.3 in a homotopy equivalence. We just need to show that $\left(\operatorname{hcolim}_{\mathfrak{C}_{I, k}} \Delta^{*} \widetilde{\mathfrak{B}}_{k}\right)(-1) \simeq \widetilde{\mathfrak{B}}_{I, k}(-1)$ that is (see Definition [5.2), 
$\operatorname{hcolim}_{\mathfrak{C}_{I, k}} \mathfrak{M}_{k} \simeq \mathfrak{M}_{I, k}$. This immediately follows from Lemma 6.2 and 43 , Proposition 4.1].

\section{The GLUing MAPS FOR $k=1,2$}

In this section we define the gluing maps $\boxplus$ used in the statement of Theorem 1.1 The definition is based on the monad description of holomorphic bundles introduced in [14, 26] which we now review.

7.1. The monad description of the moduli spaces. We first set some notation. Let $\left[x_{1}, x_{2}, x_{3}\right] \in \mathbb{P}^{2}$ be homogeneous coordinates, and let $L_{\infty}=\left\{\left[x_{1}, x_{2}, x_{3}\right] \in \mathbb{P}^{2}\right.$ : $\left.x_{3}=0\right\} \subset \mathbb{P}^{2}$ be the line at infinity. We identify the blow up $\tilde{\mathbb{P}}_{\{0\}}^{2}$ of $\mathbb{P}^{2}$ at $[0,0,1]$ with the subvariety of $\mathbb{P}^{2} \times \mathbb{P}^{1}$ consisting of the pairs $\left(\left[x_{1}, x_{2}, x_{3}\right],\left[y_{1}, y_{2}\right]\right)$ such that $x_{1} y_{1}+x_{2} y_{2}=0$.

Let $\mathbb{V}$ be a finite dimensional complex Hermitian vector space and let $W, W_{0}$, $W_{1}$ be complex vector spaces of dimension $k$. Let $\mathcal{R}_{W}^{\mathbb{V}}$ be the space of 4-tuples $\left(a_{1}, a_{2}, b, c\right)$ where $a_{i} \in \operatorname{End}(W), b \in \operatorname{Hom}(\mathbb{V}, W)$ and $c \in \operatorname{Hom}(W, \mathbb{V})$, obeying the integrability condition

$$
a_{1} a_{2}-a_{2} a_{1}+b c=0
$$

and let $\tilde{\mathcal{R}}_{W_{0}, W_{1}}^{\mathbb{V}}$ be the space of 5 -tuples $\left(\tilde{a}_{1}, \tilde{a}_{2}, \tilde{d}, \tilde{b}, \tilde{c}\right)$ where $\tilde{a}_{i} \in \operatorname{Hom}\left(W_{1}, W_{0}\right)$, $\tilde{d} \in \operatorname{Hom}\left(W_{0}, W_{1}\right), \tilde{b} \in \operatorname{Hom}\left(\mathbb{V}, W_{0}\right)$ and $\tilde{c} \in \operatorname{Hom}\left(W_{1}, \mathbb{V}\right)$, such that

$$
\tilde{a}_{1}\left(W_{1}\right)+\tilde{a}_{2}\left(W_{1}\right)+\tilde{b}(\mathbb{V})=W_{0},
$$

obeying the integrability condition

$$
\tilde{a}_{1} \tilde{d} \tilde{a}_{2}-\tilde{a}_{2} \tilde{d} \tilde{a}_{1}+\tilde{b} \tilde{c}=0
$$

Notation 7.4. Except for the proof or Proposition 7.11 below, we always take $W=$ $W_{1}$ and write

$$
\mathcal{R}_{W_{1}}^{\mathbb{V}}=\mathcal{R}_{k}, \quad \tilde{\mathcal{R}}_{W_{0}, W_{1}}^{\mathbb{V}}=\tilde{\mathcal{R}}_{k} \quad\left(k=\operatorname{dim} W_{0}=\operatorname{dim} W_{1}\right) .
$$

Also, we fix for each $k$ an isomorphism $\chi_{k}: W_{0} \rightarrow W_{1}$.

For each $r=\left(a_{1}, a_{2}, b, c\right) \in \mathcal{R}_{k}$ and $x=\left(x_{1}, x_{2}, x_{3}\right) \in \mathbb{C}^{3}$ consider the linear maps $A_{r, x}: W_{1} \rightarrow W_{1}^{2} \oplus \mathbb{V}$ and $B_{r, x}: W_{1}^{2} \oplus \mathbb{V} \rightarrow W_{1}$ given in matrix form by

$$
A_{r, x}=\left(\begin{array}{c}
x_{1}-a_{1} x_{3} \\
x_{2}-a_{2} x_{3} \\
c x_{3}
\end{array}\right), \quad B_{r, x}=\left(\begin{array}{lll}
-x_{2}+a_{2} x_{3} & x_{1}-a_{1} x_{3} & b x_{3}
\end{array}\right) \text {. }
$$

Then the vector spaces $\operatorname{Im} A_{r, x}$ and $\operatorname{Ker} B_{r, x}$ depend only on $\left[x_{1}, x_{2}, x_{3}\right] \in \mathbb{P}^{2}$. The 4-tuple $r$ is called nondegenerate if:

$$
A_{r, x} \text { and } B_{r, x} \text { have maximal rank for every }[x] \in \mathbb{P}^{2} \text {. }
$$

Let $\mathcal{R}_{k}^{\text {reg }} \subset \mathcal{R}_{k}$ denote the subspace of nondegenerate configurations. To each $r \in$ $\mathcal{R}_{k}^{\text {reg }}$ we assign a framed holomorphic bundle $\left(\mathcal{E}_{r}, \phi_{r}\right)$ over $\mathbb{P}^{2}$ as follows: Consider the subbundles $\operatorname{Im} A_{r}, \operatorname{Ker} B_{r}$ of the trivial bundle $\left(W_{1}^{2} \oplus \mathbb{V}\right) \times \mathbb{P}^{2}$. The integrability condition (7.1) is equivalent to $\operatorname{Im} A_{r} \subset \operatorname{Ker} B_{r}$ and we define $\mathcal{E}_{r}$ as the quotient:

$$
\mathcal{E}_{r}=\operatorname{Ker} B_{r} / \operatorname{Im} A_{r} .
$$

The inverse of the trivialization $\phi_{r}$ is the composition

$$
\phi_{r}^{-1}: \mathbb{V} \times L_{\infty} \rightarrow \operatorname{Ker} B_{r} \rightarrow \mathcal{E}_{r}
$$


where the first map is induced, for each $[x] \in L_{\infty}$, by the inclusion $\mathbb{V} \rightarrow \operatorname{Ker} B_{r, x}$.

In a similar way, given $\tilde{r}=\left(\tilde{a}_{1}, \tilde{a}_{2}, \tilde{d}, \tilde{b}, \tilde{c}\right) \in \tilde{\mathcal{R}}_{k}, x=\left(x_{1}, x_{2}, x_{3}\right) \in \mathbb{C}^{3}$ and $y=\left(y_{1}, y_{2}\right) \in \mathbb{C}^{2}$, we have linear maps

$$
\tilde{A}_{\tilde{r}, x}: W_{1} \oplus W_{0} \rightarrow\left(W_{0} \oplus W_{1}\right)^{2} \oplus \mathbb{V} \quad \text { and } \quad \tilde{B}_{\tilde{r}, x}:\left(W_{0} \oplus W_{1}\right)^{2} \oplus \mathbb{V} \rightarrow W_{0} \oplus W_{1}
$$

defined by

$$
\tilde{A}_{\tilde{r}, x}=\left(\begin{array}{cc}
\tilde{a}_{1} x_{3} & -y_{2} \\
x_{1}-\tilde{d} \tilde{a}_{1} x_{3} & 0 \\
\tilde{a}_{2} x_{3} & y_{1} \\
x_{2}-\tilde{d} \tilde{a}_{2} x_{3} & 0 \\
\tilde{c} x_{3} & 0
\end{array}\right), \quad \tilde{B}_{\tilde{r}, x}=\left(\begin{array}{ccccc}
x_{2} & \tilde{a}_{2} x_{3} & -x_{1} & -\tilde{a}_{1} x_{3} & \tilde{b} x_{3} \\
\tilde{d} y_{1} & y_{1} & \tilde{d} y_{2} & y_{2} & 0
\end{array}\right)
$$

The subspace $\tilde{\mathcal{R}}_{k}^{\text {reg }} \subset \tilde{\mathcal{R}}_{k}$ of non-degenerate 5-tuples is the space of elements $\tilde{r} \in$ $\tilde{\mathcal{R}}_{k}^{\text {reg }}$ such that

$$
\tilde{A}_{\tilde{r}, x} \text { and } \tilde{B}_{\tilde{r}, x} \text { have maximal rank for every }([x],[y]) \in \tilde{\mathbb{P}}_{\{0\}}^{2} .
$$

The integrability condition (7.3) is equivalent to $\operatorname{Im} \tilde{A}_{\tilde{r}} \subset \operatorname{Ker} \tilde{B}_{\tilde{r}}$ (provided $x_{1} y_{1}+$ $\left.x_{2} y_{2}=0\right)$ and we define a holomorphic bundle over $\tilde{\mathbb{P}}_{\{0\}}^{2}$ by $\tilde{\mathcal{E}}_{\tilde{r}}=\operatorname{Ker} \tilde{B}_{\tilde{r}} / \operatorname{Im} \tilde{A}_{\tilde{r}}$; as before (see equation (7.7)), the inverse of the trivialization $\tilde{\phi}_{\tilde{r}}$ is induced by the inclusion $\mathbb{V} \rightarrow \operatorname{Ker} \tilde{B}_{\tilde{r}}$.

The groups $G L\left(W_{1}\right)$ and $G L\left(W_{0}\right) \times G L\left(W_{1}\right)$ act on $\mathcal{R}_{k}$ and $\tilde{\mathcal{R}}_{k}$, respectively by

$$
\begin{aligned}
g \cdot\left(a_{1}, a_{2}, b, c\right) & =\left(g a_{1} g^{-1}, g a_{2} g^{-1}, g b, c g^{-1}\right), \quad \text { and } \\
\left(g_{0}, g_{1}\right) \cdot\left(\tilde{a}_{1}, \tilde{a}_{2}, \tilde{d}, \tilde{b}, \tilde{c}\right) & =\left(g_{0} \tilde{a}_{1} g_{1}^{-1}, g_{0} \tilde{a}_{2} g_{1}^{-1}, g_{1} \tilde{d} g_{0}^{-1}, g_{0} \tilde{b}, \tilde{c} g_{1}^{-1}\right) .
\end{aligned}
$$

The group actions preserve the nondegeneracy conditions and we denote the quotients by

$$
M_{\emptyset, k}^{\mathbb{V}}=\mathcal{R}_{k}^{\mathrm{reg}} / G L\left(W_{1}\right) \quad \text { and } \quad M_{\{0\}, k}^{\mathbb{V}}=\tilde{\mathcal{R}}_{k}^{\mathrm{reg}} /\left(G L\left(W_{0}\right) \times G L\left(W_{1}\right)\right) .
$$

Theorem (Donaldson, King). The actions of $G L\left(W_{1}\right)$ on $\mathcal{R}_{k}^{\text {reg }}$ and $G L\left(W_{0}\right) \times$ $G L\left(W_{1}\right)$ on $\tilde{\mathcal{R}}_{k}^{\text {reg }}$ are free and the assignments $r \mapsto\left(\mathcal{E}_{r}, \phi_{r}\right)$ and $\tilde{r} \mapsto\left(\tilde{\mathcal{E}}_{\tilde{r}}, \tilde{\phi}_{\tilde{r}}\right)$ descend to the quotient defining isomorphisms

$$
\psi_{\emptyset}: M_{\emptyset, k}^{\mathbb{V}} \stackrel{\cong}{\rightarrow} \mathfrak{M}_{\emptyset, k}^{\mathbb{V}}, \quad \psi_{\{0\}}: M_{\{0\}, k}^{\mathbb{V}} \stackrel{\cong}{\rightarrow} \mathfrak{M}_{\{0\}, k}^{\mathbb{V}} .
$$

Proof. The statement for $\mathbb{P}^{2}$ is proven in [14, Proposition 1]. The statement for $\tilde{\mathbb{P}}_{\{0\}}^{2}$ is proven in [26, Theorem 3.4.1].

Proposition 7.11. Let $\mathbb{V}_{1}, \mathbb{V}_{2}$ be finite dimensional complex Hermitian vector spaces and let $J \subset \mathbb{C}^{2}$ be either $\emptyset$ or $\{0\}$.

(1) Given a linear isometry $\alpha: \mathbb{V}_{1} \rightarrow \mathbb{V}_{2}$, with dual $\alpha^{*}: \mathbb{V}_{2} \rightarrow \mathbb{V}_{1}$, the isomorphisms in equation (7.10) take the induced maps $\mathfrak{M}_{J, k}^{\mathbb{V}_{1}} \rightarrow \mathfrak{M}_{J, k}^{\mathbb{V}_{2}}$ to the maps $\alpha: M_{J, k}^{\mathbb{V}_{1}} \rightarrow M_{J, k}^{\mathbb{V}_{2}}$ given by

$$
\begin{array}{rlrl}
{\left[a_{1}, a_{2}, b, c\right]} & \mapsto\left[a_{1}, a_{2}, b \circ \alpha^{*}, \alpha \circ c\right] & & (J=\emptyset) \\
{\left[\tilde{a}_{1}, \tilde{a}_{2}, \tilde{d}, \tilde{b}, \tilde{c}\right]} & \mapsto\left[\tilde{a}_{1}, \tilde{a}_{2}, \tilde{d}, \tilde{b} \circ \alpha^{*}, \alpha \circ \tilde{c}\right] & (J=\{0\})
\end{array}
$$


(2) The isomorphisms in equation (7.10) take Whitney sum $\mathfrak{M}_{J, k_{1}}^{\mathbb{V}_{1}} \times \mathfrak{M}_{J, k_{2}}^{\mathbb{V}_{2}} \rightarrow$ $\mathfrak{M}_{J, k_{1}+k_{2}}^{\mathbb{V}_{1} \oplus \mathbb{V}_{2}}$ to the maps $\omega: M_{J, k_{1}}^{\mathbb{V}_{1}} \times M_{J, k_{2}}^{\mathbb{V}_{2}} \rightarrow M_{J, k_{1}+k_{2}}^{\mathbb{V}_{1} \oplus \mathbb{V}_{2}}$ induced by direct sum: $\left(\left[a_{1}, a_{2}, b, c\right],\left[a_{1}^{\prime}, a_{2}^{\prime}, b^{\prime}, c^{\prime}\right]\right) \mapsto\left[a_{1} \oplus a_{1}^{\prime}, a_{2} \oplus a_{2}^{\prime}, b \oplus b^{\prime}, c \oplus c^{\prime}\right] \quad J=\emptyset$ $\left(\left[\tilde{a}_{1}, \tilde{a}_{2}, \tilde{d}, \tilde{b}, \tilde{c}\right],\left[\tilde{a}_{1}^{\prime}, \tilde{a}_{2}^{\prime}, \tilde{d}^{\prime}, \tilde{b}^{\prime}, \tilde{c}^{\prime}\right]\right) \mapsto\left[\tilde{a}_{1} \oplus \tilde{a}_{1}^{\prime}, \tilde{a}_{2} \oplus \tilde{a}_{2}^{\prime}, \tilde{d} \oplus \tilde{d}^{\prime}, \tilde{b} \oplus \tilde{b}^{\prime}, \tilde{c} \oplus \tilde{c}^{\prime}\right] \quad J=\{0\}$

(3) For each $z=\left(z_{1}, z_{2}\right) \in \mathbb{C}^{2}$, let $\tau_{z}: \mathbb{P}^{2} \rightarrow \mathbb{P}^{2}$ be the translation map defined by

$$
\tau_{z}\left(\left[x_{1}, x_{2}, x_{3}\right]\right)=\left[x_{1}+z_{1} x_{3}, x_{2}+z_{2} x_{3}, x_{3}\right] .
$$

Then the isomorphisms in equation (7.10) take the pullback map $\tau_{z}^{*}: \mathfrak{M}_{\emptyset, k}^{\mathbb{V}} \rightarrow$ $\mathfrak{M}_{\emptyset, k}^{\mathbb{V}}$ to the map $\tau_{z}^{*}: M_{\emptyset, k}^{\mathbb{V}} \rightarrow M_{\emptyset, k}^{\mathbb{V}}$ given by

$$
\tau_{z}^{*}\left(\left[a_{1}, a_{2}, b, c\right]\right)=\left[a_{1}-z_{1}, a_{2}-z_{2}, b, c\right] .
$$

(4) Let $\pi: \tilde{\mathbb{P}}_{\{0\}}^{2} \rightarrow \mathbb{P}^{2}$ be the blowup map. The pullback map $\pi^{*}: \mathfrak{M}_{\emptyset, k}^{\mathbb{V}} \rightarrow \mathfrak{M}_{\{0\}, k}^{\mathbb{V}}$ is taken by the isomorphisms in equation (7.10) to the map $\pi^{*}: M_{\emptyset, k}^{\mathbb{V}} \rightarrow$ $M_{\{0\}, k}^{\mathbb{V}}$ given as follows: fix a vector space isomorphism $\chi_{k}: W_{0} \rightarrow W_{1}$; then

$$
\pi^{*}\left(\left[a_{1}, a_{2}, b, c\right]\right)=\left[\chi_{k}^{-1} a_{1}, \chi_{k}^{-1} a_{2}, \chi_{k}, \chi_{k}^{-1} b, c\right] .
$$

Proof. We prove (1) and (2) only for $J=\emptyset$ since the proof for $J=\{0\}$ is completely analogous. We'll use the notation $\mathcal{R}_{W}^{\mathbb{V}}$ instead of $\mathcal{R}_{k}$ (see Notation 7.4).

(1) We assume first that $\operatorname{dim} \mathbb{V}_{1}=\operatorname{dim} \mathbb{V}_{2}$. Given $r=\left(a_{1}, a_{2}, b, c\right) \in \mathcal{R}_{W}^{\mathbb{V}_{1}}$ let $\alpha(r)=\left(a_{1}, a_{2}, b \circ \alpha^{*}, \alpha \circ c\right) \in \mathcal{R}_{W}^{\mathbb{V}_{2}}$. We want to show that $\left[\mathcal{E}_{\alpha(r)}, \phi_{\alpha(r)}\right]=$ $\left[\mathcal{E}_{r},(\alpha \times \mathrm{id}) \circ \phi_{r}\right]$ (see Definition 3.4(2)). We have a commutative diagram:

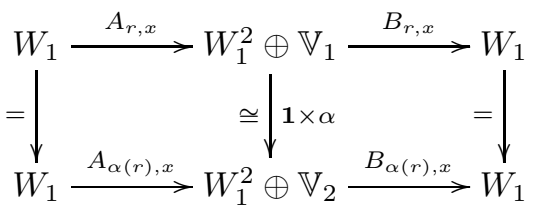

which induces an isomorphism $\psi_{\alpha}: \mathcal{E}_{r} \rightarrow \mathcal{E}_{\alpha(r)}$ and restricting to $L_{\infty}$ we have $(\alpha \times \mathrm{id}) \circ \phi_{r}=\phi_{\alpha(r)} \circ \psi_{\alpha}$ (see equation (7.7)) so

$$
\left[\mathcal{E}_{r},(\alpha \times \mathrm{id}) \circ \phi_{r}\right]=\left[\mathcal{E}_{r}, \phi_{\alpha(r)} \circ \psi_{\alpha}\right]=\left[\mathcal{E}_{\alpha(r)}, \phi_{\alpha(r)}\right] .
$$

Now consider the general case. Any isometry $\alpha: \mathbb{V}_{1} \rightarrow \mathbb{V}_{2}$ is the composition of an isomorphism $\mathbb{V}_{1} \rightarrow \alpha\left(\mathbb{V}_{1}\right)$ and an inclusion $\alpha\left(\mathbb{V}_{1}\right) \rightarrow \alpha\left(\mathbb{V}_{1}\right) \oplus$ $\alpha\left(\mathbb{V}_{1}\right)^{\perp}=\mathbb{V}_{2}$ whose induced map $M_{J, k}^{\alpha\left(\mathbb{V}_{1}\right)} \rightarrow \mathfrak{M}_{J, k}^{\mathbb{V}_{2}}$ is given by direct sum with a trivial bundle so the result follows from (2).

(2) Given $r \in \mathcal{R}_{W}^{\mathbb{V}}$ and $r^{\prime} \in \mathcal{R}_{W^{\prime}}^{\mathbb{V}^{\prime}}$ let $r \oplus r^{\prime} \in \mathcal{R}_{W \oplus W^{\prime}}^{\mathbb{V} \oplus \mathbb{V}^{\prime}}$ be the 4-tuple obtained by direct sum in each coordinate. Then from the definition of the linear maps $A$ and $B$ (equation (7.5)) it is straightforward to see that the complex

$$
W \oplus W^{\prime} \stackrel{A_{r \oplus r^{\prime}, x}}{\longrightarrow}\left(W \oplus W^{\prime}\right)^{2} \oplus\left(\mathbb{V} \oplus \mathbb{V}^{\prime}\right) \stackrel{B_{r \oplus r^{\prime}}}{\longrightarrow} W \oplus W^{\prime}
$$

gives rise to an isomorphism $\mathcal{E}_{r \oplus r^{\prime}} \cong \mathcal{E}_{r} \oplus \mathcal{E}_{r^{\prime}}$.

(3) For each $x \in \mathbb{P}^{2}$ let $\tau_{z} x$ be as in equation (7.12). The map $\tau_{z}^{*}: \mathcal{R}_{k} \rightarrow \mathcal{R}_{k}$ defined by $\tau_{z}^{*}\left(a_{1}, a_{2}, b, c\right)=\left(a_{1}-z_{1}, a_{2}-z_{2}, b, c\right)$ preserves the nondegeneracy conditions and the framing and from equation (7.5) we immediately get $A_{r, \tau_{z} x}=A_{\tau_{z}^{*} r, x}$ and $B_{r, \tau_{z} x}=B_{\tau_{z}^{*} r, x}$. It follows that $\tau_{z}^{*} \mathcal{E}_{r}=\mathcal{E}_{\tau_{z}^{*} r}$. 
(4) In 9, Lemma 4.1] it was shown that, if we take $W=W_{0}$ (see Notation 7.4) then pullback is given by the map $j\left(a_{1}, a_{2}, b, c\right)=\left(a_{1} \chi_{k}^{-1}, a_{2} \chi_{k}^{-1}, \chi_{k}, b, c \chi_{k}^{-1}\right)$. In this paper we take $W=W_{1}$. If we let $f: M_{\emptyset, k}^{\mathbb{V}}=\mathcal{R}_{W_{1}}^{\mathbb{V}} / G L\left(W_{1}\right) \rightarrow$ $\mathcal{R}_{W_{0}}^{\mathbb{V}} / G L\left(W_{0}\right)$ be the isomorphism defined by

$$
f\left(a_{1}, a_{2}, b, c\right)=\left(\chi_{k}^{-1} a_{1} \chi_{k}, \chi_{k}^{-1} a_{2} \chi_{k}, \chi_{k}^{-1} b, c \chi_{k}\right)
$$

then $j \circ f\left(a_{1}, a_{2}, b, c\right)=\left(\chi_{k}^{-1} a_{1}, \chi_{k}^{-1} a_{2}, \chi_{k}, \chi_{k}^{-1} b, c\right)$, which finishes the proof.

7.2. Completion of the moduli space. From now on (except in Proposition 7.46) we work with a fixed finite dimensional complex Hermitian vector space $\mathbb{V}$, which we will omit from the notation.

We now drop the nondegeneracy conditions (7.6) and (7.8), and consider the geometric invariant theory quotients, formed by identifying orbits whose closures intersect [27, Remark 8.14]:

$$
\bar{M}_{\emptyset, k}=\mathcal{R}_{k} / / G L\left(W_{1}\right), \quad \bar{M}_{\{0\}, k}=\tilde{\mathcal{R}}_{k} / /\left(G L\left(W_{0}\right) \times G L\left(W_{1}\right)\right) .
$$

Theorem (King). The spaces $\bar{M}_{\emptyset, k}$ and $\bar{M}_{\{0\}, k}$ are isomorphic to the DonaldsonUhlenbeck completions $\overline{\mathfrak{M}}_{\emptyset, k}$ and $\overline{\mathfrak{M}}_{\{0\}, k}$.

Proof. The statement for $\mathbb{P}^{2}$ is proven in [26, Theorem 5.2.7]. The statement for $\tilde{\mathbb{P}}_{\{0\}}^{2}$ is proven in [26, Theorem 5.3.7].

We briefly sketch how the isomorphisms $\psi_{\emptyset}, \psi_{\{0\}}$ in equation (7.10) extend to the completion. For more details see [26, section 5]. The Donaldson-Uhlenbeck completion $\overline{\mathfrak{M}}_{\emptyset, k}$ consists of pairs $([\mathcal{E}, \phi], \ell)$ where $[\mathcal{E}, \phi] \in \mathfrak{M}_{\emptyset, j}$ for some $j \leq k$, and $\ell: \mathbb{C}^{2} \rightarrow \mathbb{Z}$ is a non-negative function which vanishes except at a finite number of points $p_{1}, \ldots, p_{r}$ and is such that $\sum_{i} \ell\left(p_{i}\right)=k-j$. Such a function $\ell$ determines an unordered $(k-j)$-tuple in $\mathbb{C}^{2}$, with each point $p_{i}$ appearing with multiplicity $\ell\left(p_{i}\right)$, which in turn correspond to a point in the symmetric product $S^{k-j} \mathbb{C}^{2}$. So the Donaldson-Uhlenbeck completion has a natural stratification

$$
\overline{\mathfrak{M}}_{\emptyset, k}=\bigcup_{j=0}^{k} \mathfrak{M}_{\emptyset, j} \times S^{k-j} \mathbb{C}^{2} .
$$

Any 4-tuple $r \in \mathcal{R}_{k}$ is equivalent to a direct sum $r^{\text {reg }} \oplus r^{\Delta}$ where $r^{\text {reg }} \in \mathcal{R}_{j}^{\text {reg }}$ for some $0 \leq j \leq k$, determining a point in $\mathfrak{M}_{\emptyset, j}$, and $r^{\Delta}=\left(a_{1}^{\Delta}, a_{2}^{\Delta}, 0,0\right)$ where $a_{1}^{\Delta}$, $a_{2}^{\Delta}$ are $(k-j) \times(k-j)$ diagonal matrices. For each simultaneous eigenvector, the eigenvalues of $a_{1}, a_{2}$ determine a point $p \in \mathbb{C}^{2}$. The $k-j$ points thus obtained define an unordered $(k-j)$-tuple in $S^{k-j} \mathbb{C}^{2}$.

Remark 7.14. If $r=r^{\mathrm{reg}} \oplus r^{\Delta}$ is a degenerate configuration then $\mathcal{E}_{r}=\operatorname{Ker} B_{r} / \operatorname{Im} A_{r}$ is a coherent sheaf which is locally free except at a finite number of points. The holomorphic bundle $\mathcal{E}_{r^{\text {reg }}}$ is the double dual of the sheaf $\mathcal{E}_{r}$ and $\ell$ is the cycle associated to the zero-dimensional sheaf $\mathcal{E}_{\text {reg }} / \mathcal{E}_{r}$ : at each point $p_{i}$ where $\mathcal{E}_{r}$ is not locally free, $\ell\left(p_{i}\right)=\operatorname{dim}_{\mathbb{C}}\left(\mathcal{E}_{r_{\text {reg }}} / \mathcal{E}_{r}\right)_{p_{i}}$.

The completion $\overline{\mathfrak{M}}_{\{0\}, k}$ can be described in an analogous way to $\overline{\mathfrak{M}}_{\emptyset, k}$ : it is the space of pairs $\left([\mathcal{E}, \phi],\left(p_{1}, \ldots, p_{k-j}\right)\right)$ with $[\mathcal{E}, \phi] \in \mathfrak{M}_{\{0\}, j}$ and $\left(p_{1}, \ldots, p_{k-j}\right)$ an unordered $(k-j)$-tuple in the blowup $\widetilde{\mathbb{C}}^{2}$ of $\mathbb{C}^{2}$ at the origin, which we identify with 
the pairs of points $\left(\left(\lambda_{1}, \lambda_{2}\right),\left[\mu_{1}, \mu_{2}\right]\right) \in \mathbb{C}^{2} \times \mathbb{P}^{1}$ satisfying $\mu_{1} \lambda_{1}+\mu_{2} \lambda_{2}=0$. Any 5 -tuple $\tilde{r} \in \tilde{\mathcal{R}}_{k}$ is equivalent to a direct sum $\tilde{r}^{\text {reg }} \oplus \tilde{r}^{\Delta}$ with $\tilde{r}^{\text {reg }} \in \tilde{\mathcal{R}}_{j}^{\text {reg }}$ for some $j$, determining a point in $\mathfrak{M}_{\{0\}, j}$, and $\tilde{r}^{\Delta}=\left(\tilde{a}_{1}^{\Delta}, \tilde{a}_{2}^{\Delta}, \tilde{d}^{\Delta}, 0,0\right)$ where $\tilde{a}_{1}^{\Delta}, \tilde{a}_{2}^{\Delta}, \tilde{d}^{\Delta}$ are $(k-j) \times(k-j)$ diagonal matrices. For each simultaneous eigenvector $v$ of $\tilde{d}^{\Delta} \tilde{a}_{1}^{\Delta}$ and $\tilde{d}^{\Delta} \tilde{a}_{2}^{\Delta}$, there is a unique $\left[\mu_{1}, \mu_{2}\right] \in \mathbb{P}^{1}$ such that $\mu_{1} \tilde{a}_{1}^{\Delta} v+\mu_{2} \tilde{a}_{2}^{\Delta} v=0$ (this follows from condition (7.2) ) and the eigenvalues $\lambda_{i}$ of $\tilde{d}^{\Delta} \tilde{a}_{i}^{\Delta}$ satisfy $\mu_{1} \lambda_{1}+\mu_{2} \lambda_{2}=0$, determining a point $\left(\left(\lambda_{1}, \lambda_{2}\right),\left[\mu_{1}, \mu_{2}\right]\right) \in \widetilde{\mathbb{C}}^{2}$. The $k-j$ points thus obtained define an unordered $(k-j)$-tuple in $S^{k-j} \widetilde{\mathbb{C}}^{2}$.

7.3. Blowup. Let $\pi: \tilde{X} \rightarrow X$ be the blowup of a complex surface $X$ at a point $z \in X$ and let $L=\pi^{-1}(z)$ be the exceptional divisor. A holomorphic bundle on $\tilde{X}$ determines a holomorphic bundle on $X$ :

Proposition 7.15. Given a holomorphic bundle $\tilde{\mathcal{E}}$ over $\tilde{X}$ there is a unique (up to isomorphism) holomorphic bundle $\mathcal{E}$ over $X$ such that $\left.\left.\left(\pi^{*} \mathcal{E}\right)\right|_{X \backslash L} \cong \tilde{\mathcal{E}}\right|_{\tilde{X} \backslash L}$.

Proof. Uniqueness follows from Hartogs' Theorem: any bundle isomorphism over $X \backslash\{z\}$ can be extended to an isomorphism over $X$. To show existence we take $\mathcal{E}$ to be the double dual of the direct image sheaf: $\mathcal{E}=\left(\pi_{*} \tilde{\mathcal{E}}\right)^{\vee \vee}$. This sheaf is locally free [19. Chapter 2, Proposition 25] and hence it is a holomorphic bundle. Moreover, we have $\left.\left.\left(\pi_{*} \tilde{\mathcal{E}}\right)^{\vee \vee}\right|_{X \backslash\{z\}} \cong \tilde{\mathcal{E}}\right|_{\tilde{X} \backslash L}$ as required.

We represent the double dual of the direct image sheaf by

$$
\pi_{*}^{\vee \vee} \tilde{\mathcal{E}}=\left(\pi_{*} \tilde{\mathcal{E}}\right)^{\vee \vee}
$$

Proposition 7.16. For any holomorphic bundle $\mathcal{E}$ over $X$ we have $\mathcal{E} \cong \pi_{*} \pi^{*} \mathcal{E} \cong$ $\pi_{*}^{\vee \vee} \pi^{*} \mathcal{E}$

Proof. From [11, Lemma 2.2(a)] it follows that $\pi_{*} \pi^{*} \mathcal{E}$ is locally free so $\pi_{*} \pi^{*} \mathcal{E} \cong$ $\pi_{*}^{\vee \vee} \pi^{*} \mathcal{E}$. Since $\left.\pi\right|_{\tilde{X} \backslash L}$ is an isomorphism we have $\left.\left.\mathcal{E}\right|_{X \backslash\{z\}} \cong \pi_{*} \pi^{*} \mathcal{E}\right|_{X \backslash\{z\}}$ and hence, by uniqueness (Proposition 7.15), we get $\mathcal{E} \cong \pi_{*} \pi^{*} \mathcal{E}$.

Now fix a finite set $J \subset \mathbb{C}^{2}$ and a point $x \notin J$, let $I=J \cup\{x\}$ and let $\pi: \tilde{\mathbb{P}}_{I}^{2} \rightarrow \tilde{\mathbb{P}}_{J}^{2}$ be the blowup at $x$. We define a map $\pi_{\bullet}: \mathfrak{M}_{I, k} \rightarrow \overline{\mathfrak{M}}_{J, k}$ as follows: Let $\tilde{\mathcal{E}}$ be a holomorphic bundle over $\tilde{\mathbb{P}}_{I}^{2}$; since $\left.\pi\right|_{L_{\infty}}$ is an isomorphism, a trivialization $\tilde{\phi}$ of $\left.\tilde{\mathcal{E}}\right|_{L_{\infty}}$ induces a trivialization of $\left.\left(\pi_{*}^{\vee \vee} \tilde{\mathcal{E}}\right)\right|_{L_{\infty}}$, which we represent by $\pi_{*}(\tilde{\phi})$; we define

$$
\pi_{\bullet}[\tilde{\mathcal{E}}, \tilde{\phi}]=\left(\left[\pi_{*}^{\vee \vee} \tilde{\mathcal{E}}, \pi_{*}(\tilde{\phi})\right], \ell\right)
$$

where $\ell(p)=0$ for $p \neq x$ and $\ell(x)=c_{2}(\tilde{\mathcal{E}})-c_{2}\left(\pi_{*}^{\vee \vee} \tilde{\mathcal{E}}\right) \geq 0$ (see 11, Equation (2.10)]). From Proposition 7.16 it immediately follows that

$$
\pi_{\bullet} \pi^{*}[\mathcal{E}, \phi]=[\mathcal{E}, \phi] .
$$

Remark 7.19. The map $\pi_{\bullet}$ sends a holomorphic bundle $\tilde{\mathcal{E}}$ to the point in the Donaldson-Uhlenbeck compactification determined by the direct image sheaf $\pi_{*} \tilde{\mathcal{E}}$ : see Remark 7.14

Proposition 7.20. Let $[\tilde{\mathcal{E}}, \tilde{\phi}] \in \mathfrak{M}_{I, k}$. The following are equivalent:

(1) $\left.\tilde{\mathcal{E}}\right|_{L}$ is the trivial bundle.

(2) $\pi_{\bullet}([\tilde{\mathcal{E}}, \tilde{\phi}]) \in \mathfrak{M}_{J, k}$.

(3) $\tilde{\mathcal{E}}=\pi^{*}\left(\pi_{*}^{\vee \vee \tilde{\mathcal{E}}}\right)$. 
Proof. Statement (2) is equivalent to $c_{2}(\tilde{\mathcal{E}})=c_{2}\left(\pi_{*}^{\vee \vee} \tilde{\mathcal{E}}\right)$ so it is clear that $(3) \Rightarrow(2)$ and from [11, Equation (2.10)] it follows that $(2) \Rightarrow(1)$. Finally, from [11, Lemma $2.2(\mathrm{a})(\mathrm{c})]$, it follows that $(1) \Rightarrow(3)$.

Corollary 7.21. Let $I=\{x, y\} \subset \mathbb{C}^{2}$ and let $\mathcal{E}$ be a holomorphic bundle over $\tilde{\mathbb{P}}_{\{y\}}^{2}$. Then, with notation as in Definition 3.4(4), we have

$$
\left(\pi_{x, I}\right)_{*}^{\vee \vee} \circ \pi_{y, I}^{*} \mathcal{E} \cong \pi_{\emptyset, x}^{*} \circ\left(\pi_{\emptyset, y}\right)_{*}^{\vee \vee} \mathcal{E}
$$

Proof. From Proposition 7.15 it follows that, given any holomorphic bundle $\tilde{\mathcal{E}}$ over $\tilde{\mathbb{P}}_{I}^{2}$, we have

$$
\left(\pi_{\emptyset, y}\right)_{*}^{\vee \vee} \circ\left(\pi_{y, I}\right)_{*}^{\vee \vee} \tilde{\mathcal{E}} \cong\left(\pi_{\emptyset, I}\right)_{*}^{\vee \vee} \tilde{\mathcal{E}} \cong\left(\pi_{\emptyset, x}\right)_{*}^{\vee \vee} \circ\left(\pi_{x, I}\right)_{*}^{\vee \vee} \tilde{\mathcal{E}}
$$

Since the bundle $\left(\pi_{x, I}\right)_{*}^{\vee \vee} \circ \pi_{y, I}^{*} \mathcal{E}$ is trivial on the exceptional divisor we get

$$
\begin{aligned}
\left(\pi_{x, I}\right)_{*}^{\vee \vee} \circ \pi_{y, I}^{*} \mathcal{E} & \cong \pi_{\emptyset, x}^{*} \circ\left(\pi_{\emptyset, x}\right)_{*}^{\vee \vee} \circ\left(\pi_{x, I}\right)_{*}^{\vee \vee} \circ \pi_{y, I}^{*} \mathcal{E} & & \text { (Proposition 7.20) } \\
& \cong \pi_{\emptyset, x}^{*} \circ\left(\pi_{\emptyset, y}\right)_{*}^{\vee \vee} \circ\left(\pi_{y, I}\right)_{*}^{\vee \vee} \circ \pi_{y, I}^{*} \mathcal{E} & & \\
& \cong \pi_{\emptyset, x}^{*} \circ\left(\pi_{\emptyset, y}\right)_{*}^{\vee \vee} \mathcal{E} & & \text { (Proposition 7.16) }
\end{aligned}
$$

Proposition 7.22. Let $\pi_{\bullet}: M_{\{0\}, k} \rightarrow \bar{M}_{\emptyset, k}$ be the map induced by the map in equation (7.17) and by the isomorphisms in equation (7.10). Then we have

$$
\pi_{\bullet}\left[a_{1}, a_{2}, d, b, c\right]=\left[d a_{1}, d a_{2}, d b, c\right] .
$$

Proof. For any $\tilde{r} \in \tilde{\mathcal{R}}_{k}^{\text {reg }}, \pi_{\bullet} \tilde{r}$ is nondegenerate away from the blowup point and $\pi^{*} \mathcal{E}_{\pi_{\bullet} \tilde{r}}$ restricted to $\tilde{\mathbb{P}}_{\{0\}}^{2} \backslash L$ is isomorphic to $\tilde{\mathcal{E}}_{\tilde{r}}$ restricted to $\tilde{\mathbb{P}}_{\{0\}}^{2} \backslash L$ (see [26], Proposition 6.1.1 and subsequent discussion). By Proposition 7.15, this implies that $\mathcal{E}_{\pi_{\bullet} \tilde{r}}$ is isomorphic to $\pi_{*}^{\vee \vee} \tilde{\mathcal{E}}$, which finishes the proof.

Definition 7.23. Let $S_{0} M_{\{0\}, 1}=\left\{[\tilde{r}] \in M_{\{0\}, 1}: \pi_{*}^{\vee \vee} \tilde{\mathcal{E}}_{\tilde{r}}\right.$ is the trivial bundle $\}$.

Proposition 7.24. Let $\tilde{r} \in \tilde{\mathcal{R}}_{1}^{\text {reg }}$. The following are equivalent:

(1) $[\tilde{r}] \in S_{0} M_{\{0\}, 1}$.

(2) $\pi_{\bullet}[\tilde{r}]=0 \in \mathbb{C}^{2}=\bar{M}_{\emptyset, 1} \backslash M_{\emptyset, 1}$.

(3) $[\tilde{r}] \notin \pi^{*} M_{\emptyset, 1}$.

(4) $\tilde{r}=\left(a_{1}, a_{2}, 0, b, c\right)$.

Proof. The equivalence (1) $\Leftrightarrow(2)$ follows immediately from the definition of $S_{0} M_{\{0\}, 1}$ and the definition of $\pi_{\bullet}$. To show that (1) $\Leftrightarrow(3)$ note that $[\tilde{r}] \notin S_{0} M_{\{0\}, 1}$ is equivalent to $\pi_{\bullet} \tilde{\mathcal{E}}_{\tilde{r}}=\pi_{*}^{\vee \vee} \tilde{\mathcal{E}}_{\tilde{r}} \in \mathfrak{M}_{\emptyset, 1}$. From Proposition 7.16 it follows that $\tilde{\mathcal{E}} \in \pi^{*} \mathfrak{M}_{\emptyset, 1} \Rightarrow \pi_{*}^{\vee \vee} \tilde{\mathcal{E}} \in \mathfrak{M}_{\emptyset, 1}$ so (1) $\Rightarrow$ (3); from Proposition 7.20 it follows that $\pi_{\bullet} \tilde{\mathcal{E}} \in \mathfrak{M}_{\emptyset, 1} \Rightarrow \tilde{\mathcal{E}} \in \pi^{*} \mathfrak{M}_{\emptyset, 1}$ so $(3) \Rightarrow(1)$. Now let $\tilde{r}=\left(a_{1}, a_{2}, d, b, c\right)$. Then $[\tilde{r}] \in \pi^{*} M_{\emptyset, 1}$ is equivalent to $d$ being an isomorphism (see the beginning of section 3.6 in $[26]$ ) which shows that $(3) \Leftrightarrow(4)$.

7.4. Blowups at points $\boldsymbol{z} \neq \mathbf{0}$. We identify the blow up $\tilde{\mathbb{P}}_{\{z\}}^{2}$ of $\mathbb{P}^{2}$ at a point $z=$ $\left(z_{1}, z_{2}\right) \in \mathbb{C}^{2}$ with the subvariety of $\mathbb{P}^{2} \times \mathbb{P}^{1}$ consisting of the pairs $\left(\left[x_{1}, x_{2}, x_{3}\right],\left[y_{1}, y_{2}\right]\right)$ such that $\left(x_{1}-z_{1}\right) y_{1}+\left(x_{2}-z_{2}\right) y_{2}=0$. Translation in $\mathbb{C}^{2}$ induces an action $\tau: \mathbb{C}^{2} \times \mathbb{P}^{2} \rightarrow \mathbb{P}^{2}$ and an isomorphism $\tau_{z}: \tilde{\mathbb{P}}_{\{0\}}^{2} \rightarrow \tilde{\mathbb{P}}_{\{z\}}^{2}$ given respectively by

$$
\begin{aligned}
\tau_{z}\left(\left[x_{1}, x_{2}, x_{3}\right]\right) & =\left[x_{1}+z_{1} x_{3}, x_{2}+z_{2} x_{3}, x_{3}\right] \quad \text { and } \\
\tau_{z}\left(\left[x_{1}, x_{2}, x_{3}\right],\left[y_{1}, y_{2}\right]\right) & =\left(\left[x_{1}+z_{1} x_{3}, x_{2}+z_{2} x_{3}, x_{3}\right],\left[y_{1}, y_{2}\right]\right) .
\end{aligned}
$$


Let $\psi_{\{0\}}: M_{\{0\}, k} \rightarrow \mathfrak{M}_{\{0\}, k}$ be as in equation (17.10). The translation map $\tau_{z}$ allows us to identify, for any $z \in \mathbb{C}^{2}$, the moduli space $\mathfrak{M}_{\{z\}, k}$ with $M_{\{0\}, k}$, through the isomorphism

$$
\psi_{\{z\}}=\left(\tau_{z}^{-1}\right)^{*} \circ \psi_{\{0\}}: M_{\{0\}, k} \rightarrow \mathfrak{M}_{\{z\}, k} .
$$

Let $\pi_{z}: \tilde{\mathbb{P}}_{z}^{2} \rightarrow \mathbb{P}^{2}$ be the blowup at $z \in \mathbb{C}^{2}$ and let $\pi=\pi_{0}$ be the blowup at 0 . Then

$$
\pi_{z}^{*} \circ \tau_{-z}^{*}=\left(\tau_{z}^{-1}\right)^{*} \circ \pi^{*} \quad \text { and } \quad \pi_{z *}^{\vee \vee} \circ\left(\tau_{z}^{-1}\right)^{*}=\tau_{-z}^{*} \circ \pi_{*}^{\vee \vee} .
$$

7.5. The monad description of the gluing maps. For $k=1$ we have $\operatorname{dim} W_{0}=$ $\operatorname{dim} W_{1}=1$ so there is a canonical isomorphism $\operatorname{End}\left(W_{1}\right) \cong \mathbb{C}$. Consider the maps $f: \bar{M}_{\emptyset, 1} \rightarrow \mathbb{C}$ and $\tilde{f}: \bar{M}_{\{0\}, 1} \rightarrow \mathbb{C}$ defined by

$$
f\left(\left[a_{1}, a_{2}, b, c\right]\right)=a_{1} \quad \text { and } \quad \tilde{f}\left(\left[a_{1}, a_{2}, d, b, c\right]\right)=d a_{1} .
$$

It follows from Proposition $7.11(4)$ that $\tilde{f} \circ \pi^{*}=f$.

Definition 7.27. Let $D=\left\{\left(m, m^{\prime}\right) \in \bar{M}_{\emptyset, 1} \times \bar{M}_{\emptyset, 1}: f(m) \neq f\left(m^{\prime}\right)\right\}$. We define $\boxplus: D \rightarrow \bar{M}_{\emptyset, 2}$ by

$$
\begin{aligned}
{\left[a_{1}, a_{2}, b, c\right] \boxplus\left[a_{1}^{\prime}, a_{2}^{\prime}, b^{\prime}, c^{\prime}\right] } & \\
= & {\left[\left(\begin{array}{cc}
a_{1} & 0 \\
0 & a_{1}^{\prime}
\end{array}\right),\left(\begin{array}{cc}
a_{2} & \frac{b c^{\prime}}{a_{1}^{\prime}-a_{1}} \\
\frac{b^{\prime} c}{a_{1}-a_{1}^{\prime}} & a_{2}^{\prime}
\end{array}\right),\left(\begin{array}{c}
b \\
b^{\prime}
\end{array}\right),\left(\begin{array}{ll}
c & c^{\prime}
\end{array}\right)\right] . }
\end{aligned}
$$

Let

$$
\begin{aligned}
\bar{M}_{\emptyset, 1}^{\neq 0} & =\left\{\left[a_{1}, a_{2}, b, c\right] \in \bar{M}_{\emptyset, 1}:\left[a_{1}, a_{2}, b, c\right] \in M_{\emptyset, 1} \text { or }\left(a_{1}, a_{2}\right) \neq(0,0)\right\}, \\
\tilde{D} & =\left\{(\tilde{m}, m) \in \bar{M}_{\{0\}, 1} \times \bar{M}_{\emptyset, 1}^{\neq 0}: \tilde{f}(\tilde{m}) \neq f(m)\right\} .
\end{aligned}
$$

Let $\tilde{\boxplus}: \tilde{D} \rightarrow \bar{M}_{\{0\}, 2}$ be defined as follows (where $\chi_{1}$ is as in Notation 7.4):

$$
\begin{aligned}
& {\left[\tilde{a}_{1}, \tilde{a}_{2}, \tilde{d}, \tilde{b}, \tilde{c}\right] \tilde{\boxplus}\left[a_{1}, a_{2}, b, c\right]} \\
& \quad=\left[\left(\begin{array}{cc}
\tilde{a}_{1} & 0 \\
0 & \chi_{1}^{-1} a_{1}
\end{array}\right),\left(\begin{array}{cc}
\tilde{a}_{2} & \frac{\tilde{b} c}{a_{1}-\tilde{a} \tilde{a}_{1}} \\
\frac{\chi_{1}^{-1} b \tilde{c}}{\tilde{d} \tilde{a}_{1}-a_{1}} & \chi_{1}^{-1} a_{2}
\end{array}\right),\left(\begin{array}{cc}
\tilde{d} & 0 \\
0 & \chi_{1}
\end{array}\right),\left(\begin{array}{c}
\tilde{b} \\
\chi_{1}^{-1} b
\end{array}\right),\left(\begin{array}{ll}
\tilde{c} & c
\end{array}\right)\right] .
\end{aligned}
$$

Proposition 7.30. The maps $\boxplus$ and $\tilde{\boxplus}$ are well defined and we have:

(1) The map $\boxplus$ is commutative.

(2) Let $\widetilde{\mathbb{C}}^{2}$ be the blowup of $\mathbb{C}^{2}$ at the origin. If we write $\bar{M}_{\emptyset, 1}=M_{\emptyset, 1} \cup \mathbb{C}^{2}$, $\bar{M}_{\emptyset, 1}^{\neq 0}=M_{\emptyset, 1} \cup\left(\mathbb{C}^{2} \backslash\{0\}\right)$ and $\bar{M}_{\{0\}, 1}=M_{\{0\}, 1} \cup \widetilde{\mathbb{C}}^{2}$ (see the stratification in equation (7.13)) then the restriction of $\boxplus$ and $\tilde{\boxplus}$ to the strata indicated below is given by the canonical maps:

$$
\begin{aligned}
& \boxplus:\left(M_{\emptyset, 1} \times \mathbb{C}^{2}\right) \cap D \rightarrow M_{\emptyset, 1} \times \mathbb{C}^{2} \subset \bar{M}_{\emptyset, 2} \\
& \boxplus:\left(\mathbb{C}^{2} \times \mathbb{C}^{2}\right) \cap D \rightarrow S^{2} \mathbb{C}^{2} \subset \bar{M}_{\emptyset, 2} \\
& \tilde{\boxplus}:\left(M_{\{0\}, 1} \times \mathbb{C}^{2} \backslash\{0\}\right) \cap \tilde{D} \rightarrow M_{\{0\}, 1} \times \widetilde{\mathbb{C}}^{2} \subset \bar{M}_{\{0\}, 2} \\
& \tilde{\boxplus}:\left(\widetilde{\mathbb{C}}^{2} \times M_{\emptyset, 1}\right) \cap \tilde{D} \stackrel{\operatorname{id} \times \pi^{*}}{\longrightarrow} \widetilde{\mathbb{C}}^{2} \times M_{\{0\}, 1} \subset \bar{M}_{\{0\}, 2} \\
& \tilde{\boxplus}:\left(\widetilde{\mathbb{C}}^{2} \times \mathbb{C}^{2} \backslash\{0\}\right) \cap \tilde{D} \rightarrow \widetilde{\mathbb{C}}^{2} \times \widetilde{\mathbb{C}}^{2} \rightarrow S^{2} \widetilde{\mathbb{C}}^{2} \subset \bar{M}_{\{0\}, 2}
\end{aligned}
$$

(3) Given $\left(m, m^{\prime}\right) \in D$ we have $\tau_{z}^{*}\left(m \boxplus m^{\prime}\right)=\left(\tau_{z}^{*} m\right) \boxplus\left(\tau_{z}^{*} m^{\prime}\right)$. 
(4) Given $\left(m, m^{\prime}\right) \in D$ we have $\pi^{*}\left(m \boxplus m^{\prime}\right)=\left(\pi^{*} m\right) \tilde{\boxplus} m^{\prime}$.

(5) Given $(\tilde{m}, m) \in \tilde{D}$ we have $\pi_{\bullet}(\tilde{m} \tilde{\boxplus} m)=\left(\pi_{\bullet} \tilde{m}\right) \boxplus m$.

Proof. First we check that $\tilde{\boxplus}$ is well defined. Let $\tilde{r} \in \tilde{\mathcal{R}}_{1}$ and $r \in \mathcal{R}_{1}$ be such that $([\tilde{r}],[r]) \in \tilde{D}$, let $g, g_{1} \in G L\left(W_{1}\right)$ and let $g_{0} \in G L\left(W_{0}\right)$. Then, by equations (7.9) and (7.29) we get

$$
\left(\left(g_{0}, g_{1}\right) \cdot \tilde{r}\right) \tilde{\boxplus}(g \cdot r)=\left(\left(\begin{array}{cc}
g_{0} & 0 \\
0 & \chi_{1}^{-1} g \chi_{1}
\end{array}\right),\left(\begin{array}{cc}
g_{1} & 0 \\
0 & g
\end{array}\right)\right) \cdot(\tilde{r} \tilde{\boxplus} r)
$$

which shows $\tilde{\boxplus}$ is well defined in the quotient. We also need to check that $[\tilde{r}] \tilde{\boxplus}[r]$ satisfies conditions (7.2) and (7.3). The integrability condition is straightforward. Condition (7.2) is trivially satisfied if $\tilde{a}_{1} a_{1} \neq 0$ so assume $\tilde{a}_{1} a_{1}=0$. Since $\tilde{f}(\tilde{m}) \neq$ $f(m)$ we cannot have $\tilde{a}_{1}=a_{1}=0$. If $\tilde{a}_{1}=0$ then $a_{1} \neq 0$ and since $[\tilde{r}]$ satisfies condition (7.2), either $\tilde{a}_{2} \neq 0$ or $\tilde{b} \neq 0$. In either case $[\tilde{r}] \tilde{\boxplus}[r]$ satisfies condition (7.2). If $a_{1}=0$ then $\tilde{a}_{1} \neq 0$ and since $[r] \in \bar{M}_{\emptyset, 1}^{\neq 0}$, either $a_{2} \neq 0$ or $b \neq 0$. In either case condition (7.2) is satisfied. This shows that $\tilde{\boxplus}$ is well defined. The proof that $\boxplus$ is well defined is completely analogous. Statements (3), (4) and (5) follow easily from Propositions 7.11(3)(4) and Proposition 7.22 if $m, m^{\prime} \in M_{\emptyset, 1}$ and $\tilde{m} \in M_{\{0\}, 1}$, and the general case follows by continuity since $M_{\emptyset, 1} \subset \bar{M}_{\emptyset, 1}$ and $M_{\{0\}, 1} \subset \bar{M}_{\{0\}, 1}$ are dense. We prove (1) and (2):

(1) Let $r, r^{\prime} \in \mathcal{R}_{1}$ be such that $[r],\left[r^{\prime}\right] \in D$ and let $g=\left(\begin{array}{ll}0 & 1 \\ 1 & 0\end{array}\right) \in G L\left(W_{1}\right)$. Then $g^{-1}\left(r \boxplus r^{\prime}\right) g=r^{\prime} \boxplus r$ so, in the quotient, $[r] \boxplus\left[r^{\prime}\right]=\left[r^{\prime}\right] \boxplus[r]$.

(2) We only prove the statement for $\boxplus$ since the proof for $\tilde{\boxplus}$ is entirely analogous. Suppose $r^{\prime}=\left(a_{1}^{\prime}, a_{2}^{\prime},, b^{\prime}, c^{\prime}\right) \notin \mathcal{R}_{1}^{\text {reg }}$. Then we must have either $b^{\prime}=0$ or $c^{\prime}=0$. Assume $c^{\prime}=0$ (the proof for the case $b^{\prime}=0$ is completely analogous). Let $r=\left(a_{1}, a_{2}, b, c\right)$ and for each $\varepsilon>0$ let $g=\left(\begin{array}{ll}1 & 0 \\ 0 & \varepsilon\end{array}\right) \in G L\left(W_{1}\right)$. Then

$$
g \cdot\left(r \tilde{\boxplus} r^{\prime}\right)=\left(\left(\begin{array}{cc}
a_{1} & 0 \\
0 & a_{1}^{\prime}
\end{array}\right),\left(\begin{array}{cc}
a_{2} & 0 \\
\varepsilon \frac{b^{\prime} c}{a_{1}-a_{1}^{\prime}} & a_{2}^{\prime}
\end{array}\right),\left(\begin{array}{c}
b \\
\varepsilon b^{\prime}
\end{array}\right),\left(\begin{array}{ll}
c & 0
\end{array}\right)\right) .
$$

Taking the limit when $\varepsilon \rightarrow 0$ we get $r \boxplus r^{\prime} \sim\left(a_{1}, a_{2}, b, c\right) \oplus\left(a_{1}^{\prime}, a_{2}^{\prime}, 0,0\right)$ which corresponds to $\left([r],\left(a_{1}^{\prime}, a_{2}^{\prime}\right)\right) \in \bar{M}_{\emptyset, 2}$ if $r \in \mathcal{R}_{1}^{\text {reg }}$ or $\left(\left(a_{1}, a_{2}\right),\left(a_{1}^{\prime}, a_{2}^{\prime}\right)\right) \in$ $S^{2} \mathbb{C}^{2}$ if $r \notin \mathcal{R}_{1}^{\text {reg }}$ (see section $[7.2$ ).

7.6. The spaces $\mathfrak{M}_{\boldsymbol{I}}^{\mathbb{V}, \boldsymbol{U}}$. In order to extend the maps $\boxplus$, $\tilde{\boxplus}$ in Definition 7.27 to the moduli spaces $\mathfrak{M}_{I, 1}$ we will replace these moduli spaces by homeomorphic subspaces. Let $\pi_{J, I}^{*}$ be as in Definition 3.4(4). Identify the spaces $\mathfrak{M}_{\emptyset}$ and $M_{\emptyset}$ through the isomorphism $\psi_{\emptyset}$ (see equation (7.10). The maps $f$ and $\tilde{f}$ in equation (7.26) extend to maps $\mathfrak{M}_{I, 1} \rightarrow \mathbb{C}$ in the following sense:

Proposition 7.32. There is, for each finite set $I \subset \mathbb{C}^{2}$, a unique continuous map $f_{I}: \mathfrak{M}_{I, 1} \rightarrow \mathbb{C}$ with the property that $f_{\emptyset}=f$ and $f_{J}=f_{I} \circ \pi_{J, I}^{*}$ for any $J \subset I$.

Proof. Uniqueness follows because $\pi_{\emptyset, I}^{*} \mathfrak{M}_{\emptyset, 1}$ is dense in $\mathfrak{M}_{I, 1}$. Let $z=\left(z_{1}, z_{2}\right) \in \mathbb{C}^{2}$ and let $\psi_{\{z\}}$ be as in equation (17.25). We define $f_{z}=\left(\tilde{f} \circ \psi_{\{z\}}^{-1}\right)+z_{1}$. Then $f_{z} \circ \pi_{\emptyset, z}^{*}=f_{\emptyset}$. The open sets $\left\{\pi_{z, I}^{*} \mathfrak{M}_{z, 1}\right\}_{z \in I}$ form an open cover of $\mathfrak{M}_{I, 1}$ and the collection of maps $\left\{f_{z}\right\}_{z \in I}$ is compatible in the intersections (see Lemma 6.2) so $\left\{f_{z}\right\}_{z \in I}$ defines the required map $f_{I}$. 
Definition 7.33. We say an open set $U \subset \mathbb{C}$ is a Jordan open set if $U$ is the bounded region inside a Jordan curve.

Let $U \subset \mathbb{C}$ be a Jordan open set and let $I \subset U \times \mathbb{C}$ be a finite set. Let $\mathbb{V}$ be a finite dimensional complex Hermitian vector space. Then we define:

$$
\mathfrak{M}_{I, 1}^{\mathbb{V}, U}=f_{I}^{-1}(U), \quad \mathfrak{M}_{I, k}^{\mathbb{V}, U}=\mathfrak{M}_{I, k}^{\mathbb{V}} \quad(\text { for } k \neq 1) .
$$

Except for Proposition 7.46, we omit the vector space $\mathbb{V}$ from the notation.

Proposition 7.35. Let $U, I$ be as in Definition 7.33. Then there is a homeomorphism $\mathfrak{M}_{I}^{U} \cong \mathfrak{M}_{I}$ which is homotopic to the inclusion map.

Proof. The statement is a tautology in degrees $k \neq 1$ so let $k=1$. Let $p: \mathbb{C}^{2} \rightarrow \mathbb{C}$ be projection onto the first factor. First we show that there is a neighbourhood $U^{\prime}$ of $p(I)$ in $U$ and a homotopy $H: U \times[0,1] \rightarrow \mathbb{C}$ between the inclusion $U \rightarrow \mathbb{C}$ and a homeomorphism $U \cong \mathbb{C}$ such that $H(z, t)=z$ for all $z \in U^{\prime}$. By the Schoenflies Theorem there is a homeomorphism $h: \mathbb{C} \rightarrow \mathbb{C}$ such that $h(U)=D$ is the open unit disk. We let $H=h^{-1} \circ H_{D} \circ(h \times$ id $)$ where $H_{D}: D \times[0,1] \rightarrow \mathbb{C}$ is a homotopy between the inclusion and a homeomorphism $D \cong \mathbb{C}$ which is the identity on a smaller disk $D^{\prime} \subset D$ containing $h(p(I))$. Then $H$ has the required properties, with $U^{\prime}=h^{-1}\left(D^{\prime}\right)$.

Identifying $M_{\emptyset, 1}$ with $\mathfrak{M}_{\emptyset, 1}$, we define the homotopy $H_{\emptyset}: \mathfrak{M}_{\emptyset, 1}^{U} \times[0,1] \rightarrow \mathfrak{M}_{\emptyset, 1}$ by $H_{\emptyset}\left(\left[a_{1}, a_{2}, b, c\right], t\right)=\left[H\left(a_{1}, t\right), a_{2}, b, c\right]$. Then:

$$
m \in \mathfrak{M}_{\emptyset, 1}^{U^{\prime}} \Rightarrow H_{\emptyset}(m, t)=m .
$$

For each $z \in I$ let $\pi_{z}^{*}: \mathfrak{M}_{\emptyset, 1} \rightarrow \mathfrak{M}_{z, 1}$ and $\pi_{z \bullet}: \mathfrak{M}_{z, 1} \rightarrow \overline{\mathfrak{M}}_{\emptyset, 1}$ be the maps induced by the projection. From Proposition $7.24(4)$, if $[\tilde{r}] \in S_{0} M_{\{0\}, 1}$ then $f_{\{0\}}([\tilde{r}])=0$ so $S_{0} M_{\{0\}, 1} \subset M_{\{0\}, 1}^{U^{\prime}}$. Let $S_{0} \mathfrak{M}_{z, 1} \subset \mathfrak{M}_{z, 1}$ be the image of $S_{0} M_{\{0\}, 1}$ under the isomorphism $\psi_{\{z\}}: M_{\{0\}, 1} \rightarrow \mathfrak{M}_{z, 1}$. Then $S_{0} \mathfrak{M}_{z, 1} \subset \mathfrak{M}_{z, 1}^{U^{\prime}}$ and from Proposition 7.24 (3) we have $\pi_{z}^{*} \mathfrak{M}_{\emptyset, 1}^{U} \cup S_{0} \mathfrak{M}_{z, 1}=\mathfrak{M}_{z, 1}^{U}$ so:

$$
\pi_{z}^{*} \mathfrak{M}_{\emptyset, 1}^{U} \cup \mathfrak{M}_{z, 1}^{U^{\prime}}=\mathfrak{M}_{z, 1}^{U} .
$$

Define $\tilde{H}_{z}: \mathfrak{M}_{z, 1}^{U} \times[0,1] \rightarrow \mathfrak{M}_{z, 1}$ by

$$
\tilde{H}_{z}(\tilde{m}, t)= \begin{cases}\pi_{z}^{*} \circ H_{\emptyset}\left(\pi_{z} \bullet \tilde{m}, t\right) & \tilde{m} \in \pi_{z}^{*} \mathfrak{M}_{\emptyset, 1}^{U} ; \\ \tilde{m} & \tilde{m} \in \mathfrak{M}_{z, 1}^{U^{\prime}}\end{cases}
$$

We need to check that $\tilde{H}_{z}$ is well defined and continuous. From equation (7.18) it follows that, for any $m \in \mathfrak{M}_{\emptyset, 1}^{U}$ we have:

$$
\tilde{H}_{z}\left(\pi_{z}^{*} m, t\right)=\pi_{z}^{*} H_{\emptyset}(m, t) .
$$

If $\tilde{m}=\pi_{z}^{*} m \in \pi_{z}^{*} \mathfrak{M}_{\emptyset, 1}^{U} \cap \mathfrak{M}_{z, 1}^{U^{\prime}}$ then $m \in \mathfrak{M}_{\emptyset, 1}^{U^{\prime}}$ so, by equations (17.36) and (7.37):

$$
\tilde{H}_{z}\left(\pi_{z}^{*} m, t\right)=\pi_{z}^{*} H_{\emptyset}(m, t)=\pi_{z}^{*} m
$$

and $\tilde{H}_{z}$ is well defined. By Lemma 6.2 the set $\pi_{z}^{*} \mathfrak{M}_{\emptyset, 1}^{U}$ is open, and $\mathfrak{M}_{\{0\}, 1}^{U^{\prime}}$ is clearly open so $\tilde{H}_{z}$ is continuous. From equation (7.37) it follows that the homotopies $\left\{H_{z}\right\}_{z \in I}$ patch together to define the homotopy $H_{I}: \mathfrak{M}_{I, 1}^{U} \times[0,1] \rightarrow \mathfrak{M}_{I, 1}$.

Proposition 7.38. Let $U_{1}, U_{2} \subset \mathbb{C}$ be disjoint open sets. Then: 
(1) The map $\boxplus: \bar{M}_{\emptyset, 1}^{U_{1}} \times \bar{M}_{\emptyset, 1}^{U_{2}} \rightarrow \bar{M}_{\emptyset, 2}$ is a homeomorphism onto the subspace $N_{0}=\left\{\left[a_{1}, a_{2}, b, c\right] \in \bar{M}_{\emptyset, 2}\right.$ : each $U_{i}$ contains exactly one eigenvalue of $\left.a_{1}\right\}$.

(2) If $0 \in U_{1}$ then the map $\tilde{\boxplus}: \bar{M}_{\{0\}, 1}^{U_{1}} \times \bar{M}_{\emptyset, 1}^{U_{2}} \rightarrow \bar{M}_{\{0\}, 2}$ is a homeomorphism onto the subspace

$N_{1}=\left\{\left(a_{1}, a_{2}, d, b, c\right) \in \bar{M}_{\{0\}, 2}\right.$ : each $U_{i}$ contains exactly one eigenvalue of $\left.d a_{1}\right\}$.

Proof. This is proven in [42, Proposition 4.5(1)] in the non-degenerate case and when $U_{1}$ and $U_{2}$ are disjoint balls but the exact same proof works in our case. We sketch how the inverse of the map $\tilde{\boxplus}$ is constructed. Let $\tilde{m}=\left[\tilde{a}_{1}, \tilde{a}_{2}, \tilde{d}, \tilde{b}, \tilde{c}\right] \in N_{1}$. Then the eigenvalues of $\tilde{d} \tilde{a}_{1}$ are distinct. Fix an eigenvector basis for $\tilde{d} \tilde{a}_{1}$. Then each eigenvector $v$ determines, up to rescaling, an eigenvector of $\tilde{a}_{1} \tilde{d}$ with the same eigenvalue: if $\tilde{a}_{1} v \neq 0$, then we can take $w=\tilde{a}_{1} v$; if $\tilde{a}_{1} v=0$ then we choose $w$ generating the kernel of $\tilde{a}_{1} \tilde{d}$. With this choice of basis, $\tilde{m}$ is of the form

$$
\left(\begin{array}{cc}
\tilde{a}_{1}^{\prime} & 0 \\
0 & \chi_{1}^{-1} a_{1}^{\prime}
\end{array}\right),\left(\begin{array}{cc}
\tilde{a}_{2}^{\prime} & \frac{\tilde{b}^{\prime} c^{\prime}}{a_{1}^{\prime}-\tilde{d}^{\prime} \tilde{a}_{1}^{\prime}} \\
\frac{\chi_{1}^{-1} b^{\prime} \tilde{c}^{\prime}}{\tilde{d}^{\prime} \tilde{a}_{1}^{\prime}-a_{1}^{\prime}} & \chi_{1}^{-1} a_{2}^{\prime}
\end{array}\right),\left(\begin{array}{cc}
\tilde{d}^{\prime} & 0 \\
0 & \chi_{1}
\end{array}\right),\left(\begin{array}{c}
\tilde{b}^{\prime} \\
\chi_{1}^{-1} b^{\prime}
\end{array}\right),\left(\begin{array}{ll}
\tilde{c}^{\prime} & c^{\prime}
\end{array}\right)
$$

(compare with equation (7.29)) and the action of $G L\left(W_{0}\right) \times G L\left(W_{1}\right)$ reduces to the action of pairs of the form $\left(\left(\begin{array}{cc}g_{0} & 0 \\ 0 & \chi_{1}^{-1} g \chi_{1}\end{array}\right),\left(\begin{array}{cc}g_{1} & 0 \\ 0 & g\end{array}\right)\right)$ (compare with equation (7.31) $)$. From the matrices in equation (7.39) we can recover $\left[\tilde{a}_{1}^{\prime}, \tilde{a}_{2}^{\prime}, \tilde{d}^{\prime}, \tilde{b}^{\prime}, \tilde{c}^{\prime}\right] \in \bar{M}_{\{0\}, 1}$ and $\left[a_{1}^{\prime}, a_{2}^{\prime}, b^{\prime}, c^{\prime}\right] \in \bar{M}_{\emptyset, 1}$.

7.7. The product maps. Given disjoint finite sets $I_{1}, I_{2}$ and disjoint open sets $U_{1}, U_{2}$ containing them we wish to define maps $\boxplus_{I_{1}, I_{2}}: \mathfrak{M}_{I_{1}, 1}^{U_{1}} \times \mathfrak{M}_{I_{2}, 1}^{U_{2}} \rightarrow \mathfrak{M}_{I_{1} \cup I_{2}, 2}$. Since the space $\mathfrak{M}_{I, 1}$ has an open cover $\left\{\pi_{x, I}^{*} \mathfrak{M}_{x, 1}\right\}_{x \in I}$, we only need to define the map in the cases when $I_{1}$ and $I_{2}$ have 1 or less elements and check the compatibility on the intersections. Let $\psi_{\{z\}}$ be as in equation (7.25). Identifying $M_{\emptyset}$ with $\mathfrak{M}_{\emptyset}$, we define:

$$
\boxplus_{\emptyset, \emptyset}=\boxplus, \quad \boxplus_{z, \emptyset}=\psi_{\{z\}} \circ \tilde{\boxplus} \circ\left(\psi_{\{z\}}^{-1} \times \tau_{z}^{*}\right), \quad \boxplus_{\emptyset, z}=\boxplus_{z, \emptyset} \circ \sigma
$$

where $\sigma$ is the permutation $\sigma\left(m_{\emptyset}, m_{z}\right)=\left(m_{z}, m_{\emptyset}\right)$. Then it follows from Proposition $7.30(3)(4)$ that $\left(\pi_{\emptyset, z}^{*} m\right) \boxplus_{z, \emptyset} m^{\prime}=\pi_{\emptyset, z}^{*}\left(m \boxplus_{\emptyset, \emptyset} m^{\prime}\right)$. Next we consider the case where $I_{1}$ and $I_{2}$ are both singletons:

Proposition 7.41. Let $U_{1}, U_{2} \subset \mathbb{C}$ be disjoint open sets, let $x \in U_{1} \times \mathbb{C}, y \in U_{2} \times \mathbb{C}$ and let $I=\{x, y\}$. There is an open embedding $\boxplus_{x, y}: \mathfrak{M}_{x, 1}^{U_{1}} \times \mathfrak{M}_{y, 1}^{U_{2}} \rightarrow \mathfrak{M}_{I, 2}$ such that the following diagram commutes:

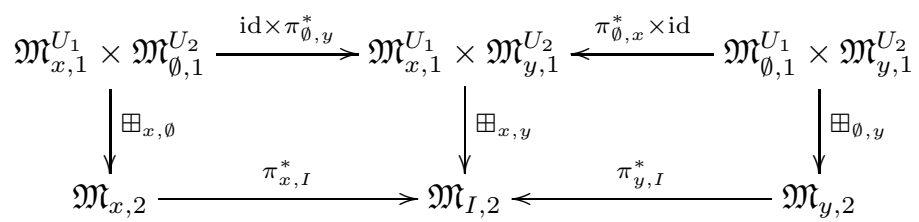

Furthermore, the image of $\boxplus_{x, y}$ is given by

$$
\operatorname{Im} \boxplus_{x, y}=\left(\pi_{x, I}^{*} N_{x} \cup \pi_{y, I}^{*} N_{y} \cup C\right) \cap \mathfrak{M}_{I, 2}
$$

where $N_{x}, N_{y}$ are the images of $\boxplus_{x, \emptyset}$ and $\boxplus_{\emptyset, y}$ respectively, and $C$ is the complement of $\pi_{x, I}^{*} \mathfrak{M}_{x, 2} \cup \pi_{y, I}^{*} \mathfrak{M}_{y, 2}$ in $\mathfrak{M}_{I, 2}$. 
Proof. The proof follows the same lines as the proof of Proposition 4.9 in [42. Given $\tilde{m}_{x} \in \mathfrak{M}_{x, 1}^{U_{1}}$ and $\tilde{m}_{y} \in \mathfrak{M}_{y, 1}^{U_{2}}$ we define $m=\tilde{m}_{x} \boxplus_{x, y} \tilde{m}_{y}$ as the unique solution of the system of equations:

$$
\begin{aligned}
& \left(\pi_{x, I}\right) \cdot m=\tilde{m}_{x} \boxplus_{x, \emptyset}\left(\pi_{\emptyset, y}\right) \cdot \tilde{m}_{y} \\
& \left(\pi_{y, I}\right) \bullet m=\left(\pi_{\emptyset, x}\right) \bullet \tilde{m}_{x} \boxplus_{\emptyset, y} \tilde{m}_{y}
\end{aligned}
$$

We need to prove existence and uniqueness of solution. We have two cases (see Proposition $7.24(3))$ :

(1) Suppose that $\tilde{m}_{x}=\pi_{\emptyset, x}^{*} m_{x}$ for some $m_{x} \in \mathfrak{M}_{\emptyset, 1}$. Then, by Proposition 7.16. equation (7.43) becomes $\left(\pi_{y, I}\right) \bullet m=m_{x} \boxplus_{\emptyset, y} \tilde{m}_{y} \in \mathfrak{M}_{y, 2}$ which, by Proposition 7.20 , is equivalent to:

$$
m=\pi_{y, I}^{*}\left(\pi_{y, I}\right) \cdot m=\pi_{y, I}^{*}\left(m_{x} \boxplus_{\emptyset, y} \tilde{m}_{y}\right) .
$$

This shows uniqueness of the solution. To show existence we must check that the solution of equation (7.43) given in equation (7.44) satisfies also equation (7.42). Applying Corollary 7.21 and Proposition 7.30(4)(5) we get

$$
\begin{aligned}
\left(\pi_{x, I}\right) \bullet\left(\pi_{y, I}\right)^{*}\left(m_{x} \boxplus_{\emptyset, y} \tilde{m}_{y}\right)=\left(\pi_{\emptyset, x}\right)^{*}\left(\pi_{\emptyset, y}\right) \bullet\left(m_{x} \boxplus_{\emptyset, y} \tilde{m}_{y}\right) & \\
& =\left(\pi_{\emptyset, x}\right)^{*}\left(m_{x} \boxplus_{\emptyset, \emptyset}\left(\pi_{\emptyset, y}\right) \bullet \tilde{m}_{y}\right)=\left(\pi_{\emptyset, x}^{*} m_{x}\right) \boxplus_{x, \emptyset}\left(\pi_{\emptyset, y}\right) \bullet \tilde{m}_{y} .
\end{aligned}
$$

The case where $\tilde{m}_{y}=\pi_{\emptyset, y}^{*} m_{y}$ for some $m_{y} \in \mathfrak{M}_{\emptyset, 1}$ is completely analogous.

(2) Suppose $\tilde{m}_{x} \in S_{0} \mathfrak{M}_{x, 1}$ and $\tilde{m}_{y} \in S_{0} \mathfrak{M}_{y, 1}$ (see Definition 7.23). Then, by Proposition 7.24 (2) we have $\pi_{\emptyset, x} \bullet \tilde{m}_{x}=x$ and $\pi_{\emptyset, y} \bullet \tilde{m}_{y}=y$ so by Proposition $7.30(2)$

$$
\begin{aligned}
& \left(\pi_{x, I}\right) \bullet m=\tilde{m}_{x} \boxplus_{x, \emptyset}\left(\pi_{\emptyset, y} \bullet \tilde{m}_{y}\right)=\left(\tilde{m}_{x}, y\right) \in \mathfrak{M}_{x, 1} \times \mathbb{C}^{2} \subset \overline{\mathfrak{M}}_{x, 2} \\
& \left(\pi_{y, I}\right) \bullet m=\left(\pi_{\emptyset, x} \bullet \tilde{m}_{x}\right) \boxplus_{\emptyset, y} \tilde{m}_{y}=\left(\tilde{m}_{y}, x\right) \in \mathfrak{M}_{y, 1} \times \mathbb{C}^{2} \subset \overline{\mathfrak{M}}_{y, 2}
\end{aligned}
$$

and these equations are equivalent to

$$
\left(\pi_{x, I}\right)_{*}^{\vee \vee} m=\tilde{m}_{x}, \quad\left(\pi_{y, I}\right)_{*}^{\vee \vee} m=\tilde{m}_{y} .
$$

But the map $\left(\pi_{x, I}\right)_{*}^{\vee \vee} \times\left(\pi_{y, I}\right)_{*}^{\vee \vee}$ is a homeomorphism by [42, Proposition 4.3], so this system of equations has a unique solution, namely:

$$
m=\left(\left(\pi_{x, I}\right)_{*}^{\vee \vee} \times\left(\pi_{y, I}\right)_{*}^{\vee \vee}\right)^{-1}\left(\tilde{m}_{x}, \tilde{m}_{y}\right) .
$$

The statement about the image of $\boxplus_{x, y}$ easily follows from (1) and (2) above. Now we must show continuity. Consider the Gieseker compactification $\overline{\mathfrak{M}}_{I, 2}$ Gie , defined as the closure of $\mathfrak{M}_{I, 2}$ inside the moduli space of framed sheaves [41, section 2.3], 25. Theorem 1.21]. Pick sequences $\tilde{m}_{k} \in \mathfrak{M}_{x, 1}, \tilde{m}_{k}^{\prime} \in \mathfrak{M}_{y, 1}$ converging respectively to $\tilde{m}, \tilde{m}^{\prime}$. If $\tilde{m}_{k} \boxplus_{x, y} \tilde{m}_{k}^{\prime}$ doesn't converge to $\tilde{m} \boxplus_{x, y} \tilde{m}^{\prime}$ then, by compactness, there is a subsequence $\tilde{m}_{k_{n}} \boxplus_{x, y} \tilde{m}_{k_{n}}^{\prime}$ converging to a framed sheaf $(\mathcal{E}, \phi)$ over $\tilde{\mathbb{P}}_{I}^{2}$, with $(\mathcal{E}, \phi) \neq \tilde{m} \boxplus_{x, y} \tilde{m}^{\prime}$. By the continuity of the direct image maps (see [41, appendix $\mathrm{D}]$ ) and pullback maps, equations (7.42) and (7.43) imply that

$$
\left(\pi_{x, I}\right) \bullet \mathcal{E}=\tilde{m} \boxplus_{x, \emptyset}\left(\pi_{\emptyset, y}\right) \bullet \tilde{m}^{\prime}, \quad\left(\pi_{y, I}\right) \bullet \mathcal{E}=\left(\pi_{\emptyset, x}\right) \bullet \tilde{m} \boxplus_{\emptyset, y} \tilde{m}^{\prime} .
$$

The first equation implies that the sheaf $\mathcal{E}$ is locally free away from $y$ and from the second equation it follows that $\mathcal{E}$ is locally free away from $x$, so $\mathcal{E}$ is locally free, and hence a holomorphic vector bundle. Uniqueness of solution implies that $\mathcal{E}=\tilde{m} \boxplus_{x, y} \tilde{m}^{\prime}$ which finishes the proof of continuity. 
It remains to be shown that the inverse is also continuous. Consider sequences $\tilde{m}_{k} \in \mathfrak{M}_{x, 1}$ and $\tilde{m}_{k}^{\prime} \in \mathfrak{M}_{y, 1}$ such that $\tilde{m}_{k} \boxplus_{x, y} \tilde{m}_{k}^{\prime} \rightarrow \tilde{m} \boxplus_{x, y} \tilde{m}^{\prime}$. Then equations (7.42) and (7.43) imply that

$$
\begin{aligned}
& \lim _{k \rightarrow+\infty}\left(\tilde{m}_{k} \boxplus_{x, \emptyset}\left(\pi_{\emptyset, y}\right) \bullet \tilde{m}_{k}^{\prime}\right)=\tilde{m} \boxplus_{x, \emptyset}\left(\pi_{\emptyset, y}\right) \cdot \tilde{m}^{\prime} \\
& \lim _{k \rightarrow+\infty}\left(\left(\pi_{\emptyset, x}\right) \bullet \tilde{m}_{k} \boxplus_{\emptyset, y} \tilde{m}_{k}^{\prime}\right)=\left(\pi_{\emptyset, x}\right) \bullet \tilde{m} \boxplus_{\emptyset, y} \tilde{m}^{\prime}
\end{aligned}
$$

By Proposition 7.38 the maps $\boxplus_{x, \emptyset}$ and $\boxplus_{\emptyset, y}$ are embeddings so $\tilde{m}_{k} \rightarrow \tilde{m}$ and $\tilde{m}_{k}^{\prime} \rightarrow \tilde{m}^{\prime}$, which finishes the proof.

Corollary 7.45. The collection $\left\{\pi_{x, I}^{*} \mathfrak{M}_{x, 2}, \pi_{y, I}^{*} \mathfrak{M}_{y, 2}, \operatorname{Im} \boxplus_{x, y}\right\}$ is an open cover of $\mathfrak{M}_{I, 2}$.

Proof. This is [42, Theorem 4.1] if we take $U_{1}$ and $U_{2}$ to be open disks around $x$ and $y$ respectively (see [42, Definition 4.1]): with the notation in [42] we have $A_{L}=\pi_{x, I}^{*} \mathfrak{M}_{x, 2}, A_{R}=\pi_{y, I}^{*} \mathfrak{M}_{y, 2}$ and $N_{2}=\operatorname{Im} \boxplus_{x, y}$. For general $U_{1}$ and $U_{2}$ the only thing that needs to be checked is that $\operatorname{Im} \boxplus_{x, y}$ is open. This is [42, Proposition 4.8], whose proof only uses the fact that $x \in U_{1} \times \mathbb{C}$ and $y \in U_{2} \times \mathbb{C}$.

Proposition 7.46. Let $k_{1}, \ldots, k_{n}$ be nonnegative integers such that $k=\sum k_{i} \leq 2$ and let $U_{1}, \ldots, U_{n} \subset \mathbb{C}$ be pairwise disjoint open sets all contained in an open set $U \subset \mathbb{C}$. For each $n$-tuple of pairwise disjoint finite sets $\mathbf{I}=\left(I_{1}, \ldots, I_{n}\right)$ such that $I_{i} \subset U_{i} \times \mathbb{C}$ there is a map $\boxplus_{\mathbf{I}}: \mathfrak{M}_{I_{1}, k_{1}}^{\mathbb{V}, U_{1}} \times \cdots \times \mathfrak{M}_{I_{n}, k_{n}}^{\mathbb{V}, U_{n}} \rightarrow \mathfrak{M}_{I, k}^{\mathbb{V}, U}$ (with $I=\bigcup_{i} I_{i}$ ) such that:

(1) Given any $\mathbf{J}=\left(J_{1}, \ldots, J_{n}\right)$ with $J_{i} \subset I_{i}$ for any $i$, we have

$$
\left.\pi_{J, I}^{*} \circ \boxplus_{\mathbf{J}}=\boxplus_{\mathbf{I}} \circ\left(\pi_{J_{1}, I_{1}}^{*} \times \cdots \times \pi_{J_{n}, I_{n}}^{*}\right) \quad \text { (where } J=\bigcup_{i} J_{i}\right) .
$$

(2) Given a linear isometry $\alpha: \mathbb{V} \rightarrow \mathbb{V}^{\prime}$, the following diagram is commutative:

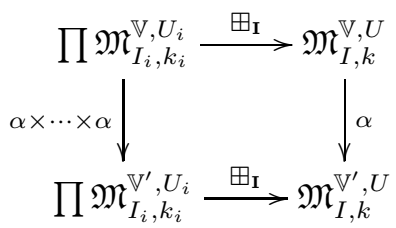

(3) Let $\imath_{i}: \mathbb{V} \rightarrow \mathbb{V}^{m}$ be the inclusion onto the $i$-th factor and let $\omega$ be the map induced by pullback and Whitney sum. Then we have a commutative diagram

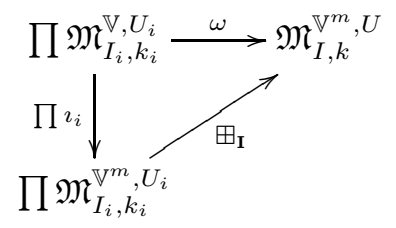


(4) Given a permutation $\sigma \in \Sigma_{n}$, let $\sigma \mathbf{I}=\left(I_{\sigma(1)}, \ldots, I_{\sigma(n)}\right)$. Then the following diagram is commutative:

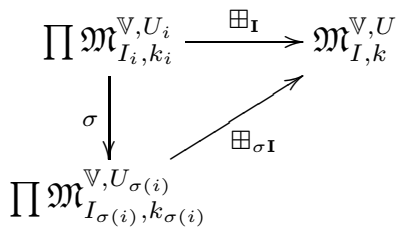

(5) The maps $\boxplus_{\mathbf{I}}$ are associative, that is, if $m<n$ then

$$
\boxplus_{\mathbf{I}}=\boxplus_{I_{1} \cup \ldots \cup I_{m}, I_{m+1}, \ldots, I_{n}} \circ\left(\boxplus_{I_{1}, \ldots, I_{m}} \times \mathrm{id}\right)
$$

Proof. If $k_{i}=k$ and $k_{j}=0$ for $j \neq i$ then $\boxplus_{\mathbf{I}}$ is defined by pullback:

$$
\boxplus_{\mathbf{I}}=\pi_{I_{i}, I}^{*}: \mathfrak{M}_{I_{i}, k}^{\mathbb{V}, U_{i}} \rightarrow \mathfrak{M}_{I, k}^{U}
$$

The only other case is the one where $k_{i}=k_{j}=1$ for some $i \neq j$ and $k=2$. The map $\boxplus_{\mathbf{I}}$ will be defined as the composition of a certain map $\boxplus_{I_{i}, I_{j}}: \mathfrak{M}_{I_{i}, 1}^{\mathbb{V}, U_{i}} \times \mathfrak{M}_{I_{j}, 1}^{\mathbb{V}, U_{j}} \rightarrow$ $\mathfrak{M}_{I_{i} \cup I_{j}, 2}^{\mathbb{V}, U}$ with pullback $\pi_{I_{i} \cup I_{j}, I}^{*}: \mathfrak{M}_{I_{i} \cup I_{j}, 2}^{\mathbb{V}, U} \rightarrow \mathfrak{M}_{I, 2}^{\mathbb{V}, U}$. It remains to define the maps $\boxplus_{I_{i}, I_{j}}$. Using the open cover from Lemma 6.2 we can reduce to the case where $I_{i}$ and $I_{j}$ are either empty or have only one element. These cases were taken care of in equation (7.40) and Proposition 7.41. Property (1) is clear and property (5) follows easily from (1). It remains to prove properties (2), (3) and (4). First we consider the case when $I=\emptyset$.

(2) Follows immediately from Proposition 7.11 (1) and the definition of $\boxplus$ (equation (7.28)).

(3) Let $m=\left[a_{1}, a_{2}, b, c\right] \in M_{\emptyset, 1}^{\mathbb{V}, U_{i}}$ and $m^{\prime}=\left[a_{1}^{\prime}, a_{2}^{\prime}, b^{\prime}, c^{\prime}\right] \in M_{\emptyset, 1}^{\mathbb{V}, U_{j}}$. By Proposition [7.11(1) we have $\imath_{i}(m)=\left[a_{1}, a_{2}, b \imath_{i}^{*}, \imath_{i} c\right]$ and $\imath_{j}\left(m^{\prime}\right)=\left[a_{1}^{\prime}, a_{2}^{\prime}, b^{\prime} \imath_{j}^{*}, \imath_{j} c^{\prime}\right]$. But now $b \imath_{i}^{*} \imath_{j} c^{\prime}=0$ and $b^{\prime} \imath_{j}^{*} \imath_{i} c=0$ from which it follows that the matrices in equation (7.28) are block diagonal and hence $\imath_{i}(m) \boxplus \imath_{j}\left(m^{\prime}\right)=$ $\imath_{i}(m) \oplus \imath_{j}\left(m^{\prime}\right)$ which, by Proposition 7.11 (2), corresponds to Whitney sum.

(4) This was proven in Proposition 7.30(1).

The results extend to the general case by continuity, since $\pi_{\emptyset, I_{i}}^{*} \mathfrak{M}_{\emptyset, 1}^{\mathbb{V}, U_{i}}$ is dense in $\mathfrak{M}_{I_{i}, 1}^{\mathbb{V}, U_{i}}$.

\section{The BAR CONSTRUCTIONS IN FINITE RANK}

The objective of this section is to prove Theorem 1.1. Up until section 8.7 we work with a fixed finite dimensional complex Hermitian vector space $\mathbb{V}$ which we omit from the notation. First we define, for each finite set $L \subset \mathbb{C}^{2}$, the degree 1 and 2 components of the bar construction (see section 2.1.3):

$$
B_{L}(\bullet)=\mathrm{B} \bullet\left(\mathfrak{M}_{\emptyset}, \prod_{x \in L} \mathfrak{M}_{\emptyset}, \prod_{x \in L} \mathfrak{M}_{x}\right)
$$


and, given disjoint finite sets $J$ and $K$, the degree 1 and 2 components of the bar constructions and the maps appearing in the following diagram:

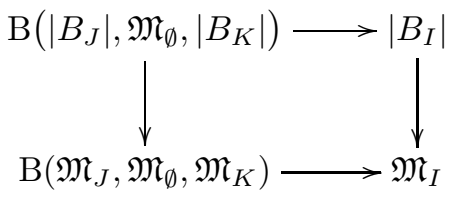

For each Jordan open set $U$ (see Definition 7.33) and each finite set $I \subset U \times \mathbb{C}$, let $\mathfrak{M}_{I}^{U}$ be as in equation (7.34). The face maps will be defined using the gluing maps $\boxplus_{\mathbf{I}}$ from Proposition 7.46. In degree $k=1$ all non-trivial gluing maps are pullback maps. In degree $k=2$ we will need:

- The pullback maps $\pi_{J, I}^{*}: \mathfrak{M}_{J}^{U_{1}} \rightarrow \mathfrak{M}_{I}^{U_{2}}$, with $J \subset I$ and $U_{1} \subset U_{2}$, obtained by restriction of the pullback maps in Definition 3.4(4);

- The gluing maps $\boxplus_{I_{1}, I_{2}}: \mathfrak{M}_{I_{1}, 1}^{U_{1}} \times \mathfrak{M}_{I_{2}, 1}^{U_{2}} \rightarrow \mathfrak{M}_{I_{1} \cup I_{2}, 2}^{U}$, with $U_{1}, U_{2} \subset U$ and $U_{1} \cap U_{2}=\emptyset$

- The inclusion maps $i: \mathfrak{M}_{I}^{U_{1}} \rightarrow \mathfrak{M}_{I}^{U_{2}}$, with $U_{1} \subset U_{2}$.

The maps $\boxplus_{I_{1}, I_{2}}$ require a choice of pairs of disjoint Jordan open sets $U_{1}, U_{2}$, and for the inclusion maps and pullback maps to be well defined we also need larger open sets containing certain families of open sets. We will now make these observations precise. Fix disjoint finite sets $J, K \subset \mathbb{C}^{2}$ and let $I=J \cup K$. Let $p: \mathbb{C}^{2} \rightarrow \mathbb{C}$ be projection onto the first factor. Given $z \in \mathbb{C}$ let $\Re z, \Im z$ be respectively the real and imaginary parts of $z$. Since a biholomorphic map $f: \mathbb{C}^{2} \rightarrow \mathbb{C}^{2}$ induces isomorphisms $\mathfrak{M}_{I} \cong \mathfrak{M}_{f(I)}$, we may assume without loss of generality that:

The assignment $x \mapsto \Re p(x)$ (with $x \in I$ ) is one to one.

In degree $k=2$, our constructions depend on a choice of Jordan open sets:

$$
C, D_{x}, U_{L}, C_{L} \subset \mathbb{C} \quad \text { (for each } x \in I \text { and each } L \subset I, L \neq \emptyset \text { ) }
$$

satisfying the following properties, for all $L^{\prime}, L \subset I$ and all $x, y \in I$ with $x \neq y$ :

$$
\begin{aligned}
& C_{L} \subset U_{L} \cap C, \quad p(x) \in D_{x} \subset U_{x}, \quad L^{\prime} \subset L \Rightarrow U_{L^{\prime}} \subset U_{L} ; \\
& D_{x} \cap C=\emptyset, \quad U_{x} \cap U_{y}=\emptyset, \quad U_{J} \cap U_{K}=\emptyset .
\end{aligned}
$$

Remark 8.4. Theorem 1.1, proved below, shows that the homotopy type of the spaces $\left|B_{L}\right|$ is independent of the choice of open sets.

Proposition 8.5. Given disjoint finite sets $J$ and $K$ such that $I=J \cup K$ satisfies condition (8.2), there are Jordan open sets satisfying conditions (8.3).

Proof. Fix $\delta<\frac{1}{2} \min \{|\Re p(x)-\Re p(y)|: x, y \in I, x \neq y\}$ and fix $r$ so that $|p(w)|<$ $r-3 \delta$ for any $w \in I$. Consider the rectangle

$$
R=\{z \in \mathbb{C}:-\delta<\Re z<\delta,-r+2 \delta<\Im z<r+\delta\} .
$$


For each $U \subset \mathbb{C}$ let $U^{*}=\{\bar{z}: z \in U\}$ be reflexion on the real axis. Consider the following open sets, where $x \in J, y \in K$ and $w \in I$ :

$$
\begin{aligned}
C_{J} & =\{z \in \mathbb{C}:-r-\delta<\Re z<r+\delta, r-\delta<\Im z<r+\delta\}, \quad C_{K}=C_{J}^{*} \\
C & =\{z \in \mathbb{C}: r-\delta<\Re z<r+\delta,-r-\delta<\Im z<r+\delta\} \cup C_{J} \cup C_{K} \\
U_{x} & =R+\Re p(x), \quad U_{y}=R^{*}+\Re p(y) \\
D_{w} & =\{z \in \mathbb{C}:|z-p(w)|<\delta\} \\
U_{J} & =C_{J} \cup\left(\cup_{x \in J} U_{x}\right), \quad U_{K}=C_{K} \cup\left(\bigcup_{y \in K} U_{y}\right), \quad U_{I}=C \cup\left(\cup_{w \in I} U_{w}\right)
\end{aligned}
$$

We define $U_{L}$ for $\# L>1$ as follows: If $L \subset J$ we take $U_{L}=U_{J}$; if $L \subset K$ we take $U_{L}=U_{K}$; otherwise $U_{L}=U_{I}$. Finally, we take $C_{L}=U_{L} \cap C$.

8.1. Structures in degrees up to $\boldsymbol{k}$. Although we are only able to define gluing maps up to degree 2 , we can still form a bar construction in degrees up to 2 by formally truncating the moduli spaces as follows:

Definition 8.6. Given a graded space $A=\bigsqcup_{n \geq 0} A_{n}$ with $A_{0}=\{*\}$, let $A_{\leq k}=$ $\{*\} \amalg A_{1} \amalg \cdots \amalg A_{k} \amalg\{*\} \amalg \cdots$ be the graded space obtained by collapsing to a point each summand $A_{n}$ where $n>k$.

Give the cartesian product $A_{\leq k} \times A_{\leq k}$ the natural grading. A monoid structure on $A$ in degrees up to $k$ is a monoid structure on the truncated graded space $A_{\leq k}$. For $k=2$ this is equivalent to a map

$$
q: A_{1} \times A_{1} \rightarrow A_{2} .
$$

A left action of $A$ on a graded space $N=\{*\} \amalg N_{1} \amalg N_{2} \amalg \cdots$ in degrees up to $k$ is a left action of $A_{\leq k}$ on $N_{\leq k}$. For $k=1$ this is just a map $Q_{1}^{\ell}: A_{1} \rightarrow N_{1}$. For $k=2$ giving such an action is equivalent to giving maps

$$
Q_{11}^{\ell}: A_{1} \times N_{1} \rightarrow N_{2}, \quad Q_{1}^{\ell}: A_{1} \rightarrow N_{1} \quad \text { and } \quad Q_{2}^{\ell}: A_{2} \rightarrow N_{2}
$$

satisfying the relation

$$
Q_{2}^{\ell}\left(q\left(r_{1}, r_{2}\right)\right)=Q_{11}^{\ell}\left(r_{1}, Q_{1}^{\ell}\left(r_{2}\right)\right) .
$$

We similarly define a right action of $A$ on a graded space $M$ in degrees up to $k$ as a right action of $A_{\leq k}$ on $M_{\leq k}$. For $k=2$ this action is given by maps

$$
Q_{11}^{r}: M_{1} \times A_{1} \rightarrow M_{2}, \quad Q_{1}^{r}: A_{1} \rightarrow M_{1} \quad \text { and } \quad Q_{2}^{r}: A_{2} \rightarrow M_{2}
$$

satisfying $Q_{2}^{r} \circ q=Q_{11}^{r} \circ\left(Q_{1}^{r} \times\right.$ id $)$. We represent the bar construction induced by the actions of $A$ on $M$ and $N$ by:

$$
\mathrm{B}(M, A, N)_{\leq k}=\mathrm{B}\left(M_{\leq k}, A_{\leq k}, N_{\leq k}\right)_{\leq k} .
$$

A map from $\mathrm{B}(M, A, N)$ to a graded space $Y$ can be defined by a map $\mathrm{B}_{0}(M, A, N) \rightarrow$ $Y$ coequalizing the face maps $\mathrm{B}_{1}(M, A, N) \rightrightarrows \mathrm{B}_{0}(M, A, N)$. In degree one such a map is defined by maps $W_{M}: M_{1} \rightarrow Y_{1}$ and $W_{N}: N_{1} \rightarrow Y_{1}$ satisfying the relation

$$
W_{N} \circ Q_{1}^{\ell}=W_{M} \circ Q_{1}^{r} \text {. }
$$

In degree 2 a map $\mathrm{B}(M, A, N) \rightarrow Y$ is defined by maps

$$
W_{M}: M_{2} \rightarrow Y_{2}, \quad W_{N}: N_{2} \rightarrow Y_{2}, \quad W_{11}: M_{1} \times N_{1} \rightarrow Y_{2},
$$


satisfying the relations

$$
\begin{aligned}
W_{M} \circ Q_{11}^{r} & =W_{11} \circ\left(\mathrm{id} \times Q_{1}^{\ell}\right), \\
W_{N} \circ Q_{11}^{\ell} & =W_{11} \circ\left(Q_{1}^{r} \times \mathrm{id}\right), \\
W_{M} \circ Q_{2}^{r} & =W_{N} \circ Q_{2}^{\ell} .
\end{aligned}
$$

8.2. Bar constructions in degree 1. In degree $k=1$, a choice of Jordan open sets is not necessary. However, we still need to be careful in how we define the degree 1 bar constructions because they will be used in degree 2 to define the upper row in diagram (8.1).

Let $J, K \subset \mathbb{C}^{2}$ be disjoint finite sets and let $I=J \cup K$. Given $L \subset I$ and $x \in L$ pick open sets $D_{x}$ and $U_{L}$ such that $p(x) \in D_{x}$ and $D_{x} \subset U_{L}$ whenever $x \in L$ (compare with conditions (8.3): in degree $k=1$ we don't need to require that $D_{x} \cap D_{y}=\emptyset$ for $\left.x \neq y\right)$. Given a finite set $L \subset I$ the maps

$$
Q_{1}^{\ell}:\left(\prod_{x \in L} \mathfrak{M}_{\emptyset}^{D_{x}}\right)_{1} \stackrel{\prod \pi_{\emptyset, x}^{*}}{\longrightarrow}\left(\prod_{x \in L} \mathfrak{M}_{x}^{D_{x}}\right)_{1} \quad \text { and } \quad Q_{1}^{r}:\left(\prod_{x \in L} \mathfrak{M}_{\emptyset}^{D_{x}}\right)_{1}=\coprod_{x \in L} \mathfrak{M}_{\emptyset, 1}^{D_{x}} \stackrel{i}{\rightarrow} \mathfrak{M}_{\emptyset, 1}^{U_{L}}
$$

(where $\left(\prod \mathfrak{M}\right)_{1}$ denotes the degree 1 component) define, in degree 1 , a left and a right action of $\prod \mathfrak{M}_{\emptyset}^{D_{x}}$ on $\prod \mathfrak{M}_{x}^{D_{x}}$ and $\mathfrak{M}_{\emptyset}^{U_{L}}$ respectively, giving rise to a bar construction in degree 1 which we represent by

$$
B_{L, 1}(\bullet)=\mathrm{B}_{\bullet}\left(\mathfrak{M}_{\emptyset}^{U_{L}}, \prod_{x \in L} \mathfrak{M}_{\emptyset}^{D_{x}}, \prod_{x \in L} \mathfrak{M}_{x}^{D_{x}}\right)_{1} .
$$

Notation 8.11. In the proof of Proposition 8.42 it will be important to distinguish between the following two homeomorphic summands in $B_{L, 1}(2)$ :

$$
\left(\left(\prod_{x \in L} \mathfrak{M}_{\emptyset}^{D_{x}}\right)_{1} \times *\right) \amalg\left(* \times\left(\prod_{x \in L} \mathfrak{M}_{\emptyset}^{D_{x}}\right)_{1}\right)=\left(\prod_{x \in L} \mathfrak{M}_{\emptyset}^{D_{x}} \times \prod_{x \in L} \mathfrak{M}_{\emptyset}^{D_{x}}\right)_{1} \subset B_{L, 1}(2) .
$$

We will write:

$$
\begin{aligned}
\left(\prod_{x \in L} \mathfrak{M}_{\emptyset}^{D_{x}}\right)_{1}^{\ell} & =\coprod_{x \in L} \mathfrak{M}_{\emptyset, 1}^{D_{x}, \ell}=\left(\prod_{x \in L} \mathfrak{M}_{\emptyset}^{D_{x}}\right)_{1} \times * ; \\
\left(\prod_{x \in L} \mathfrak{M}_{\emptyset}^{D_{x}}\right)_{1}^{r} & =\coprod_{x \in L} \mathfrak{M}_{\emptyset, 1}^{D_{x}, r}=* \times\left(\prod_{x \in L} \mathfrak{M}_{\emptyset}^{D_{x}}\right)_{1} .
\end{aligned}
$$

The maps

$$
W_{M}: \mathfrak{M}_{\emptyset, 1}^{U_{L}} \stackrel{\pi_{\emptyset, L}^{*}}{\longrightarrow} \mathfrak{M}_{L, 1}^{U_{L}} \quad \text { and } \quad W_{N}:\left(\prod_{x \in L} \mathfrak{M}_{x}^{D_{x}}\right)_{1}=\coprod_{x \in L} \mathfrak{M}_{x, 1}^{D_{x}} \stackrel{\pi_{x, L}^{*}}{\longrightarrow} \mathfrak{M}_{L, 1}^{U_{L}}
$$

satisfy relation (8.8) so they define a map

$$
h_{\boxplus}:\left|B_{L, 1}\right| \rightarrow \mathfrak{M}_{L, 1}^{U_{L}} .
$$

Remark 8.13. Let $\hat{B}_{L, 1}$ be the simplicial space obtained by taking $D_{x}=U_{L}=\mathbb{C}$. Then for any other choice of open sets $D_{x}$ and $U_{L}$ satisfying $p(x) \in D_{x} \subset U_{L}$, the maps of monoids and modules determined by the inclusions $D_{x}, U_{L} \subset \mathbb{C}$ induce a weak equivalence $B_{L, 1} \rightarrow \hat{B}_{L, 1}$ (see Proposition 7.35).

Now let $L_{1}, L_{2} \subset I$ be disjoint finite sets and let $L=L_{1} \cup L_{2}$. Let $U_{1}, U_{2}, C_{L}$ be Jordan open sets such that

$$
\left.L_{i} \subset U_{i} \times \mathbb{C} \quad \text { (with } i=1,2\right), \quad C_{L} \subset U_{1} \cap U_{2} \quad \text { and } \quad U_{1} \cup U_{2} \subset U_{L} .
$$


The maps

$$
Q_{1}^{\ell}: \mathfrak{M}_{\emptyset, 1}^{C_{L}} \stackrel{\pi_{\emptyset, L_{2}}^{*}}{\longrightarrow} \mathfrak{M}_{L_{2}, 1}^{U_{2}} \quad \text { and } \quad Q_{1}^{r}: \mathfrak{M}_{\emptyset, 1}^{C_{L}} \stackrel{\pi_{\emptyset, L_{1}}^{*}}{\longrightarrow} \mathfrak{M}_{L_{1}, 1}^{U_{1}}
$$

define, in degree 1 , left and right actions of $\mathfrak{M}_{\emptyset}^{C_{L}}$ on $\mathfrak{M}_{L_{2}}^{U_{2}}$ and $\mathfrak{M}_{L_{1}}^{U_{1}}$ respectively, giving rise to a bar construction $\mathrm{B}\left(\mathfrak{M}_{L_{1}}^{U_{1}}, \mathfrak{M}_{\emptyset}^{C_{L}}, \mathfrak{M}_{L_{2}}^{U_{2}}\right)$. The maps

$$
W_{M}: \mathfrak{M}_{L_{1}, 1}^{U_{1}} \stackrel{\pi_{L_{1}, L}^{*}}{\longrightarrow} \mathfrak{M}_{L, 1}^{U_{L}} \quad \text { and } \quad W_{N}: \mathfrak{M}_{L_{2}, 1}^{U_{2}} \stackrel{\pi_{L_{2}, L}^{*}}{\longrightarrow} \mathfrak{M}_{L, 1}^{U_{L}}
$$

define a map:

$$
f: \mathrm{B}\left(\mathfrak{M}_{L_{1}}^{U_{1}}, \mathfrak{M}_{\emptyset}^{C_{L}}, \mathfrak{M}_{L_{2}}^{U_{2}}\right)_{1} \rightarrow \mathfrak{M}_{L, 1}^{U_{L}}
$$

Consider now the case when $L_{1}=L_{2}=\emptyset$.

Proposition 8.16. Let $C_{L}, U_{1}, U_{2}, U_{L}$ be Jordan open sets satisfying relations (8.14). Then the map

$$
f: \mathrm{B}\left(\mathfrak{M}_{\emptyset, 1}^{U_{1}}, \mathfrak{M}_{\emptyset, 1}^{C_{L}}, \mathfrak{M}_{\emptyset, 1}^{U_{2}}\right) \rightarrow \mathfrak{M}_{\emptyset, 1}^{U_{L}}
$$

from equation (8.15) is a homotopy equivalence.

Proof. The maps of monoids and modules determined by the inclusions $C_{L}, U_{1}, U_{2} \subset$ $U_{L}$ induce a weak equivalence

$$
\mathrm{B}\left(\mathfrak{M}_{\emptyset, 1}^{U_{1}}, \mathfrak{M}_{\emptyset, 1}^{C_{L}}, \mathfrak{M}_{\emptyset, 1}^{U_{2}}\right) \simeq \mathrm{B}\left(\mathfrak{M}_{\emptyset}^{U_{L}}, \mathfrak{M}_{\emptyset}^{U_{L}}, \mathfrak{M}_{\emptyset}^{U_{L}}\right)_{1}
$$

and $f$ is the composition

$$
\mathrm{B}\left(\mathfrak{M}_{\emptyset, 1}^{U_{1}}, \mathfrak{M}_{\emptyset, 1}^{C_{L}}, \mathfrak{M}_{\emptyset, 1}^{U_{2}}\right) \stackrel{\simeq}{\longrightarrow} \mathrm{B}\left(\mathfrak{M}_{\emptyset}^{U_{L}}, \mathfrak{M}_{\emptyset}^{U_{L}}, \mathfrak{M}_{\emptyset}^{U_{L}}\right)_{1} \rightarrow \mathfrak{M}_{\emptyset, 1}^{U_{L}}
$$

which is a homotopy equivalence (Proposition 2.4).

8.3. The spaces $\left|\boldsymbol{B}_{\boldsymbol{L}, \mathbf{2}}\right|$. Fix open sets satisfying conditions (8.3), and for each open set $U$ in the chosen collection, fix a homeomorphism $\psi_{U}: \mathfrak{M}_{L}^{U} \cong \mathfrak{M}_{L}$ homotopic to the inclusion $\mathfrak{M}_{L}^{U} \rightarrow \mathfrak{M}_{L}$ (see Proposition 7.35). Choose some order for the elements of $I$. For each finite set $L \subset I$ we define a monoid structure on $\prod_{x \in L} \mathfrak{M}_{\emptyset}$
in degrees up to 2 by:

$$
\begin{gathered}
q:\left(\prod_{x \in L} \mathfrak{M}_{\emptyset}\right)_{1} \times\left(\prod_{x \in L} \mathfrak{M}_{\emptyset}\right)_{1} \stackrel{\psi_{C_{x}}^{-1} \times \psi_{D_{x}}^{-1}}{\longrightarrow}\left(\prod_{x \in L} \mathfrak{M}_{\emptyset}^{C_{x}}\right)_{1} \times\left(\prod_{x \in L} \mathfrak{M}_{\emptyset}^{D_{x}}\right)_{1} \\
=\coprod_{x \in L}\left(\mathfrak{M}_{\emptyset, 1}^{C_{x}} \times \mathfrak{M}_{\emptyset, 1}^{D_{x}}\right) \amalg \underset{\substack{x, y \in L \\
x \neq y}}{\coprod_{\emptyset \neq, 1}}\left(\mathfrak{M}_{\emptyset, 1}^{C_{y}} \times \mathfrak{M}_{\emptyset, 1}^{D_{x}}\right) \\
\stackrel{\boxplus_{\emptyset, \emptyset} \amalg i}{\longrightarrow}\left(\coprod_{x \in L} \mathfrak{M}_{\emptyset, 2}^{U_{x}}\right) \amalg \underset{\substack{x, y \in L \\
y<x}}{\coprod_{\emptyset, 1}}\left(\mathfrak{M}^{U_{y}} \times \mathfrak{M}_{\emptyset, 1}^{U_{x}}\right)=\left(\prod_{x \in L} \mathfrak{M}_{\emptyset}^{U_{x}}\right)_{2} \stackrel{\psi_{U_{x}}}{\longrightarrow}\left(\prod_{x \in L} \mathfrak{M}_{\emptyset}\right)_{2} .
\end{gathered}
$$

We define a left action of $\prod \mathfrak{M}_{\emptyset}$ on $\prod \mathfrak{M}_{x}$ in degrees up to 2 by conjugating the following maps with the homeomorphisms $\mathfrak{M}_{\emptyset, k}^{U} \cong \mathfrak{M}_{\emptyset, k}$ and $\mathfrak{M}_{x, k}^{U} \cong \mathfrak{M}_{x, k}$ :

$$
\begin{aligned}
& Q_{11}^{\ell}:\left(\prod_{x \in L} \mathfrak{M}_{\emptyset}^{C_{x}}\right)_{1} \times\left(\prod_{x \in L} \mathfrak{M}_{x}^{D_{x}}\right)_{1} \stackrel{\boxplus_{\emptyset, x} \amalg i}{\longrightarrow}\left(\prod_{x \in L} \mathfrak{M}_{x}^{U_{x}}\right)_{2} ; \\
& Q_{1}^{\ell}:\left(\prod_{x \in L} \mathfrak{M}_{\emptyset}^{D_{x}}\right)_{1} \stackrel{\prod \pi_{\emptyset, x}^{*}}{\longrightarrow}\left(\prod_{x \in L} \mathfrak{M}_{x}^{D_{x}}\right)_{1} ; \\
& Q_{2}^{\ell}:\left(\prod_{x \in L} \mathfrak{M}_{\emptyset}^{U_{x}}\right)_{2} \stackrel{\prod \pi_{\emptyset, x}^{*}}{\longrightarrow}\left(\prod_{x \in L} \mathfrak{M}_{x}^{U_{x}}\right)_{2},
\end{aligned}
$$


where $Q_{11}^{\ell}$ is defined in an analogous way to the map $q$ is equation (8.17). Relation (8.7) follows from Proposition 7.46(1).

Notation 8.19. We'll abuse notation and denote by $Q_{1}^{\ell}, Q_{2}^{\ell}$ and $Q_{11}^{\ell}$ both the maps in equation (8.18) and the maps obtained by conjugating them with the homeomorphisms $\psi_{U}$.

Remark 8.20. The monoid structure on $\prod \mathfrak{M}_{\emptyset}$ and its left action on $\prod \mathfrak{M}_{x}$ are obtained by conjugating the maps (in degree 2 ):

$$
\begin{gathered}
\prod_{x \in L} \boxplus_{\emptyset, \emptyset}: \prod_{x \in L}\left(\mathfrak{M}_{\emptyset}^{C_{x}} \times \mathfrak{M}_{\emptyset}^{D_{x}}\right) \rightarrow \prod_{x \in L} \mathfrak{M}_{\emptyset}^{U_{x}}, \\
\prod_{x \in L} \boxplus_{\emptyset, x}: \prod_{x \in L}\left(\mathfrak{M}_{\emptyset}^{C_{x}} \times \mathfrak{M}_{x}^{D_{x}}\right) \rightarrow \prod_{x \in L} \mathfrak{M}_{x}^{U_{x}}
\end{gathered}
$$

with the homeomorphisms $\psi_{U}$.

We define a right action of $\left(\prod \mathfrak{M}_{\emptyset}\right)_{\leq 2}$ on $\mathfrak{M}_{\emptyset, \leq 2}$ using the maps

$$
\begin{aligned}
& Q_{11}^{r}: \mathfrak{M}_{\emptyset, 1}^{C} \times\left(\prod_{x \in L} \mathfrak{M}_{\emptyset}^{D_{x}}\right)_{1} \stackrel{\boxplus_{\emptyset, \ldots, \emptyset}}{\longrightarrow} \mathfrak{M}_{\emptyset, 2} ; \\
& Q_{1}^{r}:\left(\prod_{x \in L} \mathfrak{M}_{\emptyset}^{C_{x}}\right)_{1} \stackrel{\boxplus_{\emptyset, \ldots, \emptyset}}{\longrightarrow} \mathfrak{M}_{\emptyset, 1}^{C} ; \\
& Q_{2}^{r}:\left(\prod_{x \in L} \mathfrak{M}_{\emptyset}^{U_{x}}\right)_{2} \stackrel{\boxplus_{\emptyset, \ldots, \emptyset}}{\longrightarrow} \mathfrak{M}_{\emptyset, 2} .
\end{aligned}
$$

Note that, from Proposition $7.30(1)$, the map $Q_{2}^{r}$ doesn't depend on the order of the factors in the product $\prod_{x \in L} \mathfrak{M}_{\emptyset}^{U_{x}}$. Relation (8.7) follows from this observation and the naturality of the maps $\boxplus_{\emptyset, \emptyset}$ with respect to inclusions. From the monoid structure and the actions just defined we get a bar construction $\mathrm{B} \bullet\left(\mathfrak{M}_{\emptyset}, \prod \mathfrak{M}_{\emptyset}, \prod \mathfrak{M}_{x}\right)$ in degree 2.

Remark 8.22. We are not interested in the degree $k=1$ summand of this bar construction, which is not the same as the one in equation (8.10).

Write $L=\left\{x_{1}, \ldots, x_{j}\right\}$ and let $\boxplus_{\mathbf{L}}=\boxplus_{x_{1}, \ldots, x_{j}}$. The maps

$$
\begin{aligned}
& W_{M}: \mathfrak{M}_{\emptyset, 2} \stackrel{\pi_{\emptyset, L}^{*}}{\longrightarrow} \mathfrak{M}_{L, 2} \\
& W_{N}:\left(\prod_{x \in L} \mathfrak{M}_{x}\right)_{2} \cong\left(\prod_{x \in L} \mathfrak{M}_{x}^{U_{x}}\right)_{2} \stackrel{\boxplus_{\mathbf{L}}}{\longrightarrow} \mathfrak{M}_{L, 2} \\
& W_{11}: \mathfrak{M}_{\emptyset, 1} \times\left(\prod_{x \in L} \mathfrak{M}_{x}\right)_{1} \cong \coprod_{x \in L}\left(\mathfrak{M}_{\emptyset, 1}^{C} \times \mathfrak{M}_{x, 1}^{D_{x}}\right) \stackrel{\pi_{x, I}^{*} \circ \boxplus_{\emptyset, x}}{\longrightarrow} \mathfrak{M}_{L, 2}
\end{aligned}
$$

satisfy relations (8.9), so they define a map

$$
h_{\boxplus}: \mathrm{B}\left(\mathfrak{M}_{\emptyset}, \prod \mathfrak{M}_{\emptyset}, \prod \mathfrak{M}_{x}\right)_{2} \rightarrow \mathfrak{M}_{L, 2} .
$$

The bar construction $\mathrm{B} \cdot\left(\mathfrak{M}_{\emptyset}, \prod \mathfrak{M}_{\emptyset}, \prod \mathfrak{M}_{x}\right)_{2}$ is independent of the choice of homeomorphisms $\psi_{U}$. To make this independence explicit we will introduce a 
simplicial space $B_{L, 2}(\bullet)$ homeomorphic to $\mathrm{B} \bullet\left(\mathfrak{M}_{\emptyset}, \prod \mathfrak{M}_{\emptyset}, \prod \mathfrak{M}_{x}\right)_{2}$. For each $n$ let $B_{L, 2}(n)=\mathfrak{M}_{\emptyset, 2} \amalg\left(\prod_{x \in L} \mathfrak{M}_{x}^{U_{x}}\right)_{2} \amalg \mathfrak{M}_{\emptyset, 1}^{C} \times\left(\prod_{x \in L} \mathfrak{M}_{x}^{D_{x}}\right)_{1}$

$$
\begin{array}{r}
\amalg \coprod_{i=1}^{n}\left(\left(\prod_{x \in L} \mathfrak{M}_{\emptyset}^{U_{x}}\right)_{2} \amalg \mathfrak{M}_{\emptyset, 1}^{C} \times\left(\prod_{x \in L} \mathfrak{M}_{\emptyset}^{D_{x}}\right)_{1} \amalg\left(\prod_{x \in L} \mathfrak{M}_{\emptyset}^{C_{x}}\right)_{1} \times\left(\prod_{x \in L} \mathfrak{M}_{x}^{D_{x}}\right)_{1}\right) \\
\amalg \coprod_{\substack{i, j=1 \\
i<j}}^{n}\left(\prod_{x \in L} \mathfrak{M}_{\emptyset}^{C_{x}}\right)_{1} \times\left(\prod_{x \in L} \mathfrak{M}_{\emptyset}^{D_{x}}\right)_{1}
\end{array}
$$

The maps $\psi_{U}$ induce, for each $n$, a homeomorphism $B_{L, 2}(n) \cong \mathrm{B}_{n}\left(\mathfrak{M}_{\emptyset}, \prod \mathfrak{M}_{\emptyset}, \prod \mathfrak{M}_{x}\right)_{2}$. These homeomorphisms induce a simplicial space structure on $B_{L, 2}$. Note that on morphisms $B_{L, 2}$ is defined using only the inclusions $i$, pullbacks and the gluing maps $\boxplus_{\emptyset, \emptyset}$ and $\boxplus_{\emptyset, x}$.

In the proof of Proposition 8.42 we will need another simplicial space $B_{L, 2}^{\mathrm{big}}$ homotopically equivalent to $B_{L, 2}$, which we now define. For each $x \in L$ let

$$
B_{x}=C \cup\left(\bigcup_{\substack{y \in I \\ y \neq x}} U_{y}\right)
$$

We define, for each $n$, a space $B_{L, 2}^{\mathrm{big}}(n)$ homeomorphic to $B_{L, 2}(n)$ as follows:

$$
B_{L, 2}^{\mathrm{big}}(n)=
$$

$$
\begin{aligned}
& \mathfrak{M}_{\emptyset, 2} \amalg\left(\prod_{x \in L} \mathfrak{M}_{x}^{U_{x}}\right)_{2} \amalg\left(\coprod_{x \in L} \mathfrak{M}_{\emptyset, 1}^{B_{x}} \times \mathfrak{M}_{x, 1}^{D_{x}}\right) \\
& \amalg \coprod_{i=1}^{n}\left(\left(\prod_{x \in L} \mathfrak{M}_{\emptyset}^{U_{x}}\right)_{2} \amalg\left(\coprod_{x \in L} \mathfrak{M}_{\emptyset, 1}^{B_{x}} \times \mathfrak{M}_{\emptyset, 1}^{D_{x}}\right) \amalg\left(\coprod_{x \in L} \mathfrak{M}_{\emptyset, 1}^{C_{x}} \times \mathfrak{M}_{x, 1}^{D_{x}}\right) \amalg\left(\coprod_{\substack{x, y \in L \\
y \neq x}} \mathfrak{M}_{\emptyset, 1}^{U_{y}} \times \mathfrak{M}_{x, 1}^{D_{x}}\right)\right) \\
& \amalg \coprod_{\substack{i, j=1 \\
i \neq j}}^{n}\left(\left(\coprod_{x \in L} \mathfrak{M}_{\emptyset, 1}^{C_{x}} \times \mathfrak{M}_{\emptyset, 1}^{D_{x}}\right) \amalg\left(\coprod_{y \neq x} \mathfrak{M}_{\emptyset, 1}^{U_{y}} \times \mathfrak{M}_{\emptyset, 1}^{D_{x}}\right)\right)
\end{aligned}
$$

For each $n$ we have a homotopy equivalence $B_{L, 2}(n) \rightarrow B_{L, 2}^{\text {big }}(n)$, defined as the identity on the $\mathfrak{M}_{\emptyset, 2},\left(\prod \mathfrak{M}_{x}^{U_{x}}\right)_{2}$ and $\left(\prod \mathfrak{M}_{\emptyset}^{U_{x}}\right)_{2}$ summands (see equation (8.25)) and defined on the remaining summands by the inclusion maps

$$
\begin{aligned}
\mathfrak{M}_{\emptyset, 1}^{C} \times\left(\prod_{x \in L} \mathfrak{M}_{x}^{D_{x}}\right)_{1} & =\coprod_{x \in L}\left(\mathfrak{M}_{\emptyset, 1}^{C} \times \mathfrak{M}_{x, 1}^{D_{x}}\right) \rightarrow \coprod_{x \in L}\left(\mathfrak{M}_{\emptyset, 1}^{B_{x}} \times \mathfrak{M}_{x, 1}^{D_{x}}\right) \\
\mathfrak{M}_{\emptyset, 1}^{C} \times\left(\prod_{x \in L} \mathfrak{M}_{\emptyset}^{D_{x}}\right)_{1} & =\coprod_{x \in L}\left(\mathfrak{M}_{\emptyset, 1}^{C} \times \mathfrak{M}_{\emptyset, 1}^{D_{x}}\right) \rightarrow \coprod_{x \in L}\left(\mathfrak{M}_{\emptyset, 1}^{B_{x}} \times \mathfrak{M}_{\emptyset, 1}^{D_{x}}\right) \\
\left(\prod_{x \in L} \mathfrak{M}_{\emptyset}^{C_{x}}\right)_{1} \times\left(\prod_{x \in L} \mathfrak{M}_{x}^{D_{x}}\right)_{1} & =\left(\coprod_{x \in L} \mathfrak{M}_{\emptyset, 1}^{C_{x}} \times \mathfrak{M}_{x, 1}^{D_{x}}\right) \amalg\left(\coprod_{x \neq y} \mathfrak{M}_{\emptyset, 1}^{C_{y}} \times \mathfrak{M}_{x, 1}^{D_{x}}\right) \\
& \rightarrow\left(\coprod_{x \in L} \mathfrak{M}_{\emptyset, 1}^{C_{x}} \times \mathfrak{M}_{x, 1}^{D_{x}}\right) \amalg\left(\coprod_{x \neq y} \mathfrak{M}_{\emptyset, 1}^{U_{y}} \times \mathfrak{M}_{x, 1}^{D_{x}}\right) \\
\left(\prod_{x \in L} \mathfrak{M}_{\emptyset}^{C_{x}}\right)_{1} \times\left(\prod_{x \in L} \mathfrak{M}_{\emptyset}^{D_{x}}\right)_{1} & =\left(\coprod_{x \in L} \mathfrak{M}_{\emptyset, 1}^{C_{x}} \times \mathfrak{M}_{\emptyset, 1}^{D_{x}}\right) \amalg\left(\coprod_{x \neq y} \mathfrak{M}_{\emptyset, 1}^{C_{y}} \times \mathfrak{M}_{\emptyset, 1}^{D_{x}}\right) \\
& \rightarrow\left(\coprod_{x \in L} \mathfrak{M}_{\emptyset, 1}^{C_{x}} \times \mathfrak{M}_{\emptyset, 1}^{D_{x}}\right) \amalg\left(\coprod_{x \neq y} \mathfrak{M}_{\emptyset, 1}^{U_{y}} \times \mathfrak{M}_{\emptyset, 1}^{D_{x}}\right)
\end{aligned}
$$

The face and degeneracy maps on $B_{L, 2}$ are defined using inclusions, pullbacks and the gluing maps. All these maps can be naturally extended to define a simplicial 
structure on $B_{L, 2}^{\mathrm{big}}$ : if we write

$$
\begin{aligned}
& \left(\prod_{x \in L} \mathfrak{M}_{x}^{U_{x}}\right)_{2}=\left(\coprod_{x \in L} \mathfrak{M}_{x, 2}^{U_{x}}\right) \amalg\left(\underset{\substack{x, y \in L \\
y<x}}{\coprod_{y, 1}} \mathfrak{M}_{y, 1}^{U_{y}} \times \mathfrak{M}_{x, 1}^{U_{x}}\right) \\
& \left(\prod_{x \in L} \mathfrak{M}_{\emptyset}^{U_{x}}\right)_{2}=\left(\coprod_{x \in L} \mathfrak{M}_{\emptyset, 2}^{U_{x}}\right) \amalg\left(\coprod_{\substack{x, y \in L \\
y<x}} \mathfrak{M}_{\emptyset, 1}^{U_{y}} \times \mathfrak{M}_{\emptyset, 1}^{U_{x}}\right)
\end{aligned}
$$

all the face maps in $B_{L, 2}^{\text {big }}$ between non-degenerate summands are represented in the diagram below:

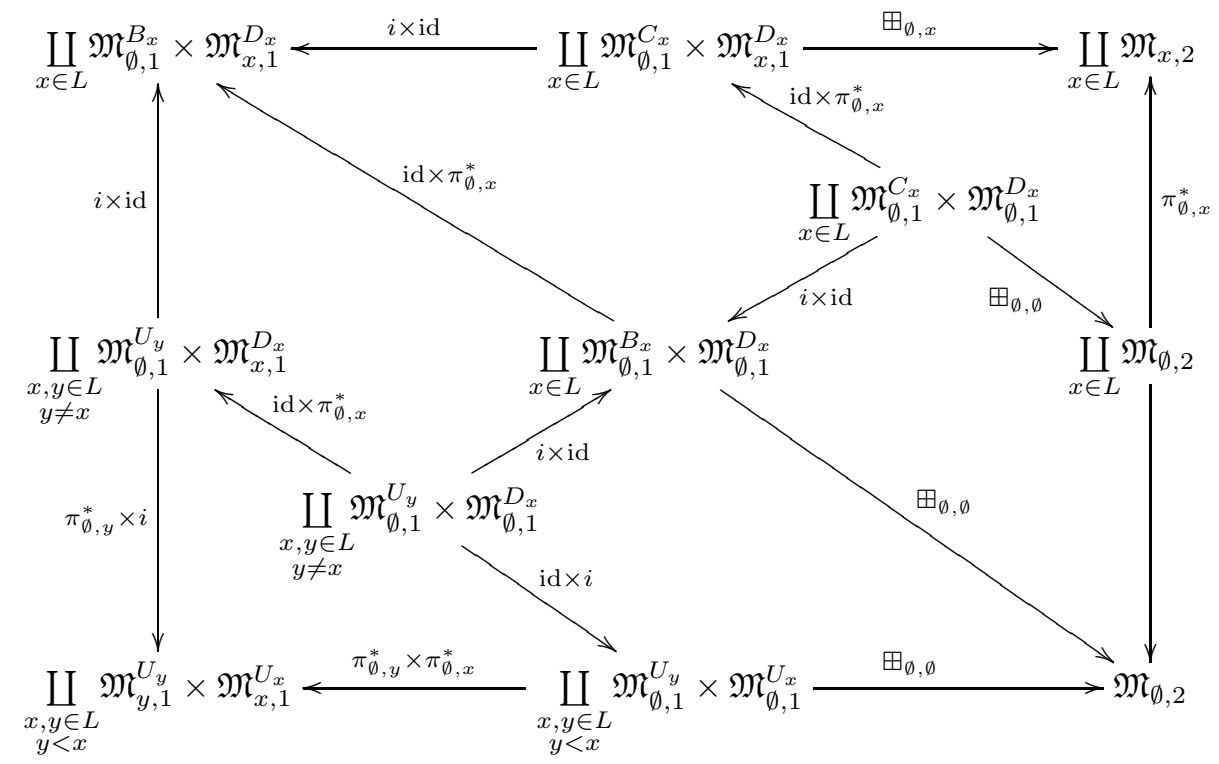

The inclusion maps in equation (8.27) induce a weak equivalence $B_{L, 2} \rightarrow B_{L, 2}^{\mathrm{big}}$.

The map $W_{11}$ in equation (8.23) extends to a map $\coprod \mathfrak{M}_{\emptyset, 1}^{B_{x}} \times \mathfrak{M}_{x, 1}^{D_{x}} \rightarrow \mathfrak{M}_{I, 2}$ so the map $h_{\boxplus}$ in equation (8.24) extends to a map

$$
h_{\boxplus}:\left|B_{L, 2}^{\mathrm{big}}\right| \rightarrow \mathfrak{M}_{L, 2} .
$$

8.4. The space $\mathbf{B}\left(\mathfrak{M}_{J}, \mathfrak{M}_{\emptyset}, \mathfrak{M}_{K}\right)$ in degree 2 . In this section and the next we fix finite sets $L_{1}, L_{2} \subset I$ such that $U_{L_{1}} \cap U_{L_{2}}=\emptyset$ (we are interested in 2 cases: the case where $\# L_{1}=\# L_{2}=1$ and the case where $L_{1}=J$ and $\left.L_{2}=K\right)$. We give $\mathfrak{M}_{\emptyset}$ the structure of a monoid in degrees up to 2 by the following map:

$$
q: \mathfrak{M}_{\emptyset, 1} \times \mathfrak{M}_{\emptyset, 1} \cong \mathfrak{M}_{\emptyset, 1}^{C_{L_{1}}} \times \mathfrak{M}_{\emptyset, 1}^{C_{L_{2}}} \stackrel{\boxplus_{\emptyset, \emptyset}}{\longrightarrow} \mathfrak{M}_{\emptyset, 2}
$$

We define a left action of $\mathfrak{M}_{\emptyset}$ on $\mathfrak{M}_{L_{2}}$ using the maps:

$$
\begin{aligned}
& Q_{11}^{\ell}: \mathfrak{M}_{\emptyset, 1} \times \mathfrak{M}_{L_{2}, 1} \cong \mathfrak{M}_{\emptyset, 1}^{C_{L_{1}}} \times \mathfrak{M}_{L_{2}, 1}^{U_{L_{2}}} \stackrel{\boxplus_{\emptyset, L_{2}}}{\longrightarrow} \mathfrak{M}_{L_{2}, 2}, \\
& Q_{1}^{\ell}: \mathfrak{M}_{\emptyset, 1} \cong \mathfrak{M}_{\emptyset, 1}^{C_{L_{2}}} \stackrel{\pi_{\emptyset, L_{2}}^{*}}{\longrightarrow} \mathfrak{M}_{L_{2}, 1}^{U_{L_{2}}} \cong \mathfrak{M}_{L_{2}, 1}, \\
& Q_{2}^{\ell}: \mathfrak{M}_{\emptyset, 2} \stackrel{\pi_{\emptyset, L_{2}}^{*}}{\longrightarrow} \mathfrak{M}_{L_{2}, 2},
\end{aligned}
$$


and we define in an analogous way a right action of $\mathfrak{M}_{\emptyset}$ on $\mathfrak{M}_{L_{1}}$ by

$$
\mathfrak{M}_{L_{1}, 1}^{U_{L_{1}}} \times \mathfrak{M}_{\emptyset, 1}^{C_{L_{2}}} \rightarrow \mathfrak{M}_{L_{1}, 2}, \quad \mathfrak{M}_{\emptyset, 1}^{C_{L_{1}}} \rightarrow \mathfrak{M}_{L_{1}, 1}^{U_{L_{1}}}, \quad \mathfrak{M}_{\emptyset, 2} \rightarrow \mathfrak{M}_{L_{1}, 2} .
$$

These actions give rise to a bar construction $\mathrm{B}\left(\mathfrak{M}_{L_{1}}, \mathfrak{M}_{\emptyset}, \mathfrak{M}_{L_{2}}\right)$. Let $L=L_{1} \cup L_{2}$. The maps

$$
\mathfrak{M}_{L_{1}, 2} \stackrel{\pi_{L_{1}, L}^{*}}{\longrightarrow} \mathfrak{M}_{L, 2}, \quad \mathfrak{M}_{L_{1}, 1}^{U_{L_{1}}} \times \mathfrak{M}_{L_{2}, 1}^{U_{L_{2}}} \stackrel{\boxplus_{L_{1}, L_{2}}}{\longrightarrow} \mathfrak{M}_{L, 2}, \quad \mathfrak{M}_{L_{2}, 2} \stackrel{\pi_{L_{2}, L}^{*}}{\longrightarrow} \mathfrak{M}_{L, 2}
$$

induce a map in degree 2 :

$$
f: \mathrm{B}\left(\mathfrak{M}_{L_{1}}, \mathfrak{M}_{\emptyset}, \mathfrak{M}_{L_{2}}\right) \rightarrow \mathfrak{M}_{L}
$$

Remark 8.34. The monoid structure and the actions defined in this section are well defined if we take $C_{L_{1}}=U_{L_{1}}$ and $C_{L_{2}}=U_{L_{2}}$. We represent the bar construction thus obtained by $\hat{\mathrm{B}}\left(\mathfrak{M}_{L_{1}}, \mathfrak{M}_{\emptyset}, \mathfrak{M}_{L_{2}}\right)$. By Proposition 7.35 the maps of monoids and modules determined by the inclusions $C_{L_{1}} \subset U_{L_{1}}$ and $C_{L_{2}} \subset U_{L_{2}}$ induce a homotopy equivalence:

$$
\mathrm{B}\left(\mathfrak{M}_{L_{1}}, \mathfrak{M}_{\emptyset}, \mathfrak{M}_{L_{2}}\right) \stackrel{\simeq}{\rightarrow} \hat{\mathrm{B}}\left(\mathfrak{M}_{L_{1}}, \mathfrak{M}_{\emptyset}, \mathfrak{M}_{L_{2}}\right)
$$

In the proof of Proposition 8.42 we will also need to consider the bar construction induced by the left and right actions of $\mathfrak{M}_{\emptyset}$ on itself defined using the maps $Q_{2}^{\ell}=$ $Q_{2}^{r}=\mathrm{id}: \mathfrak{M}_{\emptyset, 2} \rightarrow \mathfrak{M}_{\emptyset, 2}$ and

$$
\begin{aligned}
Q_{11}^{\ell}: \mathfrak{M}_{\emptyset, 1}^{C_{L_{1}}} \times \mathfrak{M}_{\emptyset, 1}^{U_{L_{2}}} \stackrel{\boxplus_{\emptyset, \emptyset}^{\longrightarrow}}{\longrightarrow} \mathfrak{M}_{\emptyset, 2} & Q_{1}^{\ell}: \mathfrak{M}_{\emptyset, 1}^{C_{L_{2}}} \stackrel{i}{\rightarrow} \mathfrak{M}_{\emptyset, 1}^{U_{L_{2}}} \\
Q_{11}^{r}: \mathfrak{M}_{\emptyset, 1}^{U_{L_{1}}} \times \mathfrak{M}_{\emptyset, 1}^{C_{L_{2}}} \stackrel{\boxplus_{\emptyset, \emptyset}}{\longrightarrow} \mathfrak{M}_{\emptyset, 2} & Q_{1}^{r}: \mathfrak{M}_{\emptyset, 1}^{C_{L_{1}}} \stackrel{i}{\rightarrow} \mathfrak{M}_{\emptyset, 1}^{U_{L_{1}}}
\end{aligned}
$$

(compare with equations (8.31) and (8.32) ). We represent the bar construction thus obtained by

$$
B_{\emptyset, 2}=\mathrm{B}\left(\mathfrak{M}_{\emptyset}, \mathfrak{M}_{\emptyset}, \mathfrak{M}_{\emptyset}\right)_{2}
$$

The maps $W_{M}=W_{N}=$ id: $\mathfrak{M}_{\emptyset, 2} \rightarrow \mathfrak{M}_{\emptyset, 2}$ and

$$
W_{11}: \mathfrak{M}_{\emptyset, 1}^{U_{L_{1}}} \times \mathfrak{M}_{\emptyset, 1}^{U_{L_{2}}} \stackrel{\boxplus_{\emptyset, \emptyset}}{\longrightarrow} \mathfrak{M}_{\emptyset, 2}
$$

induce a map $f: B_{\emptyset, 2} \rightarrow \mathfrak{M}_{\emptyset, 2}$.

Proposition 8.36. The map $f: B_{\emptyset, 2} \rightarrow \mathfrak{M}_{\emptyset, 2}$ is a homotopy equivalence.

Proof. If we let $\hat{B}_{\emptyset, 2}$ denote the bar construction obtained by taking $C_{L_{1}}=U_{L_{1}}$ and $C_{L_{2}}=U_{L_{2}}$ (see remark 8.34), the inclusion map $B_{\emptyset, 2} \rightarrow \hat{B}_{\emptyset, 2}$ is a homotopy equivalence and the map $f$ is the composition $B_{\emptyset, 2} \stackrel{\simeq}{\longrightarrow} \hat{B}_{\emptyset, 2} \rightarrow \mathfrak{M}_{\emptyset, 2}$, which is a homotopy equivalence (Proposition 2.4).

8.5. The space $\mathbf{B}\left(\left|\boldsymbol{B}_{\boldsymbol{J}}\right|, \mathfrak{M}_{\emptyset},\left|\boldsymbol{B}_{\boldsymbol{K}}\right|\right)$ in degree 2. Consider the monoid structure on $\mathfrak{M}_{\emptyset, \leq 2}$ defined in equation (8.30). We define a left action in degrees up to 2 of the monoid $\mathfrak{M}_{\emptyset, \leq 2}$, seen as a constant simplicial space, on the graded simplicial space $B_{L_{2}}=\star \amalg B_{L_{2}, 1} \amalg B_{L_{2}, 2} \amalg \star \amalg \cdots$ (see equations (8.25) and (8.10) ) by the natural transformations:

$$
Q_{1}^{\ell}: \mathfrak{M}_{\emptyset, 1}^{C_{L_{2}}} \stackrel{i}{\rightarrow} \mathfrak{M}_{\emptyset, 1}^{U_{L_{2}}} \subset B_{L_{2}, 1}(n) \quad \text { and } \quad Q_{2}^{\ell}: \mathfrak{M}_{\emptyset, 2} \stackrel{\subset}{\rightarrow} B_{L_{2}, 2}(n)
$$


and by the natural transformation $Q_{11}^{\ell}: \mathfrak{M}_{\emptyset, 1}^{C_{L_{1}}} \times B_{L_{2}, 1} \rightarrow B_{L_{2}, 2}$ defined by

$$
\begin{aligned}
= & \mathfrak{M}_{\emptyset, 1}^{C_{L_{1}}} \times \mathfrak{M}_{\emptyset, 1}^{U_{L_{2}}} \amalg \coprod_{i=1}^{n}\left(\mathfrak{M}_{\emptyset, 1}^{C_{L_{1}}} \times\left(\prod_{x \in L_{2}} \mathfrak{M}_{\emptyset}^{D_{x}}\right)_{1}\right) \amalg \mathfrak{M}_{\emptyset, 1}^{C_{L_{1}}} \times\left(\prod_{x \in L_{2}} \mathfrak{M}_{x}^{D_{x}}\right)_{1} \\
& \stackrel{\boxplus_{\emptyset, \emptyset} \amalg i \amalg i}{\longrightarrow} \mathfrak{M}_{\emptyset, 2} \amalg \coprod_{i=1}^{n}\left(\mathfrak{M}_{\emptyset, 1}^{C} \times\left(\prod_{x \in L_{2}} \mathfrak{M}_{\emptyset}^{D_{x}}\right)_{1}\right) \amalg \mathfrak{M}_{\emptyset, 1}^{C} \times\left(\prod_{x \in L_{2}} \mathfrak{M}_{x}^{D_{x}}\right)_{1} \subset B_{L_{2}, 2}(n)
\end{aligned}
$$

To check that these maps define a natural transformation it is enough to observe that the following diagram is commutative:

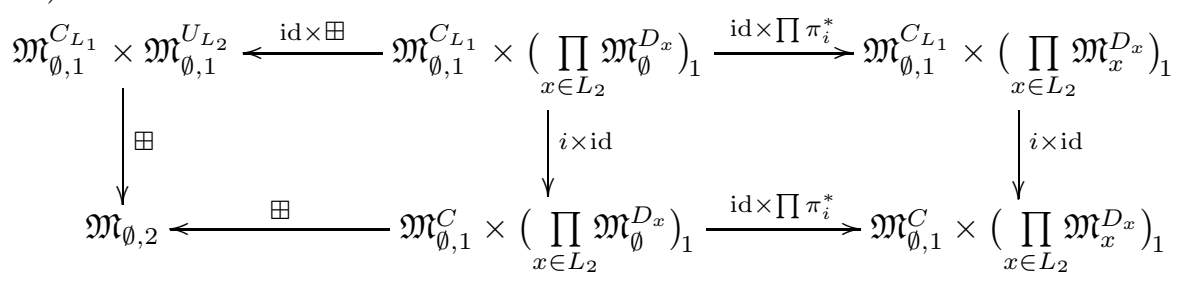

Relation (8.7) follows from the commutativity of the diagram:

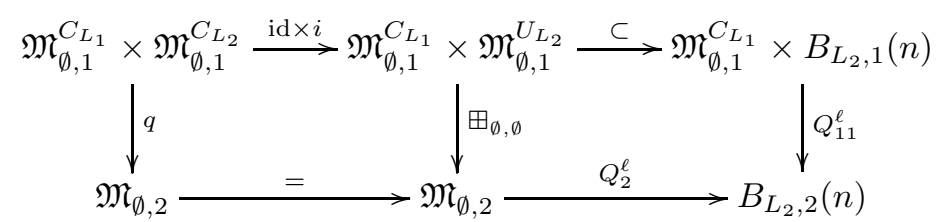

where the map in the top row equals id $\times Q_{1}^{\ell}$.

To define a right action of $\mathfrak{M}_{\emptyset}$ on $\left|B_{L_{1}}\right|$ in degrees up to 2 we proceed as follows: By taking the opposite monoid structure on $\mathfrak{M}_{\emptyset}$, defined using the map $\boxplus_{\emptyset, \emptyset}: \mathfrak{M}_{\emptyset, 1}^{C_{L_{2}}} \times \mathfrak{M}_{\emptyset, 1}^{C_{L_{1}}} \rightarrow \mathfrak{M}_{\emptyset, 2}$, we get, in a standard way, a right action of $\mathfrak{M}_{\emptyset}$ on $B_{L_{2}}$. If we then switch every occurrence of the finite sets $L_{1}$ and $L_{2}$ in the definitions above, we recover the original monoid structure on $\mathfrak{M}_{\emptyset}$ (equation (8.30), together with a right action on $B_{L_{1}}$. Explicitly, we have $Q_{2}^{r}=Q_{2}^{\ell}$, the map $Q_{1}^{r}$ is induced by the inclusion $\mathfrak{M}_{\emptyset, 1}^{C_{L_{1}}} \subset \mathfrak{M}_{\emptyset, 1}^{U_{L_{1}}}$ and the map $Q_{11}^{r}$ is induced by the inclusion $\mathfrak{M}_{\emptyset, 1}^{C_{L_{2}}} \rightarrow \mathfrak{M}_{\emptyset, 1}^{C}$ and by the map

$$
\boxplus_{\emptyset, \emptyset}: \mathfrak{M}_{\emptyset, 1}^{U_{L_{1}}} \times \mathfrak{M}_{\emptyset, 1}^{C_{L_{2}}} \rightarrow \mathfrak{M}_{\emptyset, 2}
$$

(compare with equations (8.37) and (8.38) ). These actions give rise to a bar construction $\mathrm{B}\left(\left|B_{L_{1}}\right|, \mathfrak{M}_{\emptyset},\left|B_{L_{2}}\right|\right)$.

Consider the map $h_{\boxplus}:\left|B_{L_{j}}\right| \rightarrow \mathfrak{M}_{L_{j}}$ (with $j=1,2$ ) defined in degree 1 by composing the map in equation (8.12) with the homeomorphism $\mathfrak{M}_{L_{j}}^{U_{L_{j}}} \cong \mathfrak{M}_{L_{j}}$ and defined in degree 2 in equation (8.24).

Proposition 8.40. The map $h_{\boxplus}$ is equivariant with respect to both the left actions of the monoid $\mathfrak{M}_{\emptyset}$ on $\left|B_{L_{2}}\right|$ and $\mathfrak{M}_{L_{2}}^{U_{L_{2}}}$ (equation (8.31), and the right actions of $\mathfrak{M}_{\emptyset}$ on $\left|B_{L_{1}}\right|$ and $\mathfrak{M}_{L_{1}}^{U_{L_{1}}}$ (equation (8.32) $)$. 
Proof. Consider first the left actions. We need to show that the following diagrams are commutative:
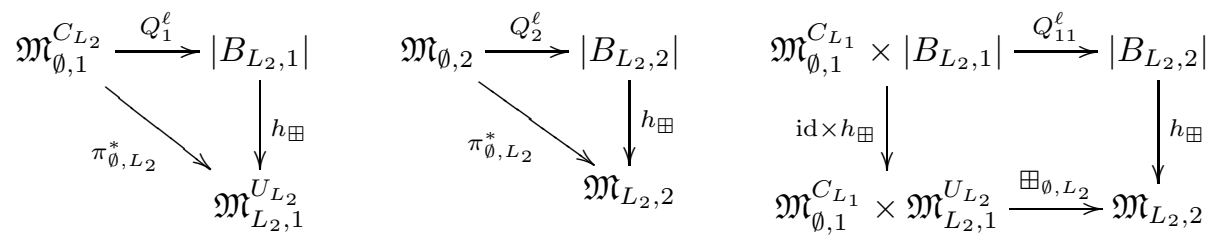

That is clearly the case for the first 2 diagrams. The commutativity of the third diagram amounts to the commutativity of the following diagrams:
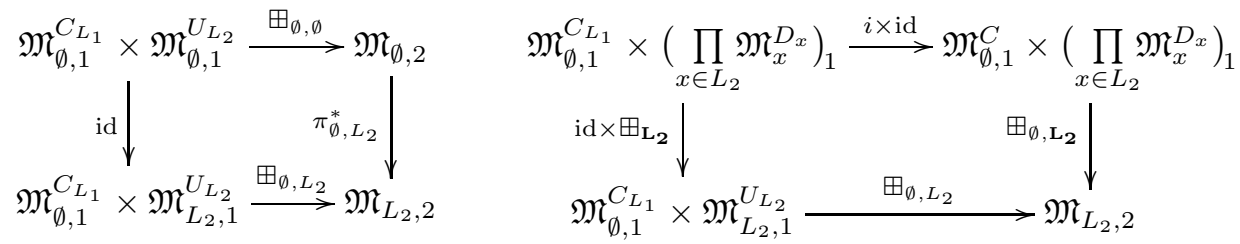

The proof for the right actions is completely analogous.

By Proposition 8.40, the map $h_{\boxplus}$ induces a map in degree 2 :

$$
\mathrm{B}\left(\left|B_{L_{1}}\right|, \mathfrak{M}_{\emptyset},\left|B_{L_{2}}\right|\right) \rightarrow \mathrm{B}\left(\mathfrak{M}_{L_{1}}, \mathfrak{M}_{\emptyset}, \mathfrak{M}_{L_{2}}\right) .
$$

8.6. The Diagram (8.1). In the previous sections we defined, for $k=2$, the bar constructions and all maps except the top one appearing in diagram (8.1) (see equations (8.24), (8.33) and (8.41)).

We now consider the case $k=1$. We take all Jordan open sets to be $\mathbb{C}$ (see Remark 8.13). The maps $h_{\boxplus}$ and $f$ are defined in equations 8.12) and (8.15) respectively. A left action of $\mathfrak{M}_{\emptyset}$ on the simplicial space $B_{L_{2}}$ in degree 1 is defined by the natural transformation $Q_{1}^{\ell}: \mathfrak{M}_{\emptyset, 1} \stackrel{\subset}{\rightarrow} B_{L_{2}, 1}(n)$ and a left action of $\mathfrak{M}_{\emptyset}$ on the space $\mathfrak{M}_{L_{2}}$ in degree 1 is defined by the map $Q_{1}^{\ell}=\pi_{\emptyset, L_{2}}^{*}: \mathfrak{M}_{\emptyset, 1} \rightarrow \mathfrak{M}_{L_{2}, 1}$. The corresponding right actions are defined similarly. The map $h_{\boxplus}$ is easily seen to be equivariant with respect to these actions so it induces a map in degree 1:

$$
\mathrm{B}\left(\left|B_{L_{1}}\right|, \mathfrak{M}_{\emptyset},\left|B_{L_{2}}\right|\right) \rightarrow \mathrm{B}\left(\mathfrak{M}_{L_{1}}, \mathfrak{M}_{\emptyset}, \mathfrak{M}_{L_{2}}\right) .
$$

Proposition 8.42. Let $L_{1}, L_{2} \subset I$ be disjoint finite sets such that $U_{L_{1}} \cap U_{L_{2}}=$ $\emptyset$ and let $L=L_{1} \cup L_{2}$. Then there is a map $\mathrm{B}\left(\left|B_{L_{1}}\right|, \mathfrak{M}_{\emptyset},\left|B_{L_{2}}\right|\right) \rightarrow\left|B_{L}\right|$ in degrees 1 and 2 which is a homotopy equivalence and such that the following diagram commutes up to homotopy:

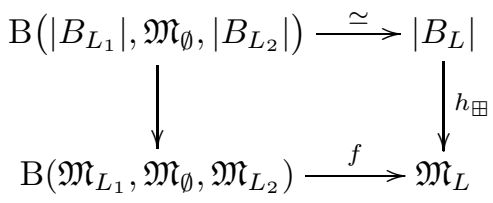

The method of proof of this proposition will be generalized when we prove Proposition 5.12 on page 63 
Proof. Consider first the degree $k=2$ case. We begin by defining a trisimplicial space $F_{0}:(\Delta \times \Delta \times \Delta)^{\mathrm{op}} \rightarrow$ Top homeomorphic to $\mathrm{B} \bullet\left(B_{L_{1}}(\bullet), \mathfrak{M}_{\emptyset}, B_{L_{2}}(\bullet)\right)$. For each non-negative integer $n$ let

$$
A_{2}(n)=\left(\coprod_{i=1}^{n} \mathfrak{M}_{\emptyset, 2}\right) \amalg\left(\coprod_{\substack{i, j=1 \\ i<j}}^{n} \mathfrak{M}_{\emptyset, 1}^{C_{L_{1}}} \times \mathfrak{M}_{\emptyset, 1}^{C_{L_{2}}}\right), \quad A_{L_{j}, 1}(n)=\coprod_{i=1}^{n} \mathfrak{M}_{\emptyset, 1}^{C_{L_{j}}} \quad(j=1,2) .
$$

Note that $A_{2}(n)$ and $A_{L_{j}, 1}(n)$ are homeomorphic respectively to the degree 1 and 2 summands of $\left(\mathfrak{M}_{\emptyset}\right)^{n}$. Then let

$$
\begin{aligned}
& F_{0}\left(n_{1}, n, n_{2}\right)=B_{L_{1}, 2}\left(n_{1}\right) \amalg A_{2}(n) \amalg B_{L_{2}, 2}\left(n_{2}\right) \\
& \quad \amalg B_{L_{1}, 1}\left(n_{1}\right) \times A_{L_{2}, 1}(n) \amalg B_{L_{1}, 1}\left(n_{1}\right) \times B_{L_{2}, 1}\left(n_{2}\right) \amalg A_{L_{1}, 1}(n) \times B_{L_{2}, 1}\left(n_{2}\right) .
\end{aligned}
$$

The homeomorphisms $\psi_{U}$ induce homeomorphisms

$$
F_{0}\left(n_{1}, n, n_{2}\right) \cong \mathrm{B}_{n}\left(B_{L_{1}}\left(n_{1}\right), \mathfrak{M}_{\emptyset}, B_{L_{2}}\left(n_{2}\right)\right)
$$

which give $F_{0}$ the structure of a trisimplicial space. Realizing $F_{0}$ with respect to $n_{1}$ and $n_{2}$ we obtain the simplicial space $\mathrm{B} \bullet\left(\left|B_{L_{1}}\right|, \mathfrak{M}_{\emptyset},\left|B_{L_{2}}\right|\right)$.

Let $F_{1}$ be the bisimplicial space obtained by realizing $F_{0}$ with respect to $n$. We write $\left|F_{1}\right|$ for its geometric realization, which may be described in 3 homeomorphic ways (see [39, Lemma on page 94]): realizing with respect to one of the $n_{i}$ 's and then with respect to the other, or realizing the diagonal simplicial space (if we let $d: \Delta \rightarrow \Delta \times \Delta$ be the diagonal functor, then $\left.\left|d^{*} F_{1}\right| \cong\left|F_{1}\right|\right)$. Let

$$
B_{\emptyset, 1}^{L_{1}}=\mathrm{B}\left(\mathfrak{M}_{\emptyset}^{U_{L_{1}}}, \mathfrak{M}_{\emptyset}^{C_{L_{1}}}, \mathfrak{M}_{\emptyset}^{C}\right)_{1}, \quad B_{\emptyset, 1}^{L_{2}}=\mathrm{B}\left(\mathfrak{M}_{\emptyset}^{C}, \mathfrak{M}_{\emptyset}^{C_{L_{2}}}, \mathfrak{M}_{\emptyset}^{U_{L_{2}}}\right)_{1}
$$

(see Proposition 8.16) and let $B_{\emptyset, 2}$ be as in equation (8.35). Also recall Notation 8.11. All the face maps in $d^{*} F_{1}$ between non-degenerate summands are represented in the diagram below (in the lower half of the diagram the order of the 
factors in some of the products was switched to make the maps clearer):

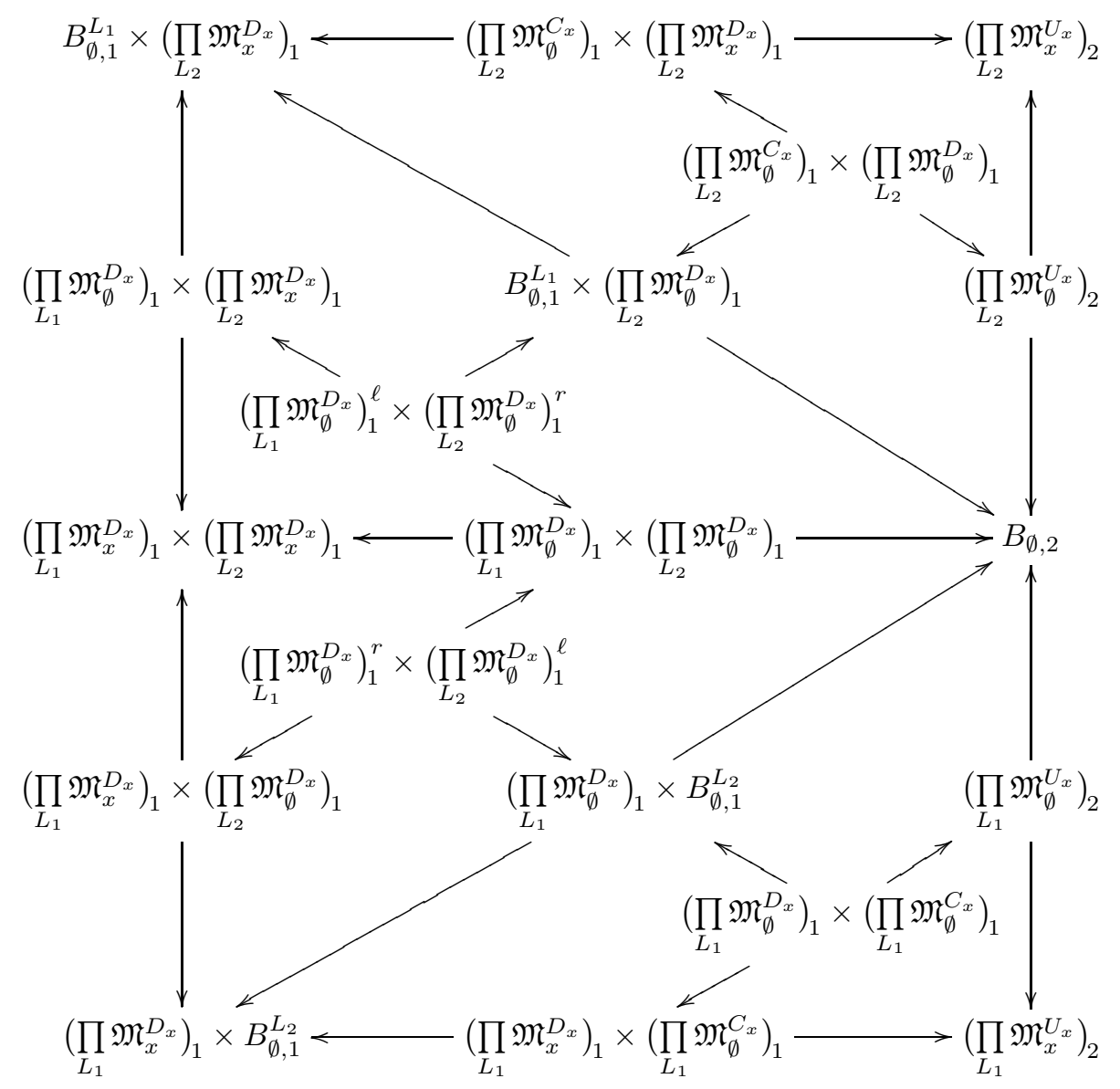

We now describe a weak equivalence $d^{*} F_{1} \rightarrow B_{L, 2}^{\text {big }}$ (see diagram (8.28)). We only need to define it on $d^{*} F_{1}(n)$ for $n \leq 2$ since all summands in $d^{*} F_{1}(n)$ are degenerate for $n>2$. The map $d^{*} F_{1}(0) \rightarrow B_{L, 2}^{\mathrm{big}}(0)$ is defined by the homotopy equivalence $B_{\emptyset, 2} \rightarrow \mathfrak{M}_{\emptyset, 2}$ of Proposition 8.36 , by the map

$$
\begin{aligned}
& \left(\prod_{x \in L_{1}} \mathfrak{M}_{x}^{U_{x}}\right)_{2} \amalg\left(\prod_{x \in L_{2}} \mathfrak{M}_{x}^{U_{x}}\right)_{2} \amalg\left(\prod_{x \in L_{1}} \mathfrak{M}_{x}^{D_{x}}\right)_{1} \times\left(\prod_{y \in L_{2}} \mathfrak{M}_{x}^{D_{x}}\right)_{1} \\
& \stackrel{D_{x} \subset U_{x}}{\longrightarrow}\left(\prod_{x \in L_{1}} \mathfrak{M}_{x}^{U_{x}}\right)_{2} \amalg\left(\prod_{x \in L_{2}} \mathfrak{M}_{x}^{U_{x}}\right)_{2} \amalg\left(\prod_{x \in L_{1}} \mathfrak{M}_{x}^{U_{x}}\right)_{1} \times\left(\prod_{x \in L_{2}} \mathfrak{M}_{x}^{U_{x}}\right)_{1}=\left(\prod_{x \in L} \mathfrak{M}_{x}^{U_{x}}\right)_{2}
\end{aligned}
$$

and by the map

$$
\begin{aligned}
& \left(\prod_{x \in L_{1}} \mathfrak{M}_{x}^{D_{x}}\right)_{1} \times B_{\emptyset, 1}^{L_{2}} \amalg B_{\emptyset, 1}^{L_{1}} \times\left(\prod_{x \in L_{2}} \mathfrak{M}_{x}^{D_{x}}\right)_{1} \\
& \quad \cong\left(\coprod_{x \in L_{1}} B_{\emptyset, 1}^{L_{2}} \times \mathfrak{M}_{x, 1}^{D_{x}}\right) \amalg\left(\coprod_{x \in L_{2}} B_{\emptyset, 1}^{L_{1}} \times \mathfrak{M}_{x, 1}^{D_{x}}\right) \stackrel{f \times \mathrm{id}}{\longrightarrow} \coprod_{x \in L} \mathfrak{M}_{\emptyset, 1}^{B_{x}} \times \mathfrak{M}_{x, 1}^{D_{x}}
\end{aligned}
$$

where $f$ is the homotopy equivalence from Proposition 8.16. which is well defined since $U_{L_{2}} \subset B_{x}$ for $x \in L_{1}$ and $U_{L_{1}} \subset B_{x}$ for $x \in L_{2}$. 
The map $d^{*} F_{1}(1) \rightarrow B_{L, 2}^{\mathrm{big}}(1)$ is defined on non-degenerate summands by the map

$$
\left(\prod_{x \in L_{1}} \mathfrak{M}_{\emptyset}^{U_{x}}\right)_{2} \amalg\left(\prod_{x \in L_{2}} \mathfrak{M}_{\emptyset}^{U_{x}}\right)_{2} \amalg\left(\prod_{x \in L_{1}} \mathfrak{M}_{\emptyset}^{D_{x}}\right)_{1} \times\left(\prod_{y \in L_{2}} \mathfrak{M}_{\emptyset}^{D_{x}}\right)_{1} \rightarrow\left(\prod_{x \in L} \mathfrak{M}_{\emptyset}^{U_{x}}\right)_{2}
$$

(compare with equation (8.44)), the map

$$
\left(\prod_{x \in L_{1}} \mathfrak{M}_{\emptyset}^{D_{x}}\right)_{1} \times B_{\emptyset, 1}^{L_{2}} \amalg B_{\emptyset, 1}^{L_{1}} \times\left(\prod_{x \in L_{2}} \mathfrak{M}_{\emptyset}^{D_{x}}\right)_{1} \rightarrow \coprod_{x \in L} \mathfrak{M}_{\emptyset, 1}^{B_{x}} \times \mathfrak{M}_{\emptyset, 1}^{D_{x}}
$$

(compare with equation (8.45) $)$, the map

$$
\begin{aligned}
& \left(\prod_{x \in L_{1}} \mathfrak{M}_{x}^{D_{x}}\right)_{1} \times\left(\prod_{x \in L_{1}} \mathfrak{M}_{\emptyset}^{C_{x}}\right)_{1} \amalg\left(\prod_{x \in L_{2}} \mathfrak{M}_{\emptyset}^{C_{x}}\right)_{1} \times\left(\prod_{x \in L_{2}} \mathfrak{M}_{x}^{D_{x}}\right)_{1}
\end{aligned}
$$

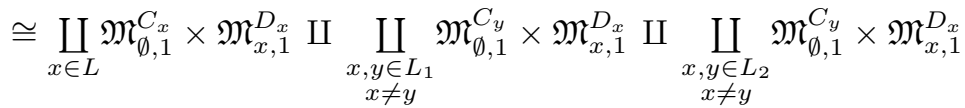

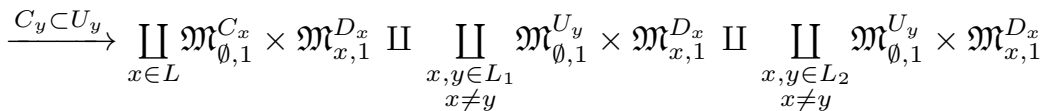

and the map

$$
\begin{aligned}
& \left(\prod_{x \in L_{1}} \mathfrak{M}_{x}^{D_{x}}\right)_{1} \times\left(\prod_{x \in L_{2}} \mathfrak{M}_{\emptyset}^{D_{x}}\right)_{1} \amalg\left(\prod_{x \in L_{1}} \mathfrak{M}_{\emptyset}^{D_{x}}\right)_{1} \times\left(\prod_{x \in L_{2}} \mathfrak{M}_{x}^{D_{x}}\right)_{1} \\
& \cong \coprod_{\substack{x \in L_{1} \\
y \in L_{2}}} \mathfrak{M}_{\emptyset, 1}^{D_{y}} \times \mathfrak{M}_{x, 1}^{D_{x}} \amalg \coprod_{\substack{y \in L_{1} \\
x \in L_{2}}} \mathfrak{M}_{\emptyset, 1}^{D_{y}} \times \mathfrak{M}_{x, 1}^{D_{x}}
\end{aligned}
$$

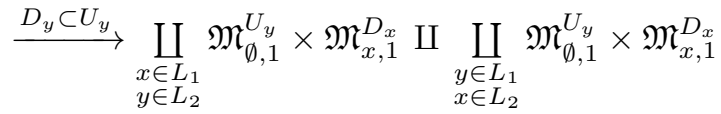

The map $d^{*} F_{1}(2) \rightarrow B_{L, 2}^{\text {big }}(2)$ is defined on non-degenerate summands by the map

$$
\begin{aligned}
& \left(\prod_{x \in L_{1}} \mathfrak{M}_{\emptyset}^{D_{x}}\right)_{1} \times\left(\prod_{x \in L_{1}} \mathfrak{M}_{\emptyset}^{C_{x}}\right)_{1} \amalg\left(\prod_{x \in L_{2}} \mathfrak{M}_{\emptyset}^{C_{x}}\right)_{1} \times\left(\prod_{x \in L_{2}} \mathfrak{M}_{\emptyset}^{D_{x}}\right)_{1}
\end{aligned}
$$

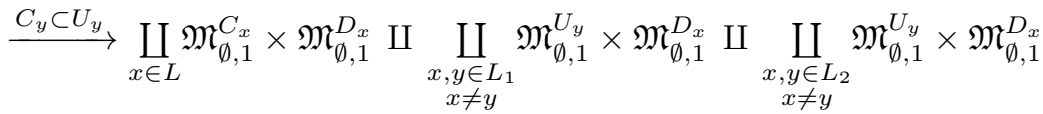

and the map

$$
\begin{aligned}
\left(\prod_{x \in L_{1}} \mathfrak{M}_{\emptyset}^{D_{x}}\right)_{1}^{r} \times\left(\prod_{x \in L_{2}} \mathfrak{M}_{\emptyset}^{D_{x}}\right)_{1}^{\ell} \amalg\left(\prod_{x \in L_{1}} \mathfrak{M}_{\emptyset}^{D_{x}}\right)_{1}^{\ell} \times\left(\prod_{x \in L_{2}} \mathfrak{M}_{\emptyset}^{D_{x}}\right)_{1}^{r} & \cong \coprod_{\substack{x \in L_{1} \\
y \in L_{2}}} \mathfrak{M}_{\emptyset, 1}^{D_{y}, \ell} \times \mathfrak{M}_{\emptyset, 1}^{D_{x}, r} \amalg \coprod_{\substack{y \in L_{1} \\
x \in L_{2}}} \mathfrak{M}_{\emptyset, 1}^{D_{y}, \ell} \times \mathfrak{M}_{\emptyset, 1}^{D_{x}, r} \\
& \stackrel{D_{y} \subset U_{y}}{\longrightarrow} \underset{\substack{x \in L_{1} \\
y \in L_{2}}}{\coprod_{\emptyset, 1}} \mathfrak{M}_{U_{y}}^{U_{y}} \times \mathfrak{M}_{\emptyset, 1}^{D_{x}} \amalg \coprod_{\substack{y \in L_{1} \\
x \in L_{2}}} \mathfrak{M}_{\emptyset, 1}^{U_{y}} \times \mathfrak{M}_{\emptyset, 1}^{D_{x}}
\end{aligned}
$$

In this way we get a diagram:

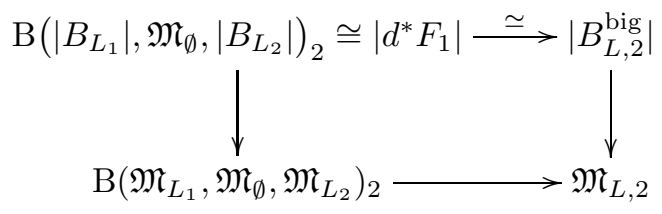


which commutes by the commutativity and associativity of the gluing maps (Proposition (7.46). This finishes the proof for $k=2$. The proof for $k=1$ uses analogous but much simpler arguments.

8.7. Proof of Theorem 1.1, We can now prove Theorem 1.1, which we restate here. Let $\mathbb{V}$ be a finite dimensional complex Hermitian vector space and denote the dependence of the bar construction on $\mathbb{V}$ by $B_{L}^{\mathbb{V}}$.

Theorem 1.1, Let $I=\left\{x_{1}, \ldots, x_{q}\right\} \subset \mathbb{C}^{2}$. Then:

(1) The map $h_{\boxplus}:\left|B_{I}^{\mathbb{V}}\right| \rightarrow \mathfrak{M}_{I}^{\mathbb{V}}$ from equations (8.12) and (8.24) is a homotopy equivalence in the $k=1,2$ components.

(2) If $I=J \cup K$, with $J \cap K=\emptyset$, then the map $\mathrm{B}\left(\mathfrak{M}_{J}^{\mathbb{V}}, \mathfrak{M}_{\emptyset}^{\mathbb{V}}, \mathfrak{M}_{K}^{\mathbb{V}}\right) \rightarrow \mathfrak{M}_{I}^{\mathbb{V}}$ from equations (8.15) and (8.33) is a homotopy equivalence in the $k=1,2$ components.

Proof. From Proposition 8.42 with $L_{1}=J$ and $L_{2}=K$ it follows that part (2) of the theorem is a consequence of part (1), which we now prove. Let $\mathfrak{C}$ be as in Definition 3.9 and let $\mathfrak{C}_{I, k}$ be as in Definition 6.1. Let $\mathfrak{M}_{k}^{\mathbb{V}}: \mathfrak{C} \rightarrow$ Top be the functor defined on objects by $\mathfrak{M}_{k}^{\mathbb{V}}(I)=\mathfrak{M}_{I, k}^{\mathbb{V}}$ and defined on morphisms by pullback. Also, let $B_{k}^{\mathbb{V}}: \mathfrak{C} \times \Delta^{\text {op }} \rightarrow \underline{\text { Top }}$ be the functor defined on objects by $B_{k}^{\mathbb{V}}(n, J)=B_{J, k}^{\mathbb{V}}(n)$. Analogous but simpler arguments to those in the proof of Theorem 1.3 (on page 19) show that we have a commutative diagram

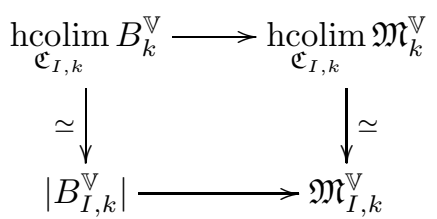

where the vertical maps are homotopy equivalences, so the proof will be complete if we show that the top horizontal map is a homotopy equivalence. Thus, for $k=1$ we only need to consider the trivial case when $\# I=1$, and for $k=2$, we only need to consider the case where $I=\{x, y\} \subset \mathbb{C}^{2}$ which we now analyze. Now consider Proposition 8.42 in the case where $L_{1}=\{x\}, L_{2}=\{y\}$ and $L=\{x, y\}$. The maps $\left|B_{x}^{\mathbb{V}}\right| \rightarrow \mathfrak{M}_{x}^{\mathbb{V}}$ in diagram (8.43) are trivially homotopy equivalences hence the left vertical map is a homotopy equivalence. Thus we only have to show that the bottom horizontal map in diagram (8.43) is a homotopy equivalence. Consider the bar construction in Remark 8.34. We have maps

$$
\mathrm{B}\left(\mathfrak{M}_{x}^{\mathbb{V}}, \mathfrak{M}_{\emptyset}^{\mathbb{V}}, \mathfrak{M}_{y}^{\mathbb{V}}\right) \stackrel{\simeq}{\rightarrow} \hat{\mathrm{B}}\left(\mathfrak{M}_{x}^{\mathbb{V}}, \mathfrak{M}_{\emptyset}^{\mathbb{V}}, \mathfrak{M}_{y}^{\mathbb{V}}\right) \rightarrow \mathfrak{M}_{L}^{\mathbb{V}}
$$

The face maps between the non-degenerate degree 2 summands in $\hat{\mathrm{B}}_{\bullet}\left(\mathfrak{M}_{x}^{\mathbb{V}}, \mathfrak{M}_{\emptyset}^{\mathbb{V}}, \mathfrak{M}_{y}^{\mathbb{V}}\right)$ as well as the map $\hat{\mathrm{B}}_{0}\left(\mathfrak{M}_{x}^{\mathbb{V}}, \mathfrak{M}_{\emptyset}^{\mathbb{V}}, \mathfrak{M}_{y}^{\mathbb{V}}\right) \rightarrow \mathfrak{M}_{L}^{\mathbb{V}}$ are represented in the following cube 
diagram (where we omit $\mathbb{V}$ from the notation):

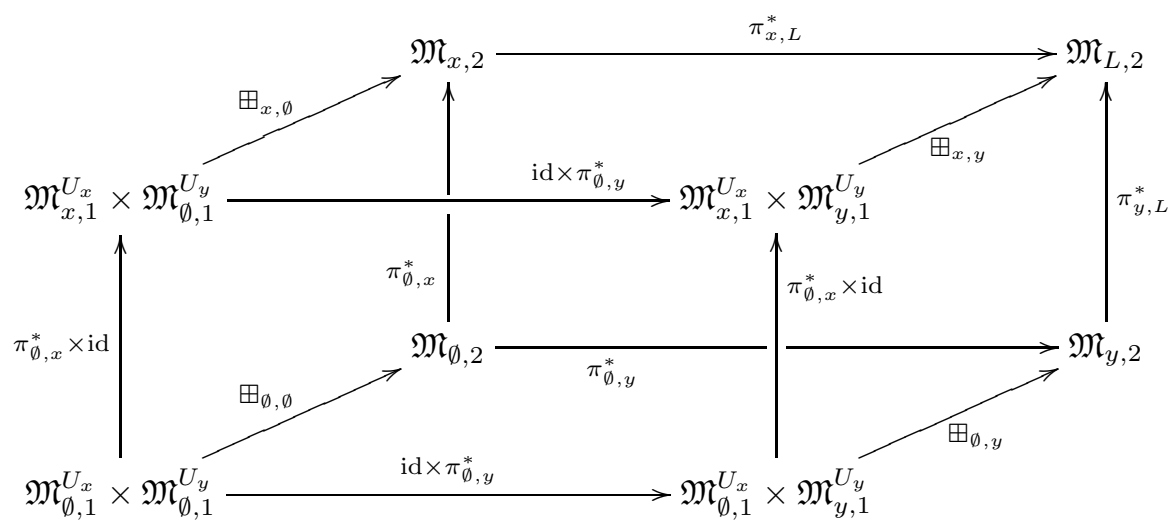

The diagram obtained from the cube by removing the vertex $\mathfrak{M}_{L, 2}^{\mathbb{V}}$ is homeomorphic to the nerve $N$ of the open cover of $\mathfrak{M}_{L, 2}^{\mathbb{V}}$ in Corollary 7.45 (see Example 2.1 for the definition of nerve), and the barycentric subdivision of $\hat{\mathrm{B}} \cdot\left(\mathfrak{M}_{x}^{\mathbb{V}}, \mathfrak{M}_{\emptyset}^{\mathbb{V}}, \mathfrak{M}_{y}^{\mathbb{V}}\right.$ ) (see [38, section 8.3.A, page 60]) is homeomorphic to $B_{\bullet}(*, N, *)$. It follows from [43, Proposition 4.1] that the map $\hat{\mathrm{B}}\left(\mathfrak{M}_{x}^{\mathbb{V}}, \mathfrak{M}_{\emptyset}^{\mathbb{V}}, \mathfrak{M}_{y}^{\mathbb{V}}\right) \rightarrow \mathfrak{M}_{L}^{\mathbb{V}}$ is a homotopy equivalence, which concludes the proof of the theorem.

Taking the limit when $\operatorname{dim} \mathbb{V} \rightarrow \infty$ we can now prove:

Theorem 8.47. Let $\mathbb{H}$ be a countably infinite dimensional complex Hermitian vector space. Then the map $h_{I}:\left\|\mathfrak{B}_{I}^{\mathbb{H}}\right\| \rightarrow \mathfrak{M}_{I}^{\mathbb{H}}$ in Definition 5.2 is a homotopy equivalence in degrees 1 and 2.

Proof. The maps in Proposition 7.46 pass to the colimit to define maps $\boxplus_{\mathbf{I}}: \prod_{i} \mathfrak{M}_{I_{i}}^{\mathbb{H}, U_{i}} \rightarrow$ $\mathfrak{M}_{I}^{\mathbb{H}, U}$. We need to see how these maps are related with the action of the linear isometries operad $\mathscr{L}^{\mathbb{H}}$. Let $\imath_{\alpha}: \mathbb{H} \rightarrow \mathbb{H}^{n}$ be inclusion onto the $\alpha$-th factor. Let $\mathcal{P}$ be the suboperad of the endomorphism operad of $\mathbb{H}$ consisting of complex linear maps $f: \mathbb{H}^{n} \rightarrow \mathbb{H}$ such that $f \circ \imath_{\alpha}$ is an isometry for all $\alpha=1, \ldots, n$. Then $\mathcal{P}(n) \cong \mathscr{L}(1)^{n}$ so $\mathcal{P}(n)$ is contractible. Also note that $\mathcal{P}$ contains the linear isometries operad $\mathscr{L}$. We fix basepoints $* \in \mathcal{P}(n)$ given in matrix notation by

$$
*=\left[\begin{array}{lll}
1 & \cdots & 1
\end{array}\right]: \mathbb{H}^{n} \rightarrow \mathbb{H} .
$$

For any $f \in \mathcal{P}(n)$ and each $\alpha=1, \ldots, n$, let $f_{\alpha}=f \circ \imath_{\alpha}$. Given finite disjoint sets $J_{1}, \ldots, J_{n}$ we define a map $\boxplus_{\mathbf{J}, f}$ as the composition:

$$
\boxplus_{\mathbf{J}, f}: \prod_{\alpha=1}^{n} \mathfrak{M}_{J_{\alpha}, k_{\alpha}}^{\mathbb{H}, U_{\alpha}} \stackrel{\prod f_{\alpha}}{\longrightarrow} \prod_{\alpha=1}^{n} \mathfrak{M}_{J_{\alpha}, k_{\alpha}}^{\mathbb{H}, U_{\alpha}} \stackrel{\boxplus_{\mathrm{J}}}{\longrightarrow} \mathfrak{M}_{J, k}^{\mathbb{H}, U} \quad\left(k=\sum k_{\alpha} \leq 2\right) .
$$

Note that, for $f=*$, we have $\boxplus_{\mathbf{J}, f}=\boxplus_{\mathbf{J}}$. The maps $\boxplus_{\mathbf{J}, f}$ are compatible with the operad data in the following sense: Let $j_{1}, \ldots, j_{n}$ be non-negative integers and let $j=\sum j_{i}$; let $f \in \mathcal{P}(n)$ and $g_{i} \in \mathcal{P}\left(j_{i}\right)$; consider finite disjoint sets $J_{\alpha}$, with $\alpha=1, \ldots, j$ and for each $i=1, \ldots, n$, let $s_{i}=j_{1}+\cdots+j_{i}$ and let:

$$
\mathbf{J}_{i}=\left(J_{s_{i-1}+1}, \ldots, J_{s_{i}}\right), \quad K_{i}=\bigcup_{\alpha=s_{i-1}+1}^{s_{i}} J_{\alpha} \quad(i=1, \ldots, n) ;
$$


also, let:

$$
\mathbf{J}=\left(J_{1}, \ldots, J_{j}\right), \quad \mathbf{K}=\left(K_{1}, \ldots, K_{n}\right), \quad J=\bigcup_{i=1}^{n} K_{i}=\bigcup_{\alpha=1}^{j} J_{\alpha} .
$$

Then we have:

$$
\boxplus_{\mathbf{J}, f \circ \prod g_{i}}=\boxplus_{\mathbf{K}, f} \circ \prod_{i=1}^{n} \boxplus_{\mathbf{J}_{i}, g_{i}} .
$$

To prove this last statement, let $\imath_{\alpha}: \mathbb{H} \rightarrow \mathbb{H}^{j_{i}}$ (with $\alpha=1, \ldots, j$ ) be inclusion into the $\left(\alpha-s_{i-1}\right)$-th factor and let $f_{i}=f \circ \imath_{i}$ and $g_{i \alpha}=g_{i} \circ \imath_{\alpha}$. Equation (8.49) then follows from the commutativity of the following diagram (the lower triangle is commutative by Proposition 7.46(2) and the upper right triangle is commutative by Proposition 7.46(5)):

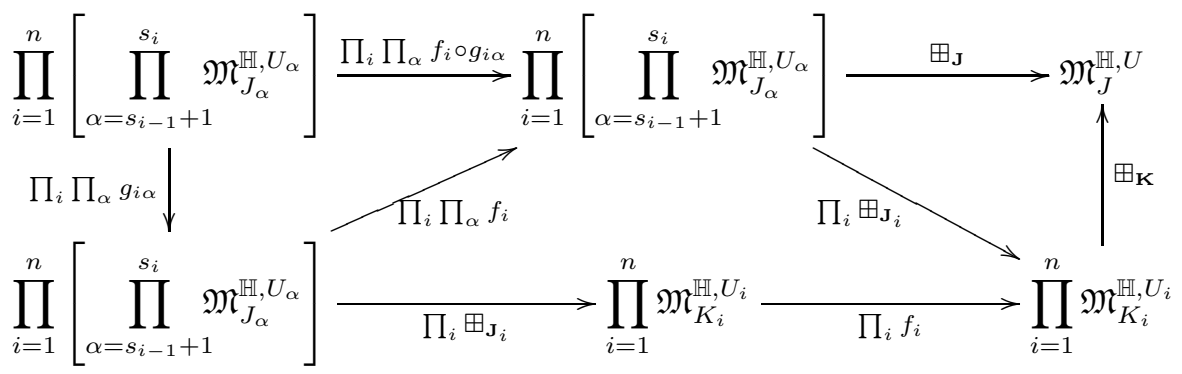

Let $q=\# I$, let $\mathcal{P}_{I}(n)=\mathcal{P}(1+n q)$ and $\mathcal{P}^{I}(n)=\prod_{I} \mathcal{P}(n)$ (compare with Definition 5.1). Consider the category $\Delta\left(\mathcal{P}_{I}, \mathcal{P}^{I}\right)$ (see Definition 4.8). Then we can define a functor $\mathcal{F}_{I}: \Delta\left(\mathcal{P}_{I}, \mathcal{P}^{I}\right)^{\text {op }} \rightarrow$ Top by letting $\mathcal{F}_{I}(n)=B_{I}^{\mathbb{H}}(n)$ (see equation (8.25)) and defining the morphisms using the maps $\boxplus_{\mathbf{J}, f}$ in equation (8.48). The inclusion of operads $\mathscr{L}^{\mathbb{H}} \subset \mathcal{P}$ induces an equivalence of categories $\imath: \Delta_{I} \rightarrow \Delta\left(\mathcal{P}_{I}, \mathcal{P}^{I}\right)$. For $f \in \mathscr{L}^{\mathbb{H}}(n) \subset \mathcal{P}(n)$ we have the commutative diagram (see Proposition 7.46):

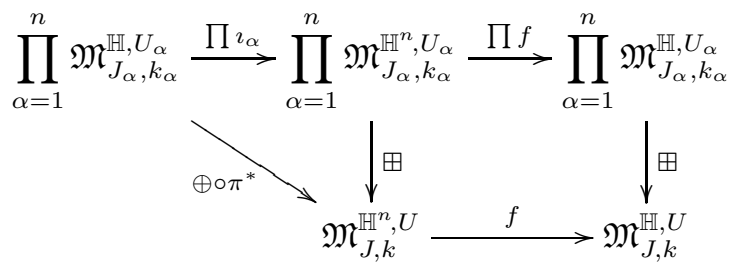

so the inclusions $\mathfrak{M}_{J}^{\mathbb{H}, U} \rightarrow \mathfrak{M}_{J}^{\mathbb{H}}$ induce a weak equivalence from the restriction of $\mathcal{F}_{I}$ to $\Delta_{I}$ to $\mathfrak{B}_{I}^{\mathbb{H}}$. To finish the proof we observe that the inclusion of the base point in $\mathcal{P}(n)$ induces an equivalence of categories $\Delta \rightarrow \Delta\left(\mathcal{P}_{I}, \mathcal{P}^{I}\right)$ and the restriction of $\mathcal{F}_{I}$ to $\Delta$ equals the simplicial space $B_{I}^{\mathbb{H I}}$. The functor $\mathcal{F}_{I}$ extends to a functor $\widetilde{\mathcal{F}}_{I}: \widetilde{\Delta}\left(\mathcal{P}_{I}, \mathcal{P}^{I}\right) \rightarrow \underline{\text { Top }}$ (see Definition 4.13) and $B_{I}^{\mathbb{H}}$ extends to a functor $\widetilde{B}_{I}^{\mathbb{H}}: \widetilde{\Delta} \rightarrow \underline{\text { Top }}$ by letting $\widetilde{\mathcal{F}_{I}(-1)}=B_{I}^{\mathbb{H}}(-1)=\mathfrak{M}_{I}^{\mathbb{H}}$ and defining the functors on 
morphisms in the obvious way, and we have a commutative diagram

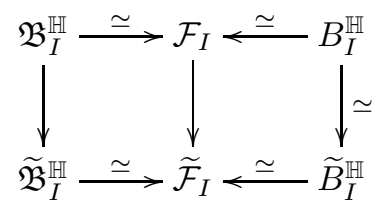

which completes the proof.

\section{The Limit WHEN $k \rightarrow \infty$}

We now consider the limit when $k \rightarrow \infty$. We will work with the moduli space of instantons, which we now describe. For each finite set $J \subset \mathbb{C}^{2}$ let $\#_{J} \overline{\mathbb{P}^{2}}$ be the smooth 4-manifold obtained by collapsing $L_{\infty} \subset \tilde{\mathbb{P}}_{J}^{2}$ to a point $y_{\infty} \in \#_{J} \overline{\mathbb{P}^{2}}$ and let $\bar{\pi}: \tilde{\mathbb{P}}_{J}^{2} \rightarrow \#_{J} \overline{\mathbb{P}^{2}}$ be the collapsing map. Let $g$ be a smooth metric on $\#_{J} \overline{\mathbb{P}}^{2}$ such that $\bar{\pi}^{*} g$ is compatible with the complex structure on $\tilde{\mathbb{P}}_{J}^{2}$. Let $\mathbb{V}$ be a finite dimensional complex Hermitian vector space, let $E \rightarrow \#_{J} \overline{\mathbb{P}^{2}}$ be an $S U(\mathbb{V})$ vector bundle with second Chern class $c_{2}(E)=k$, let $\mathcal{A}(E)$ denote the space of smooth connections on $E$ and let $P_{\infty}$ denote the space of isomorphisms $\phi: E_{y_{\infty}} \rightarrow \mathbb{V}$ equivariant with respect to the $S U(\mathbb{V})$ action. The gauge group $\mathcal{G}$ of smooth automorphisms of $E$ acts freely on $P_{\infty} \times \mathcal{A}(E)$ and we represent the quotient by $\mathscr{C}_{J, k}^{\mathbb{V}}$. The moduli space of instantons is the subspace $\mathfrak{M} \mathcal{I}_{J, k}^{\mathbb{V}} \subset \mathscr{C}_{J, k}^{\mathbb{V}}$ of equivalence classes of connections whose curvature is self-dual with respect to the Hodge $*$ operator. This space is isomorphic to $\mathfrak{M}_{J, k}^{\mathbb{V}}$ (see [10, Theorem 0.1], [32, Corollary 2.14]). Let $\mathfrak{M} \mathcal{I}_{J}^{\mathbb{V}}=\coprod_{k} \mathfrak{M I}_{J, k}^{\mathbb{V}}$ and $\mathscr{C}_{J}^{\mathbb{V}}=\coprod_{k} \mathscr{C}_{J, k}^{\mathbb{V}}$. The same arguments as the ones in section 3 show that $\mathfrak{M} \mathcal{I}_{J}^{\mathbb{V}}$ and $\mathscr{C}_{J}^{\mathbb{V}}$ are $\mathscr{I}_{*}$-functors and we have:

Proposition 9.1. The inclusion $\mathfrak{M} \mathcal{I}_{J}^{\mathbb{V}} \rightarrow \mathscr{C}_{J}^{\mathbb{V}}$ and the isomorphism $\psi_{\mathbb{V}}: \mathfrak{M} \mathcal{I}_{J}^{\mathbb{V}} \rightarrow$ $\mathfrak{M}_{J}^{\mathbb{V}}$ are maps of $\mathscr{I}_{*}$-functors.

Proof. The result is clear for the inclusion and it follows easily for the map $\psi_{\mathbb{V}}$ from its description: given a self-dual connection $\xi$ on $E$, the $(0,1)$-part of the pullback $\left(\bar{\pi}^{*} \xi\right)^{(0,1)}$ is a holomorphic structure on the pullback bundle $\bar{\pi}^{*} E \rightarrow \tilde{\mathbb{P}}_{J}^{2}$ and an isomorphism $\phi \in P_{\infty}$ induces a holomorphic trivialization $\phi \times \mathrm{id}:\left.\bar{\pi}^{*} E\right|_{L_{\infty}}=E_{y_{\infty}} \times$ $L_{\infty} \rightarrow \mathbb{V} \times L_{\infty}$; then $\psi_{\mathbb{V}}([\phi, \xi])=\left[\left(\pi^{*} \xi\right)^{(0,1)}, \phi \times \mathrm{id}\right] \in \mathfrak{M}_{J, k}^{\mathbb{V}}$ (recall Definition 3.2).

Taking the direct limit when $k \rightarrow \infty$ (see the discussion on page 3 before Theorem [1.5) the inclusion $\mathfrak{M I} \mathcal{I}_{J}^{\mathbb{V}} \subset \mathscr{C}_{J}^{\mathbb{V}}$ induces a weak homotopy equivalence $\mathfrak{M} \mathcal{I}_{J, \infty}^{\mathbb{V}} \stackrel{\simeq}{\rightarrow} \mathscr{C}_{J, \infty}^{\mathbb{V}}\left[48\right.$, Theorem $\left.2^{*}\right]$ and $\mathscr{C}_{J}^{\mathbb{V}}$ is homotopically equivalent to the space of based maps $\operatorname{Map}_{*}\left(\#, \overline{\mathbb{P}^{2}}, B S U(\mathbb{V})\right)$ where $B S U(\mathbb{V})$ is the classifying space of $S U(\mathbb{V})$ (see [13, Theorem 1.3]).

We now prove Theorem 1.5 . Since $\overline{\mathbb{P}^{2}}$ has a CW-complex structure given by attaching a 4-dimensional cell $D^{4}$ to $S^{2}$ via the Hopf map $h: S^{3} \rightarrow S^{2}$, we can view maps from $\overline{\mathbb{P}^{2}}$ as maps from $D^{4}$ whose restriction to the boundary factor through $h$. In an analogous way, the space of maps from $\#_{n} \overline{\mathbb{P}^{2}}$ to $B S U(\mathbb{V})$ is homotopy equivalent to the space of maps from $D^{4}$ to $B S U(\mathbb{V})$ which factor through the map $S^{3} \rightarrow \bigvee_{n} S^{3} \stackrel{\bigvee h}{\longrightarrow} \bigvee_{n} S^{2}$. For the remainder of this section $I$ denotes the unit interval: $I=[0,1] \subset \mathbb{R}$. Note that $S^{3} \cong I^{3} / \partial I^{3}$ and $\bigvee_{n} S^{3}$ is the quotient of $I^{3}$ 
by the subspace of triples $\mathbf{x}=\left(x_{1}, x_{2}, x_{3}\right)$ with either $\mathbf{x} \in \partial I^{3}$ or $x_{1}=i / n$ with $i=0, \ldots, n$.

Definition 9.2. Given a based topological space $(X, *)$ let $\mathcal{M}_{*} X$ represent the space of compactly supported maps $f:(-\infty, 0] \times I^{3} \rightarrow X$ such that $f(t, \mathbf{x})=*$ whenever $\mathbf{x} \in \partial\left(I^{3}\right)$.

(1) We identify, up to homotopy, $\Omega^{4} X$ with the subspace of maps $f \in \mathcal{M}_{*} X$ such that $f(0, \mathbf{x})=*$ for any $\mathbf{x} \in I^{3}$.

(2) Let $H: I^{3} \rightarrow S^{2}$ be the composition of the projection $I^{3} \rightarrow I^{3} / \partial I^{3}$ with the Hopf map. We identify, up to homotopy, $\operatorname{Map}_{*}\left(\overline{\mathbb{P}^{2}}, X\right)$ with the subspace of maps $f \in \mathcal{M}_{*} X$ whose restriction to $0 \times I^{3}$ factors through $H$. Restriction to $0 \times I^{3}$ induces a map $\rho: \operatorname{Map}_{*}\left(\overline{\mathbb{P}^{2}}, X\right) \rightarrow \Omega^{2} X$.

(3) Let $\mathbf{x}=\left(x_{1}, x_{2}, x_{3}\right) \in I^{3}$. We identify, up to homotopy, $\operatorname{Map}_{*}\left(\#_{n} \overline{\mathbb{P}^{2}}, X\right)$ with the subspace of maps $f \in \mathcal{M}_{*} X$ such that:

(a) $f\left(0, i / n, x_{2}, x_{3}\right)=*$ for $i=0, \ldots, n$ and any $x_{2}, x_{3} \in I$;

(b) for each $i=1, \ldots, n$, the restriction of $f$ to $0 \times[(i-1) / n, i / n] \times I^{2} \cong I^{3}$ factors through $H: I^{3} \rightarrow S^{2}$.

We have a map $\boldsymbol{\rho}=\left(\rho_{1}, \ldots, \rho_{n}\right): \operatorname{Map}_{*}\left(\#_{n} \overline{\mathbb{P}^{2}}, X\right) \rightarrow\left(\Omega^{2} X\right)^{n}$ whose components $\rho_{i}$ are induced by restriction to $0 \times[(i-1) / n, i / n] \times I^{2}$, for $i=1, \ldots, n$.

We now give $\left(\Omega^{4} X\right)^{n}$ the structure of an associative monoid and define actions of $\left(\Omega^{4} X\right)^{n}$ on $\left(\operatorname{Map}_{*}\left(\overline{\mathbb{P}^{2}}, X\right)\right)^{n}$ and $\Omega^{4} X$.

(4) First we define a left action of $\Omega^{4} X$ on $\mathcal{M}_{*} X$. Given a function $f \in \mathcal{M}_{*} X$ we let $s_{f}$ be the supremum of the set of $t \in(-\infty, 0]$ such that $f(s, \mathbf{x})=*$ for any $s \leq t$ and any $\mathbf{x} \in I^{3}$. Then, given $g \in \Omega^{4} X$ we define $g \cdot f \in \mathcal{M}_{*} X$ by:

$$
g \cdot f(t, \mathbf{x})= \begin{cases}f(t, \mathbf{x}), & \text { if } t \geq s_{f} \\ g\left(t-s_{f}, \mathbf{x}\right), & \text { if } t<s_{f}\end{cases}
$$

This action preserves the subspaces $\Omega^{4} X, \operatorname{Map}_{*}\left(\overline{\mathbb{P}^{2}}, X\right)$ and $\operatorname{Map}_{*}\left(\#_{n} \overline{\mathbb{P}^{2}}, X\right)$ and clearly $\left(g_{1} \cdot g_{2}\right) \cdot f=g_{1} \cdot\left(g_{2} \cdot f\right)$. Since $\left(\mathcal{M}_{*} X\right)^{n} \cong \mathcal{M}_{*}\left(X^{n}\right)$, we get an associative product on $\left(\Omega^{4} X\right)^{n}$ and a left action of $\left(\Omega^{4} X\right)^{n}$ on $\left(\mathcal{M}_{*} X\right)^{n}$.

(5) We now define a map $\omega:\left(\mathcal{M}_{*} X\right)^{n} \rightarrow \mathcal{M}_{*} X$ by concatenation in the second variable. Given $\mathbf{f}=\left(f_{1}, \ldots, f_{n}\right) \in\left(\mathcal{M}_{*} X\right)^{n}$, for each $i=1, \ldots, n$ and $x_{1} \in[(i-1) / n, i / n]$, we let $\omega(\mathbf{f})\left(t, x_{1}, x_{2}, x_{3}\right)=f_{i}\left(t, n x_{1}-i+1, x_{2}, x_{3}\right)$. Then, given $\mathbf{f} \in\left(\mathcal{M}_{*} X\right)^{n}$ and $\mathbf{g} \in\left(\Omega^{4} X\right)^{n}$, we have $\omega(\mathbf{g} \cdot \mathbf{f})=\omega(\mathbf{g}) \cdot \omega(\mathbf{f})$. The map $\omega$ restricts to define maps $\left(\Omega^{4} X\right)^{n} \rightarrow \Omega^{4} X$ and $\left(\operatorname{Map}_{*}\left(\overline{\mathbb{P}^{2}}, X\right)\right)^{n} \rightarrow$ $\operatorname{Map}_{*}\left(\#_{n} \overline{\mathbb{P}^{2}}, X\right)$. We define a right action of $\left(\Omega^{4} X\right)^{n}$ on $\Omega^{4} X$ by $f \cdot \mathbf{g}=$ $f \cdot \omega(\mathbf{g})$.

We represent by

$$
\mathrm{B}\left(\Omega^{4} X,\left(\Omega^{4} X\right)^{n},\left(\operatorname{Map}_{*}\left(\overline{\mathbb{P}^{2}}, X\right)\right)^{n}\right)
$$

the bar construction induced by the actions in (4) and (5) (see section 2.1.3).

Theorem 1.5 is a special case of the following theorem, when $X=B S U(\mathbb{V})$ :

Theorem 1.5 ${ }^{*}$. The maps $\Omega^{4} X \times\left(\left(\Omega^{4} X\right)^{n}\right)^{k} \times\left(\operatorname{Map}_{*}\left(\overline{\mathbb{P}^{2}}, X\right)\right)^{n} \rightarrow \operatorname{Map}_{*}\left(\#_{n} \overline{\mathbb{P}^{2}}, X\right)$ sending $\left(g, \mathbf{f}_{1}, \ldots, \mathbf{f}_{k}, \mathbf{f}\right)$ to $g \cdot \omega\left(\mathbf{f}_{1} \cdots \cdot \mathbf{f}_{k} \cdot \mathbf{f}\right)$ induce a map

$$
h: \mathrm{B}\left(\Omega^{4} X,\left(\Omega^{4} X\right)^{n},\left(\operatorname{Map}_{*}\left(\overline{\mathbb{P}^{2}}, X\right)\right)^{n}\right) \rightarrow \operatorname{Map}_{*}\left(\#_{n} \overline{\mathbb{P}^{2}}, X\right)
$$


which is a homotopy equivalence.

Proof. We have a commutative diagram:

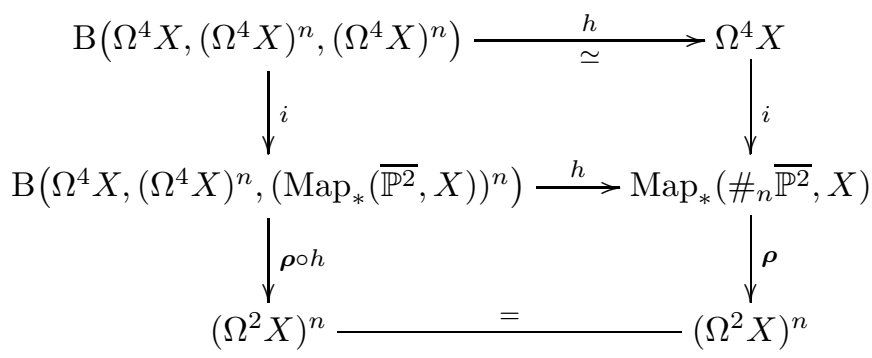

The maps $i$ are inclusions and the map $\rho$ is the one defined above in 9.2(3). The right vertical maps are induced by the cofiber sequence $\bigvee_{n} S^{2} \rightarrow \#_{n} \overline{\mathbb{P}^{2}} \rightarrow S^{4}$, and hence they form a fibration sequence. Since the top row is a homotopy equivalence (see Proposition 2.4), to finish the proof we only have to show that the left vertical maps form a fibration sequence. The map $\boldsymbol{\rho} \circ h$ is induced by the composition

$$
\Omega^{4} X \times\left(\operatorname{Map}_{*}\left(\overline{\mathbb{P}^{2}}, X\right)\right)^{n} \stackrel{p_{2}}{\longrightarrow}\left(\operatorname{Map}_{*}\left(\overline{\mathbb{P}^{2}}, X\right)\right)^{n} \stackrel{\prod \rho}{\longrightarrow}\left(\Omega^{2} X\right)^{n}
$$

where $p_{2}$ is projection onto the second factor and $\rho: \operatorname{Map}_{*}\left(\overline{\mathbb{P}^{2}}, X\right) \rightarrow \Omega^{2} X$ is the map defined above in 9.2(2). The homotopy fiber $F_{\boldsymbol{\rho} \circ h}$ of the map $\boldsymbol{\rho} \circ h$ is the fiber product:

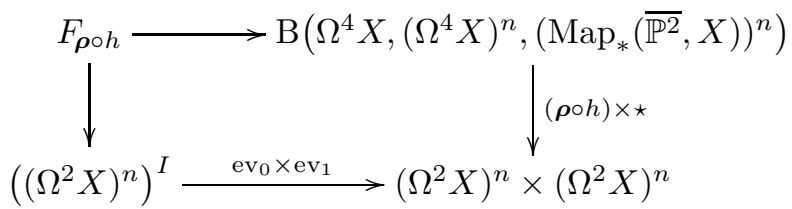

where, given a path $\gamma$ in $\left(\Omega^{2} X\right)^{n}$, $\mathrm{ev}_{0}(\gamma)=\gamma(0)$ and $\mathrm{ev}_{1}(\gamma)=\gamma(1)$, so from 33 , Corollary 11.6] we see that $F_{\rho \circ h}=\mathrm{B}\left(\Omega^{4} X,\left(\Omega^{4} X\right)^{n}, F_{\rho}^{n}\right)$, where $F_{\rho}$ is the homotopy fiber of the map $\rho$. Since the inclusion $\Omega^{4} X \rightarrow F_{\rho}$ is a homotopy equivalence, and the simplicial spaces involved are good, it follows from [44, Proposition A.1] that the map

$$
\mathrm{B}\left(\Omega^{4} X,\left(\Omega^{4} X\right)^{n},\left(\Omega^{4} X\right)^{n}\right) \rightarrow \mathrm{B}\left(\Omega^{4} X,\left(\Omega^{4} X\right)^{n}, F_{\rho}^{n}\right)
$$

is a homotopy equivalence. This concludes the proof.

We now study the rank-stable limit. Consider the $\mathscr{I}$-functors (see [34, page 16]):

$$
X^{\mathbb{V}}=\frac{S U(\mathbb{V} \oplus \mathbb{V})}{S U(\mathbb{V}) \times S U(\mathbb{V})}, \quad P^{\mathbb{V}}=\frac{S U(\mathbb{V} \oplus \mathbb{V})}{1 \times S U(\mathbb{V})}, \quad E^{\mathbb{V}}=P^{\mathbb{V}} \times_{S U(\mathbb{V})} \mathbb{V} .
$$

For each $\mathbb{V}$ the natural map $E^{\mathbb{V}} \rightarrow X^{\mathbb{V}}$ is an $S U(\mathbb{V})$ vector bundle and there is a canonical isomorphism of the fiber $E_{*}^{\mathbb{V}}$ over the basepoint with $\mathbb{V}$. For $\operatorname{dim} \mathbb{V}<\infty$ let $\xi^{\mathbb{V}}$ be the canonical universal connection on $E^{\mathbb{V}}$ [37, section 2]. Then pullback induces a map of $\mathscr{I}_{*}$-functors $\psi_{\mathbb{V}}: \operatorname{Map}\left(\#{ }_{J} \overline{\mathbb{P}^{2}}, X^{\mathbb{V}}\right) \rightarrow \mathscr{C}_{J}^{\mathbb{V}}$ which extends uniquely to a morphism of $\mathscr{I}$-functors by passage to limits.

If $\mathbb{H}$ is a universe (Definition 2.13), then we can identify the classifying space $B S U$ and the universal bundle $E S U$ with $X^{\mathbb{H}}$ and $P^{\mathbb{H}}$ respectively. The maps $\psi_{\mathbb{V}}$ induce a map of $\mathscr{L}^{\mathbb{H}}$-algebras $\operatorname{Map}\left(\# \#_{J} \overline{\mathbb{P}^{2}}, X^{\mathbb{H}}\right) \rightarrow \mathscr{C}_{J, k}^{\mathbb{H}}$ which is a homotopy equivalence (see [2, page 101, second paragraph]). Let $n=\# J$ and consider the homotopy 
coherent bar construction $\mathfrak{B}\left(\Omega^{4} B S U,\left(\Omega^{4} B S U\right)^{n},\left(\operatorname{Map}_{*}\left(\overline{\mathbb{P}^{2}}, B S U\right)\right)^{n}\right)$ constructed as in Definition 5.2. We will now see how this bar construction is related to the one in equation (9.3).

Proposition 9.4. There is a homotopy commutative diagram:

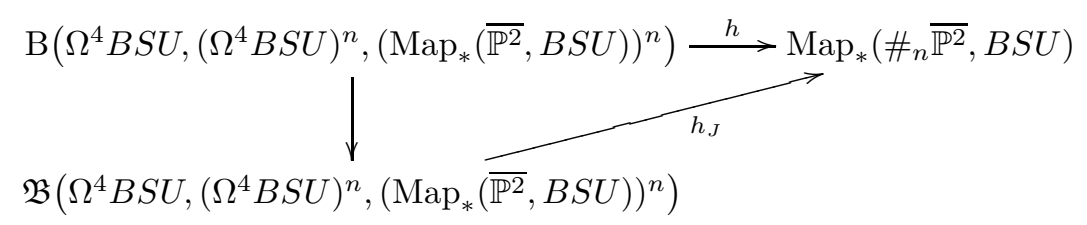

where all the maps are homotopy equivalences.

Proof. The idea of the proof is the same as in the proof of Theorem 8.47. Let $D_{n} X \subset\left(\mathcal{M}_{*} X\right)^{n}$ be the subspace of $n$-tuples $\left(f_{1}, \ldots, f_{n}\right)$ such that, for all $x \in$ $(-\infty, 0] \times I^{3}$ we have $f_{i}(x) \neq *$ for at most one $i$. We then have a map $\mu: D_{n} X \rightarrow$ $\mathcal{M}_{*} X$ with $\mu\left(f_{1}, \ldots, f_{n}\right)(x)=f_{i}(x)$ if $f_{i}(x) \neq *$ for some $i$, and $\mu\left(f_{1}, \ldots, f_{n}\right)(x)=*$ otherwise. An isometry $\alpha: \mathbb{V} \rightarrow \mathbb{V}^{\prime}$ induces a map $X^{\mathbb{V}} \rightarrow X^{\mathbb{V}^{\prime}}$ and hence a map $\mathcal{M}_{*} X^{\mathbb{V}} \rightarrow \mathcal{M}_{*} X^{\mathbb{V}^{\prime}}$. Let $\imath_{i}: \mathbb{V} \rightarrow \mathbb{V}^{n}$ be inclusion into the $i$-th factor. Then the following diagrams are commutative (compare with Proposition 7.46 ):
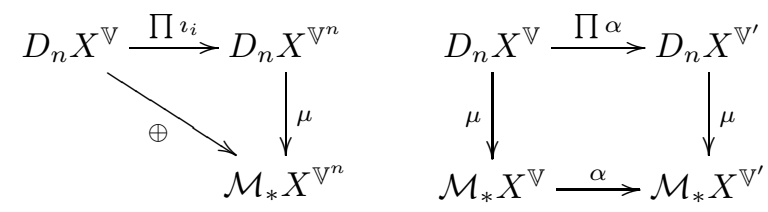

where $\prod \imath_{i}$ and $\prod \alpha$ denote the maps obtained by restricting the domain and the codomain of the product maps. The analogue of the maps in equation (8.48) are defined as follows: Let $\mathcal{P}$ be as in the proof of Theorem 8.47. let $\imath_{i}: \mathbb{H} \rightarrow \mathbb{H}^{n}$ be inclusion into the $i$-th factor and for each $f \in \mathcal{P}(n)$ define a map $\mu_{f}$ as the composition

$$
\mu_{f}: D_{n} X^{\mathbb{H}} \stackrel{\prod\left(f \circ \imath_{i}\right)}{\longrightarrow} D_{n} X^{\mathbb{H}} \stackrel{\mu}{\longrightarrow} \mathcal{M}_{*} X^{\mathbb{H}}
$$

We now express the maps of Definition 9.2(4)(5) in terms of $\mu$ as follows:

(1) Let $j_{i}: \mathcal{M}_{*} X \rightarrow\left(\mathcal{M}_{*} X\right)^{n}$ be inclusion into the $i$-th factor and consider the composition $\omega_{i}=\omega \circ j_{i}$. For each $i$, the map $\omega_{i}$ is an embedding and the map $\omega$ is the composition

$$
\omega:\left(\mathcal{M}_{*} X\right)^{n} \stackrel{\prod \omega_{i}}{\longrightarrow} D_{n} X \stackrel{\mu}{\rightarrow} \mathcal{M}_{*} X .
$$

(2) For each $s \leq 0$ let $\tau_{s}: \Omega^{4} X \rightarrow \Omega^{4} X$ be defined by

$$
\tau_{s} g(t, \mathbf{x})= \begin{cases}g(t-s, \mathbf{x}) & t<s \\ * & t \geq s\end{cases}
$$

For each $g \in \mathcal{M}_{*} X$ let $s_{g}$ be as in Definition $9.2(4)$. Given spaces $X_{1}, \ldots, X_{m+1}$ and $\left(g_{1}, \ldots, g_{m}, g_{m+1}\right) \in\left(\prod_{i=1}^{m} \Omega^{4} X_{i}\right) \times \mathcal{M}_{*} X_{m+1}$, for each $i$ let $r_{i}=$ $s_{g_{i+1}}+\cdots+s_{g_{m+1}}$ and let $\tau:\left(\prod \Omega^{4} X_{i}\right) \times \mathcal{M}_{*} X_{m+1} \rightarrow\left(\prod \Omega^{4} X_{i}\right) \times \mathcal{M}_{*} X_{m+1}$ be defined by

$$
\tau\left(g_{1}, \ldots, g_{m+1}\right)=\left(\tau_{r_{1}} g_{1}, \ldots, \tau_{r_{m}} g_{m}, g_{m+1}\right) .
$$


If $X_{1}=\cdots=X_{m+1}=X$ then $\mu \circ \tau\left(g_{1}, \ldots, g_{m+1}\right)=g_{1} \cdot \ldots \cdot g_{m} \cdot g_{m+1}$. Note that the map $\tau$ is an embedding with image the subspace of $m+1$-tuples $\left(g_{1}, \ldots, g_{m+1}\right) \in \prod_{i} \mathcal{M}_{*} X_{i}$ such that $\operatorname{supp} g_{i} \subset\left(-\infty, s_{g_{i+1}}\right]$.

Let $\mathcal{P}^{J}$ and $\mathcal{P}_{J}$ be as in the proof of Theorem 8.47 and consider the functor $\mathcal{F}_{J}$ : $\Delta\left(\mathcal{P}_{J}, \mathcal{P}^{J}\right)^{\text {op }} \rightarrow$ Top with $\mathcal{F}_{J}(m)$ equal to the image of the following embedding $($ where $n=\# J)$ :

$$
\begin{aligned}
& \Omega^{4} B S U \times\left(\left(\Omega^{4} B S U\right)^{n}\right)^{m} \times\left(\operatorname{Map}\left(\overline{\mathbb{P}^{2}}, B S U\right)\right)^{n} \\
& \stackrel{\mathbf{1} \times\left(\prod \omega_{i}\right)^{m} \times \prod \omega_{i}}{\longrightarrow} \Omega^{4} B S U \times\left(\left(\Omega^{4} B S U\right)^{n}\right)^{m} \times\left(\operatorname{Map}\left(\overline{\mathbb{P}^{2}}, B S U\right)\right)^{n} \\
&=\Omega^{4} B S U \times\left(\Omega^{4}\left(B S U^{n}\right)\right)^{m} \times \operatorname{Map}\left(\overline{\mathbb{P}^{2}}, B S U^{n}\right) \\
& \stackrel{\tau}{\rightarrow} \Omega^{4} B S U \times\left(\Omega^{4}\left(B S U^{n}\right)\right)^{m} \times \operatorname{Map}\left(\overline{\mathbb{P}^{2}}, B S U^{n}\right)
\end{aligned}
$$

and defined on morphisms by using the maps $\mu_{f}$. The proof now proceeds exactly as in the proof of Theorem 8.47

\section{Homology}

In this section we prove Theorem 1.6. We'll always work with a fixed countably infinite dimensional vector space $\mathbb{H}$ which we omit from the notation. We begin by computing the homology of $\left\|\mathfrak{B}_{I}\right\|$. Fix a point $x \in \mathbb{C}^{2}$ and consider the homology rings $R=H_{*}\left(\mathfrak{M}_{\emptyset}\right)$ and $M=H_{*}\left(\mathfrak{M}_{x}\right)$. It was shown in [28, Theorem 1.2], 40, Theorem 3], [8, Theorem 1.1] that $\mathfrak{M}_{\emptyset} \simeq \coprod_{k \geq 0} B U(k)$ and $\mathfrak{M}_{x} \simeq \coprod_{k \geq 0} B U(k) \times$ $B U(k)$ as $E_{\infty}$-spaces; pullback $\pi^{*}: \mathfrak{M}_{\emptyset} \rightarrow \mathfrak{M}_{x}$ is induced by the diagonal inclusions $\Delta_{k}: B U(k) \rightarrow B U(k) \times B U(k)$ (see [9, equation (2)]). Thus we have $R=\bigoplus R_{k}$ and $M=\bigoplus M_{k}$ where $R_{k} \cong H_{*}(B U(k))$ and $M_{k} \cong H_{*}(B U(k) \times B U(k)) \cong R_{k} \otimes R_{k}$. If we write

$$
R=\mathbb{Z}\left[r_{i} ; i=0,1,2, \ldots\right]
$$

where $r_{i} \in H_{2 i}(B U(1))$ is dual to $c_{1}^{i}$ (see [35, Theorem 21.4.3]), then $R_{k}$ is the $\mathbb{Z}$-submodule of homogeneous polynomials of degree $k$. The inclusion $\amalg(B U(k) \times$ $B U(k)) \rightarrow(\amalg B U(k)) \times(\amalg B U(k))$ induces an inclusion

$$
M=\bigoplus_{k} R_{k} \otimes R_{k} \subset R \otimes R=\mathbb{Z}\left[x_{i}, y_{j} ; i, j \geq 0\right]
$$

(where $x_{i}$ and $y_{i}$ have homological degree $2 i$ ); namely, $M$ is the subring generated by $x_{i} y_{j}$, with $i, j \geq 0$. Consider the bigrading on the $\mathbb{Z}$-modules $R$ and $M$, where the bidegree $(i, k)$ summand consist of the elements in $R_{k}, M_{k}$ with homological degree $2 i$; in particular:

$$
\operatorname{deg} r_{i}=(i, 1) \quad \text { and } \quad \operatorname{deg} x_{i} y_{j}=(i+j, 1) .
$$

The diagonal inclusions $\Delta_{k}: B U(k) \rightarrow B U(k) \times B U(k)$ induce a homomorphism $\Delta_{*}: R \rightarrow M$ of bigraded rings with $\Delta_{*}\left(r_{k}\right)=\sum_{i+j=k} x_{i} y_{j}$, which makes $M$ into an $R$-module.

Proposition 10.2. Let $T_{k} \subset \mathbb{Z}\left[x_{i} ; i \geq k\right]$ be the $\mathbb{Z}$-submodule of homogeneous polynomials of degree $k$, let $N_{k}=T_{k} \otimes R_{k} \subset M_{k}$ and let $N=\bigoplus_{k} N_{k} \subset M$. Then $M$ is a free module over $R$ with basis $N$.

Proof. Let $\phi: R \otimes N \rightarrow M$ be the homomorphism of bigraded $\mathbb{Z}$-modules induced by $\Delta_{*}$. We want to show that $\phi$ is an isomorphism. We begin by showing that $\phi$ is 
surjective. First we need to establish some notation. Let $A, B$ be eventually zero sequences of non-negative integers:

$$
A=\left(a_{0}, a_{1}, \ldots, a_{n}, \ldots\right), \quad B=\left(b_{0}, b_{1} \ldots, b_{n}, \ldots\right) .
$$

We order these sequences by lexicographic order. Let

$$
x^{A} y^{B}=x_{0}^{a_{0}} x_{1}^{a_{1}} \ldots x_{n}^{a_{n}} \ldots y_{0}^{b_{0}} \ldots y_{n}^{b_{n}} \ldots \in R \otimes R,
$$

and let $|A|=\sum_{k} a_{k}$ and $|B|=\sum_{k} b_{k}$. Then $x^{A} y^{B} \in M$ if and only if $|A|=|B|$, and these monomials form a $\mathbb{Z}$-basis for $M$. We define a total order on the set of monomials $x^{A} y^{B}$ as follows:

$$
\begin{aligned}
x^{A_{1}} y^{B_{1}}>x^{A_{2}} y^{B_{2}} \text { if and only if } & \left|A_{1}\right|>\left|A_{2}\right|, \text { or } \\
& \left|A_{1}\right|=\left|A_{2}\right| \text { and } A_{1}>A_{2}, \text { or } \\
& A_{1}=A_{2} \text { and } B_{1}<B_{2}
\end{aligned}
$$

(notice the reversed order on the $B$ 's). We will show by induction on the ordering of the monomials that any monomial $x^{A} y^{B} \in M$ is in the image of $\phi$. The statement is clearly true for $1 \in M$ and whenever $x^{A} y^{B} \in N_{n}$ for some $n$, so fix a monomial $x^{A_{1}} y^{B_{1}} \in M_{n} \backslash N_{n}$ and assume, by induction hypothesis, that every monomial $x^{A_{2}} y^{B_{2}}$ strictly smaller than $x^{A_{1}} y^{B_{1}}$ is in the image of $\phi$. Since $x^{A_{1}} y^{B_{1}} \notin N_{n}$, there is a $k \leq n$ and a sequence $A=\left(a_{0}, a_{1}, \ldots\right)$ with $|A|=n-1$ and $a_{i}=0$ for $i<k-1$ such that $x^{A_{1}}=x_{k-1} x^{A}$. Also, since $k \leq n$, we can write $y^{B_{1}}=$ $y_{n_{1}} y_{n_{2}-1} \ldots y_{n_{k}-k+1} y^{B}$ with $n_{1}<n_{2}<\cdots<n_{k}$ and $B=\left(b_{0}, b_{1}, \ldots\right)$ a sequence with $|B|=n-k$ and $b_{i}=0$ for $i<n_{k}-k+1$. Set $x_{q}=y_{q}=0$ for $q<0$ and consider the following element in $\mathbb{Z}\left[x_{i}, y_{j}\right]$ :

$$
d=x^{A} y^{B} \operatorname{det}\left(\begin{array}{ccc}
y_{n_{1}} & \ldots & y_{n_{k}} \\
y_{n_{1}-1} & \ldots & y_{n_{k}-1} \\
\vdots & \ddots & \vdots \\
y_{n_{1}-k+2} & \cdots & y_{n_{k}-k+2} \\
r_{n_{1}} & \cdots & r_{n_{k}}
\end{array}\right)
$$

(where, abusing notation, we write $r_{i}$ instead of $\Delta_{*} r_{i}$ ). Using Laplace's formula in the last row we find that $d=\sum_{i=0}^{k} r_{n_{i}} p_{i}$ with $p_{i} \in M_{n-1}$. In particular, all monomials in each polynomial $p_{i}$ are strictly smaller than $x^{A_{1}} y^{B_{1}}$ so, by the induction hypothesis, we have $d \in \operatorname{Im} \phi$. Now using the identities $r_{n_{i}}=\sum x_{\ell} y_{n_{i}-\ell}$ in the last row we find that:

$$
d=\sum_{\ell=0}^{\infty} x_{\ell} x^{A} y^{B} \operatorname{det}\left(\begin{array}{ccc}
y_{n_{1}} & \ldots & y_{n_{k}} \\
y_{n_{1}-1} & \ldots & y_{n_{k}-1} \\
\vdots & \ddots & \vdots \\
y_{n_{1}-k+2} & \ldots & y_{n_{k}-k+2} \\
y_{n_{1}-\ell} & \ldots & y_{n_{k}-\ell}
\end{array}\right) .
$$

Notice that the terms in the sum vanish for $\ell=0, \ldots, k-2$ and $x_{\ell} x^{A}<x_{k-1} x^{A}$ for $\ell \geq k$. Also, for $\ell=k-1$, the leading term of the determinant is the product of the entries in the main diagonal. Thus we can write

$$
d=x_{k-1} x^{A} y^{B} y_{n_{1}} y_{n_{2}-1} \ldots y_{n_{k}-k+1}+p=x^{A_{1}} y^{B_{1}}+p \in \operatorname{Im} \phi
$$

where $p$ is a finite sum of monomials strictly smaller than $x^{A_{1}} y^{B_{1}}$. Using the induction hypothesis we conclude that $x^{A_{1}} y^{B_{1}} \in \operatorname{Im} \phi$, which finishes the proof of 
the surjectivity of $\phi$. Since $R \otimes N$ and $M$ are free $\mathbb{Z}$-modules, finitely generated in each bidegree, in order to show that $\phi$ is an isomorphism we only need to check that the dimensions over $\mathbb{Z}$ match, which we will prove in Lemma 10.5 below.

Before we continue we introduce some notation. Let $(t ; q)_{n}=\prod_{i=0}^{n-1}\left(1-t q^{i}\right)$ denote the $q$-Pochhammer symbol and let $(q)_{n}=(q ; q)_{n}$. Also consider the $q$ multinomial

$$
\left(\begin{array}{c}
k \\
i_{1}, \ldots, i_{n}
\end{array}\right)_{q}=\frac{(q)_{k}}{\prod_{j=1}^{n}(q)_{i_{j}}} \quad\left(k=i_{1}+\cdots+i_{n}\right)
$$

and let $\left(\begin{array}{c}k \\ i\end{array}\right)_{q}=\left(\begin{array}{c}k \\ i, k-i\end{array}\right)_{q}$ be the $q$-binomial. One easily checks that

$$
\left(\begin{array}{c}
k \\
i
\end{array}\right)_{q}=q^{i}\left(\begin{array}{c}
k-1 \\
i
\end{array}\right)_{q}+\left(\begin{array}{c}
k-1 \\
i-1
\end{array}\right)_{q} .
$$

Lemma 10.4. For any $n, j \in \mathbb{Z}$ with $n \geq j \geq 0$ we have

$$
A_{n, j}=\sum_{k=0}^{n-j} \frac{(q)_{n}}{(q)_{k+j}}\left(\begin{array}{c}
n-j \\
k
\end{array}\right)_{q} q^{k^{2}+k j}=1 .
$$

Proof. The proof is by induction on $n-j$. The result is clear for $n=j$. Using the relations $\left(\begin{array}{l}n \\ k\end{array}\right)_{q}=\left(\begin{array}{c}n-1 \\ k\end{array}\right)_{q}+q^{n-k}\left(\begin{array}{c}n-1 \\ k-1\end{array}\right)_{q}$ and $(q)_{n}=\left(1-q^{n}\right)(q)_{n-1}$, we get:

$$
\begin{aligned}
A_{n, j} & =\sum_{k=0}^{n-j-1} \frac{(q)_{n}}{(q)_{k+j}}\left(\begin{array}{c}
n-j-1 \\
k
\end{array}\right)_{q} q^{k^{2}+k j}+\sum_{k=1}^{n-j} \frac{(q)_{n}}{(q)_{k+j}} q^{n-j-k}\left(\begin{array}{c}
n-j-1 \\
k-1
\end{array}\right)_{q} q^{k^{2}+k j} \\
& =\left(1-q^{n}\right) A_{n-1, j}+\sum_{k=0}^{n-j-1} \frac{(q)_{n}}{(q)_{k+j+1}}\left(\begin{array}{c}
n-j-1 \\
k
\end{array}\right)_{q} q^{n-j-k-1} q^{(k+1)^{2}+(k+1) j} \\
& =\left(1-q^{n}\right) A_{n-1, j}+q^{n} A_{n, j+1}
\end{aligned}
$$

hence $A_{n, j}=1$ by the induction hypothesis.

Consider the bigrading in the $\mathbb{Z}$-modules $R, M$ and $N$ (equation (10.1)). Write the Hilbert series as $P(q, t)=\sum d_{i k} q^{i} t^{k}$ where $d_{i k}$ is the rank of the bidegree $(i, k)$ summand.

Lemma 10.5. The Hilbert series of $R, M$ and $N$ are respectively:

$$
P_{R}(q, t)=\sum_{n=0}^{\infty} \frac{t^{n}}{(q)_{n}} ; \quad P_{M}(q, t)=\sum_{n=0}^{\infty} \frac{t^{n}}{\left((q)_{n}\right)^{2}} ; \quad P_{N}(q, t)=\sum_{n=0}^{\infty} \frac{q^{n^{2}} t^{n}}{\left((q)_{n}\right)^{2}},
$$

and we have $P_{M}(q, t)=P_{R}(q, t) P_{N}(q, t)$.

Remark 10.6. Lemma 10.5 shows that the Hilbert series of $R \otimes N$ equals $P_{R \otimes N}=$ $P_{R} P_{N}=P_{M}$ which concludes the proof of Proposition 10.2.

Proof. Since $R_{k} \cong H^{*}(B U(k))=\mathbb{Z}\left[c_{1}, \ldots, c_{k}\right]$ with $\operatorname{deg} c_{i}=(i, 1)$, and $M_{k}=R_{k} \otimes$ $R_{k}$, the Hilbert series of $R_{k}$ and $M_{k}$ are respectively $1 /(q)_{n}$ and $1 /(q)_{n}^{2}$ from which the formulas for $P_{R}$ and $P_{M}$ immediately follow. The Hilbert series of $\mathbb{Z}\left[x_{i} ; i \geq\right.$ $k] \cong \mathbb{Z}\left[x_{k}\right] \otimes \mathbb{Z}\left[x_{k+1}\right] \otimes \cdots$, where $x_{i}$ has bidegree $(i, 1)$, is

$$
\prod_{i=k}^{\infty} \frac{1}{1-t q^{i}}=\frac{1}{\left(t q^{k} ; q\right)_{\infty}}=\sum_{n=0}^{\infty} \frac{\left(t q^{k}\right)^{n}}{(q)_{n}}
$$


(the second equality is a special case of the $q$-binomial theorem: see [4, Corollary 2.3]). The Hilbert series of $T_{k} \subset \mathbb{Z}\left[x_{i} ; i \geq k\right]$ is obtained by taking the coefficient of $t^{k}$ and hence it is given by $q^{k^{2}} /(q)_{k}$, so the Hilbert series of $N_{k}=T_{k} \otimes R_{k}$ is $q^{k^{2}} /(q)_{k}^{2}$, from which we get the formula for $P_{N}(q, t)$. Writing $P_{M}, P_{R}$ and $P_{N}$ as power series in $t$ and multiplying the series we see that the identity $P_{M}=P_{R} P_{N}$ is equivalent to the identity

$$
\sum_{k=0}^{n} \frac{q^{k^{2}}}{\left((q)_{k}\right)^{2}(q)_{n-k}}=\frac{1}{\left((q)_{n}\right)^{2}}
$$

which follows immediately from Lemma 10.4 by taking $j=0$.

Proposition 10.7. Given a finite set $I \subset \mathbb{C}^{2}$ with cardinality $\# I=n$, we have

$$
H_{*}\left(\left\|\mathfrak{B}_{I}\right\|\right) \cong \underbrace{M \otimes_{R} \cdots \otimes_{R} M}_{n} \cong N^{\otimes n} \otimes R .
$$

Proof. Consider the equivalence of categories $F: \Delta_{I} \rightarrow \Delta$ and let $F_{h *} \mathfrak{B}_{I}: \Delta^{\mathrm{op}} \rightarrow$ Top be Segal's homotopy pushdown construction (see [23, section 5]). Then we have homotopy equivalences $\left\|\mathfrak{B}_{I}\right\| \simeq \operatorname{hcolim}_{\Delta \text { op }} F_{h *} \mathfrak{B}_{I}$ [23. Proposition 5.5] and hcolim $_{\Delta^{\text {op }}} F_{h *} \mathfrak{B}_{I} \simeq\left|\tau F_{h *} \mathfrak{B}_{I}\right|$ (Proposition 2.7(1)). The homology version of Segal's spectral sequence [43, Proposition 5.1], converging to the homology of $\left|\tau F_{h *} \mathfrak{B}_{I}\right|$, has as $E^{1}$ term the simplicial chain complex $E_{\bullet, q}^{1}=H_{q}\left(\tau F_{h *} \mathfrak{B}_{I}\right) \cong H_{q}\left(F_{h *} \mathfrak{B}_{I}\right)$. If $1: \Delta_{I} \rightarrow \Delta_{I}$ denotes the identity functor, then we have weak equivalences $F^{*} F_{h *} \mathfrak{B}_{I} \leftarrow 1^{*} 1_{h *} \mathfrak{B}_{I} \rightarrow \mathfrak{B}_{I}\left[23\right.$, Proposition 5.3] so the functors $F_{h *} \mathfrak{B}_{I}$ and $\mathfrak{B}_{I}$ are naturally equivalent as functors $\Delta^{\mathrm{op}} \rightarrow$ hTop. It follows that the chain complex $H_{q}\left(F_{h * \mathfrak{B}_{I}}\right)$ is isomorphic to the bar complex $\mathrm{B}\left(R, R^{\otimes n}, M^{\otimes n}\right)$. By Proposition 10.2. $M^{\otimes n}$ is a free module over $R^{\otimes n}$ with basis $N^{\otimes n}$ so the spectral sequence collapses and we find that

$$
H_{*}\left(\left\|\mathfrak{B}_{I}\right\|\right) \cong M^{\otimes n} \otimes_{R^{\otimes n}} R \cong N^{\otimes n} \otimes R \cong M \otimes_{R} \cdots \otimes_{R} M
$$

which concludes the proof.

Proposition 10.8. The degree $c_{2}=k$ components $\left\|\mathfrak{B}_{I, k}\right\|$ of the space $\left\|\mathfrak{B}_{I}\right\|$ are simply connected.

Proof. The proof is by induction on $\# I$. For $\# I=0,1$ it immediately follows from Proposition 5.4. For the induction step we use Proposition 5.12. Write $I=J \cup L$ with $\# J, \# L<\# I$ and $J \cap L=\emptyset$, and let

$$
\mathfrak{B}_{J, L, k}(\bullet)=\mathfrak{B}_{\bullet}\left(\left\|F_{i}^{*} \mathfrak{B}_{J}\right\|, \mathfrak{M}_{\emptyset},\left\|F_{i}^{*} \mathfrak{B}_{L}\right\|\right)_{k}, \quad\left\|\mathfrak{B}_{J, L, k}\right\|=\mathfrak{B}\left(\left\|F_{i}^{*} \mathfrak{B}_{J}\right\|, \mathfrak{M}_{\emptyset},\left\|F_{i}^{*} \mathfrak{B}_{L}\right\|\right)_{k} .
$$

Then $\left\|\mathfrak{B}_{I, k}\right\| \simeq\left\|\mathfrak{B}_{J, L, k}\right\|$. By induction hypothesis, the connected components of $\mathfrak{B}_{J, L, k}(m)$ :

$$
\left\|F_{i}^{*} \mathfrak{B}_{J, \alpha_{0}}\right\| \times\left(\prod_{j=1}^{m} \mathfrak{M}_{\emptyset, \alpha_{j}-\alpha_{j-1}}\right) \times\left\|F_{i}^{*} \mathfrak{B}_{L, k-\alpha_{m}}\right\| \quad\left(0 \leq \alpha_{0} \leq \cdots \leq \alpha_{m} \leq k\right)
$$

are simply connected. Let $F_{h *}$ be Segal's homotopy pushdown along the equivalence of categories $F: \Delta\left(\mathscr{L}_{+}, \mathscr{L}\right) \rightarrow \Delta$. The simplicial space obtained by replacing each connected component in $F_{h *} \mathfrak{B}_{J, L, k}$ by a singleton is $\mathrm{B} \bullet\left(*, \mathfrak{C}_{k}, *\right)$, where $\mathfrak{C}_{k}$ is the category $\{0 \rightarrow 1 \rightarrow \cdots \rightarrow k\}$. A choice of basepoint in the connected component of $F_{h *} \mathfrak{B}_{J, L, k}(k)$ corresponding to the composition of arrows $0 \rightarrow 1 \rightarrow \cdots \rightarrow k \in \mathrm{B}_{k}\left(*, \mathfrak{C}_{k}, *\right)$ determines via the face and degeneracy maps, for any $m$, a collection $K_{0}(m) \subset F_{h *} \mathfrak{B}_{J, L, k}(m)$ of basepoints, one in each 
connected component, inducing a simplicial map B. $\left(*, \mathfrak{C}_{k}, *\right) \rightarrow F_{h *} \mathfrak{B}_{J, L, k}$. Let $\tilde{\pi}_{1}\left(F_{h *} \mathfrak{B}_{J, L, k}(m)\right)$ denote the fundamental groupoid with $K_{0}(m)$ as the set of objects and with morphisms the equivalence classes os paths between basepoints. The diagram of spaces $F_{h *} \mathfrak{B}_{J, L, k}$ induces a diagram of groupoids $\tilde{\pi}_{1}\left(F_{h *} \mathfrak{B}_{J, L, k}\right)$ and since the connected components of $F_{h *} \mathfrak{B}_{J, L, k}$ are simply connected, we have an isomorphism of diagrams

$$
\tilde{\pi}_{1}\left(F_{h *} \mathfrak{B}_{J, L, k}\right) \cong \tilde{\pi}_{1}\left(\mathrm{~B}_{\bullet}\left(*, \mathfrak{C}_{k}, *\right)\right) .
$$

By [17, Theorem 1.1] we have natural equivalences of groupoids

$$
\left.\tilde{\pi}_{1} \underset{\Delta^{\mathrm{op}}}{\operatorname{hcolim}} F_{h *} \mathfrak{B}_{J, L, k}\right) \cong \underset{\Delta^{\mathrm{op}}}{\operatorname{hcolim}} \tilde{\pi}_{1}\left(F_{h *} \mathfrak{B}_{J, L, k}\right)
$$

and

$$
\left.\tilde{\pi}_{1} \underset{\Delta^{\text {op }}}{\operatorname{hcolim}} \mathrm{B}_{\bullet}\left(*, \mathfrak{C}_{k}, *\right)\right) \cong \underset{\Delta^{\mathrm{op}}}{\operatorname{hcolim}} \tilde{\pi}_{1}\left(\mathrm{~B} \bullet\left(*, \mathfrak{C}_{k}, *\right)\right) .
$$

Since hcolim $\left.\Delta^{\mathrm{op}} \mathrm{B}_{\bullet}\left(*, \mathfrak{C}_{k}, *\right)\right) \simeq \mathrm{B}\left(*, \mathfrak{C}_{k}, *\right)$ is contractible,

$$
\begin{aligned}
\tilde{\pi}_{1}\left(\left\|\mathfrak{B}_{J, L, k}\right\|\right) & \cong \tilde{\pi}_{1}\left(\underset{\Delta^{\mathrm{op}}}{\operatorname{hcolim}} F_{h *} \mathfrak{B}_{J, L, k}\right) \cong \underset{\Delta^{\mathrm{op}}}{\operatorname{hcolim}} \tilde{\pi}_{1}\left(F_{h *} \mathfrak{B}_{J, L, k}\right) \\
& \left.\cong \underset{\Delta^{\mathrm{op}}}{\operatorname{hcolim}} \tilde{\pi}_{1}\left(\mathrm{~B} \cdot\left(*, \mathfrak{C}_{k}, *\right)\right) \cong \underset{\Delta^{\mathrm{op}}}{\tilde{\pi}_{1}\left(\operatorname{hcolim}_{\bullet}\right.} \mathrm{B}_{\bullet}\left(* \mathfrak{C}_{k}, *\right)\right) \cong\{1\}
\end{aligned}
$$

which finishes the proof.

As in Lemma 10.5, we write the Hilbert series of $H_{*}\left(\left\|\mathfrak{B}_{I}\right\|\right)$ as $P_{I}(q, t)=$ $\sum d_{i k} q^{i} t^{k}$ where $d_{i k}$ is the rank of the bidegree $(i, k)$ summand. Also, given power series $s_{1}, s_{2} \in \mathbb{Z}[[q]]$ we write $s_{1}=s_{2}+\mathcal{O}\left(q^{m}\right)$ if $s_{1}=s_{2}$ in $\mathbb{Z}[[q]] /\left\langle q^{m}\right\rangle$.

Lemma 10.9. Let $I \subset \mathbb{C}^{2}$ be a finite set with cardinality $n$. Then the Hilbert series of $H_{*}\left(\left\|\mathfrak{B}_{I}\right\|\right)$ is given by the q-series:

$$
P_{I}(q, t)=\sum_{k=0}^{\infty} \frac{p_{n, k}(q)}{(q)_{k}^{2}} t^{k}, \quad \text { where } \quad p_{n, k}=\sum_{i_{1}+\cdots+i_{n}=k} q^{i_{2}^{2}+\cdots+i_{n}^{2}}\left(\begin{array}{c}
k \\
i_{1}, \ldots, i_{n}
\end{array}\right)_{q}^{2} .
$$

The polynomials $p_{n, k}$ satisfy:

(1) $p_{1, k}=1, p_{2, k}=\left(\begin{array}{c}2 k \\ k\end{array}\right)_{q}, p_{n, 1}=1+(n-1) q$ and

$$
p_{n, 2}=1+(n-1) q+\frac{1}{2}(n-1)(n+2) q^{2}+(n-1)^{2} q^{3}+\frac{1}{2} n(n-1) q^{4} ;
$$

(2) For any $n, k$ we have $(q)_{\infty}^{n-1} p_{n, k}=1+\mathcal{O}\left(q^{k+1}\right)$;

(3) For $2 \leq n \leq k+1$ we have

$$
(q)_{\infty}^{n-1} p_{n, k}=1-\left(2^{n}-2\right) \sum_{i=k+1}^{2 k-n+2} q^{i}-\left(2^{n}-3\right) q^{2 k-n+3}+\mathcal{O}\left(q^{2 k-n+4}\right) .
$$

Proof. By Proposition 10.7, the homology of $\left\|\mathfrak{B}_{I}\right\|$ is isomorphic to the tensor product $M \otimes N^{\otimes(n-1)}$ so its Hilbert series is given by

$$
P_{I}(q, t)=\left(\sum_{k=0}^{\infty} \frac{t^{k}}{(q)_{k}^{2}}\right)\left(\sum_{k=0}^{\infty} \frac{q^{k^{2}} t^{k}}{(q)_{k}^{2}}\right)^{n-1}=\sum_{k=0}^{\infty} \frac{p_{n, k}}{(q)_{k}^{2}} t^{k}
$$

with $p_{n, k}$ as in equation (10.10). The cases $k=1,2$ and $n=1$ are easily computed, while the case $n=2$ is a special case of the $q$-Vandermonde identities [45, Solution to exercise 1.100 , page 188$]$. 
We prove statement (2) by induction on $n$. Statement (2) holds for $n=1,2$ since, by (1), we have $(q)_{\infty} p_{2, k}=\left(q^{k+1}\right)_{\infty}\left(q^{k+1}\right)_{k}$. Assume (2) holds for $n-1$. The polynomials $p_{n, k}$ satisfy the recurrence relation:

$$
p_{n, k}=\sum_{i=0}^{k} q^{i^{2}}\left(\begin{array}{l}
k \\
i
\end{array}\right)_{q}^{2} p_{n-1, k-i}
$$

so we find that

$$
(q)_{\infty}^{n-2} p_{n, k}=\sum_{a=0}^{k} q^{a^{2}}\left(\begin{array}{l}
k \\
a
\end{array}\right)_{q}^{2}(q)_{\infty}^{n-2} p_{n-1, k-a}=\sum_{a=0}^{k} q^{a^{2}}\left(\begin{array}{l}
k \\
a
\end{array}\right)_{q}^{2}\left(1+\mathcal{O}\left(q^{k-a+1}\right)\right) .
$$

Since $q^{a^{2}} q^{k-a+1}=\mathcal{O}\left(q^{k+1}\right)$, we get:

$$
(q)_{\infty}^{n-1} p_{n, k}=(q)_{\infty} \sum_{a=0}^{k} q^{a^{2}}\left(\begin{array}{l}
k \\
a
\end{array}\right)_{q}^{2}+\mathcal{O}\left(q^{k+1}\right)=(q)_{\infty} p_{2, k}+\mathcal{O}\left(q^{k+1}\right)=1+\mathcal{O}\left(q^{k+1}\right)
$$

which finishes the proof of (2).

We now prove (3) by induction on $n$. For $n=2$ we have $(q)_{\infty} p_{2, k}=\left(q^{k+1}\right)_{\infty}\left(q^{k+1}\right)_{k}$ and the result follows. Assume (3) holds for $n-1$. Then

$$
\begin{aligned}
(q)_{\infty}^{n-2} p_{n, k} & =\sum_{a=0}^{k} q^{a^{2}}\left(\begin{array}{l}
k \\
a
\end{array}\right)_{q}^{2}(q)_{\infty}^{n-2} p_{n-1, k-a} \\
& =\sum_{a=0}^{k} q^{a^{2}}\left(\begin{array}{l}
k \\
a
\end{array}\right)_{q}^{2}\left(1-\left(2^{n-1}-2\right) \sum_{i=k-a+1}^{2 k-2 a-n+3} q^{i}+\mathcal{O}\left(q^{2 k-2 a-n+4}\right)\right) .
\end{aligned}
$$

For $a \neq 1$ we have $a^{2}+2 k-2 a-n+4=(a-1)^{2}+2 k-n+3 \geq 2 k-n+4$, so:

$$
q^{a^{2}} \sum_{i=k-a+1}^{2 k-2 a-n+3} q^{i}=q^{a^{2}-a} \sum_{i=k+1}^{2 k-a-n+3} q^{i}=q^{a^{2}-a} \sum_{i=k+1}^{2 k-n+3} q^{i}+\mathcal{O}\left(q^{2 k-n+4}\right)
$$

while for $a=1$ we have, by the induction hypothesis,

$$
q(q)_{\infty}^{n-2} p_{n-1, k-1}=q-\left(2^{n-1}-2\right) \sum_{i=k+1}^{2 k-n+3} q^{i}+q^{2 k-n+3}+\mathcal{O}\left(q^{2 k-n+4}\right) .
$$

Thus we have:

$$
\begin{aligned}
(q)_{\infty}^{n-2} p_{n, k}=\sum_{a=0}^{k} q^{a^{2}}\left(\begin{array}{l}
k \\
a
\end{array}\right)_{q}^{2}-\left(2^{n-1}-2\right) \sum_{i=k+1}^{2 k-n+3} q^{i} \sum_{a=0}^{k} q^{a^{2}-a}\left(\begin{array}{l}
k \\
a
\end{array}\right)_{q}^{2} & \\
& +q^{2 k-n+3}+\mathcal{O}\left(q^{2 k-n+4}\right) .
\end{aligned}
$$

Now, using equation (10.3) and the $q$-Vandermonde identities we get:

$$
\sum_{a=0}^{k} q^{a^{2}-a}\left(\begin{array}{l}
k \\
a
\end{array}\right)_{q}^{2}=\sum_{a=0}^{k-1} q^{a^{2}}\left(\begin{array}{l}
k \\
a
\end{array}\right)_{q}\left(\begin{array}{c}
k-1 \\
a
\end{array}\right)_{q}+\sum_{a=1}^{k} q^{a^{2}-a}\left(\begin{array}{l}
k \\
a
\end{array}\right)_{q}\left(\begin{array}{c}
k-1 \\
a-1
\end{array}\right)_{q}=2\left(\begin{array}{c}
2 k-1 \\
k
\end{array}\right)_{q}
$$

and substituting above we get

$$
(q)_{\infty}^{n-2} p_{n, k}=p_{2, k}-\left(2^{n}-4\right)\left(\begin{array}{c}
2 k-1 \\
k
\end{array}\right)_{q} \sum_{i=k+1}^{2 k-n+3} q^{i}+q^{2 k-n+3}+\mathcal{O}\left(q^{2 k-n+4}\right) .
$$


Multiplying by $(q)_{\infty}$ and observing that $(q)_{\infty}\left(\begin{array}{c}2 k-1 \\ k\end{array}\right)_{q}=\left(q^{k}\right)_{k}\left(q^{k+1}\right)_{\infty}=1+\mathcal{O}\left(q^{k}\right)$ we get, since $n \geq 3$ :

$$
(q)_{\infty}^{n-1} p_{n, k}=(q)_{\infty} p_{2, k}-\left(2^{n}-4\right) \sum_{i=k+1}^{2 k-n+3} q^{i}+q^{2 k-n+3}+\mathcal{O}\left(q^{2 k-n+4}\right) .
$$

The result now easily follows.

Let $I \subset \mathbb{C}^{2}$ be a finite set with cardinality $\# I=n$ and let $\mathscr{C}_{I, k}=\mathscr{C}_{I, k}^{\mathbb{H}}$ denote the rank-stable limit of the space $\mathscr{C}_{I, k}^{\mathbb{V}}$ of equivalence classes of framed connections on an $S U(\mathbb{V})$ vector bundle over $\#_{n} \overline{\mathbb{P}}^{2}$ with $c_{2}=k$ (see section 9). The space $\mathscr{C}_{I, k}$ is homotopically equivalent to $B U^{n+1}$ (see [8, Remark 1.1]). We are ready to prove Theorem 1.6, which is a consequence of the following theorem:

Theorem [1.6*. Let $\imath_{k}: \mathfrak{M}_{I, k} \cong \mathfrak{M I}_{I, k} \rightarrow \mathscr{C}_{I, k}$ be the natural inclusion map and let $h_{I, k}:\left\|\mathfrak{B}_{I, k}\right\| \rightarrow \mathfrak{M}_{I, k}$ be the $c_{2}=k$ components of the map $h_{I}$ in Definition 5.2];

$$
\left\|\mathfrak{B}_{I, k}\right\| \stackrel{h_{I, k}}{\longrightarrow} \mathfrak{M}_{I, k} \cong \mathfrak{M} \mathcal{I}_{I, k} \stackrel{\imath_{k}}{\longrightarrow} \mathscr{C}_{I, k} .
$$

(1) The map $\imath_{k}$ induces surjective homomorphisms in homology and homotopy up to degree $2 k+1$.

(2) The map $h_{I, k}$ is injective in homology.

(3) The map $h_{I, k}$ is an isomorphism in homology in degree $i$ provided $i \leq 2 k+1$ and the map $\left(\imath_{k}\right)_{*}: H_{i}\left(\mathfrak{M} \mathcal{I}_{I, k}\right) \rightarrow H_{i}\left(\mathscr{C}_{I, k}\right)$ is an isomorphism.

(4) The map $h_{I, k}$ is an isomorphism in homotopy in degree $i$ provided $i \leq 2 k$ and the map $\left(\imath_{k}\right)_{*}: \pi_{i}\left(\mathfrak{M} \mathcal{I}_{I, k}\right) \rightarrow \pi_{i}\left(\mathscr{C}_{I, k}\right)$ is an isomorphism.

(5) Let $b_{i}$ be the Betti numbers of $B U^{n+1}$, let $c_{2 i}$ be the coefficients of the power series:

$$
\sum_{i=0}^{\infty} c_{2 i} q^{i}=\left(\sum_{i=0}^{\infty} b_{2 i} q^{i}\right)\left(2^{n}\left(q^{k+1}+\cdots+q^{2 k-n+2}\right)+\left(2^{n}-1\right) q^{2 k-n+3}\right),
$$

and set $c_{i}=0$ for $i$ odd. Then the rank of the cokernel of the map $\left(\imath_{k}\right)_{*}: H_{i}\left(\mathfrak{M I}_{I, k}\right) \rightarrow H_{i}\left(\mathscr{C}_{I, k}\right)$ is less than or equal to $c_{i}$ for $i \leq 4 k-2 n+7$, provided $2 \leq n \leq k+1$.

Proof. By Proposition 9.1 the inclusions $\mathfrak{M}_{I} \rightarrow \mathscr{C}_{I}$ are maps of $\mathscr{L}$-algebras which induce a map of the bar constructions

$$
\left\|\mathfrak{B}_{I}\right\|=\mathfrak{B}\left(\prod \mathfrak{M}_{x}, \prod \mathfrak{M}_{\emptyset}, \mathfrak{M}_{\emptyset}\right) \rightarrow \mathfrak{B}\left(\prod \mathscr{C}_{x}, \prod \mathscr{C}_{\emptyset}, \mathscr{C}_{\emptyset}\right)
$$

and we get a commutative diagram, where the right vertical map is a homotopy equivalence (Proposition 9.4):

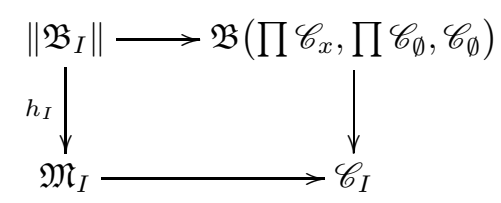

To prove (2) it is enough to show that the top horizontal map is injective in homology, which we now prove. Let $\bar{R}=H_{*}\left(\mathscr{C}_{\emptyset}\right) \cong H_{*}(B U \times \mathbb{Z})$ and $\bar{M}=H_{*}\left(\mathscr{C}_{x}\right) \cong$ $H_{*}(B U \times B U \times \mathbb{Z})$, and let $r_{0}$ be a generator of $H_{0}(\{1\} \times B U)$. Then $\bar{R}$ and 
$\bar{M}$ are respectively the localizations of $R$ and $M$ away from $r_{0}: \bar{R}=R\left[r_{0}^{-1}\right]$ and $\bar{M}=M\left[r_{0}^{-1}\right]$. Also $\bar{M}$ is free over $\bar{R}$ and:

$$
H_{*}\left(\mathfrak{B}\left(\prod \mathscr{C}_{x}, \prod \mathscr{C}_{\emptyset}, \mathscr{C}_{\emptyset}\right)\right) \cong \bar{M} \otimes_{\bar{R}} \cdots \otimes_{\bar{R}} \bar{M} \cong\left(M \otimes_{R} \cdots \otimes_{R} M\right)\left[r_{0}^{-1}\right] .
$$

Since $M \otimes_{R} \cdots \otimes_{R} M$ is $r_{0}$-torsion free, the map $M \otimes_{R} \cdots \otimes_{R} M \rightarrow\left(M \otimes_{R} \cdots \otimes_{R}\right.$ $M)\left[r_{0}^{-1}\right]$ is injective as claimed. It follows that the map $h_{I}$ is injective in homology.

We will now compute the dimension of the cokernel of the map $H_{*}\left(\left\|\mathfrak{B}_{I, k}\right\|\right) \rightarrow$ $H_{*}\left(\mathscr{C}_{I, k}\right)$ induced by the diagonal map in diagram (10.11). The ring $H_{*}\left(\mathscr{C}_{I, k}\right)$ is graded by half the homology degree with Hilbert series $\sum_{i} b_{2 i} q^{i}=\sum_{i} 1 /(q)_{\infty}^{n+1}$ and the Hilbert series of $H_{*}\left(\left\|\mathfrak{B}_{I, k}\right\|\right)$ was computed in Lemma 10.9. Subtracting the coefficients of the Hilbert series we obtain:

$\frac{1}{(q)_{\infty}^{n+1}}-\frac{p_{n, k}}{(q)_{k}^{2}}=\frac{1-\left(q^{k+1}\right)_{\infty}^{2}(q)_{\infty}^{n-1} p_{n, k}}{(q)_{\infty}^{n+1}}=\left(\sum_{i=0}^{\infty} b_{2 i} q^{i}\right)\left(1-\left(q^{k+1}\right)_{\infty}^{2}(q)_{\infty}^{n-1} p_{n, k}\right)$.

Statement (5) follows from Lemma 10.9 (3), observing that $\left(q^{k+1}\right)_{\infty}^{2}=1-2\left(q^{k+1}+\right.$ $\left.\cdots+q^{2 k+1}\right)+\mathcal{O}\left(q^{2 k+2}\right)$. Furthermore, by Lemma 10.9 $(2)$, the map $H_{*}\left(\left\|\mathfrak{B}_{I}\right\|\right) \rightarrow$ $H_{*}\left(\mathscr{C}_{I}\right)$ is an isomorphism in degrees up to $2 k+1$, proving (3). Since $\mathscr{C}_{I, k} \simeq B U^{n+1}$ and $\left\|\mathfrak{B}_{I, k}\right\|$ are simply connected (Proposition 10.8), by the Hurewicz Theorem the map $\pi_{*}\left(\left\|\mathfrak{B}_{I, k}\right\|\right) \rightarrow \pi_{*}\left(\mathscr{C}_{I, k}\right)$ is an isomorphism in degrees up to $2 k$ and is surjective in degree $2 k+1$. Statements (1) and (4) immediately follow.

\section{Appendix A. BAR CONSTRUCTION}

In this appendix we prove propositions 4.18 and 5.12. First we need to prove some lemmas. Let $\widetilde{\Delta}$ be as in Definition 4.12 The identity $M \times A^{n} \times A=M \times A^{n+1}$ leads us to make the following definition:

Definition A.1. We represent by $\bar{\Delta}$ and $\widehat{\Delta}$ the categories whose objects are the same as the objects of $\widetilde{\Delta}$ (with $\emptyset=[-1])$ and such that $\bar{\Delta}(m, n) \subset \Delta(m+1, n+1)$ is the set of order preserving maps $\bar{\mu}:\{0, \ldots, m+1\} \rightarrow\{0, \ldots, n+1\}$ with $\bar{\mu}(m+1)=$ $n+1$, and $\widehat{\Delta}(m, n)$ is the set of order preserving maps $\widehat{\mu}:\{-1,0, \ldots, m+1\} \rightarrow$ $\{-1,0, \ldots, n+1\}$ with $\widehat{\mu}(m+1)=n+1$ and $\widehat{\mu}(-1)=-1$.

Remark A.2. The categories $\Delta$ and $\widetilde{\Delta}$ are subcategories of $\bar{\Delta}$ since we can extend any function $[m] \rightarrow[n]$ canonically to a morphism $[m+1] \rightarrow[n+1]$ by sending $m+1$ to $n+1$. In a similar way, $\bar{\Delta}$ is a subcategory of $\widehat{\Delta}$. Also observe that the object $[-1]$ is a final object of both $\bar{\Delta}^{\mathrm{op}}$ and $\widehat{\Delta}^{\mathrm{op}}$ and, for $m \neq-1$, restriction gives an isomorphism of sets $\bar{\Delta}(m, n) \cong \Delta(m, n+1)$.

Lemma A.3. The inclusion functors $F: \Delta^{\mathrm{op}} \rightarrow \bar{\Delta}^{\mathrm{op}}, \widetilde{F}: \widetilde{\Delta}^{\mathrm{op}} \rightarrow \bar{\Delta}^{\mathrm{op}}$ and $\widehat{F}: \bar{\Delta}^{\mathrm{op}} \rightarrow \widehat{\Delta}^{\mathrm{op}}$ are right cofinal.

For the definition of right cofinal see equation (2.8).

Proof. We first show that $\widetilde{F}$ is right cofinal. It is enough to show that, for any $[n] \in \bar{\Delta}$, the overcategory $\widetilde{F} \downarrow[n]$ has a final object. This object is the pair $([n+1], \imath)$, where $[n+1] \in \widetilde{\Delta}$ and $\imath: F([n+1])=[n+1] \rightarrow[n]$ corresponds to the identity map under the isomorphism $\bar{\Delta}(n+1, n) \cong \Delta(n+1, n+1)=\widetilde{\Delta}(n+1, n+1)$. The exact same proof shows that $F$ is right cofinal since the pair $([n+1], \imath)$ is also an object in $F \downarrow[n]$. 
The proof for $\widehat{F}$ is similar. Let $f \in \widehat{\Delta}(n+1, n)$ be the map $f:\{-1, \ldots, n+2\} \rightarrow$ $\{-1, \ldots, n+1\}$ with $f(-1)=-1$ and $f(i)=i-1$ for $i=0, \ldots, n+1$. We will show that $([n+1], f)$ is a final object of $\widehat{F} \downarrow[n]$ : given a map $\widehat{\mu}:\{-1, \ldots, m+1\} \rightarrow$ $\{-1, \ldots, n+1\}$ there is a unique map $\bar{\mu}:\{0, \ldots, m+1\} \rightarrow\{0, \ldots, n+2\}$ such that $\widehat{\mu}=f \circ \widehat{F}(\bar{\mu})$, namely: $\bar{\mu}(i)=\widehat{\mu}(i)+1$. This completes the proof.

Let $\mathcal{P}$ be an $E_{\infty}$ operad with composition data $\gamma$ and let $\mathscr{M}$ be an $E_{\infty}$ monoidal module over $\mathcal{P}$ with composition data $\Gamma_{R}$ (see Definition 4.1).

Definition A.4. We denote by $\bar{\Delta}(\mathscr{M}, \mathcal{P})$ the topological category equivalent to $\bar{\Delta}$ whose morphisms are defined as follows: for each $\mu \in \Delta(m+1, n+1)$ let

$$
\bar{\Delta}_{\mu}(\mathscr{M}, \mathcal{P})=\mathscr{M}\left(\mu_{0}\right) \times \prod_{\alpha=1}^{m+1} \mathcal{P}\left(\mu_{\alpha}-\mu_{\alpha-1}\right)
$$

and define

$$
\bar{\Delta}(\mathscr{M}, \mathcal{P})(m, n)=\coprod_{\mu \in \bar{\Delta}(m, n)} \bar{\Delta}_{\mu}(\mathscr{M}, \mathcal{P}) .
$$

Given $\mu \in \bar{\Delta}(m, n)$ and $\nu \in \bar{\Delta}(n, p)$, composition $\bar{\Delta}_{\mu}(\mathscr{M}, \mathcal{P}) \times \bar{\Delta}_{\nu}(\mathscr{M}, \mathcal{P}) \rightarrow$ $\bar{\Delta}_{\nu \circ \mu}(\mathscr{M}, \mathcal{P})$ is defined using the operad data:

$$
\begin{aligned}
\mathscr{M}\left(\mu_{0}\right) \times \mathscr{M}\left(\nu_{0}\right) \times \prod_{\beta=1}^{\mu_{0}} \mathcal{P}\left(\nu_{\beta}-\nu_{\beta-1}\right) \stackrel{\Gamma_{R}}{\longrightarrow} \mathscr{M}\left(\nu_{\mu_{0}}\right) ; \\
\mathcal{P}\left(\mu_{\alpha}-\mu_{\alpha-1}\right) \times \prod_{\beta=\mu_{\alpha-1}+1}^{\mu_{\alpha}} \mathcal{P}\left(\nu_{\beta}-\nu_{\beta-1}\right) \stackrel{\gamma}{\rightarrow} \mathcal{P}\left(\nu_{\mu_{\alpha}}-\nu_{\mu_{\alpha-1}}\right) \quad(\alpha=1, \ldots, m+1) .
\end{aligned}
$$

Notice that, for $\mu_{m} \leq n$, we have $\mathcal{P}_{+}\left(n-\mu_{m}\right)=\mathcal{P}\left(\mu_{m+1}-\mu_{m}\right)$ hence $\Delta(\mathscr{M}, \mathcal{P})$ and $\widetilde{\Delta}(\mathscr{M}, \mathcal{P})$ (see Definitions 4.8 and 4.13) are canonically subcategories of $\bar{\Delta}(\mathscr{M}, \mathcal{P})$.

Lemma A.5. The functor $\widetilde{\mathfrak{B}} \bullet(M, A, A ; M)$ from Definition 4.13 (2) can be extended to a functor $\overline{\mathfrak{B}}_{\bullet}(M, A): \bar{\Delta}(\mathscr{M}, \mathcal{P})^{\mathrm{op}} \rightarrow$ Top.

Proof. Given $\mu \in \Delta(m+1, n+1)$ with $\mu_{m+1}=n+1$, the required maps

$$
\bar{\Delta}_{\mu}(\mathscr{M}, \mathcal{P}) \times M \times A^{n+1}=\bar{\Delta}_{\mu}(\mathscr{M}, \mathcal{P}) \times M \times A^{\mu_{0}} \times \prod_{\alpha=1}^{m+1} A^{\mu_{\alpha}-\mu_{\alpha-1}} \rightarrow M \times A^{m+1}
$$

are induced by the maps

$$
\begin{aligned}
& \mathscr{M}\left(\mu_{0}\right) \times M \times A^{\mu_{0}} \rightarrow M ; \\
& \mathcal{P}\left(\mu_{\alpha}-\mu_{\alpha-1}\right) \times A^{\mu_{\alpha}-\mu_{\alpha-1}} \rightarrow A .
\end{aligned}
$$

Let $\overline{\mathfrak{B}}(M, A)$ be the homotopy colimit of the functor $\overline{\mathfrak{B}} \bullet(M, A)$ in Lemma A.5 Proposition 4.18 is now a direct consequence of the following lemma:

Lemma A.6. We have a commutative diagram

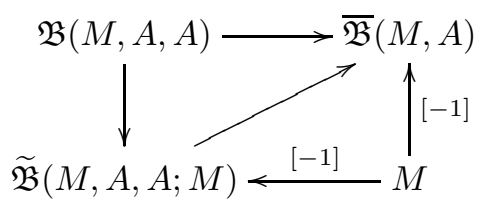

where every map is a homotopy equivalence. 
Proof. The bottom horizontal map is a homotopy equivalence by Proposition 4.16 so it is enough to show that the inclusion functor $\Delta(\mathscr{M}, \mathcal{P})^{\text {op }} \rightarrow \bar{\Delta}(\mathscr{M}, \mathcal{P})^{\text {op }}$ and the functor $\star \rightarrow \bar{\Delta}(\mathscr{M}, \mathcal{P})^{\text {op }}$ which sends $\star$ to $[-1]$ are right cofinal. This follows from the pullback squares
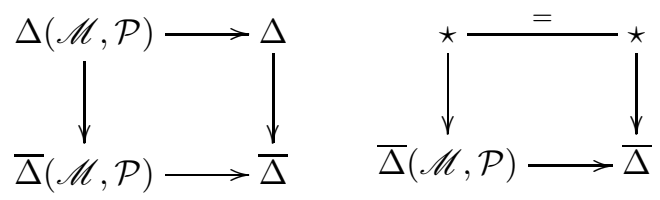

since the horizontal maps are equivalences of categories and the right vertical maps are cofinal.

We now turn to the proof of Proposition 5.12, Recall Definitions 3.1 and 5.1 Given disjoint finite sets $I, J \subset \mathbb{C}^{2}$, a universe $\mathbb{H}$ and integers $a, b, c$ with $a \geq 1$ and $b, c \geq 0$ let

$$
\mathscr{L}_{I J}^{\mathbb{H}}(a, b, c)=\mathscr{I}\left(\mathbb{H}^{a} \oplus\left(\mathbb{H}^{I}\right)^{b} \oplus\left(\mathbb{H}^{J}\right)^{c}, \mathbb{H}\right) .
$$

By Proposition $3.7, \mathfrak{M}_{\emptyset}$ is an $\mathscr{I}$-functor so we have maps

$$
\begin{aligned}
\mathscr{L}_{I J}^{\mathbb{H}}(a, b, c) \times\left(\mathfrak{M}_{\emptyset}^{\mathbb{H}}\right)^{a} \times\left(\prod_{I} \mathfrak{M}_{\emptyset}^{\mathbb{H}}\right)^{b} & \times\left(\prod_{J} \mathfrak{M}_{\emptyset}^{\mathbb{H}}\right)^{c} \\
\rightarrow \mathscr{L}_{I J}^{\mathbb{H}}(a, b, c) \times \mathfrak{M}_{\emptyset}^{\mathbb{H}^{a}} \oplus\left(\mathbb{H}^{I}\right)^{b} \oplus\left(\mathbb{H}^{J}\right)^{c} & \rightarrow \mathfrak{M}_{\emptyset}^{\mathbb{H}} .
\end{aligned}
$$

Note that $\mathscr{L}_{I J}^{\mathbb{H}}(1,0, c)=\mathscr{L}_{J}^{\mathbb{H}}(c)$ and $\mathscr{L}_{I J}^{\mathbb{H}}(1, b, 0)=\mathscr{L}_{I}^{\mathbb{H}}(b)$. We also have an isomorphism $\mathscr{L}_{I J}^{\mathbb{H}}(1, a, a) \cong \mathscr{L}_{I \cup J}^{\mathbb{H}}(a)$ induced by the permutation

$$
\tau: \mathbb{H} \oplus\left(\mathbb{H}^{I}\right)^{a} \oplus\left(\mathbb{H}^{J}\right)^{a} \rightarrow \mathbb{H} \oplus\left(\mathbb{H}^{I \cup J}\right)^{a} .
$$

The permutation $\tau$ also induces a map

$$
\tau: \mathfrak{M}_{\emptyset}^{\mathbb{H}} \times\left(\prod_{I} \mathfrak{M}_{\emptyset}^{\mathbb{H}}\right)^{a} \times\left(\prod_{J} \mathfrak{M}_{\emptyset}^{\mathbb{H}}\right)^{a} \rightarrow \mathfrak{M}_{\emptyset}^{\mathbb{H}} \times\left(\prod_{I \cup J} \mathfrak{M}_{\emptyset}^{\mathbb{H}}\right)^{a}
$$

and we have a commutative diagram:

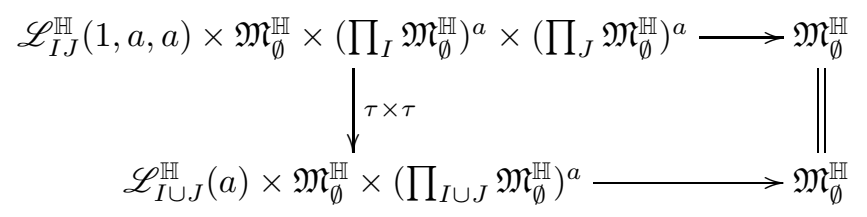

Proposition 5.12, Let $I, J \subset \mathbb{C}^{2}$ be finite disjoint sets. Fix universes $\mathbb{H}_{I J}, \mathbb{H}_{\emptyset}$ and let $\mathbb{H}=\mathbb{H}_{\emptyset} \otimes \mathbb{H}_{I J}$. Let $i: \mathscr{L}^{\mathbb{H}_{I J}} \rightarrow \mathscr{L}^{\mathbb{H}}$ be the canonical operad map. Then, in hTop, we have a commutative diagram:

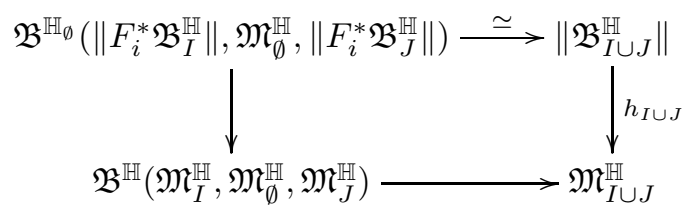

where the top horizontal map is a homotopy equivalence and the left vertical map is induced by $h_{I}$ and $h_{J}$. 
Proof. As in the proof of Proposition 8.42, the strategy is to compare the bar construction with a trisimplicial version and take the diagonal. In the proof of 8.42 we only needed two functors: $F_{0}$ and $F_{1}$. Here, however, we need 3 more functors to define the vertical maps in diagram (A.9) (see section 4.3). Recall the functors in equation (5.11). We will define diagrams of spaces (see section 2.1.1) $\mathfrak{F}_{0}, \mathfrak{F}_{1}, \widehat{\mathfrak{F}}_{1}$, $\mathfrak{F}_{2}, \mathfrak{F}_{3}$ indexed by certain categories $C_{0}, C_{1}, \widehat{C}_{1}, C_{2}, C_{3}$ in such a way that we get a commutative diagram in hTop (compare with diagram 8.43):

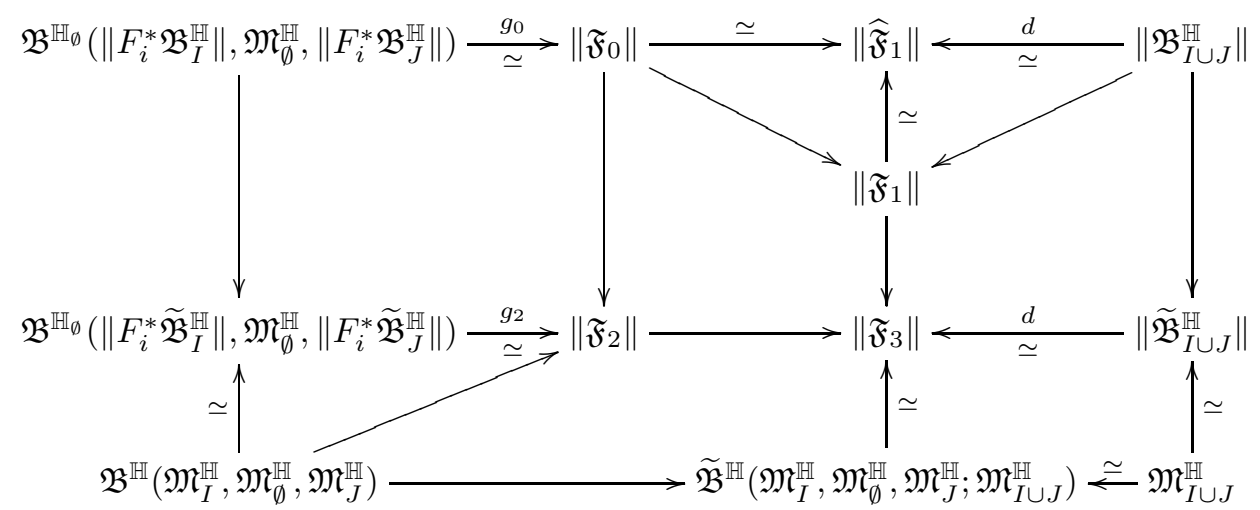

The result then immediately follows.

We begin by defining a category $C$ topologically equivalent to $\widetilde{\Delta} \times \widehat{\Delta} \times \widetilde{\Delta}$ (see Definition A.1). The categories $C_{0}, C_{1}, \widehat{C}_{1}, C_{2}, C_{3}$ will be subcategories of $C$. The objects of $C$ are the triples $\left(m^{I}, m, m^{J}\right)$ of integers with $m^{I}, m, m^{J} \geq-1$. To define the morphisms recall the notation of Definition 5.1. Given morphisms $\mu^{I} \in \widetilde{\Delta}\left(m^{I}, n^{I}\right), \mu \in \widehat{\Delta}(m, n)$ and $\mu^{J} \in \widetilde{\Delta}\left(m^{J}, n^{J}\right)$, with $m \neq-1$, let

$$
\begin{aligned}
C\left(\mu^{I}, \mu, \mu^{J}\right)= & \left(\prod_{\alpha=1}^{m^{I}+1} \mathscr{L}^{\mathbb{H}, I}\left(\mu_{\alpha}^{I}-\mu_{\alpha-1}^{I}\right)\right) \\
& \times \mathscr{L}_{I J}^{\mathbb{H}}\left(1+\mu_{0}, \mu_{0}^{I}, 0\right) \times\left(\prod_{\alpha=1}^{m} \mathscr{L}^{\mathbb{H}}\left(\mu_{\alpha}-\mu_{\alpha-1}\right)\right) \\
& \quad \times \mathscr{L}_{I J}^{\mathbb{H}}\left(1+n-\mu_{m}, 0, \mu_{0}^{J}\right) \times\left(\prod_{\alpha=1}^{m^{J}+1} \mathscr{L}^{\mathbb{H}, J}\left(\mu_{\alpha}^{J}-\mu_{\alpha-1}^{J}\right)\right),
\end{aligned}
$$

(with the convention that $\mu_{m^{I}+1}^{I}=n^{I}+1$ and $\mu_{m^{J}+1}^{J}=n^{J}+1$ ). For $m=-1$ let

$$
\begin{aligned}
C\left(\mu_{I}, \imath, \mu_{J}\right)=\left(\prod_{\alpha=1}^{m^{I}+1} \mathscr{L}^{\mathbb{H}, I}\left(\mu_{\alpha}^{I}-\mu_{\alpha-1}^{I}\right)\right) & \\
& \times \mathscr{L}_{I J}\left(2+n, \mu_{0}^{I}, \mu_{0}^{J}\right) \times\left(\prod_{\alpha=1}^{m^{J}+1} \mathscr{L}^{\mathbb{H}, J}\left(\mu_{\alpha}^{J}-\mu_{\alpha-1}^{J}\right)\right)
\end{aligned}
$$

where $\imath$ is the unique morphism in $\widehat{\Delta}(-1, n)$. We define the morphisms in $C$ by

$$
C\left(\left(m^{I}, m, m^{J}\right),\left(n^{I}, n, n^{J}\right)\right)=\coprod_{\mu^{I}, \mu, \mu^{J}} C\left(\mu^{I}, \mu, \mu^{J}\right) .
$$


We define the categories $\widehat{C}_{1}$ and $C_{3}$ by the pullback diagrams

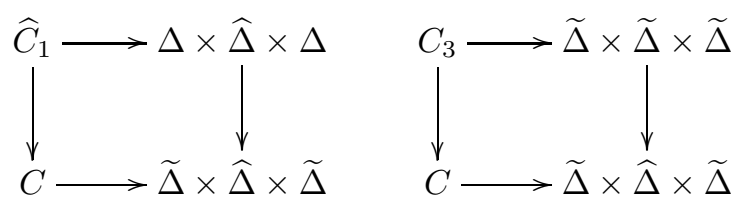

Also let $C_{2} \subset C_{3}$ be the full subcategory whose objects are the triples of integers $\left(n^{I}, n, n^{J}\right)$ with $n^{I}, n^{J} \geq-1$ and $n \geq 0$, let $C_{1}=C_{3} \cap \widehat{C}_{1}$ and let $C_{0}=C_{2} \cap C_{1}$. Notice that we have equivalences of categories $C_{2} \simeq \widetilde{\Delta} \times \Delta \times \widetilde{\Delta}, C_{1} \simeq \Delta \times \widetilde{\Delta} \times \Delta$ and $C_{0} \simeq \Delta \times \Delta \times \Delta$.

We now define the functor $\mathfrak{F}_{2}: C_{2}^{\text {op }} \rightarrow \underline{\text { Top. }}$. On objects, $\mathfrak{F}_{2}\left(n^{I}, n, n^{J}\right)=\widetilde{\mathfrak{B}}_{I}^{\mathbb{H}}\left(n^{I}\right) \times$ $\left(\mathfrak{M}_{\emptyset}^{\mathbb{H}}\right)^{n} \times \widetilde{\mathfrak{B}}_{J}^{\mathbb{H}}\left(n^{J}\right)$ and the functor $\mathfrak{F}_{2}$ is defined on morphisms as follows: given morphisms $\mu^{I} \in \widetilde{\Delta}\left(m^{I}, n^{I}\right), \mu \in \Delta(m, n), \mu^{J} \in \widetilde{\Delta}\left(m^{J}, n^{J}\right)$, and $f \in C\left(\mu^{I}, \mu, \mu^{J}\right)$, we can write, for $n^{I}, n^{J} \neq-1$,

$$
\begin{aligned}
& \mathfrak{F}_{2}\left(n^{I}, n, n^{J}\right) \\
& \stackrel{\text { shuffle }}{\longrightarrow}\left(\prod_{I} \mathfrak{M}_{\emptyset}^{\mathbb{H}}\right)^{n^{I}-\mu_{0}^{I}} \times \prod_{I} \mathfrak{M}_{x}^{\mathbb{H}} \times\left(\left(\mathfrak{M}_{\emptyset}^{\mathbb{H}}\right)^{1+\mu_{0}} \times\left(\prod_{I} \mathfrak{M}_{\emptyset}^{\mathbb{H}}\right)^{\mu_{0}^{I}}\right) \times\left(\mathfrak{M}_{\emptyset}^{\mathbb{H}}\right)^{n-\mu_{0}} \times \widetilde{\mathfrak{B}}_{J}^{\mathbb{H}}\left(n^{J}\right) \\
& \stackrel{f}{\rightarrow} \mathfrak{F}_{2}\left(m^{I}, m, m^{J}\right)
\end{aligned}
$$

where $f$ acts on the product as in Definition 4.10 and equation (A.7). If either $n^{I}$ or $n^{J}$ equal $-1, f$ acts using the structure of $\mathfrak{M}_{I}^{\mathbb{H}}$ and $\mathfrak{M}_{J}^{\mathbb{H}}$ as modules over the $\mathscr{L}^{\mathbb{H}}$-algebra $\mathfrak{M}_{\emptyset}^{\mathbb{H}}$ (Remark 3.11).

We define the functor $\mathfrak{F}_{0}$ as the restriction of $\mathfrak{F}_{2}$ to $C_{0}^{\text {op }}$; then the inclusion $C_{0} \subset C_{2}$ induces a map $\left\|\mathfrak{F}_{0}\right\| \rightarrow\left\|\mathfrak{F}_{2}\right\|$.

We now define the homotopy equivalences $g_{0}$ and $g_{2}$. Let $\Delta_{0}^{\mathbb{H}_{\emptyset}}=\Delta\left(\mathscr{L}_{+}^{\mathbb{H}_{\emptyset}}, \mathscr{L}^{\mathbb{H}_{\emptyset}}\right)$ (see Definition 4.8) and consider the functor $F: \widetilde{\Delta}_{I}^{\mathbb{H}_{I J}} \times \Delta_{0}^{\mathbb{H}_{\emptyset}} \times \widetilde{\Delta}_{J}^{\mathbb{H}_{I J}} \rightarrow C_{2}$ (see Definition (5.2) which is the identity on objects and is induced on morphisms as folows: given morphisms $\mu^{I} \in \widetilde{\Delta}\left(m^{I}, n^{I}\right), \mu \in \Delta(m, n)$ and $\mu^{J} \in \widetilde{\Delta}\left(m^{J}, n^{J}\right)$, the map

$$
\widetilde{\Delta}_{I}^{\mathbb{H}_{I J}} \times \Delta_{0}^{\mathbb{H}_{\emptyset}} \times \widetilde{\Delta}_{J}^{\mathbb{H}_{I J}} \rightarrow C_{2}
$$

is given by the maps

$$
\begin{aligned}
& \prod_{\alpha=1}^{m^{I}+1} \mathscr{L}^{\mathbb{H}_{I J}, I}\left(\mu_{\alpha}^{I}-\mu_{\alpha-1}^{I}\right) \rightarrow \prod_{\alpha=1}^{m^{I}+1} \mathscr{L}^{\mathbb{H}, I}\left(\mu_{\alpha}^{I}-\mu_{\alpha-1}^{I}\right) \\
& \prod_{\alpha=1}^{m+1} \mathscr{L}^{\mathbb{H}_{\emptyset}}\left(\mu_{\alpha}-\mu_{\alpha-1}\right) \rightarrow \prod_{\alpha=1}^{m+1} \mathscr{L}^{\mathbb{H}}\left(\mu_{\alpha}-\mu_{\alpha-1}\right) \\
& \prod_{\alpha=1}^{m^{J}+1} \mathscr{L}^{\mathbb{H}_{I J}, J}\left(\mu_{\alpha}^{J}-\mu_{\alpha-1}^{J}\right) \rightarrow \prod_{\alpha=1}^{m^{J}+1} \mathscr{L}^{\mathbb{H}, J}\left(\mu_{\alpha}^{J}-\mu_{\alpha-1}^{J}\right)
\end{aligned}
$$

induced by the the canonical maps $i: \mathscr{L}^{\mathbb{H}_{I J}} \rightarrow \mathscr{L}^{\mathbb{H}}$ and $i_{\emptyset}: \mathscr{L}^{\mathbb{H}_{\emptyset}} \rightarrow \mathscr{L}^{\mathbb{H}}$, and by maps

$$
\begin{aligned}
\mathscr{L}_{I}^{\mathbb{H}_{I J}}\left(\mu_{0}^{I}\right) \times \mathscr{L}^{\mathbb{H}_{\emptyset}}\left(1+\mu_{0}\right) & \rightarrow \mathscr{L}_{I J}^{\mathbb{H}}\left(1+\mu_{0}, \mu_{0}^{I}, 0\right) \\
\mathscr{L}^{\mathbb{H}_{\emptyset}}\left(1+n-\mu_{m}\right) \times \mathscr{L}_{J}^{\mathbb{H}_{I J}}\left(\mu_{0}^{J}\right) & \rightarrow \mathscr{L}_{I J}^{\mathbb{H}}\left(1+n-\mu_{m}, 0, \mu_{0}^{J}\right)
\end{aligned}
$$


which we now define. Using matrix notation, the image by the first map of a pair of isometries

$$
\left[\begin{array}{ll}
g_{I J} & h_{I J}
\end{array}\right]: \mathbb{H}_{I J} \oplus\left(\left(\mathbb{H}_{I J}\right)^{I}\right)^{\mu_{0}^{I}} \rightarrow \mathbb{H}_{I J}, \quad\left[\begin{array}{ll}
g_{\emptyset} & f_{\emptyset}
\end{array}\right]: \mathbb{H}_{\emptyset} \oplus\left(\mathbb{H}_{\emptyset}\right)^{\mu_{0}} \rightarrow \mathbb{H}_{\emptyset}
$$

is the isometry

$$
\left[g_{\emptyset} \otimes g_{I J} \quad f_{\emptyset} \otimes g_{I J} \quad g_{\emptyset} \otimes h_{I J}\right]: \mathbb{H} \oplus \mathbb{H}^{\mu_{0}} \oplus\left(\mathbb{H}^{I}\right)^{\mu_{0}^{I}} \rightarrow \mathbb{H},
$$

where we identified $\mathbb{H}^{\mu_{0}}$ with $\left(\mathbb{H}_{\emptyset}\right)^{\mu_{0}} \otimes \mathbb{H}_{I J}$ and $\left(\mathbb{H}^{I}\right)^{\mu_{0}^{I}}$ with $\mathbb{H}_{\emptyset} \otimes\left(\left(\mathbb{H}_{I J}\right)^{I}\right)^{\mu_{0}^{I}}$. The second map is defined in an analogous way: the image of a pair of isometries

$$
\left[\begin{array}{ll}
g_{\emptyset} & f_{\emptyset}
\end{array}\right]: \mathbb{H}_{\emptyset} \oplus\left(\mathbb{H}_{\emptyset}\right)^{n-\mu_{m}} \rightarrow \mathbb{H}_{\emptyset}, \quad\left[\begin{array}{ll}
g_{I J} & h_{I J}
\end{array}\right]: \mathbb{H}_{I J} \oplus\left(\left(\mathbb{H}_{I J}\right)^{J}\right)^{\mu_{0}^{J}} \rightarrow \mathbb{H}_{I J}
$$

is the isometry

$$
\left[g_{\emptyset} \otimes g_{I J} \quad f_{\emptyset} \otimes g_{I J} \quad g_{\emptyset} \otimes h_{I J}\right]: \mathbb{H} \oplus \mathbb{H}^{n-\mu_{m}} \oplus\left(\mathbb{H}^{J}\right)^{\mu_{0}^{J}} \rightarrow \mathbb{H} .
$$

Now, a direct verification shows that we have a homeomorphism of $\Delta_{0}^{\mathbb{H}_{\emptyset}}$-diagrams

$$
\underset{\left(\widetilde{\Delta}_{I}^{\mathbb{H}_{I} J} \times \widetilde{\Delta}_{J}^{\mathbb{H}_{I} J}\right)^{\mathrm{op}}}{\operatorname{hcol}} F^{*} \mathfrak{F}_{2}^{\mathbb{H}} \cong \mathfrak{B}_{\bullet}^{\mathbb{H}_{\emptyset}}\left(\left\|F_{i}^{*} \widetilde{\mathfrak{B}}_{I}^{\mathbb{H}}\right\|, \mathfrak{M}_{\emptyset}^{\mathbb{H}},\left\|F_{i}^{*} \widetilde{\mathfrak{B}}_{J}^{\mathbb{H}}\right\|\right),
$$

and since $F$ is an equivalence of categories, by [23, Proposition 3.1(6)] and the commutation of homotopy colimits (see [23, section 6]), we get

$$
\begin{aligned}
\underset{C_{2}^{\mathrm{op}}}{\operatorname{hcolim}} \mathfrak{F}_{2} & \underset{\left(\widetilde{\Delta}_{I}^{\mathbb{H}_{I} J} \times \Delta_{0}^{\mathbb{H}_{\emptyset}} \times \Delta_{J}^{\mathbb{H}_{I} J}\right)^{\mathrm{op}}}{\operatorname{hcolim}} F^{*} \mathfrak{F}_{2} \\
& \cong \underset{\left(\Delta_{0}^{\mathbb{H}}\right)^{\mathrm{op}}}{\operatorname{hcolim}}(\overbrace{\left(\widetilde{\Delta}_{I}^{\mathbb{H}_{I} J} \times \Delta_{J}^{\mathbb{H}_{I} J}\right)^{\mathrm{op}}} F^{*} \mathfrak{F}_{2}) \\
& \cong \underset{\left(\Delta_{0}^{\mathbb{H}_{\emptyset}}\right)^{\mathrm{op}}}{\operatorname{hcolim}} \mathfrak{B}_{\bullet}^{\mathbb{H}_{\emptyset}}\left(\left\|F_{i}^{*} \widetilde{\mathfrak{B}}_{I}^{\mathbb{H}}\right\|, \mathfrak{M}_{\emptyset}^{\mathbb{H}},\left\|F_{i}^{*} \widetilde{\mathfrak{B}}_{J}^{\mathbb{H}}\right\|\right)
\end{aligned}
$$

Therefore we have a commutative diagram in Top:

(A.10)

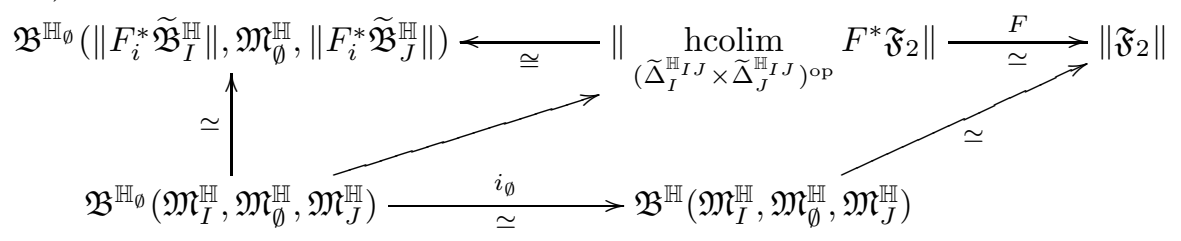

where the right diagonal map is induced by the functor $\Delta_{0}^{\mathbb{H}} \rightarrow C_{2}$ which sends $n$ to $(-1, n,-1)$. We define $g_{2}$ as the top row in diagram A.10, The map $g_{0}$ is defined by taking the restriction of $g_{2}$ to $\mathfrak{B}^{\mathbb{H}_{\emptyset}}\left(\left\|F_{i}^{*} \mathfrak{B}_{I}^{\mathbb{H}}\right\|, \mathfrak{M}_{\emptyset}^{\mathbb{H}},\left\|F_{i}^{*} \mathfrak{B}_{J}^{\mathbb{H}}\right\|\right)$ and observing that it factors through $\left\|\mathfrak{F}_{0}\right\|$.

We now construct functors $\widehat{\mathfrak{F}}_{1}: \widehat{C}_{1}^{\text {op }} \rightarrow \underline{\text { Top }}$ and $\mathfrak{F}_{3}: C_{3}^{\text {op }} \rightarrow \underline{\text { Top. }}$. On objects $\mathfrak{F}_{3}\left(n^{I}, n, n^{J}\right)=\mathfrak{F}_{2}\left(n^{I}, n, n^{J}\right)$ for $n \neq-1$,

$$
\mathfrak{F}_{3}\left(n^{I},-1, n^{J}\right)= \begin{cases}\widetilde{\mathfrak{B}}_{I}^{\mathbb{H}}\left(n^{I}\right) \times\left(\prod_{J} \mathfrak{M}_{\emptyset}^{\mathbb{H}}\right)^{n^{J}} \times\left(\prod_{x \in J} \mathfrak{M}_{x}^{\mathbb{H}}\right), & \text { if } n^{J} \neq-1 ; \\ \left(\prod_{x \in I} \mathfrak{M}_{x}^{\mathbb{H}}\right) \times\left(\prod_{I} \mathfrak{M}_{\emptyset}^{\mathbb{H}}\right)^{n^{I}} \times \widetilde{\mathfrak{B}}_{J}^{\mathbb{H}}\left(n^{J}\right) & \text { if } n^{I} \neq-1\end{cases}
$$

and $\mathfrak{F}_{3}(-1,-1,-1)=\mathfrak{M}_{I \cup J}^{\mathbb{H}}$. On objects, the functor $\widehat{\mathfrak{F}}_{1}$ is defined by $\widehat{\mathfrak{F}}_{1}\left(n^{I}, n, n^{J}\right)=$ $\mathfrak{F}_{3}\left(n^{I}, n, n^{J}\right)$ for $\left(n^{I}, n, n^{J}\right) \in \widehat{C}_{1}$. The functors are defined on morphisms in the usual way. We also define $\mathfrak{F}_{1}$ as the restriction of $\mathfrak{F}_{3}$ to $C_{1}^{\text {op }}$ (which coincides with 
the restriction of $\widehat{\mathfrak{F}}_{1}$ to $C_{1}^{\text {op }}$ ). Since, by Lemma A.3 the inclusions $\widetilde{\Delta} \rightarrow \widehat{\Delta}$ and $\bar{\Delta} \rightarrow \widehat{\Delta}$ are right cofinal, it follows that the inclusions $C_{0} \rightarrow \widehat{C}_{1}$ and $C_{1} \rightarrow \widehat{C}_{1}$ are also right cofinal and hence the maps $\left\|\mathfrak{F}_{0}\right\| \rightarrow\left\|\widehat{\mathfrak{F}}_{1}\right\|$ and $\left\|\mathfrak{F}_{1}\right\| \rightarrow\left\|\widehat{\mathfrak{F}}_{1}\right\|$ are homotopy equivalences.

The map $\widetilde{\mathfrak{B}}^{\mathbb{H}}\left(\mathfrak{M}_{I}^{\mathbb{H}}, \mathfrak{M}_{\emptyset}^{\mathbb{H}}, \mathfrak{M}_{J}^{\mathbb{H}} ; \mathfrak{M}_{I \cup J}^{\mathbb{H}}\right) \rightarrow\left\|\mathfrak{F}_{3}\right\|$ is induced by the functor $\widetilde{\Delta} \rightarrow$ $\widetilde{\Delta} \times \widetilde{\Delta} \times \widetilde{\Delta}$ given on objects by $n \mapsto(-1, n,-1)$ (compare with the right diagonal map in diagram A.10).

We now define a diagonal functor $d: \widetilde{\Delta}_{I \cup J} \rightarrow C_{3}$, given on objects by $n \mapsto$ $(n,-1, n)$; to define $d$ on morphisms just observe that, for any $\mu \in \widetilde{\Delta}(m, n)$, the spaces of morphisms $\widetilde{\Delta}_{I \cup J}(\mu)$ and $C_{3}(\mu, \mathbf{1}, \mu)$ are isomorphic (see diagram (A.8)). Now, direct inspection shows that $\widetilde{\mathfrak{B}}_{I \cup J}=d^{*} \mathfrak{F}_{3}$. Restricting $d$ we get a functor $d: \Delta_{I \cup J} \rightarrow C_{1}$ and we also have $\mathfrak{B}_{I \cup J}=d^{*} \mathfrak{F}_{1}$. We claim that the map $\left\|d^{*} \mathfrak{F}_{1}\right\| \stackrel{d}{\rightarrow}$ $\left\|\widehat{\mathfrak{F}}_{1}\right\|$ induced by $d$ and the inclusion $\mathfrak{F}_{1} \rightarrow \widehat{\mathfrak{F}}_{1}$ is a homotopy equivalence. This follows from the pullback rectangle in the diagram:

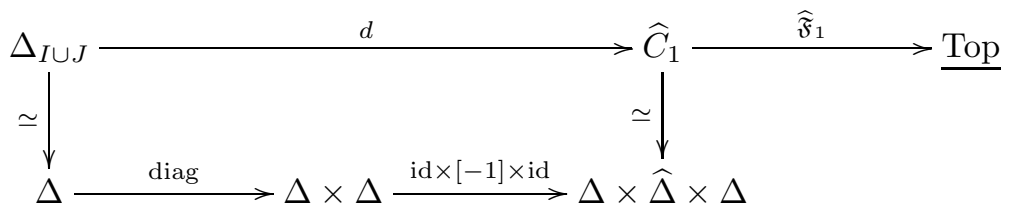

Since the bottom arrows are cofinal and the vertical arrows are equivalences, it follows that $d$ is cofinal and hence $\left\|d^{*} \mathfrak{F}_{1}\right\| \stackrel{d}{\rightarrow}\left\|\widehat{\mathfrak{F}}_{1}\right\|$ is a homotopy equivalence. In an analogous way we can see that the map $\left\|d^{*} \mathfrak{F}_{3}\right\| \stackrel{d}{\rightarrow}\left\|\mathfrak{F}_{3}\right\|$ is a homotopy equivalence, which finishes the proof.

Aknowledgements. The author would like to thank the referee for his careful reading of the manuscript and his comments, which greatly improved the readability of the text, and for pointing out that the proof of Theorem 1.1 was incomplete. The author would also like to thank Gustavo Granja for innumerous very fruitful discussions.

\section{REFERENCES}

1. Vigleik Angeltveit, The cyclic bar construction on $A_{\infty} H$-spaces, Adv. Math. 222 (2009), no. $5,1589-1610$.

2. M. F. Atiyah and J. Jones, Topological aspects of Yang-Mills theory, Comm. Math. Phys. 61 (1978), no. 2, 97-118.

3. Clemens Berger and Ieke Moerdijk, On the derived category of an algebra over an operad, Georgian Math. J. 16 (2009), no. 1, 13-28.

4. Bruce C. Berndt, What is a q-series?, Ramanujan rediscovered, Ramanujan Math. Soc. Lect. Notes Ser., vol. 14, Ramanujan Math. Soc., Mysore, 2010, pp. 31-51.

5. J. M. Boardman and R. M. Vogt, Homotopy-everything H-spaces, Bull. Amer. Math. Soc. 74 (1968), 1117-1122.

6. Charles P. Boyer, J. C. Hurtubise, B. M. Mann, and R. James Milgram, The topology of instanton moduli spaces. I. The Atiyah-Jones conjecture, Ann. of Math. (2) 137 (1993), no. 3, 561-609.

7. Charles P. Boyer and Benjamin M. Mann, Homology operations on instantons, J. Differential Geom. 28 (1988), no. 3, 423-465. MR 965223

8. J. Bryan and M. Sanders, The rank stable topology of instantons of $\overline{\mathbf{C P}}^{2}$, Proc. Amer. Math. Soc. 125 (1997), no. 12, 3763-3768.

9. _ Instantons on $S^{4}$ and $\overline{\mathbf{C P}}^{2}$, rank stabilization, and Bott periodicity, Topology 39 (2000), no. 2, 331-352. 
10. N. Buchdahl, Instantons on $n \mathbb{C P}^{2}$, J. Differential Geom. 37 (1993), no. 3, 669-687.

11. _ Blowups and gauge fields, Pacific J. Math. 196 (2000), no. 1, 69-111.

12. _ Monads and bundles on rational surfaces, Rocky Mountain J. Math. 34 (2004), no. 2, 513-540.

13. Ralph L. Cohen and R. James Milgram, The homotopy type of gauge-theoretic moduli spaces, Algebraic topology and its applications, Math. Sci. Res. Inst. Publ., vol. 27, Springer, New York, 1994, pp. 15-55. MR 1268186

14. S. K. Donaldson, Instantons and geometric invariant theory, Comm. Math. Phys. 93 (1984), no. 4, 453-460.

15. Connections, cohomology and the intersection forms of 4-manifolds, J. Differential Geom. 24 (1986), no. 3, 275-341.

16. S. K. Donaldson and P. Kronheimer, The geometry of four-manifolds, The Clarendon Press Oxford University Press, New York, 1990, Oxford Science Publications.

17. Emmanuel Dror Farjoun, Fundamental group of homotopy colimits, Adv. Math. 182 (2004), no. 1, 1-27. MR 2028495

18. Benoit Fresse, Modules over operads and functors, Lecture Notes in Mathematics, vol. 1967, Springer-Verlag, Berlin, 2009.

19. R. Friedman, Algebraic surfaces and holomorphic vector bundles, Springer-Verlag, New York, 1998.

20. Elizabeth Gasparim, The Atiyah-Jones conjecture for rational surfaces, Adv. Math. 218 (2008), no. 4, 1027-1050.

21. Victor Ginzburg and Mikhail Kapranov, Koszul duality for operads, Duke Math. J. 76 (1994), no. $1,203-272$.

22. Amar Abdelmoubine Henni, Monads for framed torsion-free sheaves on multi-blow-ups of the projective plane, Internat. J. Math. 25 (2014), no. 1, 1450008, 42.

23. J. Hollender and R. Vogt, Modules of topological spaces, applications to homotopy limits and $E_{\infty}$ structures, Arch. Math. (Basel) 59 (1992), no. 2, 115-129.

24. J. C. Hurtubise and R. James Milgram, The Atiyah-Jones conjecture for ruled surfaces, J. Reine Angew. Math. 466 (1995), 111-143.

25. D. Huybrechts and M. Lehn, Stable pairs on curves and surfaces, J. Algebraic Geom. 4 (1995), no. $1,67-104$.

26. A. King, Instantons and holomorphic bundles on the blown-up plane, Ph.D. thesis, Worcester College, Oxford, 1989.

27. Frances Clare Kirwan, Cohomology of quotients in symplectic and algebraic geometry, Mathematical Notes, vol. 31, Princeton University Press, Princeton, NJ, 1984.

28. - Geometric invariant theory and the Atiyah-Jones conjecture, The Sophus Lie Memorial Conference (Oslo, 1992), Scand. Univ. Press, Oslo, 1994, pp. 161-186.

29. Shoshichi Kobayashi, Differential geometry of complex vector bundles, Publications of the Mathematical Society of Japan, vol. 15, Princeton University Press, Princeton, NJ; Princeton University Press, Princeton, NJ, 1987, Kanô Memorial Lectures, 5.

30. M. Lübke, The analytic moduli space of framed vector bundles, J. Reine Angew. Math. 441 (1993), 45-59.

31. Martin Markl, Steve Shnider, and Jim Stasheff, Operads in algebra, topology and physics, Mathematical Surveys and Monographs, vol. 96, American Mathematical Society, Providence, RI, 2002.

32. Andreas Matuschke, On framed instanton bundles and their deformations, Math. Nachr. 211 (2000), 109-126.

33. J. P. May, Geometry of iterated loop spaces, Lecture notes in mathematics, Springer, 1972.

34. — $E_{\infty}$ ring spaces and $E_{\infty}$ ring spectra, Springer-Verlag, Berlin, 1977, With contributions by Frank Quinn, Nigel Ray, and Jørgen Tornehave, Lecture Notes in Mathematics, Vol. 577.

35. J. P. May and K. Ponto, More concise algebraic topology, Chicago Lectures in Mathematics, University of Chicago Press, Chicago, IL, 2012, Localization, completion, and model categories.

36. John W. Morgan, Tomasz Mrowka, and Daniel Ruberman, The $L^{2}$-moduli space and a vanishing theorem for Donaldson polynomial invariants, Monographs in Geometry and Topology, II, International Press, Cambridge, MA, 1994. 
37. M. S. Narasimhan and S. Ramanan, Existence of universal connections, Amer. J. Math. 83 (1961), 563-572. MR 0133772

38. S. P. Novikov and V. A. Rokhlin (eds.), Topology. II, Encyclopaedia of Mathematical Sciences, vol. 24, Springer-Verlag, Berlin, 2004, Homotopy and homology. Classical manifolds. MR 2054455

39. Daniel Quillen, Higher algebraic $K$-theory. I, Algebraic $K$-theory, I: Higher $K$-theories (Proc. Conf., Battelle Memorial Inst., Seattle, Wash., 1972), Lecture Notes in Mathematics, vol. 341, Springer, Berlin, 1973, pp. 85-147.

40. M. Sanders, Classifying spaces and Dirac operators coupled to instantons, Trans. Amer. Math. Soc. 347 (1995), no. 10, 4037-4072.

41. J. Santos, Topology of moduli spaces of rank stable instantons and holomorphic bundles, Ph.D. thesis, Stanford University, 2002.

42. 129-158.

43. Graeme Segal, Classifying spaces and spectral sequences, Inst. Hautes Études Sci. Publ. Math. (1968), no. 34, 105-112.

44. - Categories and cohomology theories., Topology 13 (1974), 293-312 (English).

45. Richard P. Stanley, Enumerative combinatorics. Volume 1, second ed., Cambridge Studies in Advanced Mathematics, vol. 49, Cambridge University Press, Cambridge, 2012.

46. Clifford Henry Taubes, Self-dual Yang-Mills connections on non-self-dual 4-manifolds, J. Differential Geom. 17 (1982), no. 1, 139-170.

47. _ Self-dual connections on 4-manifolds with indefinite intersection matrix, J. Differential Geom. 19 (1984), no. 2, 517-560.

48. ㄴ. The stable topology of self-dual moduli spaces, J. Differential Geom. 29 (1989), no. 1, 163-230.

49. Youliang Tian, The based SU(n)-instanton moduli spaces, Math. Ann. 298 (1994), no. 1, $117-139$.

50. Rainer M. Vogt, Convenient categories of topological spaces for homotopy theory, Arch. Math. (Basel) 22 (1971), 545-555.

Centro de Análise Matemática, Geometria e Sistemas Dinâmicos Departamento de Matemática, Instituto Superior Técnico, Universidade de Lisboa, Av. Rovisco Pais 1, 1049-001 Lisboa, Portugal

E-mail address: jsantos@math.tecnico.ulisboa.pt 